\title{
Search for the Standard Model
}

\section{Higgs Boson in the $W H \rightarrow \tau \nu b \bar{b}$ channel with the DØ Detector}

A thesis submitted to the University of Manchester for the degree of Doctor of

Philosophy in the Faculty of Engineering and Physical Sciences

2009

Philip Rich

Particle Physics Group

School of Physics and Astronomy 


\section{Contents}

$\begin{array}{ll}\text { Contents } & 1\end{array}$

Abstract $\quad 5$

$\begin{array}{lc}\text { Declaration } & 6\end{array}$

$\begin{array}{lr}\text { Acknowledgements } & 7\end{array}$

$\begin{array}{llr}\text { Chapter 1. Introduction } & 8\end{array}$

$\begin{array}{lll}\text { Chapter 2. Higgs Physics } & 10\end{array}$

$\begin{array}{lll}2.1 & \text { Theoretical Background } & 10\end{array}$

2.1.1 The Standard Model 10

2.1.2 Electroweak Symmetry Breaking 12

$\begin{array}{lll}2.1 .3 & \text { Higgs boson Phenomenology } & 14\end{array}$

$\begin{array}{lll}2.2 & \text { Higgs Boson Searches } & 16\end{array}$

$\begin{array}{lll}2.2 .1 & \text { Indirect Constraints } & 16\end{array}$

$\begin{array}{lll}2.2 .2 & \text { Direct Searches } & 17\end{array}$

$\begin{array}{lll}2.2 .3 & \text { The } W H \rightarrow \tau \nu b \bar{b} \text { Channel } & 19\end{array}$

$\begin{array}{lll}\text { Chapter 3. } & \text { Experimental Apparatus } & 23\end{array}$

3.1 The Tevatron 23 
3.2 The DØ Experiment 25

3.2.1 Detector Geometry 26

3.2.2 Central Tracking System 28

3.2.3 Central and Forward Preshower Detectors 31

3.2.4 Calorimeter 32

3.2.5 Inter Cryostat Detector 33

3.2.6 Muon System 34

$\begin{array}{lll}\text { 3.2.7 } & \text { Luminosity Monitor } & 36\end{array}$

$\begin{array}{lll}3.2 .8 & \text { Trigger System } & 38\end{array}$

Chapter 4. Analysis Tools 42

$\begin{array}{lll}4.1 & \text { Reconstruction } & 42\end{array}$

4.1.1 Lepton Identification 42

4.1.2 Jet Identification $\quad 49$

4.1.3 Missing Transverse Energy 50

4.1.4 b-jet Identification $\quad 51$

4.1.5 Multi-jet Triggers $\quad 55$

4.2 Simulation 58

4.2.1 Generation of Events $\quad 58$

$\begin{array}{lll}\text { 4.2.2 Correcting the MC Simulation } & 62\end{array}$

$\begin{array}{lll}\text { Chapter 5. } & \text { Modeling the } \tau \nu b \bar{b} \text { Final State } & 69\end{array}$

$\begin{array}{lll}5.1 & \text { Event Selection } & 69\end{array}$

$\begin{array}{lll}\text { 5.1.1 Data Samples } & 69\end{array}$

$\begin{array}{lll}5.1 .2 & \text { Object Selection } & 69\end{array}$

$\begin{array}{lll}\text { 5.1.3 Preselection Efficiency } & 74\end{array}$

$\begin{array}{lll}5.2 & \text { Background Modelling } & 74\end{array}$

5.2.1 Multi-jet Control Region 76 
$\begin{array}{lll}\text { 5.2.2 } & \text { MC Control Region } & 79\end{array}$

5.3 Modelling in the Analysis Sample 81

5.3.1 Preselection $\quad 83$

$\begin{array}{lll}5.3 .2 & \text { Selection } & 86\end{array}$

$\begin{array}{lll}\text { 5.3.3 One b-tag selection } & 89\end{array}$

$\begin{array}{lll}\text { 5.3.4 Two b-tag selection } & 92\end{array}$

Chapter 6. Search for Higgs boson in the $\tau \nu b \bar{b}$ Channel 98

6.1 Multivariate Analysis 98

$\begin{array}{lll}\text { 6.1.1 Decision Trees } & 98\end{array}$

$\begin{array}{lll}\text { 6.1.2 } & \text { BDT Input Distributions } & 100\end{array}$

$\begin{array}{lll}\text { 6.1.3 BDT Output Distributions } & 101\end{array}$

$\begin{array}{lll}6.2 & \text { Systematic Uncertainties } & 103\end{array}$

$\begin{array}{lll}\text { 6.2.1 Flat Systematics } & 103\end{array}$

$\begin{array}{lll}\text { 6.2.2 Shape Systematics } & 105\end{array}$

$\begin{array}{lll}6.3 & \text { Limit Setting } & 107\end{array}$

$\begin{array}{lll}\text { 6.3.1 Limit Setting Technique } & 107\end{array}$

$\begin{array}{lll}\text { 6.3.2 Fitting Uncertainties } & 109\end{array}$

$\begin{array}{lll}\text { 6.3.3 Results } & 111\end{array}$

$\begin{array}{lll}\text { Chapter 7. Discussion of Results } & 115\end{array}$

$\begin{array}{lll}7.1 & \text { Comparison with other channels } & 115\end{array}$

$\begin{array}{lll}7.2 & \text { Projections } & 117\end{array}$

$\begin{array}{lll}7.3 & \text { Comparison with the Previous Analysis } & 119\end{array}$

$\begin{array}{lll}\text { 7.3.1 Summary of } 1 \mathrm{fb}^{-1} \text { Result } & 120\end{array}$

7.3.2 Improvements over the $1 \mathrm{fb}^{-1}$ Result 123

7.3.3 Future Improvements to the $W H \rightarrow \tau \nu b \bar{b}$ Search 124 
Chapter 8. Conclusions

Appendix A. Kinematic Distributions

References 


\section{Abstract}

A search for the Standard Model Higgs boson is performed in $4.0 \mathrm{fb}^{-1}$ of $p \bar{p}$ collisions at $\sqrt{s}=1.96 \mathrm{TeV}$, collected with the $\mathrm{D} \varnothing$ detector at the Fermilab Tevatron. The final state considered is a pair of $b$-jets with large missing transverse energy and one hadronic tau decay as expected from the reaction $p \bar{p} \rightarrow W H \rightarrow \tau \nu b \bar{b}$. Boosted decision trees are used to discriminate the signal from the background. Good agreement is observed between data and expected backgrounds. For a Higgs boson mass of $115 \mathrm{GeV}$, a limit is set at 95\% C.L. on the cross-section times branching fraction of $(p \bar{p} \rightarrow(Z / W) H) \times(H \rightarrow b \bar{b})$ which is 14 times larger than the Standard Model value. 


\section{Declaration and Copyright}

No portion of the work referred to in this thesis has been submitted in support of an application for another degree or qualification of this or any other university or other institute of learning.

The author of this thesis (including any appendices and/or schedules to this thesis) own any copyright in it (the "Copyright") and he has given The University of Manchester the right to use such Copyright for any administrative, promotional, educational and/or teaching purposes.

Copies of this thesis, either in full or in extracts, may be made only in accordance with the regulations of the John Rylands University Library of Manchester. Details of these regulations may be obtained from the Librarian. This page must form part of any such copies made.

The ownership of any patents, designs, trade marks and any and all other intellectual property rigths except for the Copyright (the "Intellectual Property Rights") and any reproducations of copyright works, for example graphs and tables ("Reproductions"), which may be described in this thesis, may not be owned by the author and may be owned by third parties. Such Intellectual Property Rights and Reproductions cannot and must not be made available for use without the prior written permission of the owner(s) of the relevant Intellectual Property Rights and/or Reproductions.

Further information on the conditions which disclosure, publication and exploitation of this thesis, the Copyright and any Intellectual Property Rights and or Reproductions described in it may take place is available from the Head of School of Physics and Astronomy. 


\section{Acknowledgements}

I would first like to thank my supervisor Stefan Söldner-Rembold for all his assistance over the course of my $\mathrm{PhD}$. Especially in the past two years where despite holding high profile positions on the $\mathrm{D} \varnothing$ experiment, he has remained a constant source of guidance. My thanks also to all of the DØ Manchester group, especially Krisztian Peters for their help during my time at Fermilab. Thanks to Yvonne Peters for taking the time to read the whole thesis and her valuable advice. Special thanks to Mark Owen for his tireless ability to answer my questions, even when not working on DØ full time, as well as his friendship throughout my PhD.

Thanks also go to the many people I met during my time at Fermilab and to PPARC/STFC for funding my time spent at the lab and my $\mathrm{PhD}$. The results obtained in this $\mathrm{PhD}$ were only achievable via collaboration with the Higgs and Tau-ID groups at DØ. My thanks go to the conveners and members of those groups for all of their help.

My time in the Manchester HEP group has been very enjoyable due to the kind nature of the many characters within the group. Thanks to Fred Loebinger for being so approachable throughout all of my time in Manchester, and creating a vibrant atmosphere in the group. My vocabulary has no doubt been improved by our coffee time crosswords and lively banter.

I would like to thank my friends and family for offering all the support necessary, especially during the difficult times. Special thanks to my father for always having a belief in my abilities and being at the end of the phone when I needed it.

Finally, I would like to thank Laura for offering every kind of help throughout the last 4 years and much much more. 


\section{Chapter 1}

\section{Introduction}

The theory of Electroweak Symmetry Breaking explains how particles in the Standard Model (SM) obtain mass. This theory requires the presence of a fundamental scalar (spin-0) boson - the "Higgs" boson, whose existence needs to be confirmed by experiment.

Searching for a SM Higgs boson in the $W H \rightarrow \tau \nu b \bar{b}$ final state is difficult at a $p \bar{p}$ collider due to the hadronic nature of the collisions. However, as low mass Higgs searches at the Tevatron close in on SM sensitivity it is important to add as many channels to the combination as possible. This, along with upgraded analysis techniques and object identification, will give the Tevatron the best chance of observing a low mass Higgs boson before the end of running.

This thesis presents a search for a SM Higgs boson in the $\tau \nu b \bar{b}$ final state. This search uses data corresponding to an integrated luminosity of $4 \mathrm{fb}^{-1}$ accumulated by the $\mathrm{D} \varnothing$ detector between 2002 and 2009 .

The search for a low mass Higgs boson in the $\tau \nu b \bar{b}$ final state is presented in this thesis in the following way: Chapter 2 provides a theoretical background to Higgs boson physics focusing on phenomenology and current constraints on the Higgs boson mass from indirect sources and direct searches. Chapter 3 introduces 
the Tevatron accelerator and DØ experiment used in this analysis. Chapter 4 describes the tools used to reconstruct the $\tau \nu b \bar{b}$ final state and how simulation is used to understand how different processes appear in the detector. Chapter 5 details how the $\tau \nu b \bar{b}$ final state is selected in data. It additionally discusses the background modelling and how control regions are used to understand the data before probing regions sensitive to Higgs boson production. Chapter 6 details how Higgs boson production is searched for in the $\tau \nu b \bar{b}$ final state. The method of using a boosted decision tree to discriminate the Higgs boson signal from the background is presented. The treatment of systematic uncertainties in the analysis and the setting of limits on Higgs boson production cross-section are also described. Chapter 7 provides a discussion of the results of this thesis in the context of other searches in other channels and how much this channel adds to the overall Higgs search at $\mathrm{D} \varnothing$. A comparison to the previous Higgs boson search in the $\tau \nu b \bar{b}$ final state with $1 \mathrm{fb}^{-1}$ of $\mathrm{D} \varnothing$ data is shown. This analysis was also completed by the author, and was the first search of its kind, and the first published SM Higgs boson search in a final state with hadronic tau decays at a hadron collider. 


\section{Chapter 2}

\section{Higgs Physics}

This chapter provides an introduction to Higgs boson physics. First a motivation for the need for a Higgs boson in the Standard Model of particle physics (SM) and a description of Higgs boson phenomonology is given. Finally, a summary of the current status of searches for a Higgs boson is presented.

\subsection{Theoretical Background}

\subsubsection{The Standard Model}

As a particle physics theory the Standard Model has been incredibly successful in describing the phenomenology of particle interactions. Nearly all experimentally measured parameters of the theory have been found to be consistent with the predictions to a high degree of accuracy. This is indicated in Figure 2.1 [1] which shows the agreement between measurement and theory for a particular set of the free parameters in the SM. The pull indicates the number of standard deviations by which the measurement differs from the theory.

The SM is a quantum field theory and the symmetries of these fields dictate 


\begin{tabular}{|c|c|c|c|}
\hline & Measurement & Fit & $\mid \mathrm{O}^{\text {meas }}-\mathrm{O}^{\text {fit }} / / \sigma^{\text {meas }}$ \\
\hline$\overline{\Delta \alpha_{h a d}^{(5)}\left(m_{7}\right)}$ & $0.02758 \pm 0.00035$ & 0.02768 & 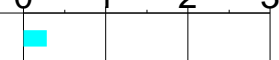 \\
\hline $\mathrm{m}_{\mathrm{z}}[\mathrm{GeV}]$ & $91.1875 \pm 0.0021$ & 91.1874 & \\
\hline$\Gamma_{Z}[\mathrm{GeV}]$ & $2.4952 \pm 0.0023$ & 2.4959 & 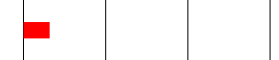 \\
\hline$\sigma_{\text {had }}^{0}[n b]$ & $41.540 \pm 0.037$ & 41.478 & \\
\hline $\mathrm{R}_{1}$ & $20.767 \pm 0.025$ & 20.742 & 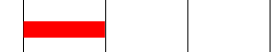 \\
\hline$A_{f b}^{0,1}$ & $0.01714 \pm 0.00095$ & 0.01645 & \\
\hline$A_{1}\left(P_{\tau}\right)$ & $0.1465 \pm 0.0032$ & 0.1481 & 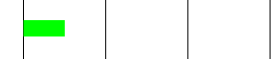 \\
\hline $\mathrm{R}_{\mathrm{b}}$ & $0.21629 \pm 0.00066$ & 0.21579 & r \\
\hline $\mathrm{R}_{\mathrm{c}}$ & $0.1721 \pm 0.0030$ & 0.1723 & 1 \\
\hline$A_{\mathrm{fb}}^{\mathrm{C}, \mathrm{b}}$ & $0.0992 \pm 0.0016$ & 0.1038 & \\
\hline$A_{\mathrm{fb}}^{0, \mathrm{c}}$ & $0.0707 \pm 0.0035$ & 0.0742 & \\
\hline$A_{b}$ & $0.923 \pm 0.020$ & 0.935 & 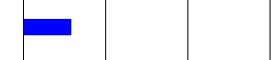 \\
\hline$A_{c}$ & $0.670 \pm 0.027$ & 0.668 & 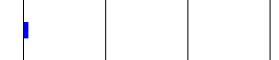 \\
\hline$A_{l}(S L D)$ & $0.1513 \pm 0.0021$ & 0.1481 & \\
\hline $\sin ^{2} \theta_{\text {eff }}^{\text {lept }}\left(Q_{\mathrm{fb}}\right)$ & $0.2324 \pm 0.0012$ & 0.2314 & \\
\hline $\mathrm{m}_{\mathrm{W}}[\mathrm{GeV}]$ & $80.399 \pm 0.023$ & 80.379 & 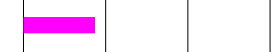 \\
\hline$\Gamma_{\mathrm{W}}[\mathrm{GeV}]$ & $2.098 \pm 0.048$ & 2.092 & P \\
\hline$m_{t}[\mathrm{GeV}]$ & $173.1 \pm 1.3$ & 173.2 & l \\
\hline August 2009 & & & 0 \\
\hline
\end{tabular}

Figure 2.1: Agreement between the fit to the theory parameters and measurement (pull) for a set of the free parameters of the SM [1].

the interactions between the spin- $\frac{1}{2}$ fermions (matter) mediated by spin-1 bosons. Fermions are classified by their interactions into quarks and leptons. Quarks interact through the strong force which is mediated by the massless gluon and is described by the theory of quantum chromodynamics (QCD). Quarks along with leptons interact via the weak force which is mediated by the massive $W^{ \pm}$ and $Z$ bosons. Electromagnetic (EM) interactions between charged particles are mediated by the massless photon. The EM and weak forces are unified in the 
electroweak interaction (see Section 2.1.2).

The fermions are arranged into three generations. The mass of the fermions increases by generation, but this phenomenon is not explained by the SM. Each generation contains a pair of quarks and a pair of leptons forming a left-handed weak isospin doublet and a right-handed singlet. For each of the fermions in each generation there exists an anti-particle whose mass and spin are identical but has opposite charges (flavour, colour, electric). The two quarks in each generation have electric charges of $+\frac{2}{3}$ for up-type quarks and $-\frac{1}{3}$ for down-type quarks. The two leptons in each generation have electric charge -1 and 0 . This generation structure of the SM fermions and their associated properties is summarised in Table 2.1.

\begin{tabular}{|c|c|c|c|c|c|c|}
\hline Fermion Type & \multicolumn{2}{|c|}{ 1st Generation } & \multicolumn{2}{|c|}{ 2nd Generation } & \multicolumn{2}{|c|}{ 3rd Generation } \\
\hline Quarks & $\mathbf{U}$ & $\mathbf{d}$ & $\mathbf{C}$ & $\mathbf{S}$ & $\mathbf{t}$ & $\mathbf{b}$ \\
Leptons & $\begin{array}{c}\text { Up } \\
\mathrm{m}=1.5-3.3\end{array}$ & $\begin{array}{c}\text { Down } \\
\text { Charge }-\frac{1}{3} \\
\mathrm{~m}=3.5-6.0\end{array}$ & $\begin{array}{c}\text { Charm } \\
\text { Charge }+\frac{2}{3} \\
\mathrm{~m}=1270_{-110}^{+30}\end{array}$ & $\begin{array}{c}\text { Strange } \\
\text { Charge }-\frac{1}{3} \\
\mathrm{~m}=104_{-34}^{+26}\end{array}$ & $\begin{array}{c}\text { Top } \\
\text { Charge }+\frac{2}{3} \\
\mathrm{~m}=(1.712 \pm 0.021) \times 10^{5}\end{array}$ & $\begin{array}{c}\text { Bottom } \\
\text { Charge }-\frac{1}{3} \\
\mathrm{~m}=4200_{-70}^{+770}\end{array}$ \\
\hline $\begin{array}{c}\text { Electron } \\
\text { Charge }-1 \\
\mathrm{~m}=0.511\end{array}$ & $\begin{array}{c}\text { Electron Neutrino } \\
\text { Charge } 0 \\
\mathrm{~m}=<2 \times 10^{-6}\end{array}$ & $\begin{array}{c}\text { Muon } \\
\text { Charge }-1 \\
\mathrm{~m}=105.7\end{array}$ & $\begin{array}{c}\text { Muon Neutrino } \\
\text { Charge } 0 \\
\mathrm{~m}=<0.19\end{array}$ & $\begin{array}{c}\text { Tau } \\
\text { Charge }-1 \\
\mathrm{~m}=1777\end{array}$ & $\begin{array}{c}\text { Tau Neutrino } \\
\text { Charge } 0 \\
\mathrm{~m}=<18.2\end{array}$ \\
\hline
\end{tabular}

Table 2.1: Properties of the fermions in the SM [2], $\mathrm{m}$ indicates the mass of the fermion in $\mathrm{MeV}$.

\subsubsection{Electroweak Symmetry Breaking}

Having massless bosons in the theories of QED and QCD is consistent with observation. The $W$ and $Z$ bosons, however, have been observed to have masses of $80.398 \pm 0.025 \mathrm{GeV}$ and $91.1876 \pm 0.0021 \mathrm{GeV}$, respectively [2]. To include this effect in the theory would require the addition of mass terms in the Lagrangian, breaking the gauge symmetry of the theory. The theory would then be nonrenormalisable and contain divergences leading to unphysical results. It will also 
destroy the symmetry that gave rise to the force carriers in the theory.

The concept of spontaneous symmetry breaking (SSB) allows the theory to remain invariant under symmetry transformations, but the ground state of the potential to not be invariant. This is illustrated in Figure 2.2 [3] which shows the so called "Mexican hat potential". The potential $\phi=\left(\phi_{R e}, \phi_{I m}\right)$ is symmetric under rotations in the $\phi_{R e} \times \phi_{I m}$ plane, however, the rotational symmetry will be broken when the particle moves to the ground state.

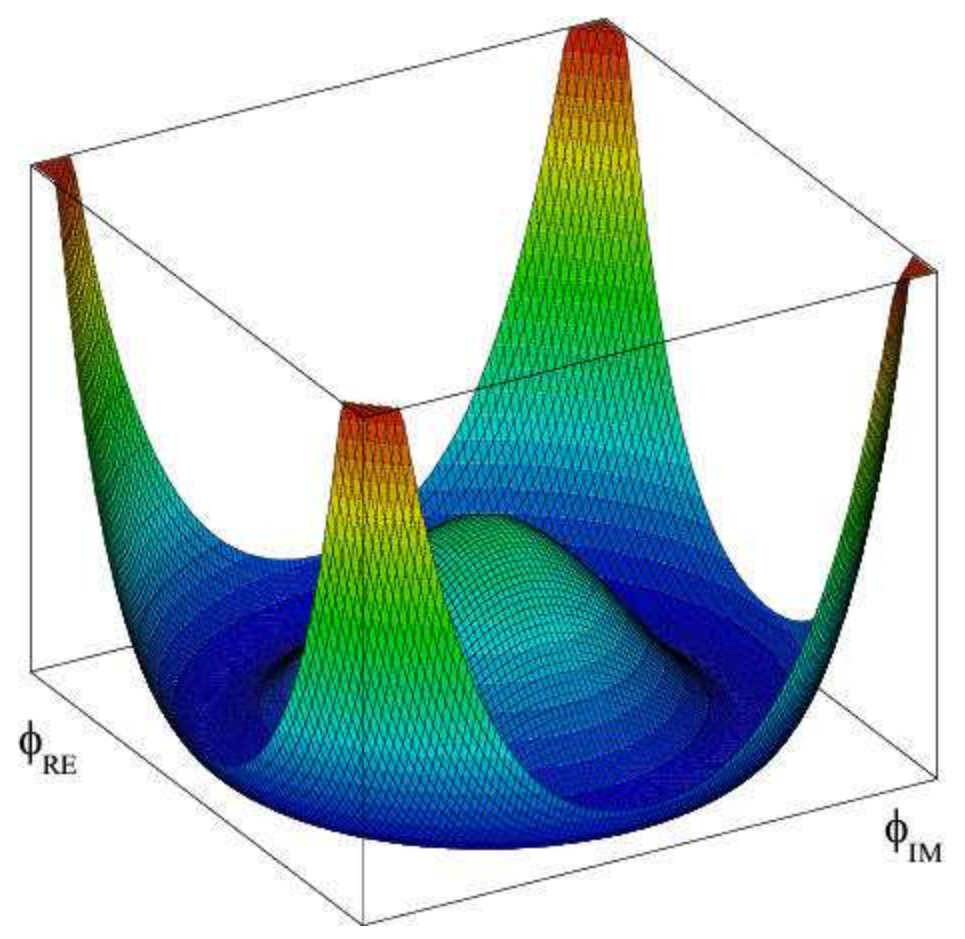

Figure 2.2: Diagram of the spontaneous symmetry breaking "Mexican hat" potential for a complex potential $\phi=\left(\phi_{R e}, \phi_{\text {Im }}\right)[3]$.

A consequence of this symmetry breaking is the requirement of a new fundamental spin-0 boson - the "Higgs Boson", the only fundamental scalar in the SM. The mass of the Higgs boson is not predicted by the theory. Several SM variables are sensitive to the mass of the Higgs boson via loop corrections (see Section 2.2.1). 


\subsubsection{Higgs boson Phenomenology}

The Higgs boson couples most strongly to the heaviest particles and this is the dominant feature of any Higgs boson search strategy. This section describes Higgs boson phenomenology before the latest results on Higgs boson searches are presented.

Figure 2.3 [4] shows the theoretical production cross-sections for a SM Higgs boson at the Tevatron as a function of the mass of the Higgs boson $m_{h}$.

SM Higgs production

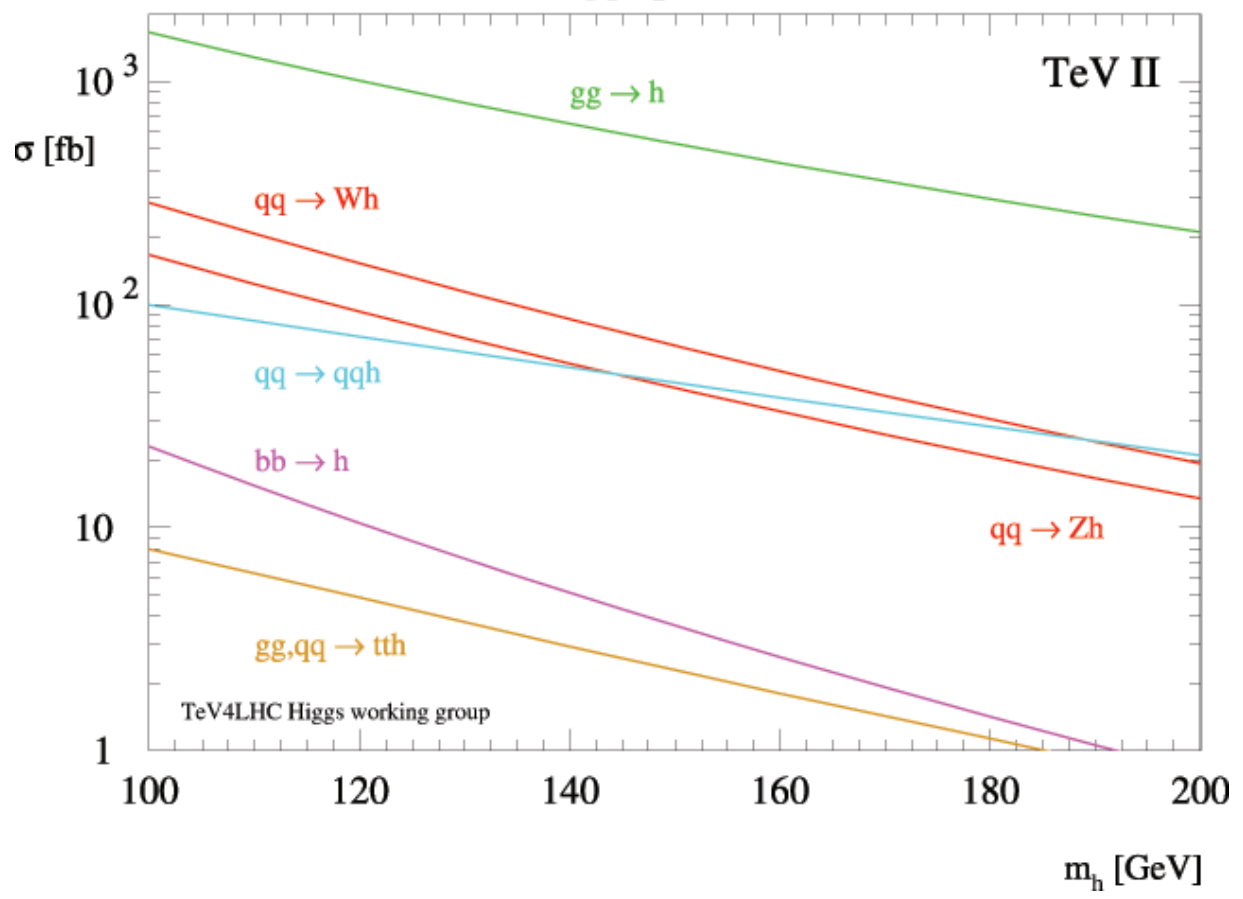

Figure 2.3: Production cross-sections for a SM Higgs boson at the Tevatron as a function of Higgs boson mass $m_{H}[4]$.

The cross-sections decrease as the mass of the Higgs boson increases, due to the extra energy required to create a heavier Higgs boson. The dominant production mechanism for a SM Higgs boson at the Tevatron is gluon-gluon fusion $(g g \rightarrow H)$. Associated production $(q \bar{q} \rightarrow W H / Z H)$ is where the Higgs boson is radiated by an energetic $W$ or $Z$ boson produced via quark interactions, 
and has a cross-section around ten times less than gluon-gluon fusion. These two production mechanisms dominate searches for a SM Higgs boson at the Tevatron. Feynman diagrams showing these two production mechanisms for a Higgs boson and its subsequent decays are shown in Figure 2.4.

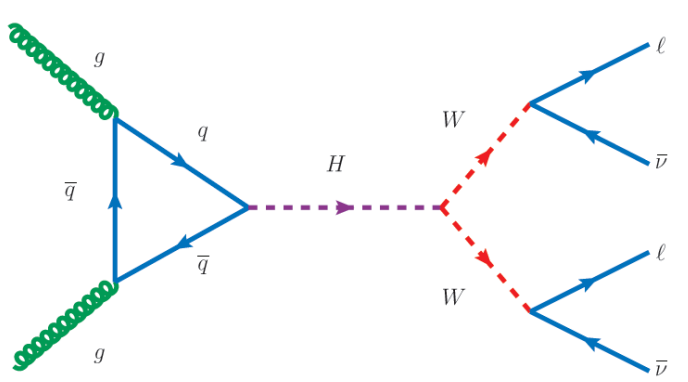

(a) gluon-gluon fusion

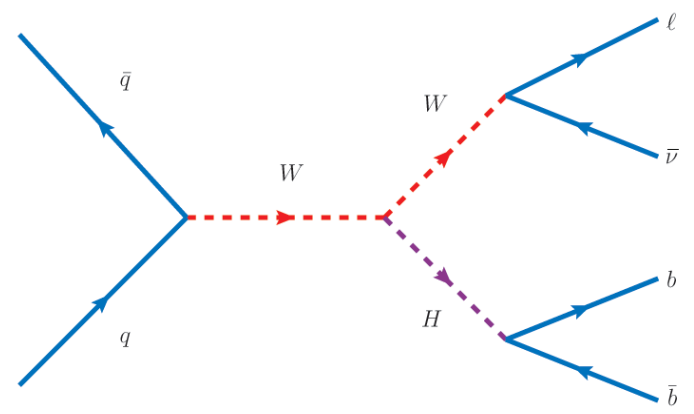

(b) associated production

Figure 2.4: Feynman diagrams showing Higgs boson production via (a) gluon-gluon fusion and (b) association with a vector boson.

For a Higgs boson whose mass is of order the electroweak scale, the decay will occur very quickly inside the detector and so the reconstruction of the Higgs boson must be done via its decay products. The theoretical branching ratios for a SM Higgs boson are shown in Figure 2.5 [5].

For a low mass Higgs boson $\left(m_{H}<140 \mathrm{GeV}\right)$ the dominant decay mode is to b-quarks $(H \rightarrow b \bar{b})$. Searching for a $b \bar{b}$ signature in the hadronic environment of the Tevatron is extremely difficult as the signature is similar to the dominant multi-jet background. For a low mass Higgs boson associated production is more sensitive as the leptonic decay products of the $W$ or $Z$ boson are used to tag the event and reject a large amount of the multi-jet background.

For a higher Higgs boson mass the decay into $W W$ pairs is most sensitive. This channel benefits from the gluon-gluon fusion production mechanism as the leptonic decay products of the $W$ bosons are sufficient to reduce the multi-jet background to acceptable levels. 


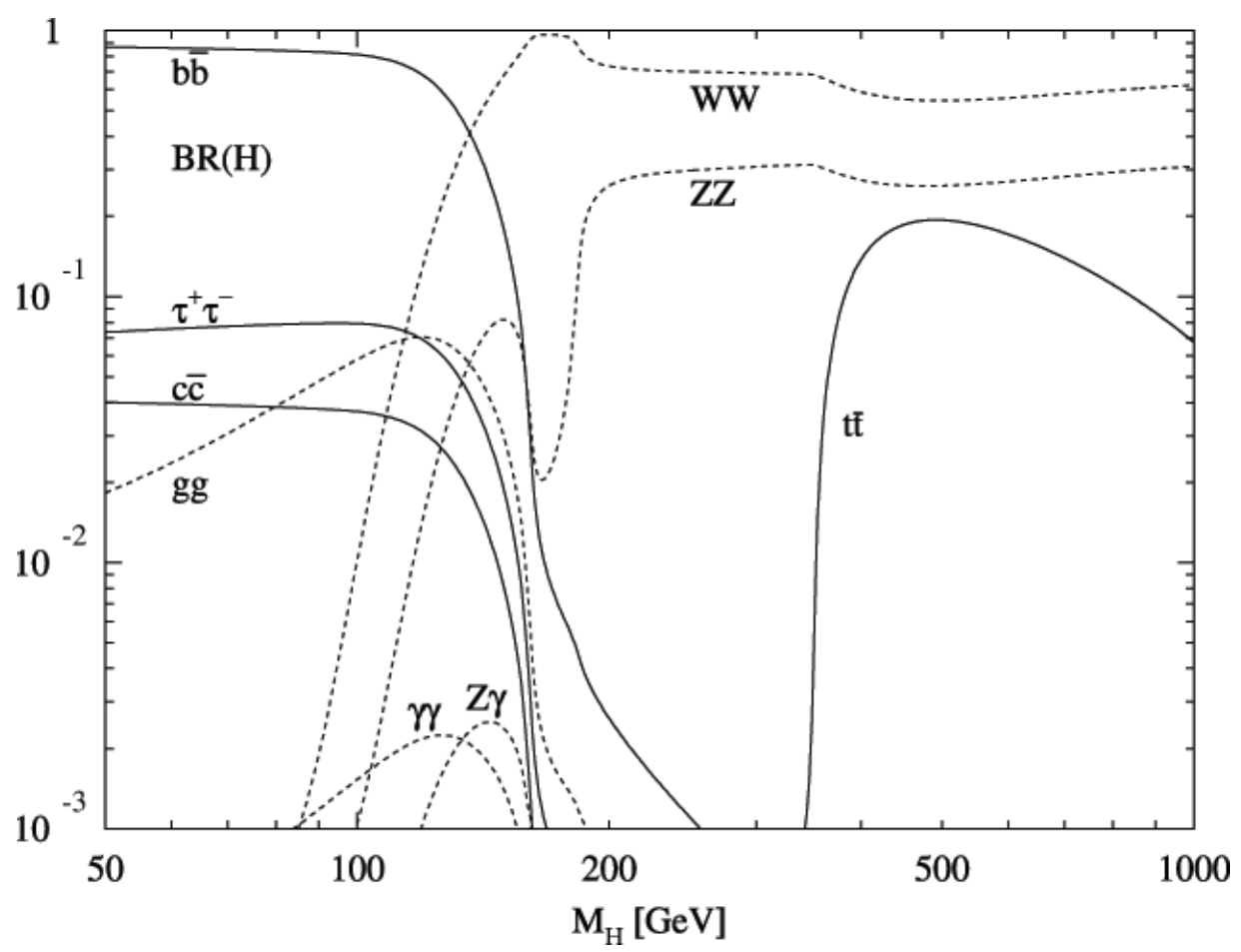

Figure 2.5: Branching ratios for a SM Higgs boson as a function of Higgs boson mass $m_{H}$ [5].

\subsection{Higgs Boson Searches}

This section describes the status of Higgs boson searches. Indirect constraints come from precision measurements of parameters sensitive to the mass of the Higgs boson. Direct searches are based upon the predicted coupling of the Higgs boson to other SM particles from theory.

\subsubsection{Indirect Constraints}

Radiative loop effects have a logarithmic dependence on the mass of the Higgs boson [6]. By precision measurements of the $W$ boson and top quark masses confidence level limits can be placed on the mass of the Higgs boson. Figure 2.6 [1] shows the measured $W$ mass against the measured top quark mass with a one standard deviation confidence level indicating the constraints on the mass of the 
Higgs boson. The linear bands on the plot indicate the Higgs mass for a given $W$ boson and top quark mass. The LEP2 and Tevatron data combined contour favours a light Higgs boson mass.

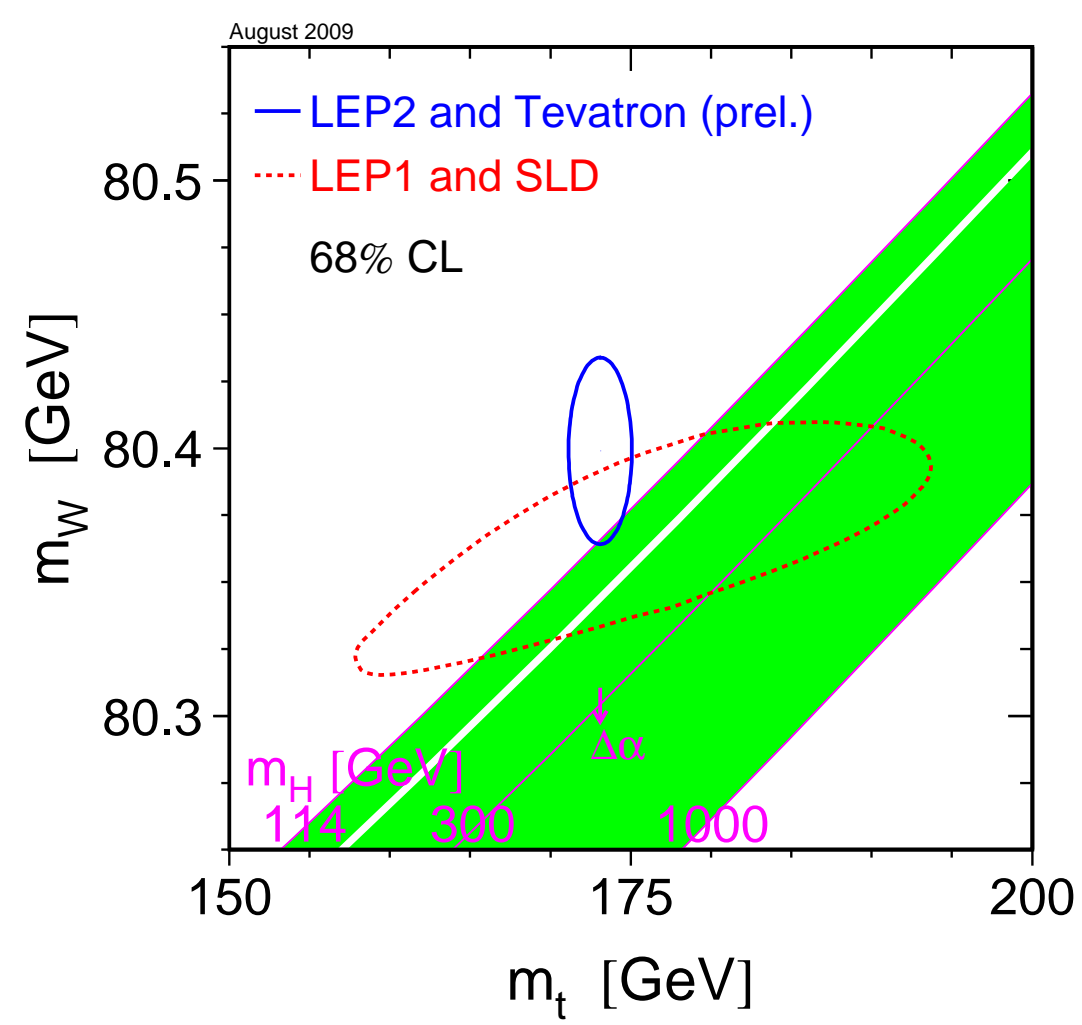

Figure 2.6: Mass of the $\mathrm{W}$ boson vs mass of the top quark with $68 \%$ confidence level contour indicating the contraint on the mass of the Higgs boson. The dashed band indicates the constraint from LEP1 and SLD and the solid band indicates the constraint from LEP2 and Tevatron results [1]. The white band at low Higgs boson mass indicates the Tevatron exclusion of a Higgs boson masses between $160 \mathrm{GeV}$ to $170 \mathrm{GeV} . \Delta \alpha$ indicates the direction that the green bands shift under a systematic shift of the fit parameters.

\subsubsection{Direct Searches}

The direct searches based upon the known production mechanisms and couplings of the Higgs boson (for a given mass) to other SM particles are complimentary to the indirect constraints. A lower limit on the mass of the Higgs boson was 
set at the LEP2 experiments which searched for production of a Higgs boson in association with a $Z$ boson in $e^{+} e^{-}$collisions. The results yielded a limit of $m_{H}>$ 114.4 GeV [7] at 95\% confidence level, just below the kinematic threshold of the experiments due to a slight excess in the final sample.

Higgs boson searches at the Tevatron benefit from a greater centre-of-mass energy. At a $p \bar{p}$ collider the hadronic background is much larger and so the backgrounds and search strategies differ from those used at the LEP experiments. The results from searches across multiple channels from the D $\varnothing$ and CDF experiments are combined into a Tevatron limit on Higgs boson production. Figure 2.7 [8] shows the Tevatron combined limits as a function of the mass of the Higgs boson. A Higgs boson of mass between 160 - $170 \mathrm{GeV}$ is excluded at $95 \%$ confidence level. This is indicated by the observed limit divided by the SM predicted crosssection passing below unity on the plot. This is the first SM Higgs boson mass exclusion since the LEP result. This figure also illustrates the sensitivity of the Tevatron to a low mass Higgs boson, where the combination of many low mass channels enables the exclusion of cross-sections a factor of two greater than those predicted by the SM. A summary of SM Higgs boson searches at D $\varnothing$, including this Tevatron combination was made as conference proceedings for the PANIC 2008 conference after a talk was given by the author [9]. This Tevatron combination includes the $W H \rightarrow \tau \nu b \bar{b}$ analysis using $1 \mathrm{fb}^{-1}$ of $\mathrm{D} \varnothing$ data performed by the author, which is published in [10] and further discussed in Section 7.

Figure 2.8 [1] summarises the current Higgs boson searches, showing the EW fit and the LEP and Tevatron exclusions. The fit prefers a Higgs boson mass $m_{H}$ $=87_{-26}^{+35} \mathrm{GeV}$ at $68 \%$ confidence and requires $m_{H}<157 \mathrm{GeV}$ at $95 \%$ confidence. When the LEP2 exclusion is added into the fit the upper limit on the Higgs boson mass becomes $m_{H}<186 \mathrm{GeV}$ at $95 \%$ confidence [1]. 


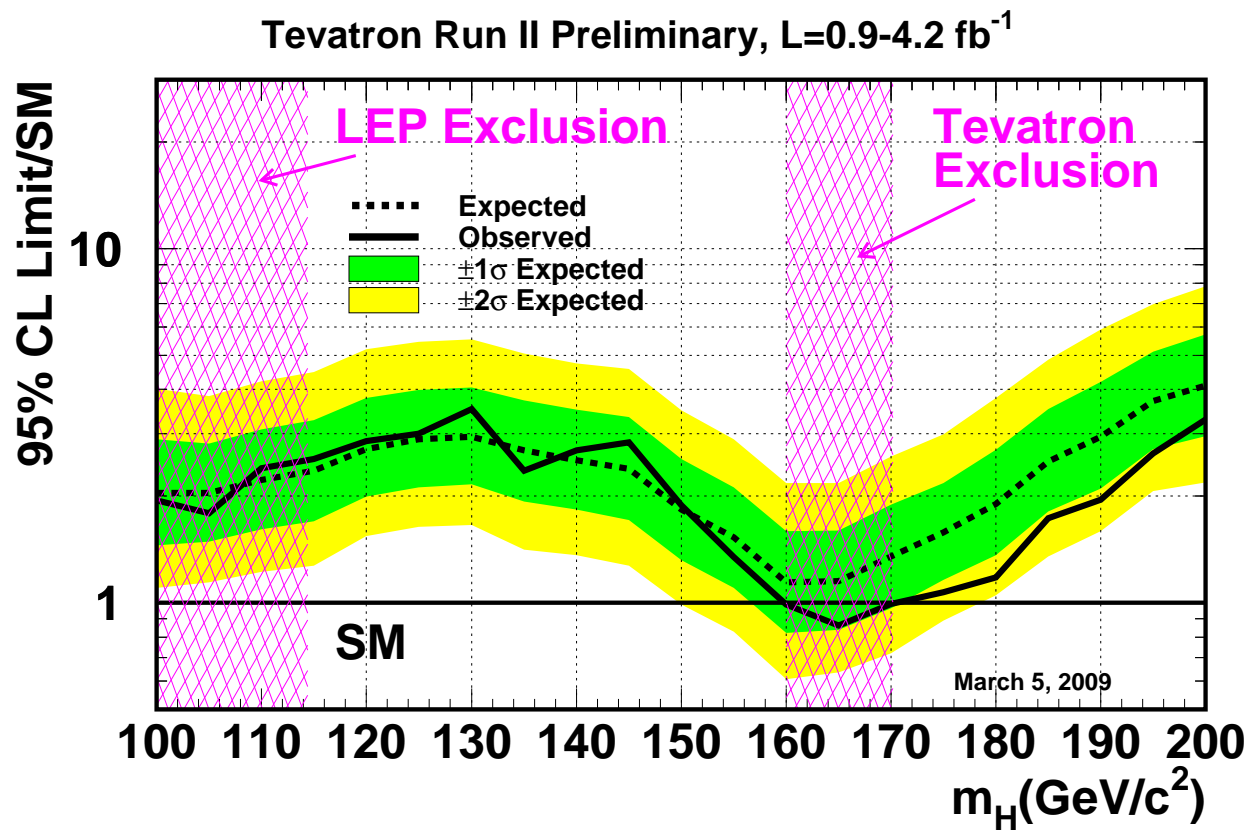

Figure 2.7: 95\% confidence limits on the SM Higgs boson production cross-section relative to the SM cross-section at the Tevatron as a function of the Higgs boson mass $m_{H}$ [8].

\subsubsection{The $W H \rightarrow \tau \nu b \bar{b}$ Channel}

As the Tevatron experiments become more sensitive to a low mass Higgs boson it is important to extract sensitivity from every possible channel. The $W H \rightarrow \tau \nu b \bar{b}$ channel is difficult to reconstruct due to the complexity of the particles in the final state. A brief summary of the phenomenology of the final state particles in this channel is given below. The neutrino is not discussed separately here as it escapes detection and is inferred by the existance of "Missing transverse energy" in the detector (see Section 4.1.3). 


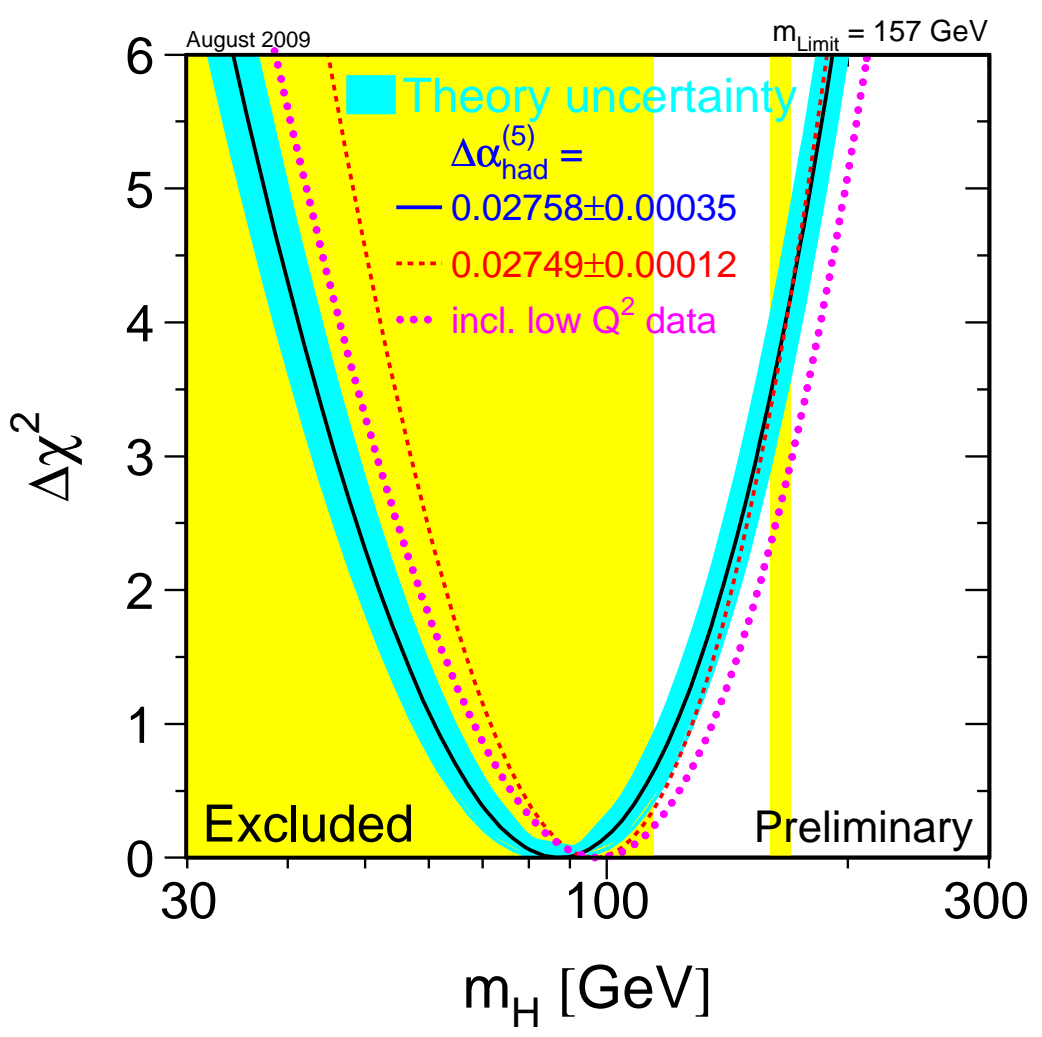

Figure 2.8: Fit for the SM Higgs mass using the parameters of the SM, the so called "blue band plot" [1]. The blue band indicates the theoretical uncertainty and the other contours indicate the range of values for the fit when the quark sector contribution (without top), $\Delta \alpha_{\text {had }}^{(5)}$ to the coupling is varied as indicated.

\section{The b-quark}

As a 3rd generation particle the b-quark has a relatively large mass $\left(m_{b}=\right.$ $\sim 4.20 \mathrm{GeV}[2])$ and many potential decay paths. After a b-quark is produced it rapidly hadronises into a B-hadron (mostly into B-Mesons - a bound quarkanti-quark state) whose lifetimes are of order $10^{-12}$ s. Table $2.2[2]$ shows the characteristics of B-meson bound states.

These lifetimes allow the B-mesons to travel around $500 \mu m$ (at the typical momentum ranges observed at the Tevatron) before they decay. When they do 


\begin{tabular}{|c|c|c|c|}
\hline B-Meson type & Constituent quarks & Mass $(\mathrm{MeV})$ & Lifetime $(\mathrm{ps})$ \\
\hline$B^{ \pm}$ & $u b / \bar{u} b$ & $5,279.15 \pm 0.31$ & $1.638 \pm 0.011$ \\
\hline$B^{0} / B^{0}$ & $d \bar{d} / \bar{d} b$ & $5,279.53 \pm 0.33$ & $1.530 \pm 0.009$ \\
\hline$B_{s}^{0} / B_{s}^{0}$ & $s b / \bar{s} b$ & $5,366.3 \pm 0.6$ & $1.470_{-0.026}^{+0.027}$ \\
\hline$B_{c}^{ \pm}$ & $c b / \bar{c} b$ & $6,276 \pm 4$ & $0.46 \pm 0.07$ \\
\hline
\end{tabular}

Table 2.2: B-Meson characteristics [2].

decay, the b-quarks themselves decay hadronically around $70 \%$ of the time and in semi-leptonic decay modes around $30 \%$ of the time. Some hadronic modes are suppressed by the CKM matrix, a full description of which is available in [11]. These decays and other rarer decay modes for the b-quark are shown in Figure 2.9 [12].

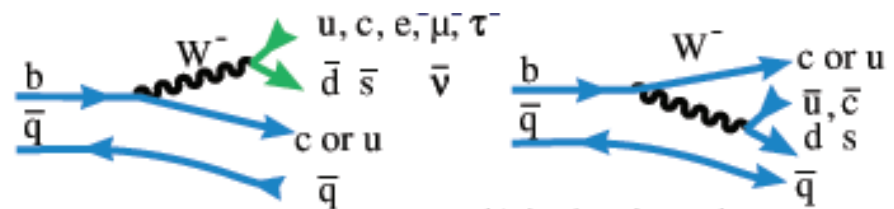

a) simple spectator

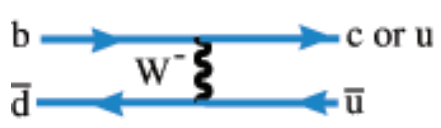

d) $\mathrm{W}$ exchange b) hadronic: color suppressed

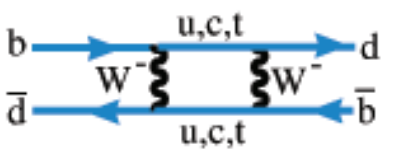

e) box: mixing

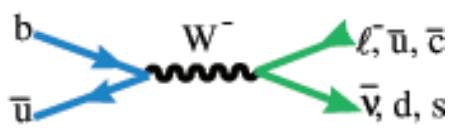

c) annihilation

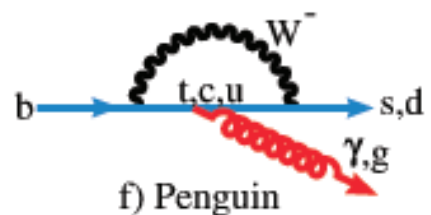

f) Penguin

Figure 2.9: Decay modes of b-quarks [12].

\section{The tau-lepton}

Similar to the b-quark, the tau-lepton is a heavy 3rd generation particle $\left(m_{\tau}=\right.$ $1776.84 \pm 0.17 \mathrm{MeV}[2])$ and has many different decay modes. The average lifetime of the tau lepton is $(290.6 \pm 1.0) \times 10^{-15} \mathrm{~s}$ and so at typical momentum ranges observed at the Tevatron the tau decays in around $87 \mu \mathrm{m}$. The tau-lepton is therefore only detectable by its decay products. A summary of the decay modes is shown in Table $2.3[2]$. 


\begin{tabular}{|c|c|}
\hline$\tau$ decay mode & Branching Ratio \\
\hline$\pi^{-} \pi^{0} \pi^{0} \nu_{\tau}$ & 0.0925 \\
\hline$\pi^{-} \pi^{+} \pi^{-} \nu_{\tau}$ & 0.0899 \\
\hline$\pi^{-} \pi^{0} \nu_{\tau}$ & 0.255 \\
\hline$\pi^{-} \nu_{\tau}$ & 0.109 \\
\hline$\mu^{-} \bar{\nu}_{\mu} \nu_{\tau}$ & 0.174 \\
\hline$e^{-} \overline{\nu_{e}} \nu_{\tau}$ & 0.178 \\
\hline 25 Other Modes & 0.102 \\
\hline
\end{tabular}

Table 2.3: Tau lepton decay processes, the final row indicates the sum of all remaining modes $[2]$.

Each of the tau lepton decay modes are mediated by the weak force. Feynman diagrams for a leptonic and hadronic decay process are shown in Figure 2.10.

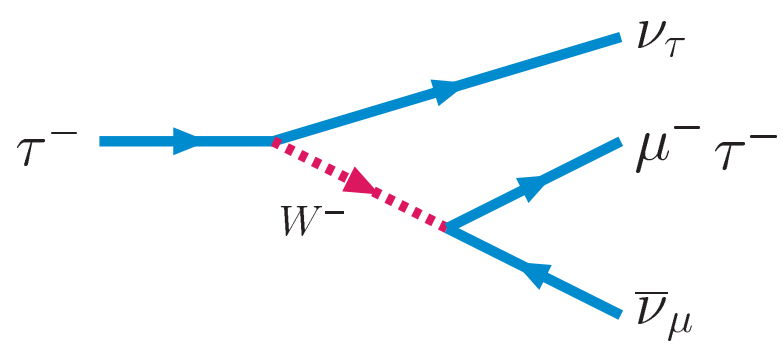

(a) Leptonic tau decay

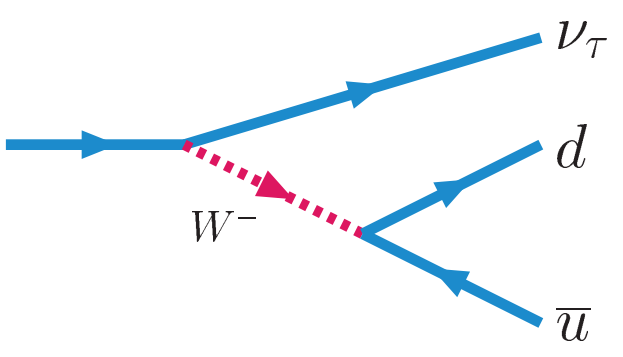

(b) Hadronic tau decay

Figure 2.10: Decay modes of the tau lepton showing (a) leptonic tau decay into a muon and (b) hadronic tau decay into a pion.

The dominant hadronic decay modes of the tau are "single-pronged" arising from only one charged pion in the final state. Single-pronged decays account for $46 \%$ of all tau decays and $70 \%$ of hadronic tau decays [2]. 


\section{Chapter 3}

\section{Experimental Apparatus}

Creating the heaviest fundamental particles requires high energy collisions. In particle accelerators strong electric fields are used to accelerate charged particles to a speed close to the speed of light. This energy is then released in collisions with either a fixed target or another accelerated beam in the opposite direction. Located at the Fermi National Accelerator Laboratory near Chicago, USA, the Tevatron collides protons and antiprotons at a centre of mass energy of $1.96 \mathrm{TeV}$ at two collision points around a four mile ring. Two experiments, CDF and DØ, surround these collision points. Both multi-purpose cylindrical detectors are aimed at a wide range of physics analyses. An overview of the Tevatron accelerator and the $\mathrm{D} \varnothing$ experiment is given below.

\subsection{The Tevatron}

Accelerating protons and antiprotons to such high energies is a gradual process. The Tevatron has a sequence of accelerators that increase in size and energy range. Additionally, storage rings are required to hold the protons and antiprotons before acceleration. A schematic of the Tevatron apparatus is shown in Figure 3.1. 
Proton production begins inside the Cockroft-Walton accelerator which passes Hydrogen through an electric field leaving $\mathrm{H}^{-}$ions, and accelerates them to $750 \mathrm{keV}$. The negatively charged ions are then passed on to a linear accelerator (LINAC) and are accelerated to $400 \mathrm{MeV}$ using radio frequency (RF) fields. Electrons are then stripped from the ions by passing the beam through a graphite foil, such that only a proton beam remains. Further acceleration of this proton beam occurs in the booster where the protons are constrained to a circular path using dipole magnets and accelerated using RF cavities to $8 \mathrm{GeV}$. Then the protons are passed into the Main Injector and accelerated to $150 \mathrm{GeV}$ before the beam is passed into the Tevatron for final acceleration to $980 \mathrm{GeV}$.

Antiproton production occurs via the Main Injector, where a proton beam is accelerated to an energy of $120 \mathrm{GeV}$ and then collided on a copper-nickel target, producing one antiproton for every $10^{5}$ colliding protons. The antiprotons then undergo the process of stochastic cooling, where electric charge feedback loops reduce the tendency of particles to move away from each other [13], and are accelerated to $8 \mathrm{GeV}$ in the debuncher. The antiprotons are moved to the recycler via the accumulator storage ring. The recycler cools the antiproton beam and once enough antiprotons have been collected they are passed into the Main Injector and accelerated to $150 \mathrm{GeV}$. Like the protons they are then injected into the Tevatron ring for acceleration to $980 \mathrm{GeV}$.

The protons and antiprotons are injected into the Tevatron in a series of bunches. There are 36 bunches in total, separated by 396 ns. These bunches are grouped into 3 superbunches separated by $2 \mu \mathrm{s}$. The beams are focused with magnets and collided at two interaction points surrounded by CDF and DØ. Typical initial instantaneous luminosities at the Tevatron lie in the range $2-3 \times 10^{32} \mathrm{~cm}^{-2} \mathrm{~s}^{-1}$ decaying over the course of the store which usually lasts $\sim 20$ hours. The length of the store is optimised to give the highest integrated 
luminosity based upon current instantaneous luminosity and stack of antiprotons available. A more detailed overview of the Tevatron acceleration and running procedure can be found in [14].

\section{FERMILAB'S ACCELERATOR CHAIN}

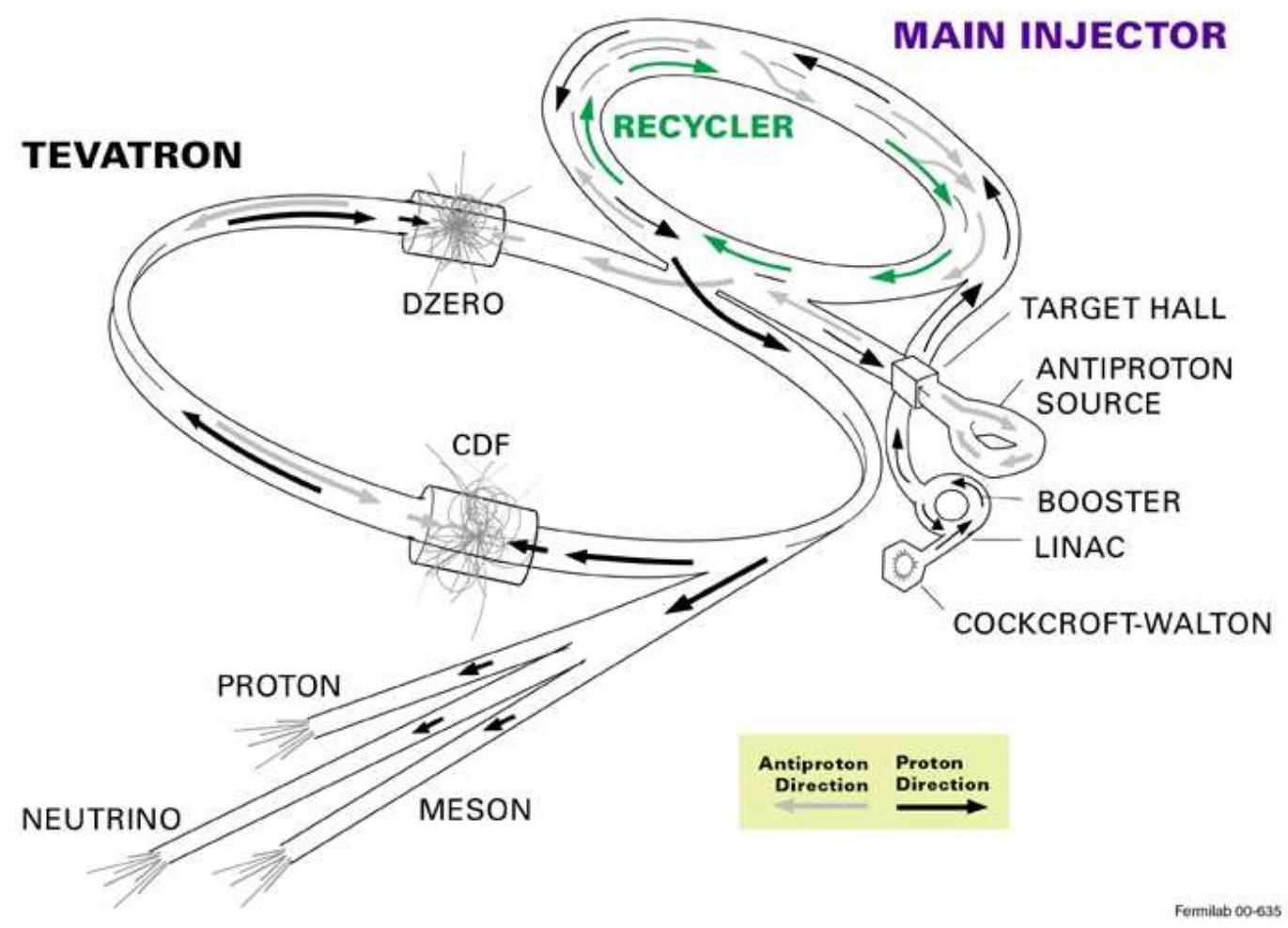

Figure 3.1: Schematic of the Tevatron accelerating complex.

\subsection{The DØ Experiment}

The DØ Experiment is a cylindrical multi-purpose detector. A schematic of the detector is shown in Figure 3.2 [15].

Nearest to the beam line and collision point are the tracking detectors - the Silicon Microstrip Tracker (SMT) and the Central Fibre Tracker (CFT). Outside of the tracker are the Electro-Magnetic (EM) and Hadronic Calorimeters, followed 


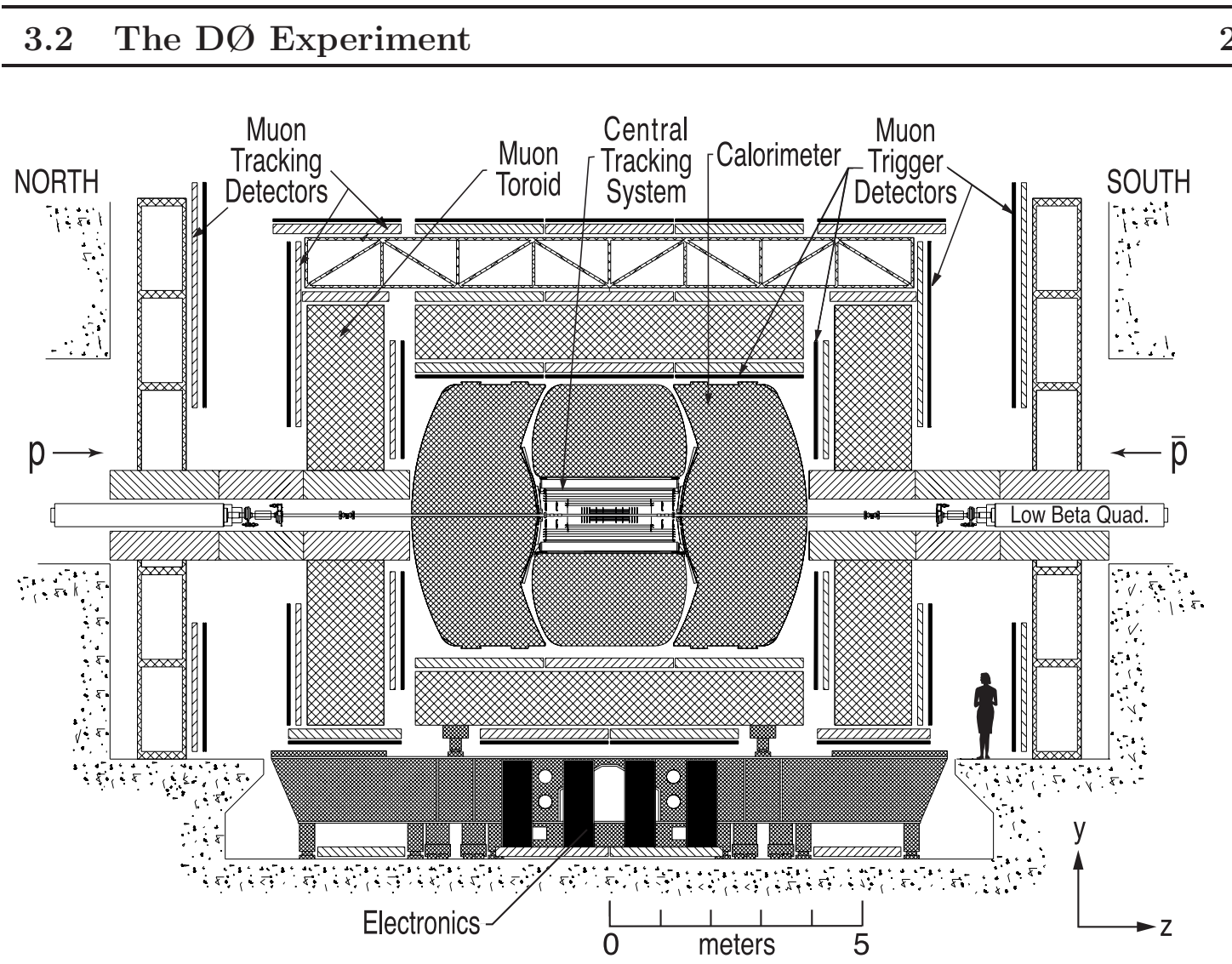

Figure 3.2: Diagram of the RunII D $\varnothing$ detector [15].

by the Muon detector subsystem. A three level trigger system with hardware and software components selects events to be stored in real time. Luminosity monitors measure the integrated and instantaneous luminosity delivered to the experiment from the Tevatron. Each of these systems and subsystems is described briefly in the next sections as well as a reference to the coordinate system used in the experiment.

\subsubsection{Detector Geometry}

To understand the nomenclature used in the $\mathrm{D} \varnothing$ detector the following describes the right handed coordinate system used by the experiment. The three dimensional coordinates are defined as:

- $+x$ : Radially outwards from the Tevatron ring, 
- $+y$ : Upwards,

- $+z$ : In the proton beam direction.

The $\mathrm{D} \varnothing$ detector is approximately cylindrical and this motivates the use of a $(\theta, \phi, z)$ coordinate system. Here $\theta$ is the angle from the $+z$ axis and $\phi$ is defined as being equal to zero at $+x$ and equal to $\pi / 2$ at $+y$. One problem with such a definition is that $\theta$ is not a Lorentz invariant quantity, and so another variable, the rapidity $y$, is defined as:

$$
y=\frac{1}{2} \ln \left(\frac{E+p_{z}}{E-p_{z}}\right)
$$

where $p_{z}$ is the momentum component along the $z$ axis and $E$ is the total energy. The pseudorapidity $\eta$ is defined as:

$$
\eta=-\ln \left(\tan \frac{\theta}{2}\right) .
$$

and corresponds to the rapidity in the limit $m / E \rightarrow 0$ and has the advantage that only the angle $\theta$ is required to be known.

It is also useful to consider $\eta$ in two different frames. Firstly, "physics $\eta$ ", $\eta_{p}$ which is defined with respect to the primary interaction vertex. Secondly, "detector $\eta ", \eta_{d}$ which is defined with respect to the centre of the detector at the origin of the coordinate system. The variable $\Delta R$ is used to determine the separation of reconstructed objects in the detector and is defined as:

$$
\Delta R=\sqrt{(\Delta \phi)^{2}+(\Delta \eta)^{2}}
$$

Since the difference between two pseudo-rapidities is Lorentz-invariant under longitudinal boosts, $\Delta R$ is also Lorentz-invariant under the same boosts. 
In most high energy collisions only a small momentum exchange occurs. However, most of the interesting physics (certainly for the scope of this thesis) occurs from hard scattering where particles leave the interaction point with a large momentum component transverse to the beam pipe. The transverse momentum is defined as:

$$
p_{T}=p \sin \theta
$$

It is from the transverse momentum imbalance in collisions that the presence of neutrinos is inferred. These tools are needed to discuss the separate subsystems of the $\mathrm{D} \varnothing$ detector.

\subsubsection{Central Tracking System}

The closest sub-detector to the interaction point at $\mathrm{D} \varnothing$ is the central tracking system. It is split into two parts: The Silicon Microstrip Tracker (SMT) and the Central Fibre Tracker (CFT). These two components are surrounded by a $2 \mathrm{~T}$ solenoid magnet providing a field parallel to the beam line. This causes charged particles to move along a curved path inside the tracking system with a curvature proportional to the inverse of the particle momentum. A schematic of the central tracking system is shown in Figure 3.3 [15].

\section{Silicon Microstrip Tracker}

The innermost component of the tracking system is the SMT. The SMT consists of six barrel segments that are $12 \mathrm{~cm}$ long with concentric layers of silicon wafers. Arranged to ensure maximum coverage, the barrels provide measurements of central tracks up to $\left|\eta_{d}\right|<1.1$. In addition to the barrels there are so called F-disks and H-disks. F-disks comprise twelve double sided, overlapping silicon 


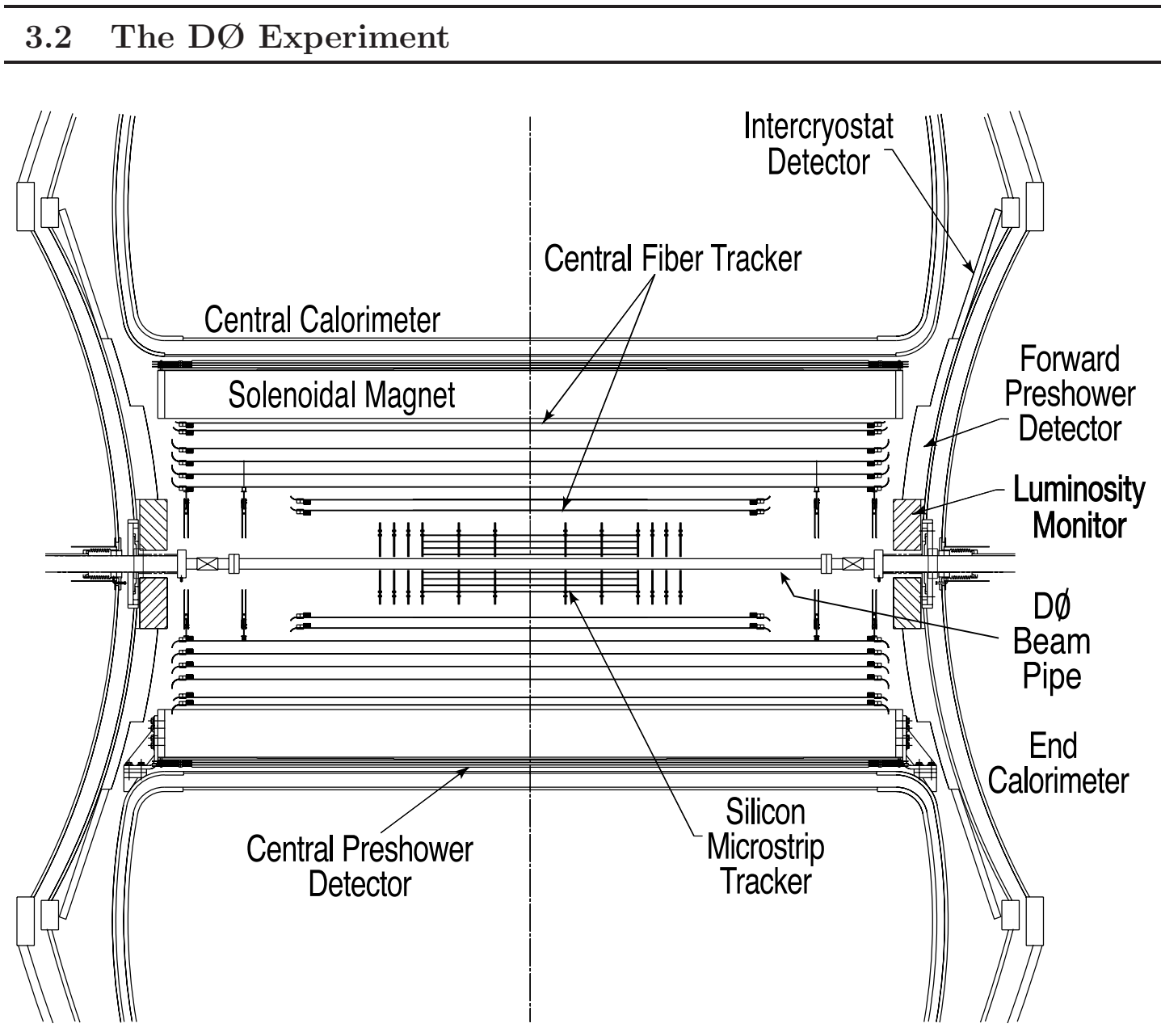

Figure 3.3: Diagram of the Central Tracking System [15].

wedges creating an annulus of inner radius $2.6 \mathrm{~cm}$ and outer radius $10.5 \mathrm{~cm}$. There are a total of twelve F-disks aligned with the barrels in the SMT. H-disks comprise 16 single sided, overlapping silicon wedges creating an annulus of inner radius $9.5 \mathrm{~cm}$ and outer radius $26 \mathrm{~cm}$. The $\mathrm{F}$-disks and H-disks together extend the coverage of the SMT to $\left|\eta_{d}\right|<3.0$. A diagram of the SMT detector is shown in Figure 3.4 [15].

The SMT wafers rely on n-type/p-type interface to create electron hole pairs via the ionisation caused by charged particles in the detector. An applied voltage then pulls these electrons to conducting readout strips which are interspersed between the silicon to read out the signal. 


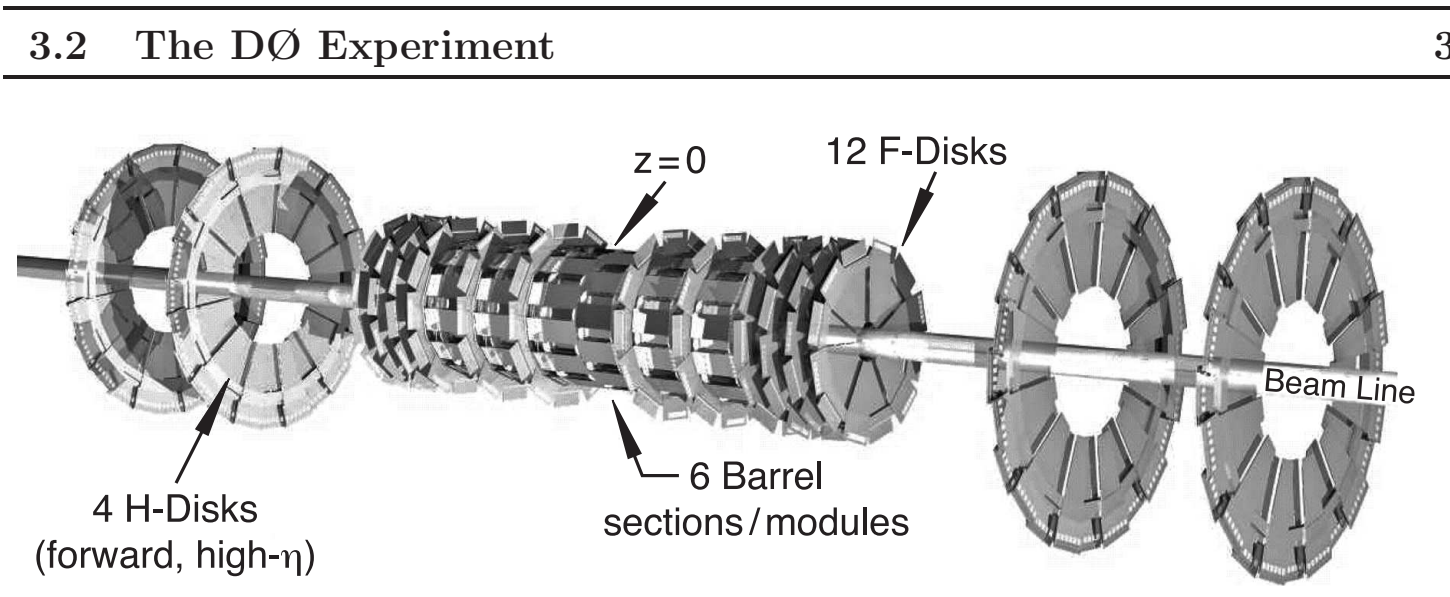

Figure 3.4: Diagram of the SMT [15].

\section{Central Fibre Tracker}

Outside of the SMT, with a coverage up to $\left|\eta_{d}\right|<2.0$, is the Central Fibre Tracker (CFT). The combination of data from the SMT and CFT allows for better tracking measurements in the detector. The CFT consists of eight carbon fibre cylinders holding scintillating fibres. A doublet structure of fibres is constructed with axial fibres being parallel to the beam line direction, odd cylinders contain a stereo fibre at an alternating angle of $3^{\circ}$. Together the axial and stereo fibres provide a measurement of $z$ for three-dimensional track reconstruction.

Each of the CFT's 71,680 fibres has a $775 \mu \mathrm{m}$ polystyrene core doped with fluorescing molecules with emission peaked at a wavelength of $535 \mathrm{~nm}$. Around the core there are two $15 \mu \mathrm{m}$ layers of cladding to increase the light collecting efficiency.

As charged particles pass through a fibre they cause scintillation. The fibres have a mirror at one end to reflect the light back to the other end of the fibre. At this end the fibre is joined to a clear fibre which allows the light to pass to a Visible Light Photon Converter (VLPC) [16]. The VLPC converts the light to electron hole pairs and these are collected by a $6 \mathrm{~V}$ bias voltage. To reduce the effects of noise in the process, the VLPC components are kept in liquid helium. Figure $3.5[17]$ shows one quarter of the inner D $\varnothing$ detector including the central 
fibre tracker.

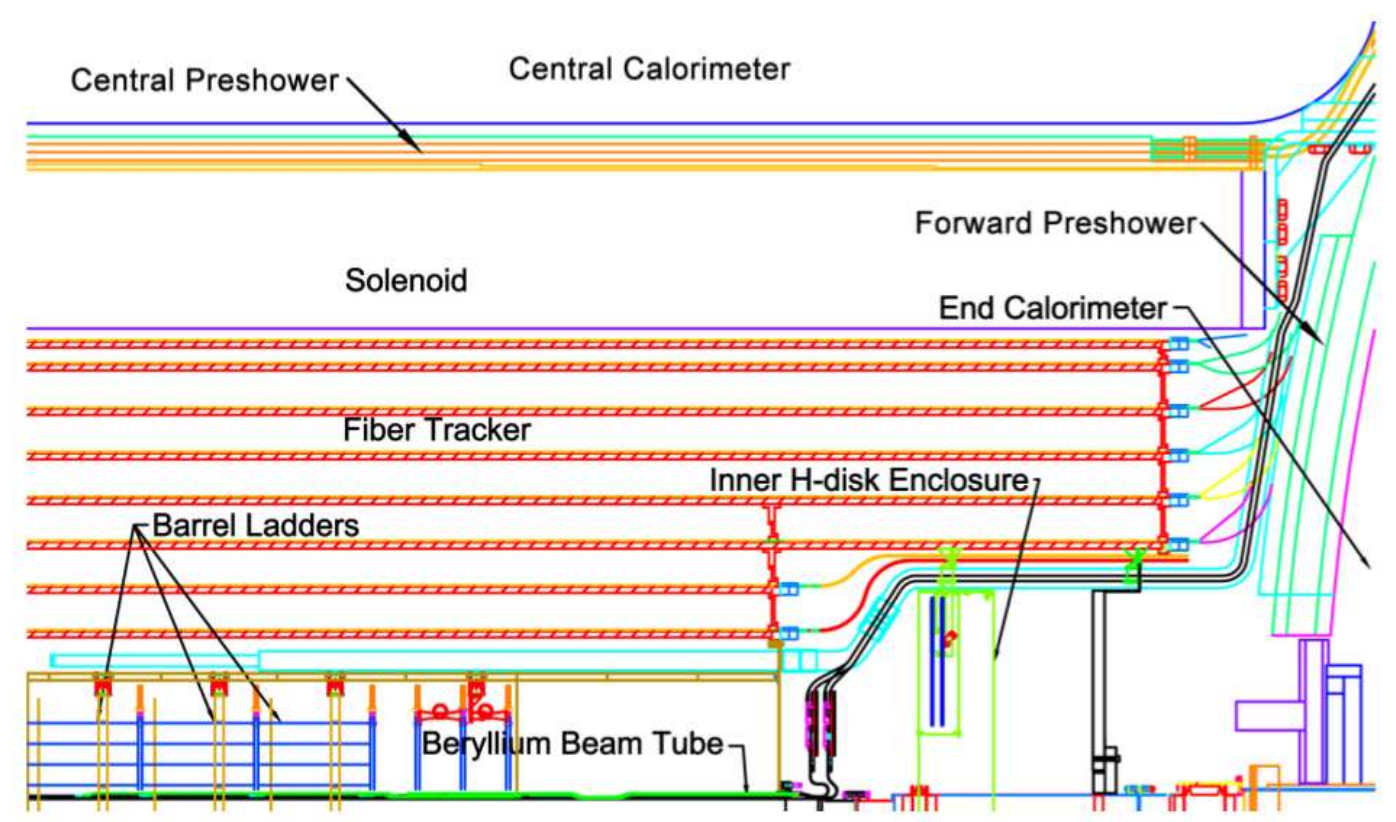

Figure 3.5: Diagram showing one quarter of the inner DØ detector including the CFT [17].

\subsubsection{Central and Forward Preshower Detectors}

It is possible for the particles to interact with material of the solenoid outside the central tracking system before they arrive at the calorimeter. Therefore, preshower detectors are installed in this region to improve calorimetry and improve tracking measurements. The Central Preshower Detector (CPS) is cylindrical and consists of three layers of scintillating strips, providing coverage to $\left|\eta_{d}\right|<$ 1.2. Inner strips are arranged axially and the top outer layers are arranged in a stereo configuration at angles of $23^{\circ}$. The Forward Preshower Detector (FPS) contains only two stereo layers of scintillation strips mounted on the inner edge of the calorimeter cryostats. Both the CPS and FPS are read out by passing light to the VLPC as in the CFT. 


\subsubsection{Calorimeter}

The calorimeter resides outside of the central tracking and preshower detectors and is designed to measure EM and hadronic energies. The interaction of particles with the matter of the calorimeter causes secondary particle showers which lose energy through ionisation of the calorimeter material. Once the showering process has been contained, an energy measurement can be made. The D $\varnothing$ calorimeter uses liquid argon as the active medium and depleted uranium (for the EM calorimeter) as an absorbing material.

The Calorimeter has a modular design to allow access to the inner parts of the detector and is shown in Figure 3.6 [15]. The calorimeter is separated into three modules: The Central Calorimeter (CC) with coverage to $\left|\eta_{d}\right|<1.2$ and two End Cap (EC) calorimeters extending the coverage to $\left|\eta_{d}\right|<4.5$. These modules are further split into three parts. Closest to the centre of the detector is the EM layer consisting of four layers of $3 \mathrm{~mm}$ thick depleted uranium absorber. Moving outwards is the Fine Hadronic (FH) layer of the calorimeter consisting of three (four) layers of $6 \mathrm{~mm}$ thick uranium-niobium absorber for the CC (EC) modules. Furthest out is the Coarse Hadronic $(\mathrm{CH})$ layer consisting of one $46.5 \mathrm{~mm}$ absorber made from copper (steel) for the CC (EC) modules.

Each of the calorimeter layers consists of readout cells of dimensions $0.1 \times 0.1$ in $\Delta \eta \times \Delta \phi$ and these cells are grouped into radial towers of dimensions $0.2 \times$ 0.2. The third layer of the EM calorimeter has a finer granularity of $0.05 \times 0.05$ in $\Delta \eta \times \Delta \phi$ as this is where the shower maximum occurs. A diagram showing the tower structure of the calorimeter is shown in Figure 3.7 [15]. The readout cells consist of a group of adjacent unit cells which are immersed in the liquid argon of the calorimeter. Showering particles in the calorimeter ionise liquid argon and electrons are drawn to a resistive coating which is held at a high voltage $(2.5 \mathrm{kV})$. Charge is induced on the copper pad of the unit cell which is proportional to the 


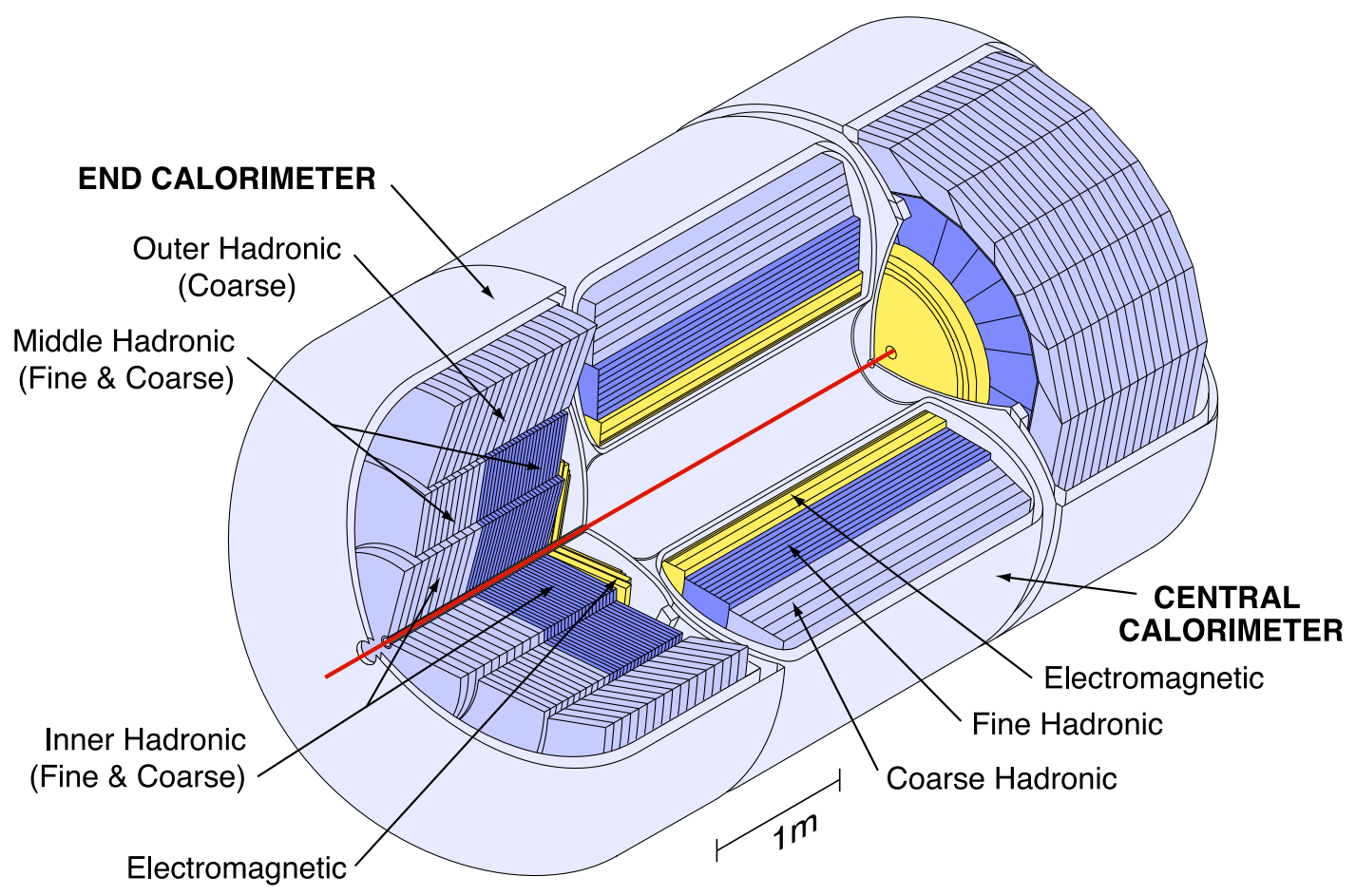

Figure 3.6: Diagram of the Calorimeter [15].

energy deposited. Readout electronics convert this to an analogue signal for recording.

The energy resolution of the calorimeter is different for the EM and hadronic layers and beam tests were carried out on the calorimeter to determine the following resolutions $(E$ in $\mathrm{GeV})[18]$ :

$$
\begin{gathered}
E M: \frac{\sigma_{E}}{E}=\frac{0.15}{\sqrt{E}}+0.003, \\
\pi^{ \pm}: \frac{\sigma_{E}}{E}=\frac{0.45}{\sqrt{E}}+0.04
\end{gathered}
$$

\subsubsection{Inter Cryostat Detector}

The region between $1.1<\left|\eta_{d}\right|<1.4$ is the Inter Cryostat Region and is largely uninstrumented, but the material can still cause particle showering. To facilitate 


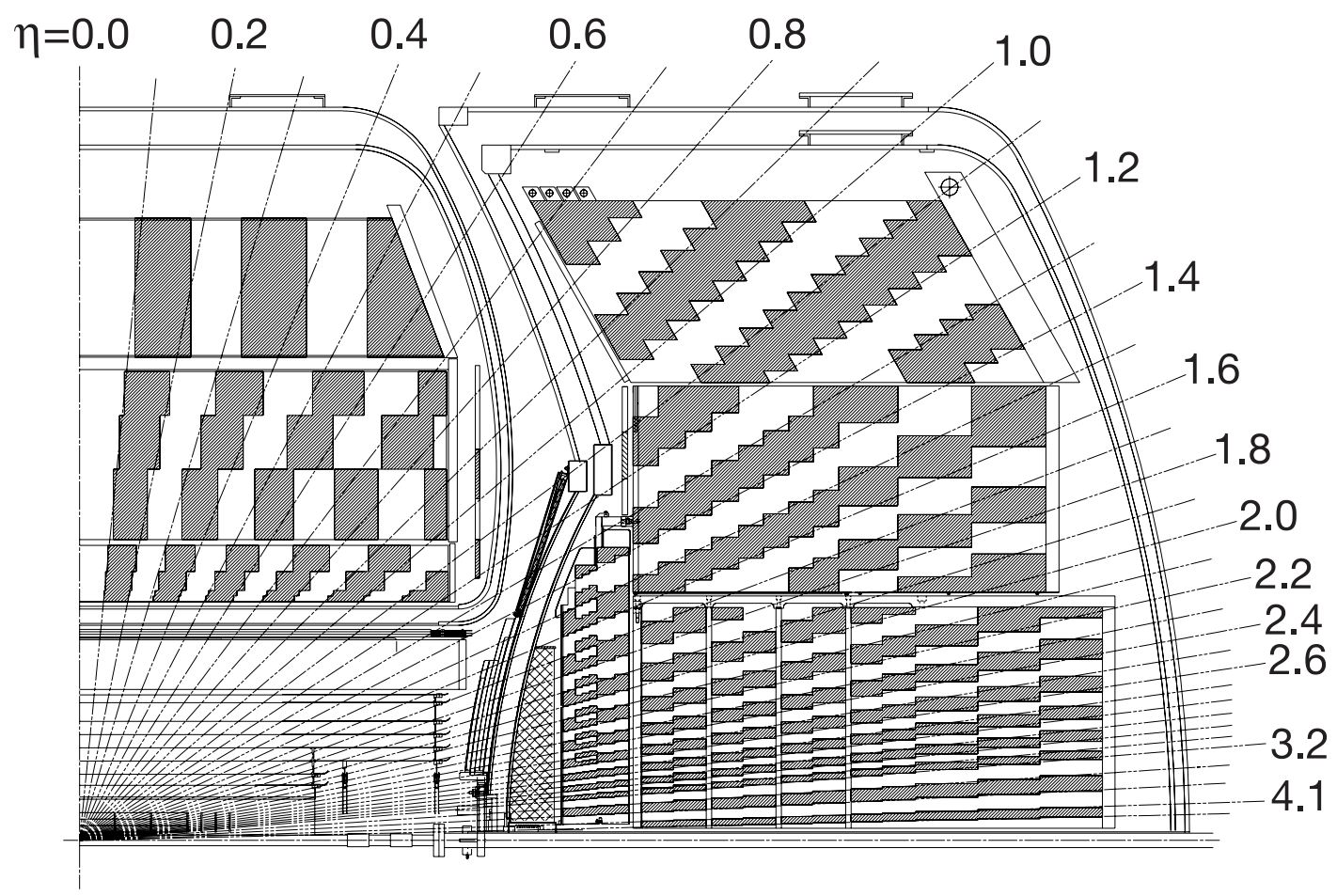

Figure 3.7: Diagram of a quadrant of the Calorimeter [15].

particle detection in this region, scintillating detectors are mounted on the EC cryostat walls. There are 384 scintillating tiles each $0.1 \times 0.1$ in $\Delta \eta \times \Delta \phi$. In addition to these tiles, there are separate single liquid argon cell structures without absorber material, known as massless gaps, which allow a measurement of shower formation in the gap region. Figure 3.8 [15] shows a cross-section of the inter cryostat detector (ICD) with the ICD tiles on the endcap cryostats.

\subsubsection{Muon System}

The muon system is the outer-most subsystem of the $\mathrm{D} \varnothing$ detector as muons pass through the rest of the detector mostly unimpeded. The muon system is made up of a 1.8 T toroidal magnet, a Wide Angle Muon Spectrometer (WAMUS) for the central region and a Forward Angle Muon Spectrometer (FAMUS) for the 


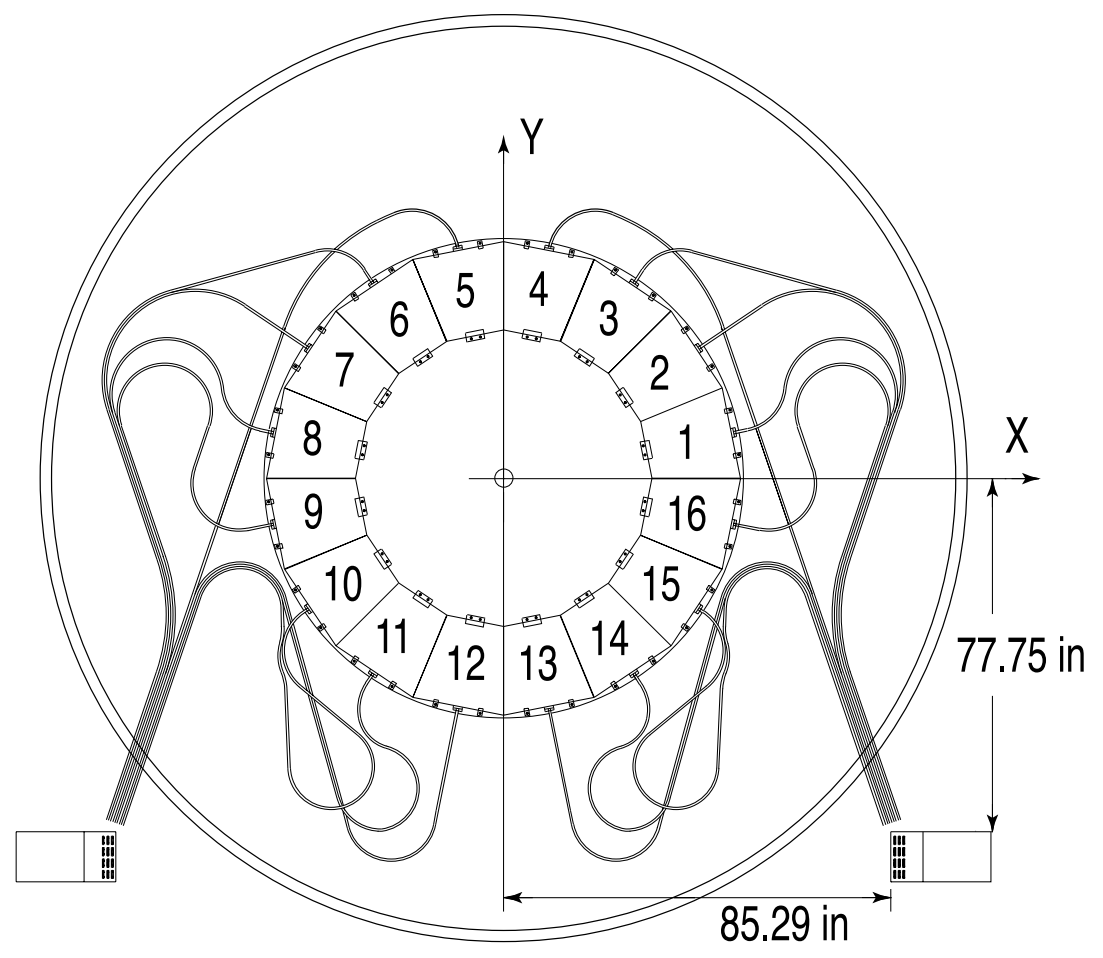

Figure 3.8: Diagram showing a cross-section of the ICD [15].

forward region.

The toroidal magnet acts as additional shielding to prevent hadronic activity passing to the muon detector, and as another method of measuring the muon momentum. However, track measurements from the inner detector usually have much better resolution.

The WAMUS comprises proportional drift tubes (PDTs) and scintillation tiles which are arranged in three layers (A, B and $\mathrm{C}$ ). The A layer is located inside the toroid whereas the $\mathrm{B}$ and $\mathrm{C}$ layers are outside. The FAMUS has a similar structure but with Mini Drift Tubes (MDTs) and scintillator pixels. An exploded view of the drift tube and scintillator systems is shown in Figures 3.9 [15] and $3.10[15]$.

The drift tubes are rectangular aluminium tubes containing a wire anode at the centre and cathode pads above and below the wire. The tubes are filled with 
a gas of $84 \%$ argon, $8 \%$ methane and $8 \% \mathrm{CF}_{4}$ for PDTs and $90 \% \mathrm{CF}_{4}$ and $10 \%$ methane for MDTs.

There are 94 PDT chambers in the WAMUS arranged in a cubic structure 24 cells wide per chamber. Scintillation counters are located on the outer layer of the top and bottom of the muon PDTs known as cosmic gap and cosmic bottom counters. Additionally, there are $A \phi$ scintillation counters covering the A layer PDTs. These counters are used in the trigger system. There are three layers of MDTs in the FAMUS which are further divided into octants and each of these layers contain three (four) drift tubes in the B,C (A) layers respectively.

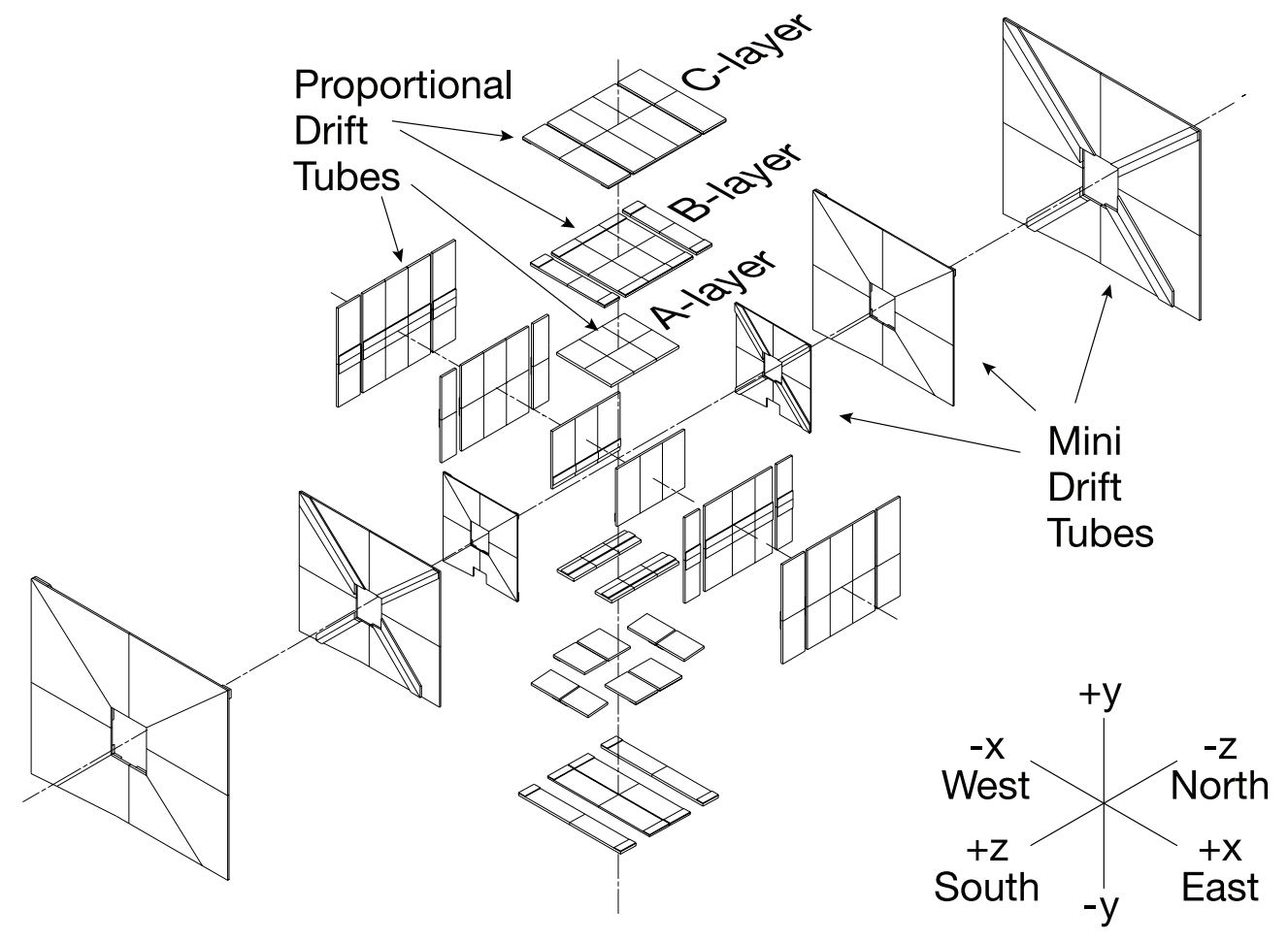

Figure 3.9: Exploded view of muon drift tube system [15].

\subsubsection{Luminosity Monitor}

The instantaneous luminosity is defined as the number of colliding particles per unit area per unit second. It is necessary to know the luminosity to understand 


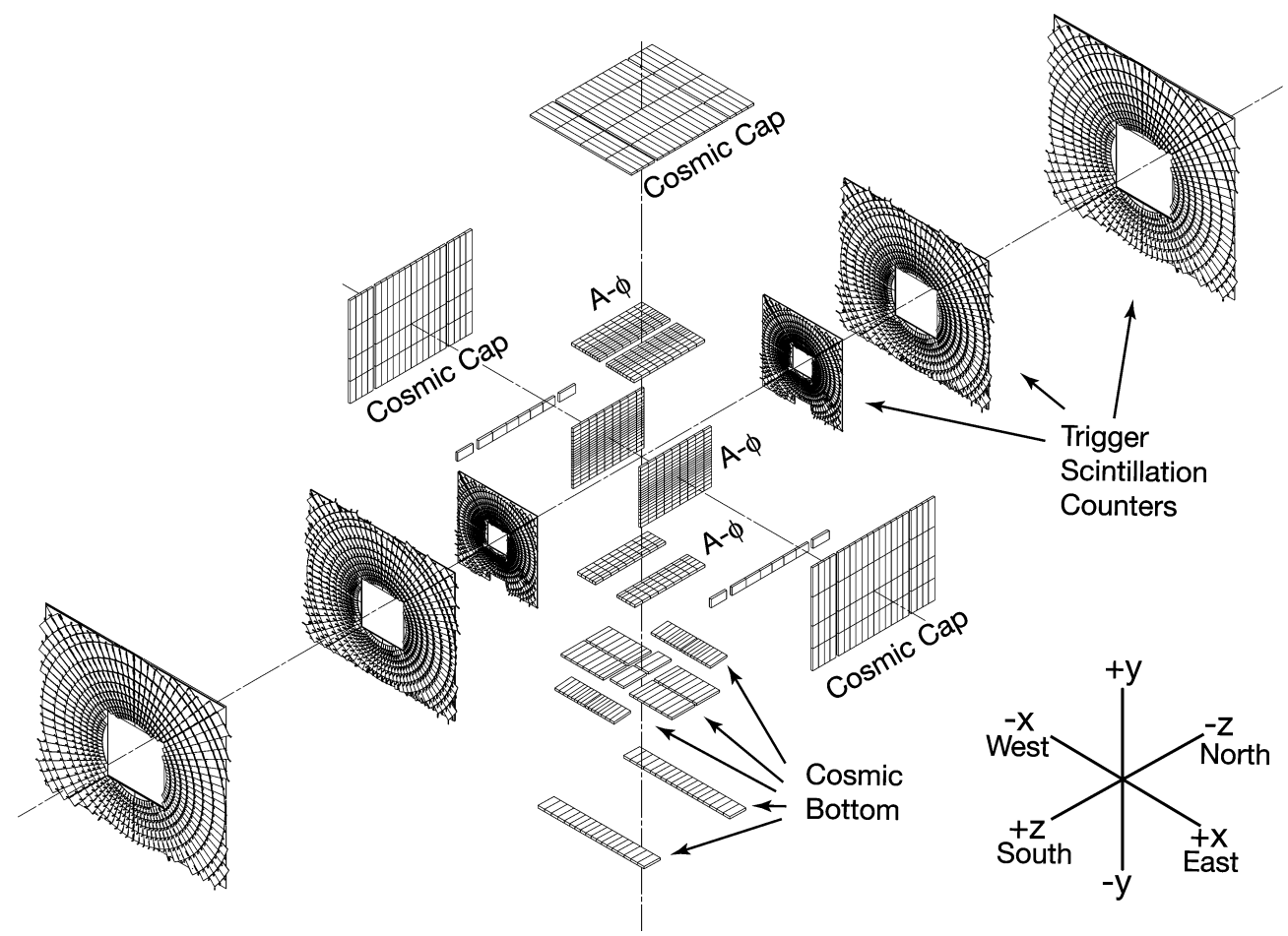

Figure 3.10: Exploded view of muon scintillator system [15].

the yield of events in the detector and to measure cross-sections. Luminosity monitors are designed to measure the instantaneous and integrated luminosity being delivered to the experiment. They comprise 24 wedges of scintillators at $z= \pm 140 \mathrm{~cm}$ covering $2.4<\left|\eta_{d}\right|<4.4$ and measure the rate of inelastic $p \bar{p}$ collisions. The scintillators produce photons when particles interact and these photons are detected by photo multiplier tubes (PMTs) which are mounted on the scintillators. To determine the luminosity accurately, measurements of the time of flight are necessary to distinguish $p \bar{p}$ interactions from beam halo interactions. It is also necessary to determine the frequency of multiple $p \bar{p}$ collisions per beam crossing so that the luminosity can be correctly calculated. The instantaneous luminosity decreases exponentially over the course of a physics store and an accurate understanding of the instantaneous luminosity is necessary to optimise running conditions. A plot of the instantaneous luminosity from a typical store 
is shown in Figure 3.11 [19]. The dashed purple line is the instantaneous luminosity, the left axis is the trigger rate $(\mathrm{Hz})$ and the right axis is the instantaneous luminosity. The changes in the trigger rate over the course of the store are due to optimisation of trigger system at the beginning of a new data taking run. This allows the highest rate of data taking for a given instantaneous luminosity.

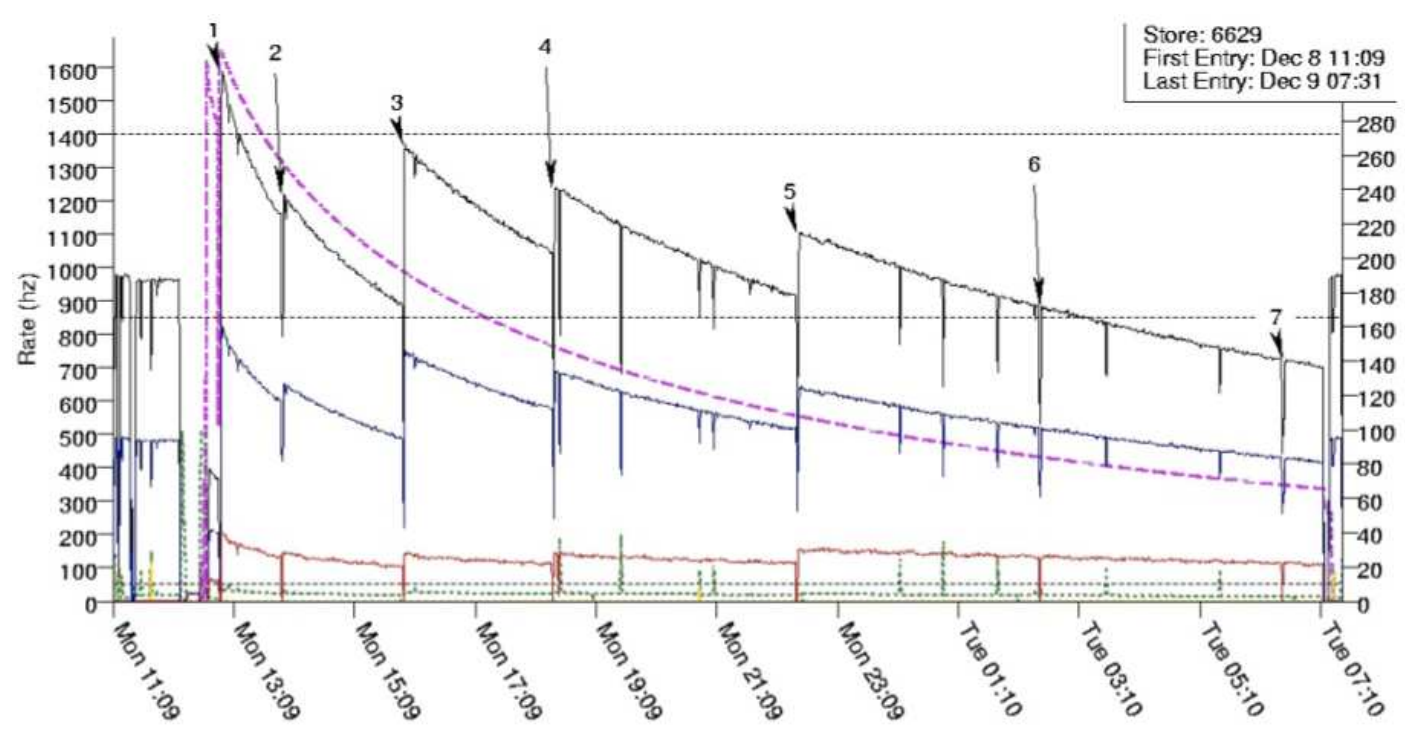

Figure 3.11: A typical store at D $\varnothing$. The left axis shows the trigger rate $(\mathrm{Hz})$ and the right axis shows the instantaneous luminosity $\left(\mathrm{cm}^{-2} \mathrm{~S}^{-1}\right)$ [19]. The continuous dashed purple line indicates the instantaneous luminosity during the store and the other three lines indicate the

Level 1, 2 and 3 trigger rates with Level 1 the highest rate and Level 3 the lowest rate.

\subsubsection{Trigger System}

Collisions occur at a rate of $\sim 1.8 \mathrm{MHz}$ and it is impossible for the experiment to collect and store all of the information from these events. It is therefore necessary to implement a selection mechanism giving priority to those events that are of most interest to the physics programme at D $\varnothing$. This trigger system is designed to select a wide range of physics processes with signatures such as: High transverse momentum particles with rejection of much of the copious $p \bar{p}$ background for electroweak and QCD measurements, lower momentum tracks for B physics as 
well as zero bias events triggered to understand noise effects in the detector. The rate at which the three trigger levels are able to accept data and associated data acquisition elements are shown in Figure 3.12 [15].

The trigger system is split into three distinct levels (Level 1, 2 and 3). Level 1 (L1) consists of hardware trigger elements that make decisions in real time about whether to pass the event and is able to pass around 2000 events to Level 2 (L2) per second. A global L1 accept can be one or more individual terms from the tracking (not SMT), calorimeter or muon sub-detector trigger systems. The L2 trigger system is designed to reduce the rate down to around $1000 \mathrm{~Hz}$ and has access to all detector sub-systems. The Level 3 (L3) trigger system comprises a farm of offline computer processors that run an fast version of the reconstruction algorithms to determine whether the event is to be written to storage media. The experiment is able to write to tape around 100 events per second.

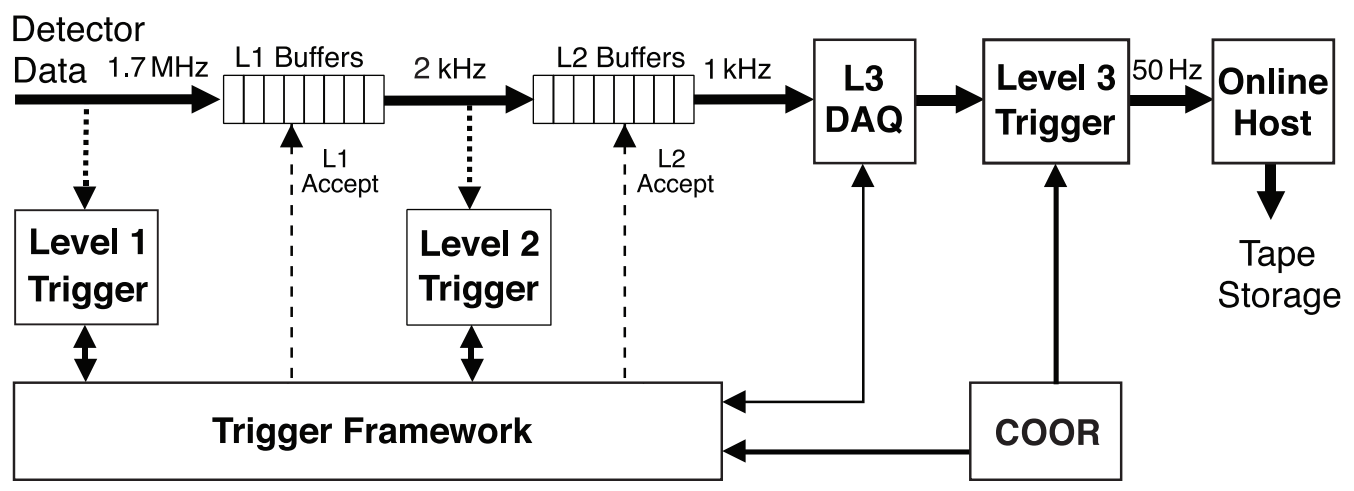

Figure 3.12: Trigger system and data storage flow diagram [15].

\section{Data Acquisition}

Figure 3.13 [19] shows that the data collected since the beginning of RunII (April 2002) has now exceeded $6 \mathrm{fb}^{-1}$. These data are split into two run periods denoted RunIIa and RunIIb as illustrated. During the shutdown period between RunIIa 


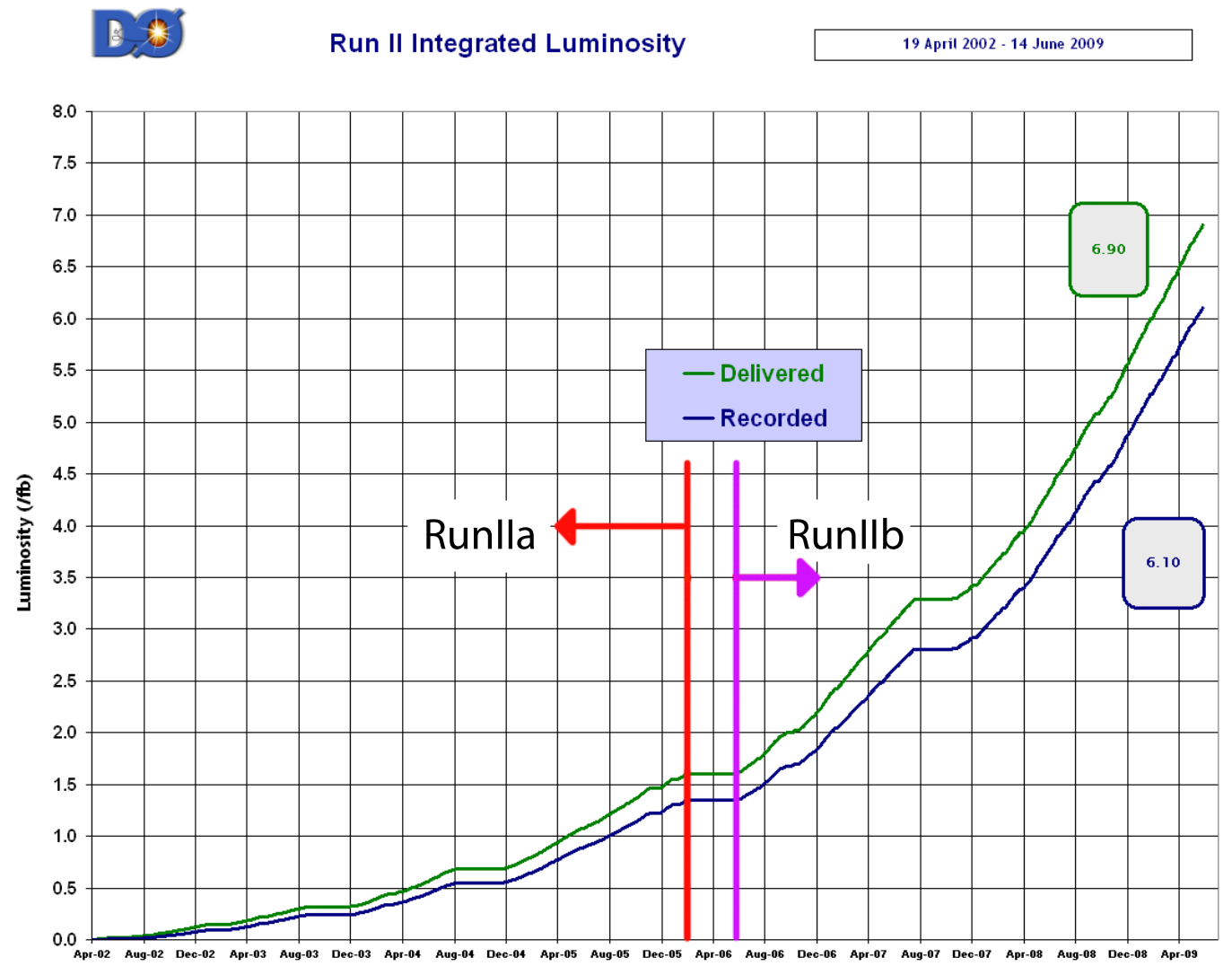

Figure 3.13: Integrated luminosity accrued in RunII of the DØ experiment showing the RunIIa and RunIIb data periods [19].

and RunIIb significant upgrades were made to the $\mathrm{D} \varnothing$ detector as documented in [15]. The data used in the analysis described in this thesis use RunIIa plus RunIIb data up to January 2009 yielding a total data set of $4 \mathrm{fb}^{-1}$ after data quality requirements are made.

Before an analysis is completed the quality of the data needs to be assessed. Events are flagged if there were problems with certain parts of the detector during that run, such as hot calorimeter cells or missing detector components. Depending on the type of analysis, a decision is made to determine whether this run is suitable to retain in the analysis or whether the quality has been sufficiently compromised to remove the run. Additionally, luminosity blocks, which are the fundamental unit of time measurement for integrated luminosity can be marked as bad if the 
instantaneous luminosity was not accessible due to a detector failure or problem with the luminosity monitoring system. These luminosity block numbers (LBNs) are flagged so they can be removed in analyses. 


\section{Chapter 4}

\section{Analysis Tools}

\subsection{Reconstruction}

This chapter describes the algorithms that are used to reconstruct leptons and jets, to identify jets from b-quarks and to determine the missing transverse energy from neutrinos. All of these objects are essential for reconstructing the $W H \rightarrow$ $\tau \nu b \bar{b}$ decays and to separate them from background.

\subsubsection{Lepton Identification}

The main lepton of interest to this analysis is the hadronically decaying tau lepton. However, selections have to be made on electrons and muons to remove them from the analysis to avoid double counting events from dedicated Higgs searches where the associated $W$ or $Z$ boson decays into electrons or muons. Another important reason to focus on electron identification, is that electrons have a very similar detector signature to single-pronged hadronic tau decays, so it is possible for an object to be reconstructed as both an electron and a hadronically decaying tau. 


\section{Electron and Photon Identification}

Electrons and photons have very similar signatures in the calorimeter, and the main discrimination between the two is the requirement of a track pointing to the location of the object in the calorimeter for an electron. For a cluster of energy to be reconstructed as an EM object it is required to pass various shower shape, energy and quality constraints based upon the energy deposited in a cone of radius $\Delta R=0.4\left(E_{R<0.4}\right)$. To distinguish the EM signature from hadronic activity in the calorimeter, the energy deposited in the EM layers of the calorimeter in a cone of $\Delta R=0.2\left(E_{R<0.2}\right)$ is required to be $90 \%$ of the total cluster energy. Additionally, to reduce the contribution from jets, the energy deposit is required to be isolated,

$$
I=\frac{E_{R<0.4}-E_{R<0.2}}{E_{R<0.2}}<0.15 .
$$

Once these conditions have been satisfied, a multivariate technique is used to combine the discriminating power of variables such as the energy deposits in each of the four EM layers, the fractional energy deposit in the fine hadronic and preshower detectors and the shower width into one discriminant known as the "H-matrix" [20]. The H-matrix provides a measure of the likelihood that the signature has arisen from an electron or a photon and different selections are placed on the output of this discriminant depending upon the purity and efficiency desired.

\section{Muon Identification}

Muons are reconstructed from hits in the muon system's wire chambers and scintillators. A local muon is defined as a muon that only has muon sub-system information, whereas a central muon is required to additionally be matched to 
a central track. Wire chambers and scintillators in the A or B,C layers are combined into segments and segments in different layers are used to construct a fit for the muon's trajectory through the detector. The $n_{\text {seg }}$ variable [21] is used to categorise the layers in which the muon has left a signature in the following way:

- $\left|n_{\text {seg }}\right|=0$; single muon hit matched to a central track

- $\left|n_{\text {seg }}\right|=1$; local muon with an A layer segment only

- $\left|n_{\text {seg }}\right|=2$; local muon with a B,C layer segment only

- $\left|n_{\text {seg }}\right|=3$; local muon with an A and B,C layer segment

The quality of the muon fit is defined by the following operating points:

- Loose - One scintillator hit in A or B,C layers with at least two wire chamber hits in one of the layers.

- Medium - Two wire chamber hits in each of the A and B,C layers each requiring a matching scintillator hit.

- Tight - In addition to Medium, three wire chamber hits in the B,C layers with a matching scintillator hit. The fit for a central track match must have converged.

To reduce the effect of charged pions punching through the calorimeter and faking muons and hadronic decays $(\pi \rightarrow \mu)$, isolation requirements are made. They are separated into Halo and Track Isolation in the following way:-

- Halo Isolation - The transverse energy deposited in the calorimeter in a halo $0.1<R<0.4$ around the muon axis is required to be less than $2.5 \mathrm{GeV}$. 
- Track Isolation - The sum of the transverse momentum of all tracks within a cone $\Delta R=0.5$ around the muon is required to be less than $2.5 \mathrm{GeV}$.

To reduce the effect of cosmic rays that can penetrate the detector and leave multiple signatures in the muon system, a timing requirement is made such that scintillator hits too far away from that expected from collisions are removed. A requirement can also be made on the distance of closest approach (dca) of the muon to the primary vertex as cosmic rays will be spread across the whole detector and not just around the collision point.

\section{Hadronic Tau Identification}

Hadronic tau decays are identified via central tracks and calorimeter clusters. The calorimeter clusters are formed inside a cone $\Delta R=0.3$. A nearest neighbour algorithm is then used to find electromagnetic sub-clusters in the third layer of the EM calorimeter (the third layer has the finest granularity). These subclusters are used to identify neutral pions $\left(\pi^{0} \rightarrow \gamma \gamma\right)$ from hadronic tau decays. Requirements are made that tracks are associated with the calorimeter cluster. Each of the tracks with $p_{T}>1.5 \mathrm{GeV}$ that reside within a cone $\Delta R=0.5$ are ordered in $p_{T}$, the highest $p_{T}$ track and up to two additional tracks (if within $2 \mathrm{~cm}$ at dca) are associated with the cluster. If the invariant mass calculated using the first and second track is less than $1.1 \mathrm{GeV}$ then a second track is added, and if the mass of the three tracks is below $1.7 \mathrm{GeV}$, the third track is added [22]. Once this basic reconstruction has taken place, the tau candidates are split into types defined by their detector signature which are based loosely on the known hadronic decay modes of a tau (see Table 2.3), in the following way:

- Type 1 - One track and no EM sub-cluster.

- Type 2 - One track and at least one EM sub-cluster. 
- Type 3 - More than one track, with or without EM sub-clusters.

Electrons are likely to be reconstructed as type 2 taus. However, the largest number of fake taus occur from the copious amount of hadronic multi-jet activity from $p \bar{p}$ collisions. To deal with this, a number of neural networks [23] are trained to distinguish jets and electrons from hadronic tau decays. A large number of input variables to the neural networks are considered as the decay signature for each tau type is different. A subset of these variables is found to give the best discriminating power in each neural network. Variables considered for the tau neural networks are presented in the following list:

- $\left(E^{E M 1}+E^{E M 2}\right) / E^{\tau}$, where $E^{E M 1}$ and $E^{E M 2}$ are the energies deposited in the first and second layers of the EM calorimeter, respectively, and $E^{\tau}$ is the total energy of the tau.

- $\Sigma p_{T}^{\text {trk }} / \Sigma p_{T}^{\tau_{t r k}}$, where $p_{T}^{\text {trk }}\left(p_{T}^{\tau_{t r k}}\right)$ is the $p_{T}$ of a track (tau track) within $\Delta R$ $=0.5$.

- The fraction of the tau energy in the fine hadronic section of the calorimeter.

- $E_{T}^{\tau} /\left(E_{T}^{\tau}+\Sigma p_{T}^{\tau_{t r k}}\right)$, which is the transverse energy of the tau cluster divided by the transverse cluster energy plus the sum of the track transverse momenta.

- $\sqrt{(\Delta \phi \sin \theta)^{2}+(\Delta \eta)^{2}} / \pi$, where $\Delta \phi(\Delta \eta)$ is the difference in $\phi(\eta)$ of the vector sum of tau tracks and the vector sum of EM clusters.

- Transverse energy of leading EM sub-cluster divided by the transverse energy in layer 3 of the calorimeter in $R=0.5$.

- $\left(E_{T}^{\text {tow } 1}+E_{T}^{\text {tow } 2}\right) / E_{T}^{\tau}$, where $E_{T}^{\text {tow } 1}\left(E_{T}^{\text {tow } 2}\right)$ is the transverse energy of the leading (next to leading) calorimeter towers. 
- $E_{T}^{e m} / E_{T}^{\tau}$, where $E_{T}^{e m}$ is the transverse energy of the EM sub-clusters.

- Transverse momentum of leading tau track divided by the transverse energy of the tau candidate.

- $\left(E_{T}^{\tau}-E_{T_{\text {core }}}^{\tau}\right) / E_{T_{\text {core }}}^{\tau}$, where $E_{T_{\text {core }}}^{\tau}$ is the transverse energy of the tau cluster inside $\Delta R=0.3$, compared to $E_{T}^{\tau}$ which uses $\Delta R=0.5$.

- $\sqrt{\sum_{i=1}^{n}\left[\left(\Delta \phi_{i}\right)^{2}+\left(\Delta \eta_{i}\right)^{2}\right] E_{T_{i}} / E_{T}}$, where $i$ sums over the towers in the cluster.

- Detector $\eta$ of the tau candidate scaled to be between 0 and 1 for the neural network training.

A separate neural network is trained for each of the tau types. Additionally, type 1 taus have a dedicated neural network for the ICD (see Section 3.2.5) and type 2 taus have a dedicated electron - tau separating neural network. The ICD region is susceptible to electron fakes in type 1 tau candidates, when an electron passes close to a crack in the calorimeter. Their detector signature is different to type 1 tau candidates in the central part of the detector, and motivates a dedicated training and neural network.

The training sample for the tau neural networks are simulated $Z \rightarrow \tau \tau$ events. The backgrounds are estimated from anti-isolated $\mu+\tau$ data events for the hadronic tau - jet separating neural networks,. These events exhibit the same topology and similar kinematics as $Z \rightarrow \tau \tau$ events but the inverted muon isolation criteria increases the likelihood that the event arises from a di-jet fake. Simulated $Z \rightarrow e e$ events are used to train the type 2 electron - tau separating neural network.

A cut is placed on the neural network output for each tau type dependent upon the signal acceptance versus background rejection required. Figure 4.1 shows the signal acceptance and background rejection for the type 2 hadronic 
tau - jet separating neural network; where p17 and p14 are different versions of the reconstruction software. Placing a cut around 0.9 on the neural network output will reduce the background to around 5\% while preserving around $60 \%$ of the signal, thus increasing the purity of hadronic tau decays over jet fakes considerably. Full details of training procedures and results can be found in the documentation [23].

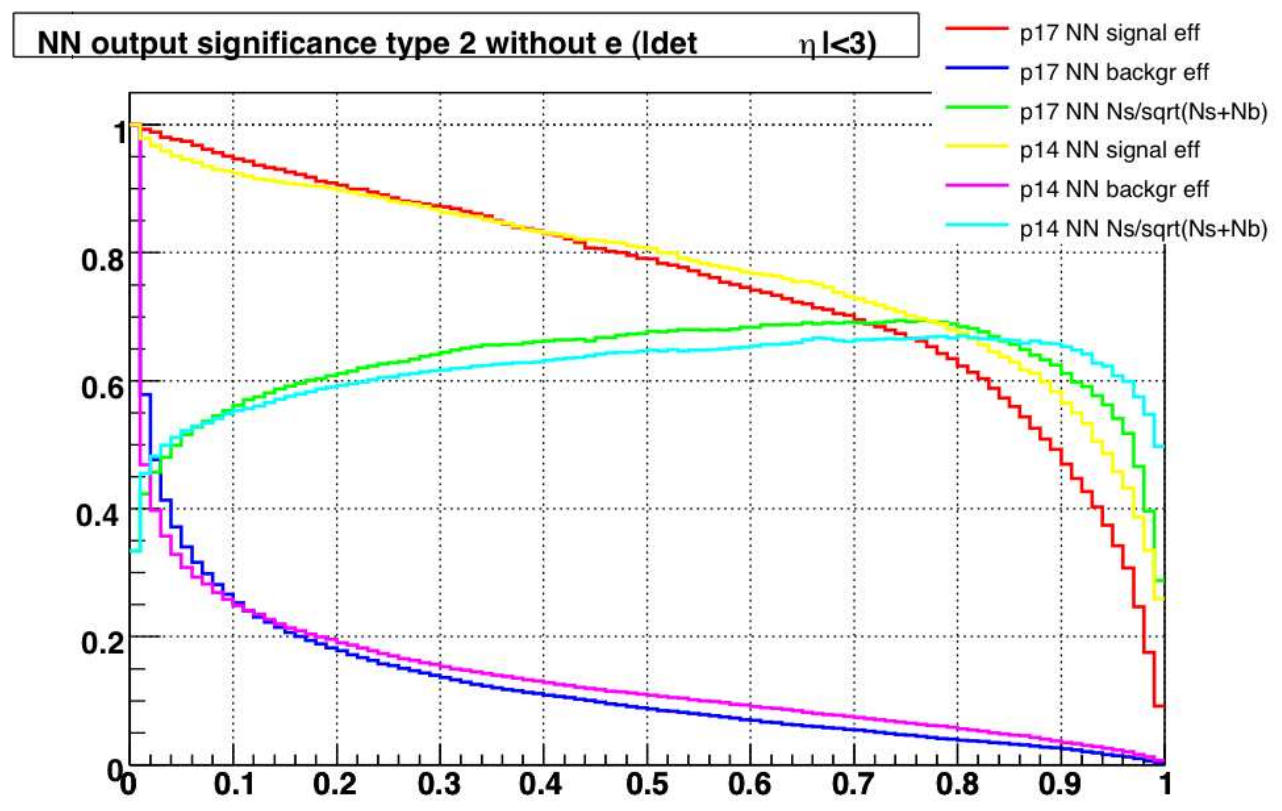

Figure 4.1: Signal acceptance and background rejection for type 2 taus as a function of the cut applied to the tau neural network output [23].

The hadronic tau neural network has been used to yield very pure $Z \rightarrow \tau \tau$ samples where one tau decays hadronically and the other leptonically. A $Z \rightarrow \tau \tau$ cross-section measurement has been published using these tools [24]. A search for a supersymmetric Higgs boson in the $\tau \tau$ final state also uses these tools. Figure $4.2[25]$ shows the visible mass $\left(M_{v i s}=\sqrt{\left(P_{\tau 1}+P_{\tau 2}+P_{\mathrm{T}}\right)^{2}}\right)$ distribution for the three final states analysed and demonstrates how the neural network tools are used to obtain a sample dominated by hadronic taus. 


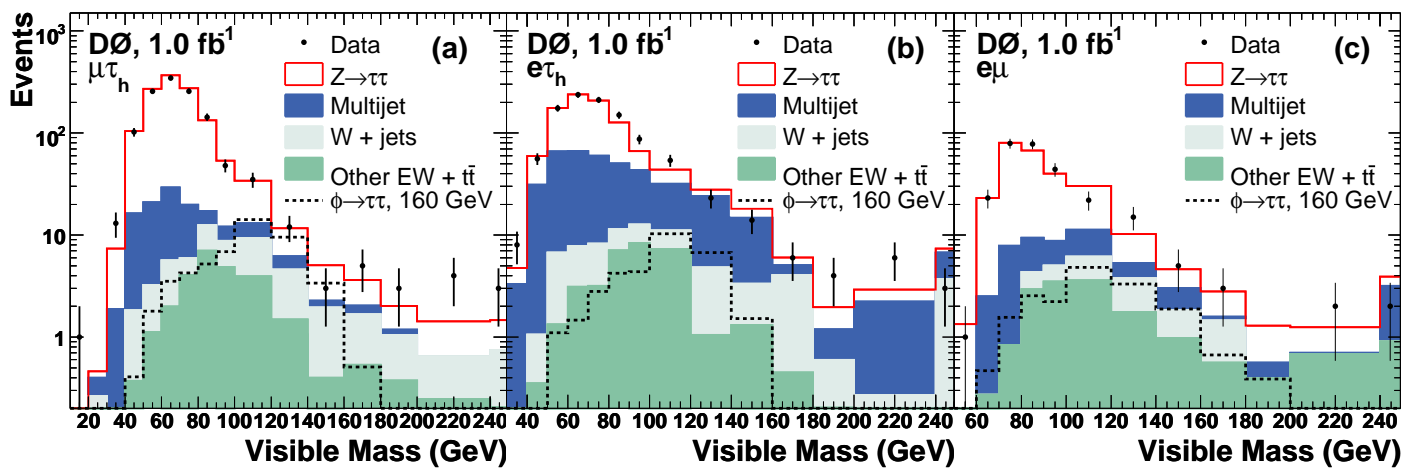

Figure 4.2: Visible mass distributions for the three final states analysed in the published $1 \mathrm{fb}^{-1} H \rightarrow \tau \tau$ result [25].

\subsubsection{Jet Identification}

Jets from hadrons dominate the detector in $p \bar{p}$ collisions. Jet identification is a multi-stage process. The two main components are the reconstruction algorithm and the determination of the jet energy scale.

\section{Jet Reconstruction}

The reconstruction of jets is an iterative process over calorimeter cells whose energy exceeds $0.5 \mathrm{GeV}$. A cone of radius $R=0.3$ is defined around such cells and if the energy inside this cone exceeds $1.0 \mathrm{GeV}$, then a larger cone of radius $R=0.5$ is created around the energy weighted centre. The cone is then centred around the new energy weighted centre; this procedure continues until stability is achieved. This is known as a proto-jet. Then adjacent proto-jets are considered and merged together if they share over half their energy across a cell boundary, otherwise they are split to form separate jets.

A number of quality criteria are imposed on these jets to account for calorimeter noise, hot cells in the calorimeter and EM fakes: The EM energy fraction of the jet must be greater than 0.05 to remove calorimeter noise but less than 0.95 to remove the EM fakes. The fraction of energy in the coarse hadronic part of the 
calorimeter must be less than 0.4 as the coarse hadronic calorimeter suffers from noise more greatly than the other parts. The ratio of the energies of the leading two calorimeter towers must be less than 10, and $90 \%$ of the jet energy must come from more than one cell to reduce the effect of hot cells in the calorimeter. Finally the transverse energy measured by the level 1 calorimeter trigger must be greater than $40 \%$ of the reconstructed jet energy in the EM and fine hadronic layers of the calorimeter.

\section{Jet Energy Scale}

For jets whose transverse energy is greater than $15 \mathrm{GeV}$ and $\left|\eta_{d}\right|<2.5$, corrections are made to their energy to bring them in line with the hadron level energy. The corrections to the jet energy scale take into account a number of different features which cause the jet energy to differ from the particle-jet energy [26]:

- Jets contain many different particles which have different calorimeter responses,

- Energy deposits from other $p \bar{p}$ interactions and calorimeter noise contribution to the measured energy,

- Not all showering particles deposit their energy in the jet cone.

\subsubsection{Missing Transverse Energy}

To infer the presence of neutrinos in events the energy imbalance is considered. It is only possible to calculate such an imbalance in the direction transverse to the beam pipe as the boost in the $z$ direction is not known. The missing transverse energy $\left(E_{\mathrm{T}}\right)$ is calculated from the $x$ and $y$ components of momentum $p_{x}$ and $p_{y}$ of the particles in the event in the following way: 


$$
E_{\mathrm{T}}=-p_{T}=\sqrt{\left(\sum_{i} p_{x_{i}}\right)^{2}+\left(\sum_{i} p_{y_{i}}\right)^{2}}
$$

where $i$ is the index over the reconstructed particles in the event. The $\mathbb{F}_{\mathrm{T}}$ is corrected at the analysis stage, once particle energy corrections have been made.

\section{Missing Transverse Energy Significance}

The missing transverse energy significance $\left(E_{\mathrm{T}_{\mathrm{SIG}}}\right)$ is calculated from the objects in the event and their resolutions [27]. It is calculated as the ratio of the $\mathbb{E}_{\mathrm{T}}$ to the uncertainty on the $\mathbb{F}_{\mathrm{T}}$ (which is calculated from the resolution of the objects that take part in the calculation of the $\left.\mathbb{E}_{\mathrm{T}}\right)$. It is used to determine how likely it is that an event contains "real" missing transverse energy or whether the missing transverse energy arises from mis-measurements in the detector. This measurement is dominated by the energy resolution of jet measurements which is derived from a sample of back-to-back di-jet events. However, in events with large mis-measurement the contribution from unclustered energy outside the jets, whose resolution is poorly understood, contributes most significantly. The missing transverse energy signifiance is therefore useful when attempting to remove poorly reconstructed events from the analysis sample.

\subsection{4 b-jet Identification}

The ability to distinguish b-jets from the overwhelming number of light quark jets produced in $p \bar{p}$ collisions is the key to search for a low mass Higgs boson.

\section{Vertex Confirmation}

In the RunIIb portion of the data an additional condition is imposed on jets before the b-tagging algorithms are applied. Vertex confirmation requires that 
at least two of the tracks from the jet are associated with the primary vertex of the event. This removes jets with tracks coming from interactions other than the primary interaction point which occur due to the higher instantaneous luminosity in RunIIb.

\section{Taggability}

Quality requirements are made of the jets selected in the analysis before b-tagging, defined as "taggability". A jet is defined to be "taggable" if it matches a track-jet in a cone of radius $\Delta R=0.5$, where a track-jet is defined by a cone clustering algorithm with tracks satisfying the following requirements:

- One or more seed tracks with $p_{T}>1 \mathrm{GeV}$,

- One or more additional tracks with $p_{T}>0.5 \mathrm{GeV}$,

- Tracks must have at least one SMT hit,

- Track's distance of closest approach to the primary vertex in the $x-y$ plane $\leq 0.2 \mathrm{~cm}$,

- Track's distance of closest approach to the primary vertex in the $z$ axis $\leq$ $0.4 \mathrm{~cm}$.

\section{b-tagging}

b-jets are identified by a number of different algorithms at D $\varnothing$. These are based mainly upon information from tracks in jets and secondary vertices in the event which occur from the decay of B-mesons traveling away from the primary interaction point. However, to maximise the signal to background ratio a neural network is trained on a combination of input variables from each of the b-tagging algorithms. The three main algorithms for b-jet identification are: 
- Jet Lifetime Probability (JLIP) - A tagger that combines the impact parameters (where the impact parameter is the minimal distance between the primary vertex and the track trajectory) of all tracks associated with a calorimeter jet [28].

- Secondary Vertex Tagging (SVT) - Based on the reconstruction of displaced secondary vertices [29].

- Counting Signed Impact Parameter (CSIP) - Based on the impact parameter significance $\left(I P / \sigma_{I P}\right)$ of the tracks in the jet cone, it requires a certain number of tracks to have a significance greater than 2 or 3 [30].

Variables from each of these algorithms are used to train the b-jet neural network. The most important variables are found to be [31]:

- JLIP

- Probability that the jet originated from the primary vertex,

- SVT

- Secondary vertex (SVX) with largest decay length significance,

- Number of tracks used for SVX reconstruction,

- Invariant mass of tracks in SVX calculation,

- Number of SVX's in jets,

$-\chi_{d o f}^{2}$ of each SVX.

- CSIP

- Weighted combination of tracks' IP significance. 
The b-jet neural network output gives a much improved signal to fake rate ratio over any of the individual algorithms. The b-jet efficiency against fake rate is shown in Figure 4.3 [31]. The efficiency is determined in a MC $Z \rightarrow b \bar{b}$ sample. The fake rate is assessed in a MC $Z \rightarrow q \bar{q}$ sample (where $q$ is a non-b-quark) and is the number of tagged jets compared to the total number of jets in the sample.

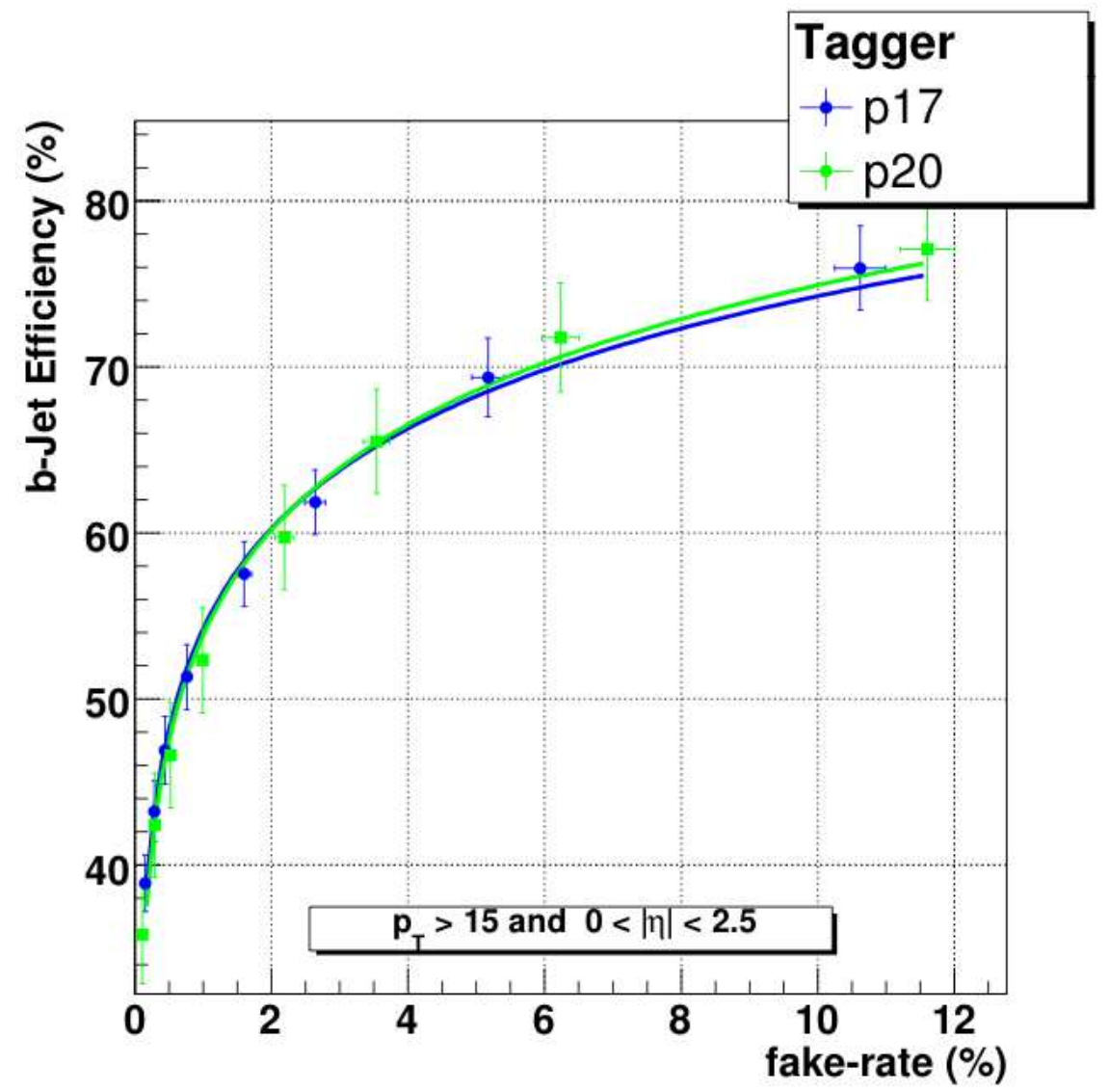

Figure 4.3: Signal efficiency and fake rate for the b-tagging neural network [31]. p17 and p20 are the reconstruction software versions for RunIIa data and RunIIb data respectively.

Various b-tagging operating points are defined by different requirements on the b-jet neural network output, where higher values of the output correspond to a higher purity of b-jets in the sample, but at a reduced efficiency. The operating points that are used in this analysis are L3 (NN output $>0.25)$ and VeryTight (NN output $>0.85)$. The L3 operating point has an efficiency for b-jets of $71.1 \%$ 
and a fake rate of around $7 \%$. The VeryTight operating point has an efficiency for b-jets of $44.1 \%$, however, the fake rate is reduced to around $0.3 \%$.

\subsubsection{Multi-jet Triggers}

Triggering on hadronic tau decays at a hadron collider is very challenging, due to the vast amount of hadronic activity in the detector during $p \bar{p}$ collisions. It is impossible to set up a trigger based on a single hadronic tau object in an event at a realistic trigger rate, without a very high $p_{T}$ threshold on the tau candidate, which would result in a substantial loss of signal acceptance for this analysis. It is therefore necessary to use triggers based upon each of the objects found in the associated Higgs candidate event and its topology to reduce the rate.

This analysis uses a suite of triggers based on jets plus missing transverse energy since the Higgs signal contains two jets and a neutrino. The L3 trigger always counts a hadronic tau decay as a jet. A different set of triggers is used for the RunIIa and RunIIb data periods and the triggers used in this analysis along with the luminosity gathered during that period are listed in Table 4.1. "Delivered" indicates the integrated luminosity of data that was delivered to D $\varnothing$ by the Tevatron, "Recorded" is the integrated luminosity of data that D $\varnothing$ wrote to tape and "Good" is the integrated luminosity of recorded data that passed data quality requirements.

RunIIa trigger terms At Level 1 (L1) different requirements are made of the calorimeter towers using terms of the form: CJT (X, Y, Z), where X calorimeter towers are required above an $E_{T}$ threshold Y GeV for $\left|\eta_{d}\right|<$ Z. For the MHT30_3CJT5 trigger the L1 term is CJT $(3,5,3.2)$, whereas the JT1_ACO_MHT_HT trigger additionally accepts two other terms: $\operatorname{CJT}(3,4,2.4)$ and $\operatorname{CJT}(1,7,1.8)$.

At Level $2(\mathrm{~L} 2)$ the vector sum of $p_{T}$ from all jets $\left(H_{\mathrm{T}}\right)$ is required to be above 


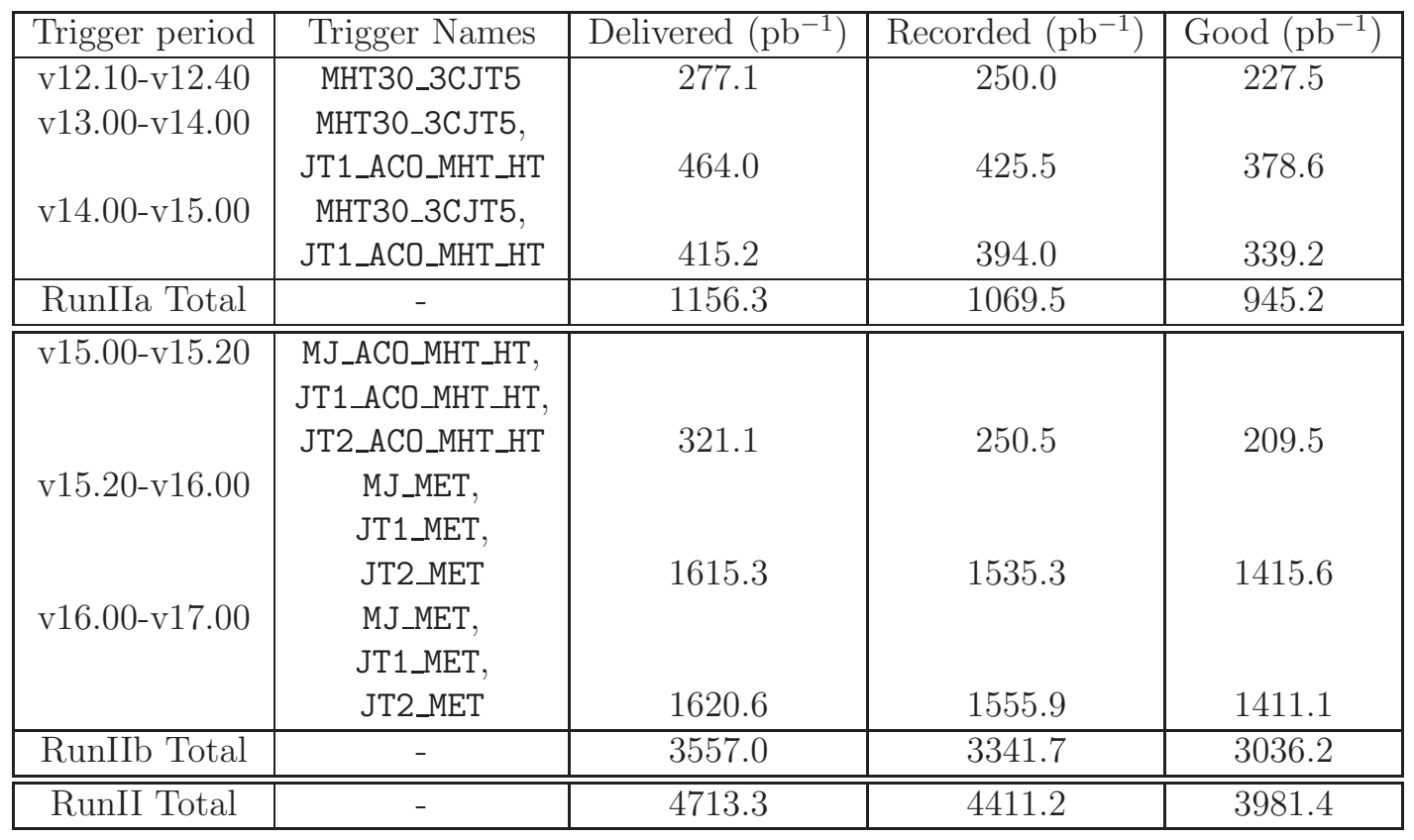

Table 4.1: Triggers used and luminosity accrued per trigger period.

a threshold of $20 \mathrm{GeV}$ and the angle $\Delta \phi$ between the leading two L2 jets in the event is required to be less than $168.75^{\circ}$ for the JT1_ACO_MHT_HT trigger. This is to remove back-to-back di-jet events.

At Level 3 (L3) the $H_{\mathrm{T}}$ cut is raised to $30 \mathrm{GeV}$, additionally the scalar sum of $p_{T}$ from jets $\left(H_{T}\right)$ is required to be greater than $50 \mathrm{GeV}$ for the JT1_ACO_MHT_HT trigger. The $\Delta \phi$ between the leading two L3 jets in the event is required to be less than $170^{\circ}$ and the minimum $\Delta \phi$ angle between any jet and the $H_{\mathrm{T}}$ is required to be greater than $25^{\circ}$ for the JT1_ACO_MHT_HT trigger.

RunIIb trigger terms The triggers used in RunIIb only differ from RunIIa at L3. However, there are more L1 terms to consider using a calorimeter "sliding windows" algorithm [32]. At L1 the following conditions must be satisfied:

- $\operatorname{CSWMET}(24)$ and $\operatorname{CSWJT}(1,30,3.2)$ (for the L1 mono jet term) or

- $\operatorname{CSWMET}(24)$ and $\operatorname{CSWJT}(1,20,2.4)$ and $\operatorname{CSWJT}(2,8,2.4)$ and ACOKILL (for the L1 dijet term) or 
- $\operatorname{CSWJT}(1,30,2.4)$ and $\operatorname{CSWJT}(2,15,2.4)$ and $\operatorname{CSWJT}(3,8,3.2)$ (for the L1 multijet term),

where at L1 an event must have calorimeter $\mathscr{E}_{\mathrm{T}}>\mathrm{X}$ in the calorimeter to pass the $\operatorname{CSWMET}(\mathrm{X})$ term. An event passes the $\operatorname{CSWJT}(\mathrm{X}, \mathrm{Y}, \mathrm{Z})$ term if it has $\mathrm{X}$ L1 jets with $E_{T}>\mathrm{Y} \mathrm{GeV}$ for $\left|\eta_{d}\right|<\mathrm{Z}$. An event passes the ACOKILL term if all pairs of jets with $8 \mathrm{GeV}<p_{T}<20 \mathrm{GeV}$ are not back-to-back in $\phi$.

At L2 the following requirements must be satisfied for each of the triggers:

- At least one L2 jet with $E_{T}>20 \mathrm{GeV}$ from $\left|\eta_{d}\right|<2.4$,

- $H_{\mathrm{T}}>20 \mathrm{GeV}$, using L2 jets with $E_{T}>10 \mathrm{GeV}$,

- $H_{T}>35 \mathrm{GeV}$, using L2 jets with $E_{T}>6 \mathrm{GeV}$ from $\left|\eta_{d}\right|<2.6$,

- $\Delta \phi$ between two leading L2 jets less than $168.75^{\circ}$

At L3 the requirements differ for the v15.00-v15.20 and v15.20-v17.00 trigger periods, the v15.00-v15.20 period triggers require:

- At least one L3 jet with $E_{T}>9 \mathrm{GeV}$,

- $H_{T}>50 \mathrm{GeV}$,

- $H_{\mathrm{T}}>30 \mathrm{GeV}$,

- $\Delta \phi$ between two leading L3 jets less than $170^{\circ}$,

- The minimum $\Delta \phi$ between all jets and $H_{\mathrm{T}}>25^{\circ}$.

The v15.20-v17.00 period triggers require:-

- At least two L3 jets with $E_{T}>9 \mathrm{GeV}$,

- $\mathbb{E}_{\mathrm{T}}>30 \mathrm{GeV}$, 
- $H_{\mathrm{T}}>25 \mathrm{GeV}$,

- $\Delta \phi$ between two leading L3 jets less than $170^{\circ}$,

- The minimum $\Delta \phi$ between all jets and $H_{\mathrm{T}}>25^{\circ}$.

\subsection{Simulation}

\subsubsection{Generation of Events}

To understand the response of the detector to physics processes, it is necessary to simulate the event kinematics with Monte Carlo (MC) event generators and then simulate the effects of the detector. These simulations adopt a numerical approach to solve a physical problem. The production of $\mathrm{MC}$ events that model physics processes as observed in the detector is a multi-stage procedure:

\section{- The Event}

- Parton interactions - The hard scatter is the first step of generation and simulates the event kinematics using the associated matrix elements.

- Parton Distribution Functions (PDFs) - The distribution of partons within the colliding hadrons are described by PDFs. The PDFs $[33,34]$ have been measured by the ZEUS and H1 experiments at the HERA accelerator at the DESY laboratory in $e p$ scattering as well as several fixed target experiments. The CTEQ6L1 leading order distribution function set [33] is used in the generation of MC samples used in this analysis.

- Parton Showering - This stage simulates the parton showering that occurs from the incoming and outgoing partons of the hard scatter. 
The process assumes a sequence of gluon splittings or gluon radiations from the partons involved.

- Hadronisation - This stage simulates the formation of hadrons from partons and their subsequent decay into stable particles.

\section{- The Detector}

- Detector effects - GEANT [35] is used to simulate the way in which a generated particle passes through the detector. It simulates the detector geometry and material, the interaction of particles with the material, as well as the trajectories and showering effects.

- Additional collisions - It is possible to have multiple $p \bar{p}$ collisions in a beam crossing. To simulate this effect, real events from the detector are overlaid on top of the MC events. These zero bias (ZB) events are taken using a dedicated ZB trigger and are overlaid on the MC events according to the instantaneous luminosity profile of the data.

A variety of different $\mathrm{MC}$ event generators are used in this analysis to simulate different physical processes:

PYTHIA As a leading order matrix element generator PYTHIA [36] is used to simulate hard scattering diagrams and to model parton showering and hadronisation. PYTHIA v6.413 is used in this analysis for the modelling of Higgs and diboson events.

ALPGEN The simulation of $W / Z$ production in combination with parton radiation and $t \bar{t}$ events is performed by ALPGEN v2.11 [37] as it allows the generation of vector bosons with hard parton radiation. It is interfaced with PYTHIA for the parton showering as ALPGEN only simulates the hard scatter. 


\begin{tabular}{|lc|lc|}
\hline Sample & k-factor $\times$ HF-factor & Sample & k-factor $\times$ HF-factor \\
\hline $\mathrm{W}+$ light flavors & 1.3 & $\mathrm{Z}+$ light flavors & 1.3 \\
$\mathrm{~W}+\mathrm{c}$ & $1.3 \times 1.47$ & $\mathrm{Z}+\mathrm{c}$ & $1.3 \times 1.67$ \\
$\mathrm{~W}+\mathrm{b}$ & $1.3 \times 1.47$ & $\mathrm{Z}+\mathrm{b}$ & $1.3 \times 1.52$ \\
\hline$t \bar{t} \rightarrow$ any decay & 1.43 & $\mathrm{WW}\left(\rightarrow l \nu l^{\prime} \nu\right)$ & 1.0 \\
single top $\rightarrow l \nu b($ s-channel $)$ & 0.99 & $\mathrm{WZ}\left(\rightarrow l \nu l^{\prime} l^{\prime}\right)$ & 1.06 \\
single top $\rightarrow l \nu b($ t-channel $)$ & 0.99 & $\mathrm{ZZ}\left(\rightarrow l l l^{\prime} l^{\prime}\right)$ & 1.03 \\
\hline
\end{tabular}

Table 4.2: $k$-factors [39] and heavy flavour scale factors [40] used for MC processes.

COMPHEP The single top process is simulated using COMPHEP [38], which is also interfaced with PYTHIA for the parton showering.

\section{Normalising the MC simulation}

The MC is normalised using the integrated luminosity $(L)$ of the data set, the number of simulated events $\left(N_{M C}\right)$ and the cross-section for the process $(\sigma)$ such that the weight for each MC event $w_{M C}$ is given by

$$
w_{M C}=\frac{\sigma L}{N_{M C}}
$$

The number of simulated events is considered after removal of events where the zero bias overlay came from events in a bad run or runs with a bad LBN (see Section 3.2.8).

The cross-sections for the ALPGEN processes are calculated at leading order. The next-to-leading order corrections are taken into account by the application of a $k$-factor [39]. An additional heavy flavour scale factor is applied to the processes with additional $b$ and $c$ quarks in the final state to correctly model the heavy flavour fraction. It is taken from MCFM [40]. Table 4.2 shows the $k$-factors and heavy flavour scale factors that are applied to the different simulated MC processes.

A list of the signal and background MC samples used in this analysis, along with the associated cross-sections, is shown in Tables 4.3 and 4.4, respectively. 
Different MC samples are used for the different data taking periods to reflect detector changes during that time. RunIIa uses the p17 reconstruction software version and RunIIb uses the p20 reconstruction software version.

\begin{tabular}{|c|c|}
\hline Simulated Process & Cross-section - $\sigma(\mathrm{pb})$ \\
\hline$Z H \rightarrow \ell \ell b b, m_{H}=100 \mathrm{GeV}$ & 0.01368 \\
$W H \rightarrow \ell \nu b \bar{b}, m_{H}=100 \mathrm{GeV}$ & 0.07528 \\
\hline$Z H \rightarrow \ell \ell b b, m_{H}=105 \mathrm{GeV}$ & 0.01149 \\
$W H \rightarrow \ell \nu b \bar{b}, m_{H}=105 \mathrm{GeV}$ & 0.06271 \\
\hline$Z H \rightarrow \ell \ell b b, m_{H}=110 \mathrm{GeV}$ & 0.00959 \\
$W H \rightarrow \ell \nu b \bar{b}, m_{H}=110 \mathrm{GeV}$ & 0.05183 \\
\hline$Z H \rightarrow \ell \ell b b, m_{H}=115 \mathrm{GeV}$ & 0.00789 \\
$W H \rightarrow \ell \nu b \bar{b}, m_{H}=115 \mathrm{GeV}$ & 0.04225 \\
\hline$Z H \rightarrow \ell \ell b b, m_{H}=120 \mathrm{GeV}$ & 0.00635 \\
$W H \rightarrow \ell \nu b \bar{b}, m_{H}=120 \mathrm{GeV}$ & 0.03368 \\
\hline$Z H \rightarrow \ell \ell b b, m_{H}=125 \mathrm{GeV}$ & 0.00496 \\
$W H \rightarrow \ell \nu b \bar{b}, m_{H}=125 \mathrm{GeV}$ & 0.02611 \\
\hline$Z H \rightarrow \ell \ell b b, m_{H}=130 \mathrm{GeV}$ & 0.00374 \\
$W H \rightarrow \ell \nu b \bar{b}, m_{H}=130 \mathrm{GeV}$ & 0.01956 \\
\hline$Z H \rightarrow \ell \ell b b, m_{H}=135 \mathrm{GeV}$ & 0.00271 \\
$W H \rightarrow \ell \nu b \bar{b}, m_{H}=135 \mathrm{GeV}$ & 0.01404 \\
\hline$Z H \rightarrow \ell \ell b b, m_{H}=140 \mathrm{GeV}$ & 0.00188 \\
$W H \rightarrow \ell \nu b \bar{b}, m_{H}=140 \mathrm{GeV}$ & 0.00964 \\
\hline$Z H \rightarrow \ell \ell b b, m_{H}=145 \mathrm{GeV}$ & 0.00123 \\
$W H \rightarrow \ell \nu b \bar{b}, m_{H}=145 \mathrm{GeV}$ & 0.00626 \\
\hline$Z H \rightarrow \ell \ell b b, m_{H}=150 \mathrm{GeV}$ & 0.00074 \\
$W H \rightarrow \ell \nu b \bar{b}, m_{H}=150 \mathrm{GeV}$ & 0.00377 \\
\hline
\end{tabular}

Table 4.3: Signal MC samples with cross sections in pb for various Higgs boson masses [41].

The analysis includes $W H \rightarrow \ell \nu b \bar{b}$ and $Z H \rightarrow \ell \bar{\ell} b \bar{b}$ samples which are combined together to form the signal. Events from the $Z H \rightarrow \ell \bar{\ell} b \bar{b}$ sample where one of the leptons escapes detection can add valuable sensitivity to the overall signal in this channel. In subsequent distributions the signal is the sum of these two physical processes scaled to the appropriate cross-sections as indicated in Table 4.3. 


\begin{tabular}{|c|c|}
\hline Simulated Process & Cross-section $-\sigma(\mathrm{pb})$ \\
\hline$W \rightarrow \ell \nu$ & 6200 \\
$W+c \bar{c} \rightarrow \ell \nu+c \bar{c}$ & 45.2 \\
$W+b \bar{b} \rightarrow \ell \nu+b \bar{b}$ & 15.8 \\
\hline$Z \rightarrow \ell \ell$ & 1725.8 \\
$Z \rightarrow \nu \bar{\nu}$ & 891.25 \\
$Z+c \bar{c} \rightarrow \ell \bar{\ell}+c \bar{c}$ & 22.1 \\
$Z+c \bar{c} \rightarrow \nu \bar{\nu}+c \bar{c}$ & 9.56 \\
$Z+b \bar{b} \rightarrow \ell \bar{\ell}+b \bar{b}$ & 4.44 \\
$Z+b \bar{b} \rightarrow \nu \bar{\nu}+b \bar{b}$ & 4.37 \\
\hline$W \rightarrow$ all final states & 3.25 \\
$W W \rightarrow$ all final states & 11.6 \\
$Z Z \rightarrow$ all final states & 1.33 \\
\hline$t b \rightarrow \ell \nu b b$ & 0.339 \\
$t q b \rightarrow \ell \nu b q \bar{b}$ & 0.736 \\
$t \bar{t} \rightarrow b \bar{b}$ & 2.41 \\
$t \bar{t} \rightarrow \ell \nu b \bar{b}$ & 2.41 \\
$t \bar{t} \rightarrow \ell \bar{\ell} \nu \bar{\nu} b \bar{b}$ & 0.61 \\
\hline
\end{tabular}

Table 4.4: Background MC samples with cross sections [42] (without $k$-factors). Events are simulated exclusively for additional light parton multiplicities (except diboson and single top events) and are summed in this table.

\subsubsection{Correcting the MC Simulation}

Although the MC has been passed through a full detector simulation there are still residual effects that have to be taken into account. These effects range from corrections, to take into account differences between data and $\mathrm{MC}$, to simulating the effect of the trigger in $\mathrm{MC}$. The different corrections applied to the $\mathrm{MC}$ are described in the rest of this chapter.

\section{Tau Identification efficiency}

A correction factor is derived in a dedicated $Z \rightarrow \tau \tau$ sample for the efficiency of the hadronic tau neural network cut as a function of the neural network output for different tau types. Data and $Z \rightarrow \tau \tau$ MC are compared, and a probability distribution function, "PDF" is defined. For MC this PDF is the fraction of the total number of events in a particular neural network output bin. For data the 
PDF is the number of data events in a particular neural network output bin after all backgrounds are subtracted (not $Z \rightarrow \tau \tau$ ). This allows a direct comparison between $Z \rightarrow \tau \tau$ events in data and in MC, this distribution is shown in the left panel of Figure 4.4. A correction to the tau neural network distribution is then derived bin-by-bin, and applied as a weight to the MC. The MC correction factor as a function of tau neural network output in shown in the right panel of Figure 4.4. The effect is found to be small when a high purity tau sample is required. However, this becomes more significant as more background leaks into the sample, as can be seen from the right panel of Figure 4.4 [43]. The magnitude of this correction for the tau neural network cuts used in this analysis is around $2 \%$.
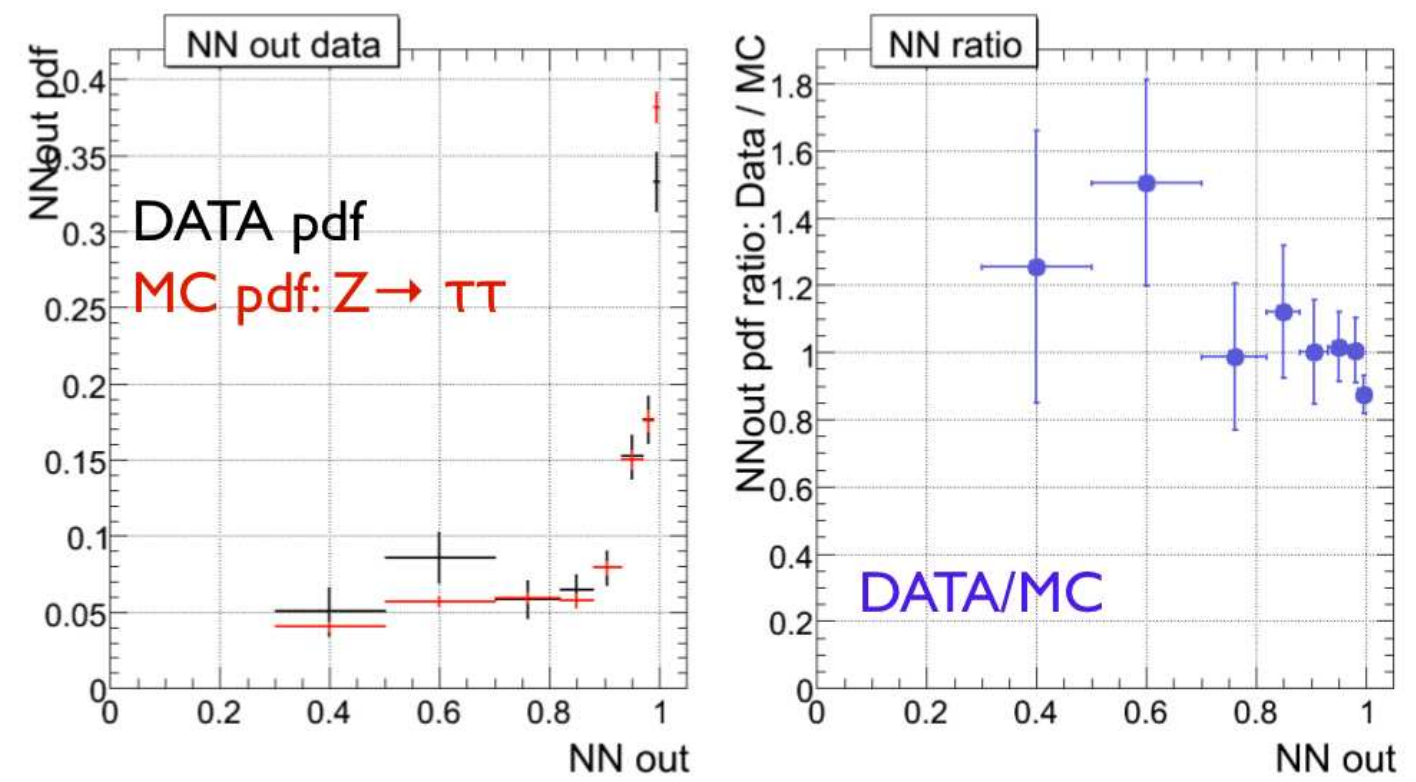

Figure 4.4: Data and MC $\tau$ neural network output PDFs vs $\tau$ neural network output (left panel) and $\tau$ efficiency correction vs $\tau$ neural network output (right panel) for type 2 taus [43].

An additional correction is applied to the MC to correctly simulate the efficiency for finding a tau track in the data. The method used to derive this correction is identical to that used in muon identification [21]. This correction varies depending upon the quality of track that is selected and its transverse 
momentum, and is typically around $2 \%$ for the tracks selected in this analysis.

\section{Jet Smearing, Shifting and Removal}

To model the differences between data and MC in jet energy, jet energy resolution and jet identification efficiency, a procedure known as jet smearing, shifting and removal (JSSR) is applied [44]. A study was undertaken using data and simulated photon plus jet events. The transverse momentum imbalance $\Delta S$ is contructed where:

$$
\Delta S=\frac{p_{T}^{j e t}-p_{T}^{\gamma}}{p_{T}^{\gamma}} .
$$

A Gaussian function is then fitted to the $\Delta S$ distributions in different bins of the photon transverse momentum $p_{T}^{\gamma}$. The mean of the Gaussian gives the jet energy scale and the width gives the jet energy resolution. The MC can be corrected by shifting the jet energy to the mean value of the Gaussian and by smearing the energy resolution to match that of the Gaussian.

The jet identification efficiency is corrected for in the MC by constructing turn on curves using error functions formed by the difference $(D)$ between the gaussian means in data and MC. The removal procedure removes those jets in MC whose transverse momentum lies below the plateau of the error function defined by the difference $(D)$.

\section{Reweighting of $W / Z$ transverse momentum}

The MC events generated using PYTHIA and ALPGEN do not correctly reproduce the vector boson transverse momentum $\left(p_{T}^{W / Z}\right)$ spectrum. The transverse momentum of the $Z$ bosons is unfolded from a $Z \rightarrow$ ee measurement and a parameterisation derived to fit the reweighting factor in bins of $p_{T}^{Z}[45]$. For the $W$ transverse momentum reweighting a direct measurement of $p_{T}^{W}$ does not exist due 
to the neutrino in the final state. Instead the ratio of differential cross-sections $\left(d \sigma / d p_{T}\right)$ for $W$ and $Z$ events is used as the reweighting variable. The reweighting of the $p_{T}^{W}$ is done in exclusive jet multiplicity bins [46].

\section{Instantaneous Luminosity Reweighting}

To ensure that the instantaneous luminosity profile of the MC matches that of the data events, a reweighting is performed in the MC. This correction is more significant in the RunIIb portion of the data as the instantaneous luminosity is spread over a wider range of values (arising from a higher peak value at the beginning of stores).

\section{Jet and Track scale factors}

As the tracking efficiency for MC is higher than that in data it is necessary to apply scale factors to the MC to ensure agreement with the data. This effect is important when applying cuts on jet-track variables as described below.

Vertex Confirmation To account for differences in the modelling of track variables between data and MC after vertex confirmation (see Section 4.1.4), a series of scale factors are applied. These are derived in three independent samples: $\gamma+$ jet, $Z(\rightarrow e e / \mu \mu)+$ jet and di-jet events. The scale factors are derived as a function of the $z$ position of the primary vertex, $\eta_{d}($ jet $)$ and $p_{T}($ jet $)$ to ensure data - MC agreement [47].

Taggability scale factors In a similar way to the vertex confirmation scale factors, the difference in tracking efficiency between data and MC necessitate taggability scale factors. These scale factors are studied in $W / Z+$ jets samples and as with the vertex confirmation scale factors, they depend on the $z$ position 
of the primary vertex, $\eta_{d}($ jet $)$ and $p_{T}$ (jet). These scale factors are parameterised for each jet multiplicity bin in MC events [48].

b-tagging scale factors As the b-tagging algorithm makes use of many jet and track variables, a higher efficiency is found in MC than in data when direct tagging is applied. As different b-tag operating points represent a different cut on the b-tagging neural network output, different scale factors are derived for each operating point.

\section{Trigger parameterisation}

The modelling of a suite of three stage triggers with multiple objects in the trigger terms is complicated and is fully described in a study that is performed independent of this analysis [49]. However, the important steps are summarised here including some of the changes that are made to the trigger parameterisation to account for hadronic tau decays, which pass the requirements of these multi-jet triggers.

To measure the probability that an event will be selected by the suite of triggers $(P(L 1, L 2, L 3))$, measurements of the efficiency of each level of the trigger are considered and then the final probability is calculated as follows:

$$
P(L 1, L 2, L 3)=P(L 1) \times P(L 2 \mid L 1) \times P(L 3 \mid L 1, L 2),
$$

where $P(L 3 \mid L 1, L 2)$ is the conditional probability that an event passes the L3 requirements having already passed L1 and L2.

The modelling of the L1 terms is performed by constructing a turn-on curve to represent the acceptance of a jet of a given $p_{T}$ passing a particular $p_{T}$ requirement online at the trigger level. For the L1 jet trigger terms, the number of calorimeter trigger towers that have a transverse momentum above the required threshold, 
are considered to have been fired by a jet. A turn on curve in jet transverse momentum is then constructed. For the $\mathrm{L} 1 \boldsymbol{F}_{\mathrm{T}}$ terms, first a shifting and smearing of the L1 $\mathbb{E}_{\mathrm{T}}$ is applied to bring the MC simulation in agreement with the data, then a turn-on curve is constructed as with the jet terms. An example of the turn on curves for the RunIIb L1 jet terms is shown in Figure 4.5 [49], where "pTcorr" is the corrected MC jet $p_{T}$ in $\mathrm{GeV}$.
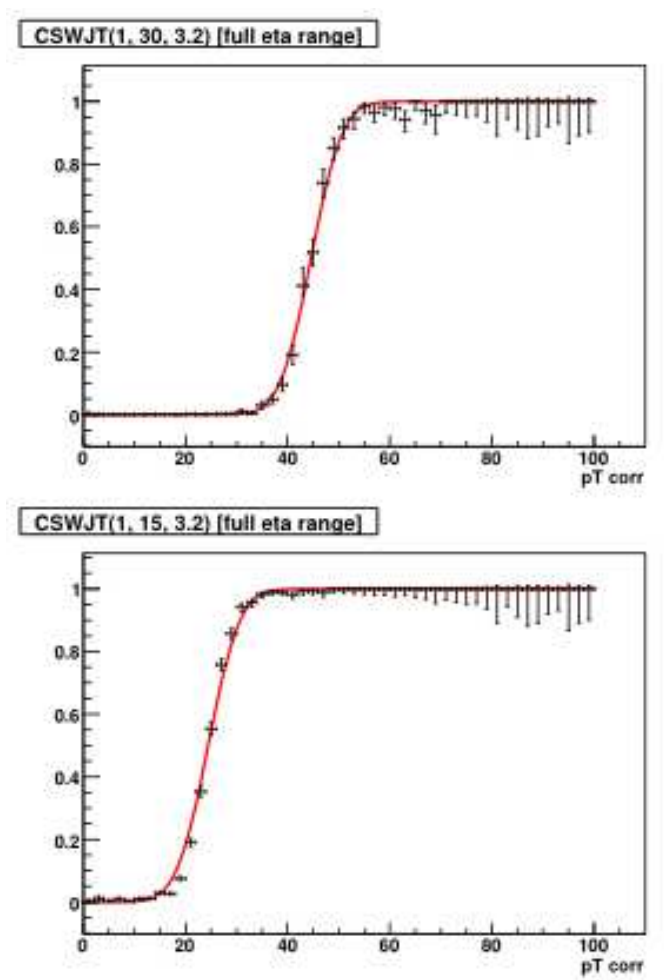
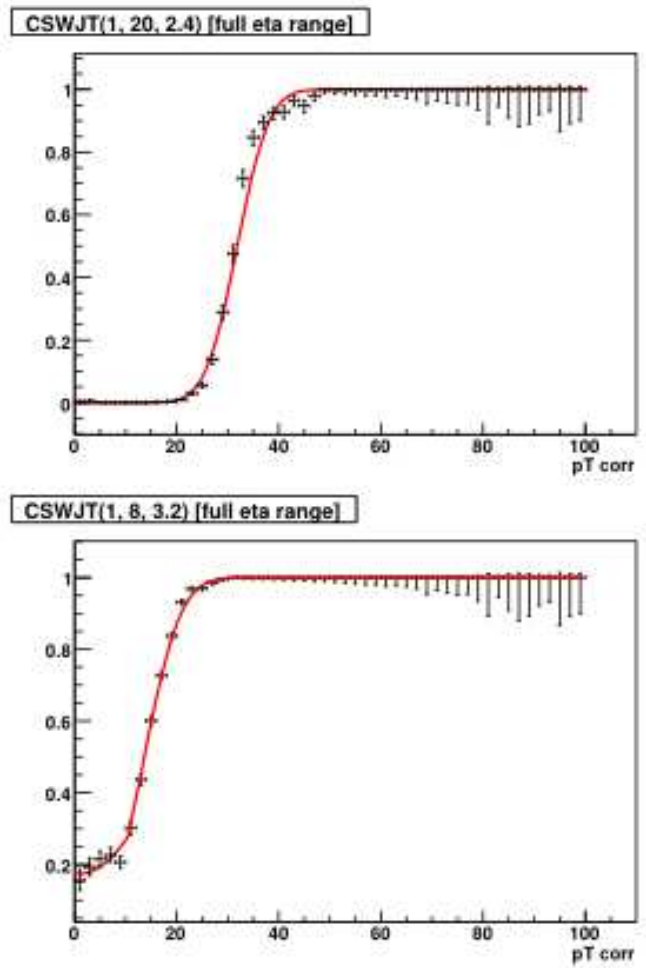

Figure 4.5: L1 jet turn-on curves for RunIIb triggers as a function of the corrected MC jet $p_{T}($ in $\mathrm{GeV})[49]$.

The turn-on curves are parameterised by error functions which plateau once the jet $p_{T}$ is sufficiently above the threshold of the trigger term. The threshold for the trigger is typically found near the beginning of the turn on curve.

As the L2 rejection is very small it is assumed to be $100 \%$ efficient with respect to L1, so only the L3 terms are parameterised. The L3 parameterisation calculates the probability that an offline jet is reconstructed with the same energy 
as the corresponding L3 jet. This allows each of the L3 variables such as $H_{\mathrm{T}}$ and $H_{T}$ to be calculated and parameterised [49]. 


\section{Chapter 5}

\section{Modeling the $\tau \nu b \bar{b}$ Final State}

\subsection{Event Selection}

\subsubsection{Data Samples}

The data used in this analysis comprise approximately $4 \mathrm{fb}^{-1}$ of RunII(a+b) luminosity collected using the $\mathrm{D} \varnothing$ Detector. The data is taken from the New Phenomena skim which requires that the data were collected using one of the triggers requiring a jets plus missing energy topology and $H_{\mathrm{T}}>15 \mathrm{GeV}$. Data quality requirements are applied to the data before a pre-selection is made.

\subsubsection{Object Selection}

\section{Tau Leptons}

A tau candidate is required in the event. No specific requirements are made of other tau candidates in the event, since it is found that there are very few events which contain more than one "loose" quality tau, defined as having a hadronic tau neural network output $\left(\tau_{n n_{h}}\right)>0.3$. No events contain a second "tight" 
quality tau, defined as having $\tau_{n n_{h}}>0.9$. It was found in previous studies that the sensitivity achieved in type 3 tau candidate events is significantly worse than that in the type 1 and type 2 candidate events. Additionally, the modelling of the background from jet fakes to type 3 tau candidate events proves very difficult, so this analysis only considers type 1 and type 2 (single track) candidate events. Kinematic and neural network selections for these taus are as follows:

- Type 1 Taus:

$$
\begin{aligned}
& p_{T}>12 \mathrm{GeV} \\
& p_{T_{t r k}}>7 \mathrm{GeV} \\
& \tau_{n n_{h}}>0.3 \\
& \text { Tau }\left|\eta_{d}\right|<2.0
\end{aligned}
$$

- Type 2 Taus:

$$
\begin{aligned}
& p_{T}>10 \mathrm{GeV} \\
& p_{T_{t r k}}>5 \mathrm{GeV} \\
& \tau_{n n_{h}}>0.3 \\
& \text { Tau }\left|\eta_{d}\right|<2.0
\end{aligned}
$$

The transverse momentum cuts on the type 2 taus are slightly lower than for type 1 taus because they benefit from the additional requirement of an EM sub-cluster which helps to reduce the background. The reason for requiring only one "loose" tau rather than a "tight" tau at this stage of the analysis is so that a multi-jet fake estimate can be made. Tau candidates with a quality between "loose" and "tight" are used for this estimate, and the quality criteria are raised in the analysis selection sample at a later stage. 


\section{Jets}

Reconstructed jet energies are corrected based on the expected calorimeter response, energy lost due to showering out of the jet cone, and energy deposited in the jet cone not associated with the jet (see Section 4.1.2). The jet selection requires that there are two or three jets of $p_{T}>20 \mathrm{GeV}$ and detector $\left|\eta_{d}\right|<2.5$ in the event. These jets must be separated from the tau candidate such that $\Delta R(\tau, j e t)>0.5$. It is required that at least two jets are vertex confirmed for RunIIb and that they are taggable. A looser jet selection is made to select jets which are used to correct the $E_{\mathrm{T}}$ in the event. Any jets in the event with $p_{T}>$ $9 \mathrm{GeV}$ and $\left|\eta_{d}\right|<2.5$ are considered for this selection.

\section{Electrons and Muons}

The purpose of the electron and muon selection in the analysis is twofold. Firstly, leptons that are selected in other Higgs boson searches must be removed from the analysis sample so the analyses remain statistically independent. Secondly, electrons and muons must be selected to correct the missing transverse energy in the event.

It is possible for this analysis to gain sensitivity from electrons that are reconstructed as tau candidates, so it is important that the electrons which are selected in other Higgs analyses are removed. For muons however, very little sensitivity can be gained from muons faking tau candidates, so a simple veto on events containing low quality isolated muons is employed.

Electron events are vetoed from the analysis sample if they fulfill the following criteria (variables defined in Section 4.1.1):

- Event contains one electron either in the $\mathrm{CC}$ or EC region of the detector,

- Isolation $>0.15$, 
- EM fraction > 0.9,

- H-matrix > 50,

- Likelihood > 0.85,

- $p_{T_{t r k}}>5 \mathrm{GeV}$,

- $p_{T}>15 \mathrm{GeV}$,

- $\mathbb{F}_{\mathrm{T}}>20 \mathrm{GeV}$ if $\left|\eta_{d}\right|<1.1$,

- $H_{\mathrm{T}}>25 \mathrm{GeV}$ if $1.5<\left|\eta_{d}\right|<2.5$,

- $m_{T}(\mathrm{~W})>\left(40-0.5 \times \not_{\mathrm{T}}\right) \mathrm{GeV}$,

- $p_{T}($ jet1 $)>25 \mathrm{GeV}$,

- Two or more vertex confirmed (for RunIIb) jets with $p_{T}>20 \mathrm{GeV}$ with $\left|\eta_{d}\right|<2.5$,

- $H_{T}>60 \mathrm{GeV}$.

Muon events are vetoed from the analysis sample if they fulfill the following criteria (variables defined in Section 4.1.1):

- $p_{T}>15 \mathrm{GeV}$,

- "loose" quality muon matched to a track of "medium" quality,

- DCA of tracks with SMT hits $<0.02$,

- DCA of tracks without SMT hits $<0.2$,

- Scaled calorimeter isolation $<0.08$,

- Scaled track isolation $<0.06$, 
- $\chi^{2} /$ dof from track-muon matching $<4$,

- $\Delta R$ from jets $>0.5$.

Having removed those leptons selected in the $W H \rightarrow(e / \mu) \nu b \bar{b}$ and $(W / Z) H \rightarrow$ $\tau \tau q \bar{q}$ analyses, a further selection on electrons and muons is made to correct the $E_{\mathrm{T}}$.

Electrons that are separated from jets and taus by $\Delta R>0.5$, and pass the following cuts:

- Isolation $<0.15$,

- EM fraction > 0.9,

- $p_{T}>5 \mathrm{GeV}$,

- $\left|\eta_{d}\right|<2.5$,

and muons that are separated from jets and taus by $\Delta R>0.5$, and are of "medium" quality are used in the $\mathbb{E}_{\mathrm{T}}$ re-computation.

\section{b-jets}

The neural network b-tagging algorithm is applied at the preselection stage and jets are tagged if they fulfill the VeryTight or L3 b-tagging criteria (see Section 4.1.4). This allows a b-tagging requirement to be imposed in the data after the backgrounds are studied in a high statistics sample as b-tagging significantly reduces the light quark events that are dominant in the preselected data sample.

\section{Missing Transverse Energy Selection}

The $E_{\mathrm{T}}$ is corrected for the presence of electrons, muons, taus and jets with the requirements specified above. To reduce some of the multi-jet background and to 
increase the fraction of $W$ events in the preselected sample, a $\mathbb{F}_{\mathrm{T}}>15 \mathrm{GeV}$ cut is imposed.

\subsubsection{Preselection Efficiency}

The efficiency of the preselection cuts on the data and the two signal samples is shown in Table 5.1 for a Higgs mass of $115 \mathrm{GeV}$.

The tau neural network cut removes much of the data as the number of reconstructed taus that are faked by jets is very high. The requirement that there exist two jets separated from the tau candidate is also a powerful cut in data, due to the large number of jets that are reconstructed as taus. The trigger requirement in data defaults to $100 \%$ efficient as the trigger is required in the skim before these selection cuts as reported in Section 5.1.1. These basic event selections have improved the signal to data (dominated by background at this early stage) ratio in the sample by over a factor of ten.

\subsection{Background Modelling}

To determine how well the background model fits the data, two distinct control regions are considered. These regions are designed to test the modelling of the electroweak and multi-jet backgrounds where the signal contribution is negligible. Many kinematic variables are studied when determining how well the background is modelled and a comprehensive set of distributions is shown in the Appendices. Kinematic distributions of particular interest are shown and discussed in the next two sections. 


\begin{tabular}{|c|c|c|c|}
\hline \multirow[t]{2}{*}{ Preselection Cut } & \multicolumn{3}{|c|}{$\operatorname{Data}\left(L=4 \mathrm{fb}^{-1}\right)$} \\
\hline & Events & Rel. $(\%)$ & Abs. $(\%)$ \\
\hline NP skim & 224986005 & & \\
\hline Duplicate events & 224986005 & 100.00 & 100.00 \\
\hline Bad runs \& LBNs & 193017786 & 85.79 & 85.79 \\
\hline Event quality & 175247246 & 90.79 & 77.89 \\
\hline Tau kinematic cuts & 25905665 & 14.78 & 11.51 \\
\hline Tau NN cut & 5498302 & 21.22 & 2.44 \\
\hline Trigger & 5498302 & 100.00 & 2.44 \\
\hline$N_{\text {jets }}(\Delta R(\tau$, jet $)>0.5) \leq 3$ & 1709718 & 31.10 & 0.76 \\
\hline$N_{\text {confirmed }_{\mathrm{j}} \text { ets }}(\Delta R(\tau$, jet $)>0.5) \geq 2$ & 1226744 & 71.75 & 0.55 \\
\hline$E_{\mathrm{T}}>15 \mathrm{GeV}$ & 1206341 & 98.34 & 0.54 \\
\hline & \multicolumn{3}{|c|}{$W H \rightarrow l \nu b b(l=e, \mu, \tau) \mathrm{MC}, m_{H}=115 \mathrm{GeV}$} \\
\hline NP skim & 447284 & & \\
\hline Duplicate events & 447284 & 100.00 & 100.00 \\
\hline Bad runs \& LBNs & 446403 & 99.80 & 99.80 \\
\hline Event quality & 432412 & 96.87 & 96.68 \\
\hline Tau kinematic cuts & 339630 & 78.54 & 75.93 \\
\hline Tau NN cut & 159485 & 46.96 & 35.66 \\
\hline$N_{\text {jets }}(\Delta \mathrm{R}(\tau$, jet $)>0.5) \leq 3$ & 95487 & 59.87 & 21.35 \\
\hline$N_{\text {confirmed jets }}(\Delta \mathrm{R}(\tau$, jet $)>0.5) \geq 2$ & 90992 & 95.29 & 20.34 \\
\hline Trigger Simulation & 32823 & 36.07 & 7.34 \\
\hline \multirow[t]{2}{*}{$\mathscr{E}_{\mathrm{T}}>15 \mathrm{GeV}$} & 32820 & 99.99 & 7.34 \\
\hline & \multicolumn{3}{|c|}{$Z H \rightarrow l l b b(l=e, \mu, \tau) \mathrm{MC}, m_{H}=115 \mathrm{GeV}$} \\
\hline NP skim & 479987 & & \\
\hline Duplicate events & 479987 & 100.00 & 100.00 \\
\hline Bad runs \& LBNs & 479085 & 99.81 & 99.81 \\
\hline Event quality & 464282 & 96.91 & 96.73 \\
\hline Tau kinematic cuts & 386382 & 83.22 & 80.50 \\
\hline Tau NN cut & 231819 & 60.00 & 48.30 \\
\hline$N_{\text {jets }}(\Delta \mathrm{R}(\tau$, jet $)>0.5) \leq 3$ & 145601 & 62.81 & 30.33 \\
\hline$N_{\text {confirmed jets }}(\Delta \mathrm{R}(\tau$, jet $)>0.5) \geq 2$ & 136206 & 93.55 & 28.38 \\
\hline Trigger Simulation & 18986 & 13.94 & 3.96 \\
\hline $\mathscr{E}_{\mathrm{T}}>15 \mathrm{GeV}$ & 18967 & 99.90 & 3.96 \\
\hline
\end{tabular}

Table 5.1: Preselection cut flow for data and signal MC events. 


\subsubsection{Multi-jet Control Region}

It is very difficult to model multi-jet events with simulated data. Due to the large cross-section it would take a huge number of simulated events to be able to accurately create kinematic distributions to the precision required in this analysis. In addition, the kinematics of multi-jet events are not well modelled by the MC generators. For this reason an estimate of the multi-jet background is made in a sideband region in data that is independent of the final analysis sample.

\section{Multi-jet Background}

The multi-jet (QCD) contribution is estimated by sampling a region that is dominated by fake taus from jets. The $\tau_{n n_{h}}$ cut is inverted to select a region orthogonal to the signal region. In particular, events are selected where the highest $\tau_{n n_{h}}$ tau lies in the range $0.3<\tau_{n n_{h}}<0.7$. The upper limit at 0.7 is chosen as for $\tau_{n n_{h}}>$ 0.7 a significant fraction of real taus would begin to enter the sample (this can be seen in Figure 4.1) and invalidate the assumption that this region is dominated by fake taus. Any MC generated backgrounds (such as W decays, the dominant physics background at this stage) that fall into this region are subtracted leaving a multi-jet enriched sample. The number of multi-jet events in the enriched sample, $N_{\text {rich }}^{Q C D}$, can be expressed as:

$$
N_{\text {rich }}^{Q C D}=N_{\text {rich }}-N_{\text {rich }}^{M C},
$$

where $N_{\text {rich }}$ is the number of events in the enriched region and $N_{\text {rich }}^{M C}$ is the number of $\mathrm{MC}$ events in the enriched region.

To normalise and determine how well the multi-jet background estimate performs, a control sample is considered where the contribution from MC processes is minimal. The additional requirements on top of the preselection to obtain this 
control sample are $\mathscr{E}_{\mathrm{T}_{\mathrm{SIG}}}<3.5$ and $\Delta \phi\left(\not_{\mathrm{T}}, \mathbb{E}_{\mathrm{T}}\right)>2.0$. The $\mathbb{E}_{\mathrm{T}_{\mathrm{SIG}}}$ is a useful variable for separating those events that have real $\mathscr{F}_{\mathrm{T}}$ from neutrinos and those that have fake $\mathscr{E}_{\mathrm{T}}$ from mis-measurements (see Section 4.1.3).

Initially a single normalisation factor independent of the kinematics is applied to the multi-jet events. This assumes the deficit between data and MC in this region is entirely made up of multi-jet background. The background is dominated by multi-jet events with very little electroweak background in this sample. The model is found to agree well for jet based variables (Figures 5.1(a,b)), but as expected does not perform as well for tau variables that are important in the tau neural network (Figures 5.1(c,d)). This is because a data sample with $\tau_{n n_{h}}>0.9$ is compared to a background model with $0.3<\tau_{n n_{h}}<0.7$. Figure 5.1 shows the agreement between data and background with a single multi-jet normalisation factor. Type 2 tau candidate events are shown here as the statistics are higher in that sample than that of type 1 tau candidate events.

\section{Reweighting the Multi-jet Estimation}

To improve the multi-jet modelling in tau variables such as $p_{T}(\tau)$ and $\eta_{d}(\tau)$ the multi-jet estimate is reweighted. The $p_{T}(\tau)$ reweighting $(\mathrm{RW})$ improves many tau variables as the $p_{T}(\tau)$ is directly correlated with them. The $\eta_{d}(\tau)$ reweighting is necessary as this distribution is an important discriminating variable in the hadronic tau neural network. Also for type 2 tau events it takes into account the fact that the sample is made up of both hadronic tau decays and electron fakes which could conceivably have different fake rates. The reweighting factors are derived in the type 2 tau sample due to the much higher statistics and applied to both type 1 and 2 tau candidate events as the kinematics are similar.

The $p_{T}(\tau)$ is reweighted using a 4 th order polynomial fit function and is shown in Figure 5.2(a). The $\eta_{d}(\tau)$ distribution is difficult to fit and so a bin-by-bin 


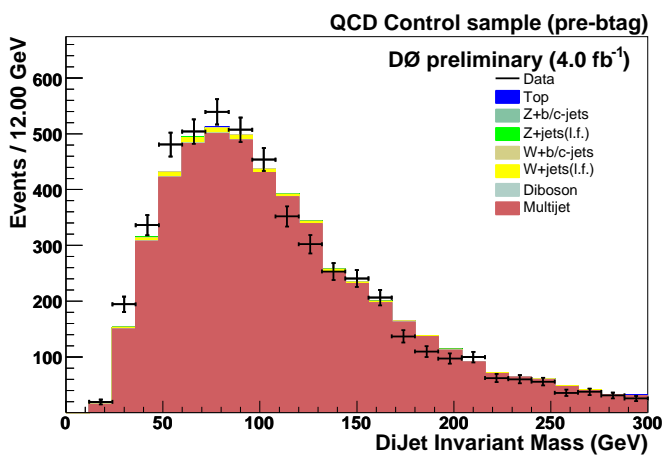

(a) Di-jet Mass

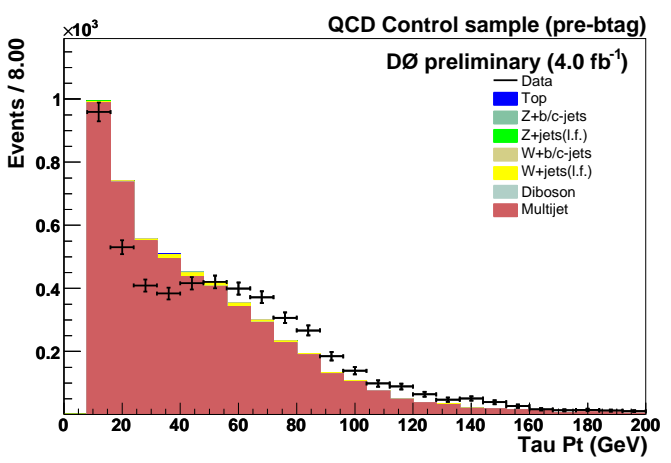

(c) $p_{T}(\tau)$

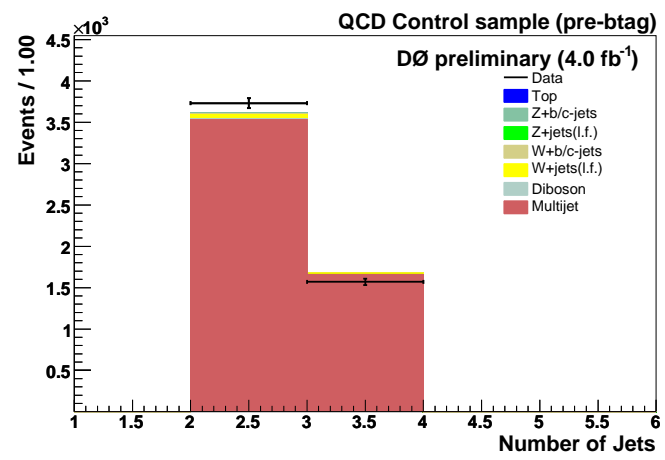

(b) Jet Multiplicity

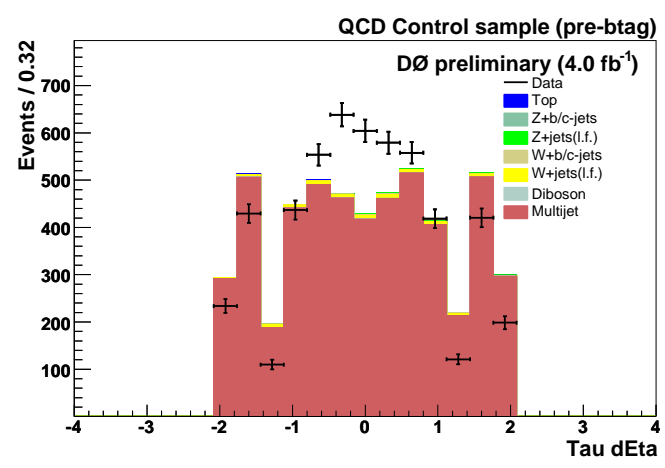

(d) $\eta_{d}(\tau)$

Figure 5.1: (a) Di-jet mass, (b) jet multiplicity, (c) $\tau$ transverse momentum and (d) $\tau$ detector eta in the multi-jet control sample with a single normalisation factor.

reweighting is applied using the histrogram shown in Figure 5.2(b).

Figure 5.3 shows the same kinematic distributions in the multi-jet control sample after this reweighting is applied. The $\eta_{d}(\tau)$ distribution is not perfect in the central region. This arises from the fact that two one-dimensional reweightings are applied instead of a single $2 \mathrm{D}$ reweighting in $p_{T}(\tau)$ and $\eta_{d}(\tau)$. Due to insufficient statistics a 2D reweighting was not deemed feasible in this control sample. The reweighting has improved many of the tau based distributions in this control sample, while preserving the already good agreement in jet variables. This normalisation scheme is applied to the analysis sample. Many more kinematic distributions from the multi-jet control sample are shown in Figure A.1, where the agreement between data and background is shown to be good. 


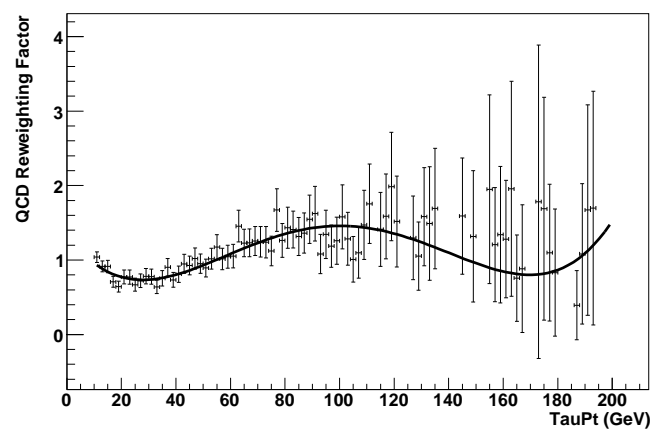

(a) $p_{T}(\tau)$ RW function

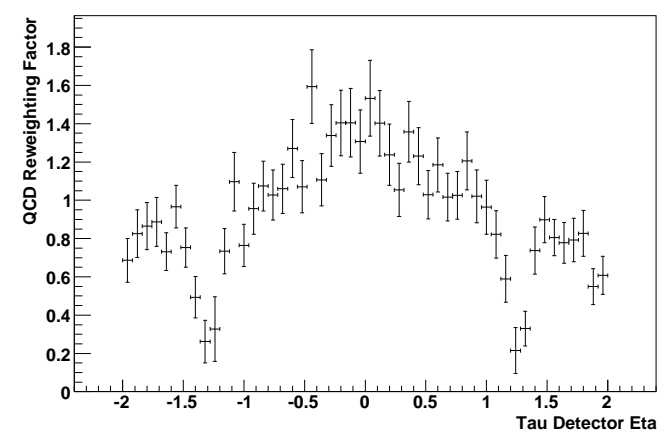

(b) $\eta_{d}(\tau) \mathrm{RW}$

Figure 5.2: Multi-jet background reweighting in (a) $p_{T}(\tau)$ and (b) $\eta_{d}(\tau)$.

\subsubsection{Control Region}

In a similar way to the multi-jet control sample a MC control sample is set up to check the MC is modelling the data well in a region where multi-jet contamination is minimal. To achieve this, the additional requirement $\#_{\mathrm{T}}>80 \mathrm{GeV}$ is made. The tau candidates considered in this sample must match those in the signal sample and so the cut on the hadronic tau neural network is increased to $\tau_{n n_{h}}>0.9$. This yields a sample dominated by $W+$ jets events which determined that no additional normalisation factor is required in the $\mathrm{MC}$ on top of the $k$ factors already applied. This sample is also a test of how well tau identification corrections, taggability and vertex confirmation scale factors are parameterised when compared to data. Figures A.2 and A.3 show kinematic distributions for type 2 and type 1 tau candidate events. Type 2 tau candidate events here benefit from higher statistics and allow a better comparison of data and background shapes.

Figure 5.4 shows the di-jet mass, jet multiplicity, tau transverse momentum and $\mathrm{W}$ transverse mass distributions in the $\mathrm{MC}$ control sample for type 2 tau candidate events. The agreement between predicted background and data is good across most variables. There is a small difference between data and predicted 


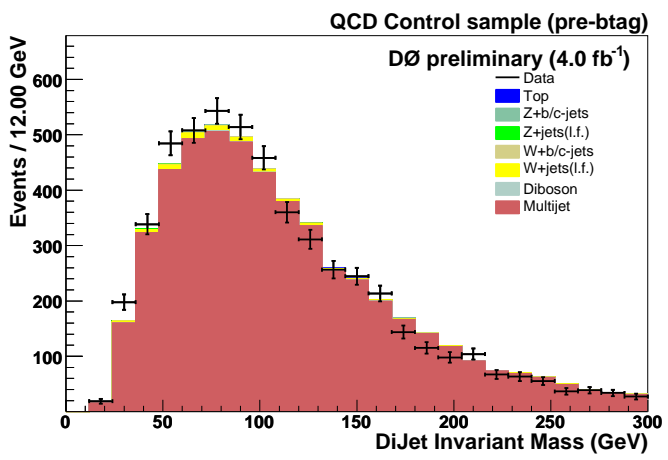

(a) Di-jet Mass

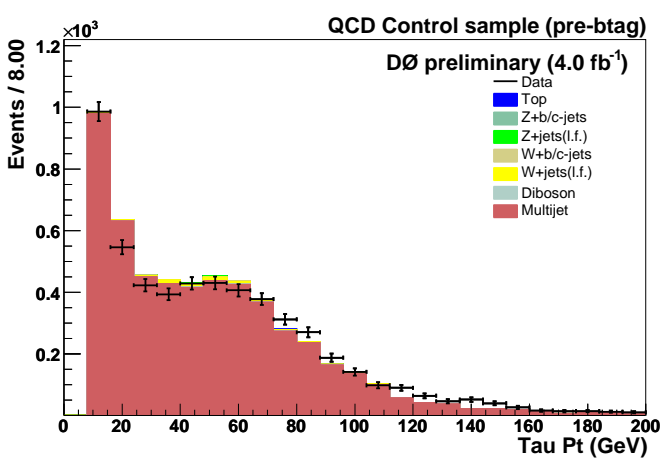

(c) $p_{T}(\tau)$

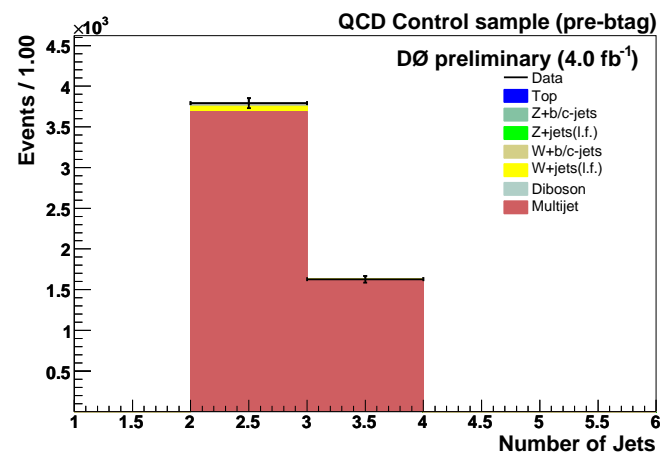

(b) Jet Multiplicity

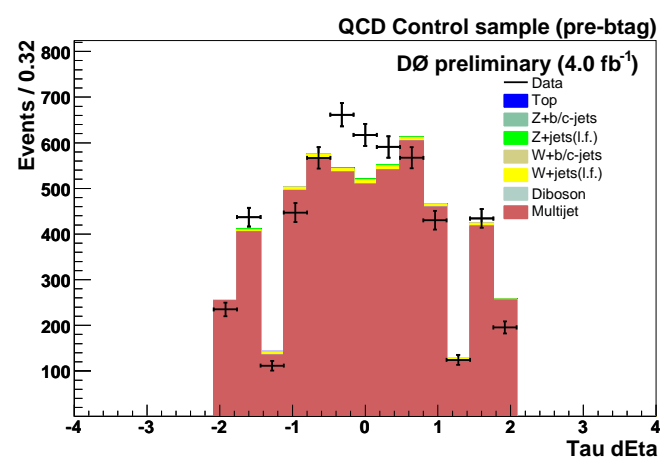

(d) $\eta_{d}(\tau)$

Figure 5.3: (a) Di-jet mass, (b) jet multiplicity, (c) $\tau$ transverse momentum and (d) $\tau$ detector eta in the multi-jet control sample with the reweighted normalisation factor.

background in the jet multiplicity distribution (Figure 5.4(b)). Data show a lower fraction of two-jet over three-jet events than the MC, however, this is not statistically significant. The modelling of the low transverse W mass distribution is less good (Figure 5.4(d)) and this is most obvious in the multi-jet dominated region of the distribution. This is due to the poor modelling of the angle between the tau and the missing transverse energy which is difficult to model at small angles.

Figure 5.5 shows the di-jet mass, jet multiplicity, tau transverse momentum and tau detector eta distributions, for type 1 tau candidate events in the $\mathrm{MC}$ control sample. The type 1 tau candidate events also show a good agreement in the MC control sample within the uncertainties. There is some mis-modelling of 


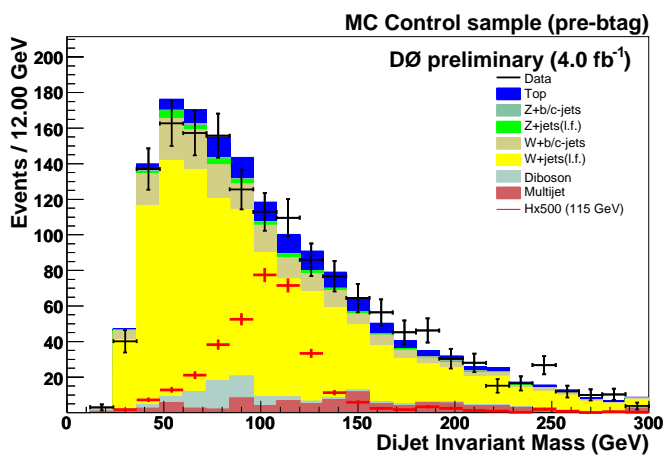

(a) Di-jet Mass

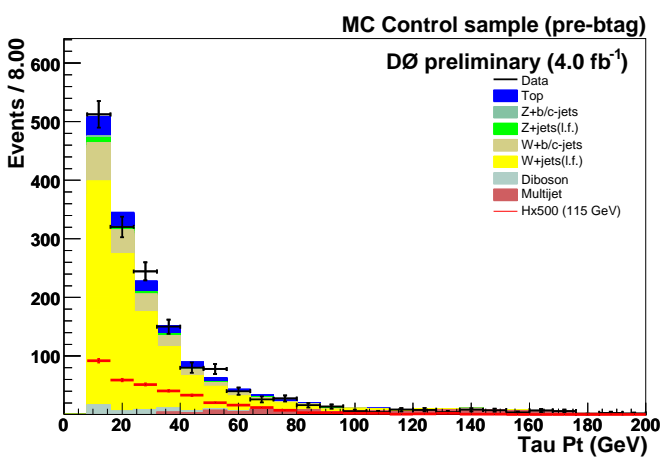

(c) $p_{T}(\tau)$

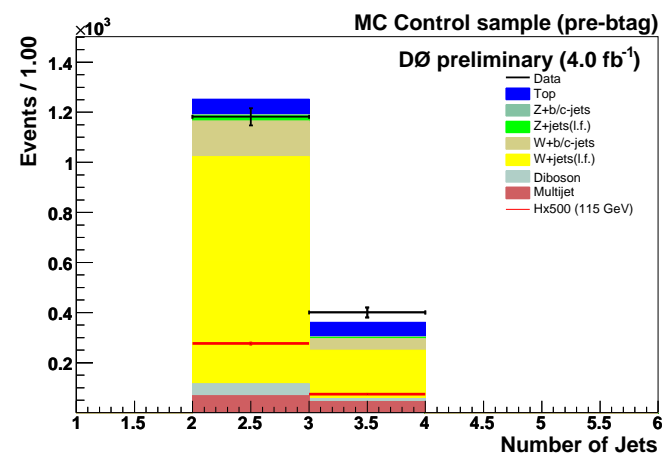

(b) Jet Multiplicity

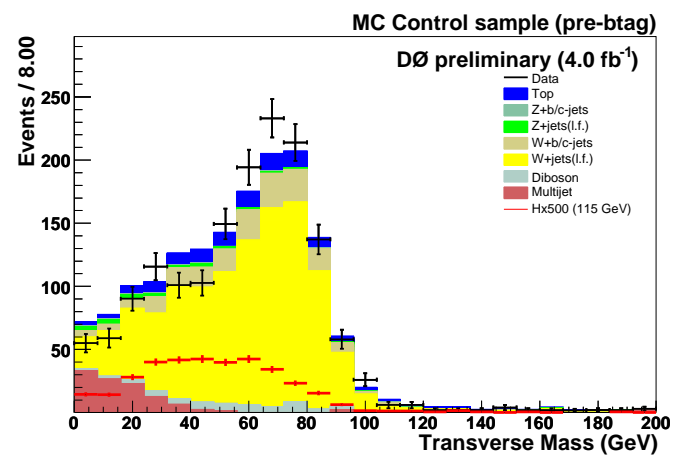

(d) $m_{T}(W)$

Figure 5.4: (a) Di-jet mass, (b) jet multiplicity, (c) $\tau$ transverse momentum and (d) transverse $\mathrm{W}$ mass in the MC control sample for type 2 tau candidate events.

the $\eta_{d}(\tau)$ distribution (Figure 5.5(d)) in this control sample, which is assumed to arise in the central region from low EM fraction hadronic tau candidates, whose energy scale is not well modelled by the simulation. The spikes in the ICD region $\left(1.1<\left|\eta_{d}\right|<1.4\right)$ are dominated by electrons which pass through cracks in the calorimeter and are reconstructed as taus.

\subsection{Modelling in the Analysis Sample}

The normalisation factors derived in the multi-jet control sample (0.28 flat factor + reweighting in $p_{T}(\tau)$ and $\left.\eta_{d}(\tau)\right)$ and the $\mathrm{MC}$ control sample (1.0 as no additional factor is required) are applied to the sample in which the search is 


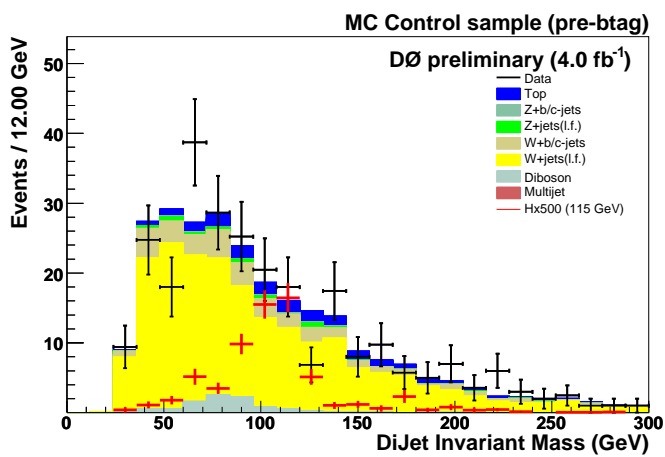

(a) Di-jet Mass

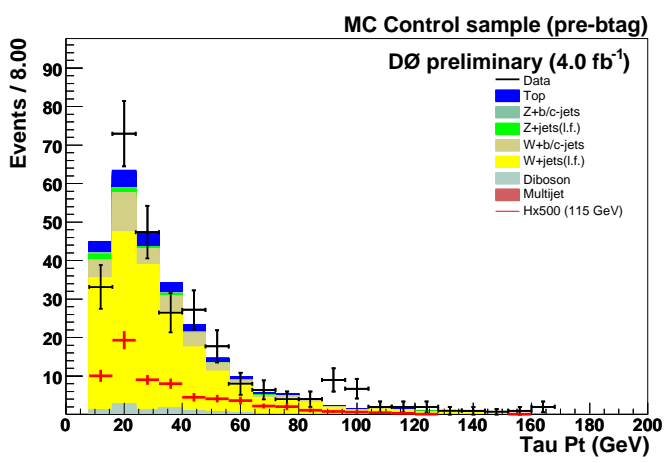

(c) $p_{T}(\tau)$

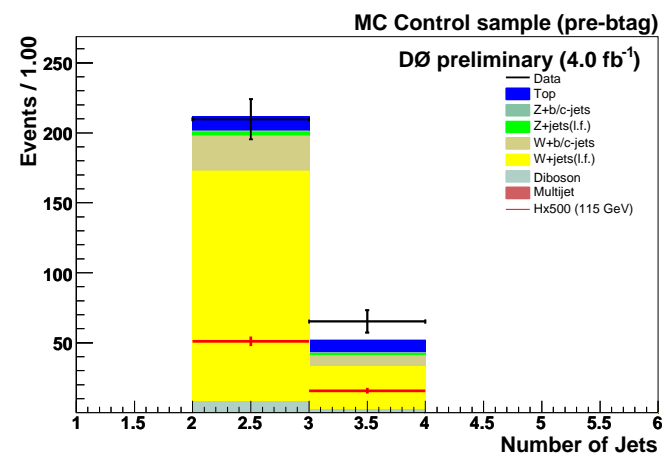

(b) Jet Multiplicity

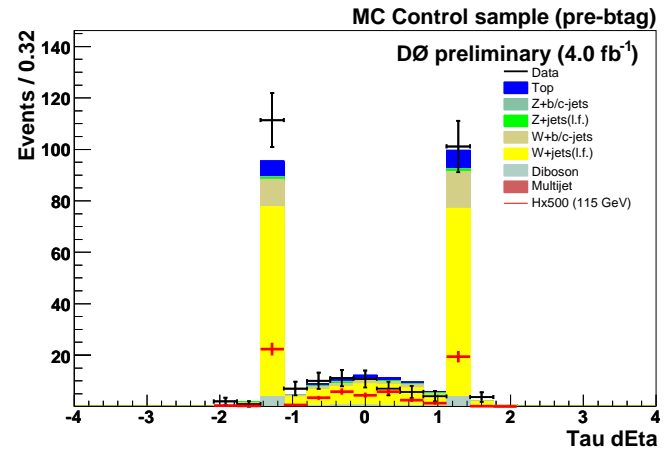

(d) $\eta_{d}(\tau)$

Figure 5.5: (a) Di-jet mass, (b) jet multiplicity, (c) $\tau$ transverse momentum and (d) $\tau$ detector eta in the MC control sample for type 1 tau candidate events.

performed. Initially, the cut on the hadronic tau neural network is raised from the preselection value to $\tau_{n n_{h}}>0.9$ to increase the purity of hadronic tau decays and keep the sample orthogonal to the multi-jet estimate sample. The agreement between data and background is checked at the preselection stage, where the background is dominated by multi-jet events. Once good modelling is achieved, cuts to reduce the number of multi-jet events are applied. When a $W+$ jets dominated background sample is achieved, b-tagging is applied to increase the heavy flavour content of the sample and improve the signal to background ratio. 


\subsubsection{Preselection}

At the preselection stage the data sample is dominated by jets faking hadronic tau decays. This sample is therefore used to test how well the multi-jet estimate and reweighting in the multi-jet control region performs in the signal sample topology. Figure 5.6 shows the di-jet mass, tau transverse momentum,

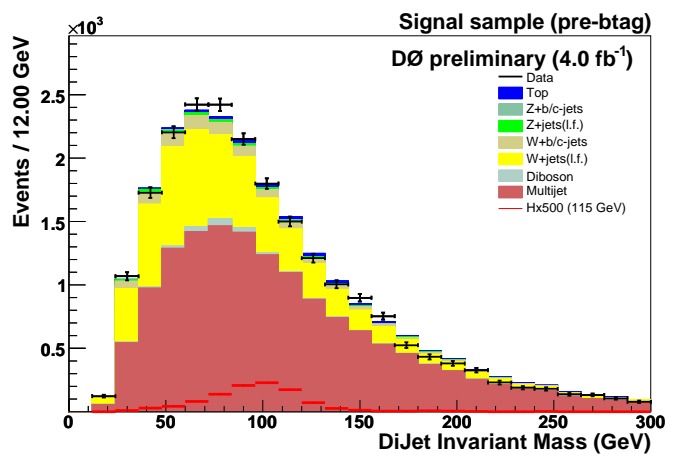

(a) Di-jet mass

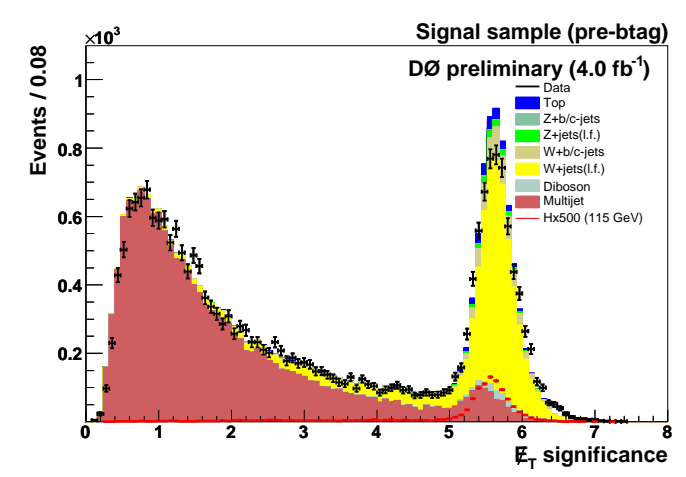

(c) $\not_{\mathrm{T}_{\mathrm{SIG}}}$

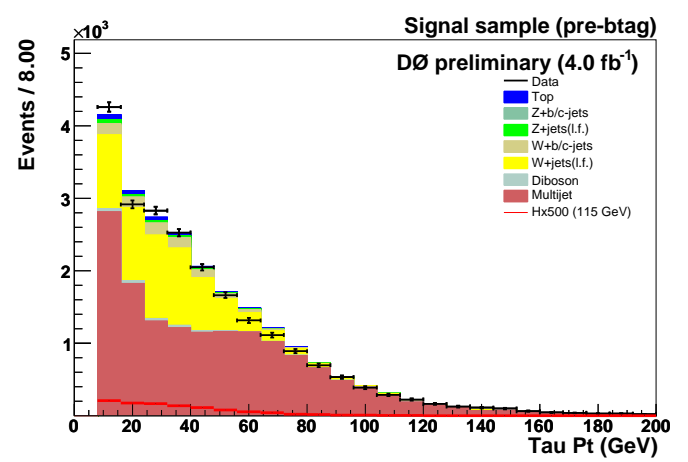

(b) $p_{T}(\tau)$

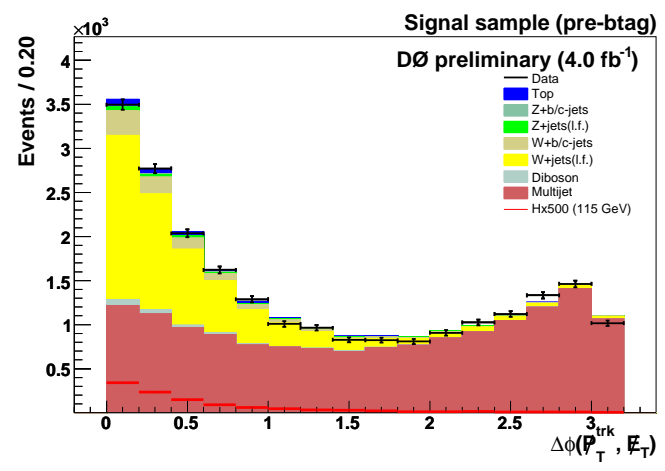

(d) $\Delta \phi\left(\not P_{\mathrm{T}}, \not_{\mathrm{T}}\right)$

Figure 5.6: (a) Di-jet mass, (b) $\tau$ transverse momentum, (c) missing transverse energy significance and (d) $\Delta \phi\left(\not P_{\mathrm{T}}, \not_{\mathrm{T}}\right)$ in the analysis sample at the preselection stage for type 2 tau candidate events.

missing transverse energy significance and $\Delta \phi\left(\not \not_{\mathrm{T}}, \not_{\mathrm{T}}\right)$ for type 2 tau candidate events. The missing transverse energy significance distribution (Figure 5.6(c)) shows good separation between signal and the multi-jet background. Good separation between signal and multi-jet background is also found in the $\Delta \phi\left(\not P_{\mathrm{T}}, \not_{\mathrm{T}}\right)$ variable (Figure 5.6(d)), where $\not_{\mathrm{T}}$ is the missing energy from tracks only. Addi- 
tional kinematic distributions for type 2 tau candidate events at the preselection stage are shown in Figure A.4.

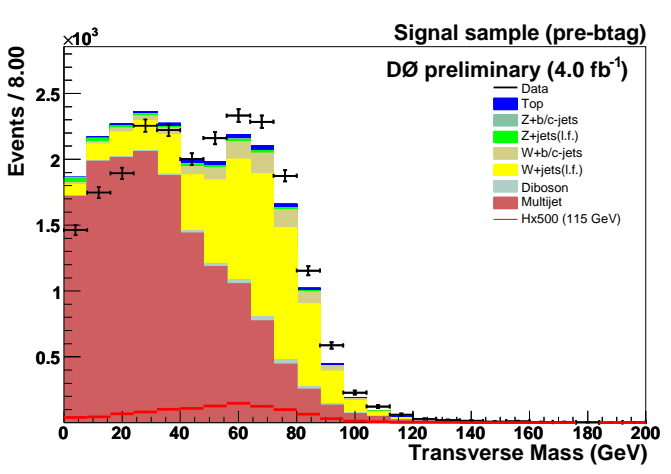

(a) $m_{T}(W)$

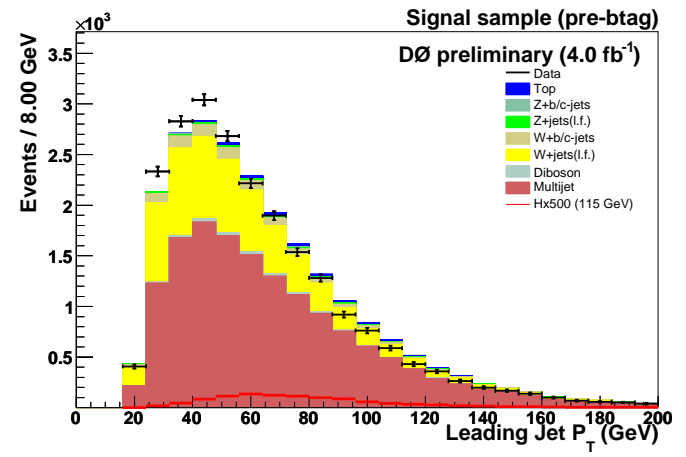

(b) $p_{T}($ jet 1$)$

Figure 5.7: (a) Transverse $\mathrm{W}$ mass and (b) leading jet transverse momentum distributions in the analysis sample at the preselection stage for type 2 tau candidate events.

There are some variables which are not well modelled at this stage of the analysis, for example the transverse W mass distribution (Figure 5.7(a)), especially at low values of $m_{T}(W)$. This issue arises from a mis-modelling of the angle $\Delta \phi\left(\tau, E_{\mathrm{T}}\right)$ at small angles where the $\tau$ and $\mathscr{E}_{\mathrm{T}}$ are aligned. This is a problem in the multi-jet estimate as the isolation requirement for the tau candidates in the multi-jet estimate sample is looser than that in the analysis sample. This causes the angle to be less well defined as it approaches zero. There is a small shape difference between the data and predicted background in the leading jet $p_{T}$ distribution (Figure 5.7(b)) caused by the multi-jet estimate having a broader $p_{T}$ spectrum than events in the analysis sample.

For type 1 tau candidate events the di-jet mass (Figure 5.8(a)) and tau transverse momentum (Figure 5.8(b)) are both well modelled by the background and show that the statistics in the type 1 tau sample are less than in the type 2 tau sample. As was found in the type 2 tau distributions, discrimination between the signal and the multi-jet background is very good in the $\mathbb{E}_{\mathrm{T}_{\mathrm{SIG}}}($ Figure $5.8(\mathrm{c})$ ) and $\Delta \phi\left(\not P_{\mathrm{T}}, \not_{\mathrm{T}}\right)($ Figure 5.8(d)) distributions. 


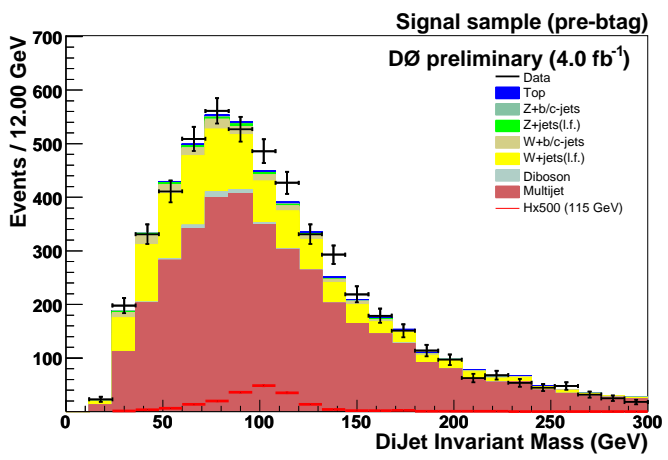

(a) Di-jet Mass

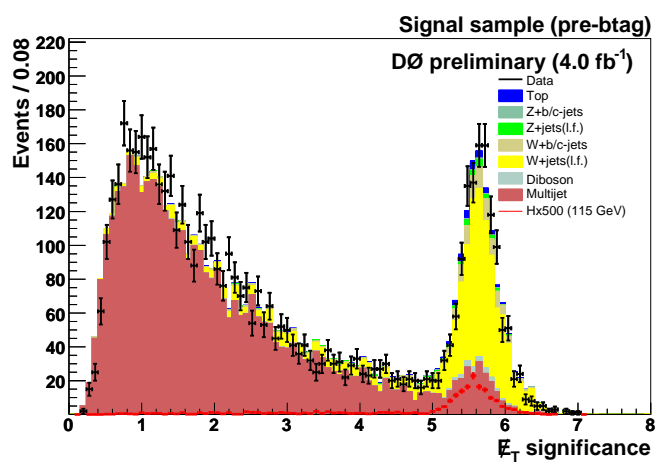

(c) $\not_{T_{\text {SIG }}}$

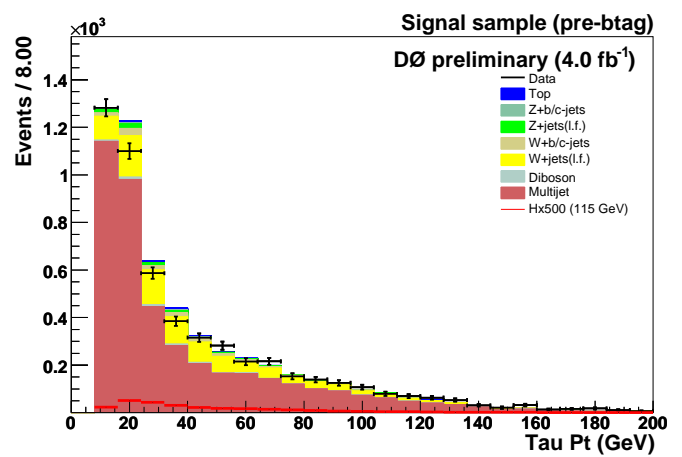

(b) $p_{T}(\tau)$

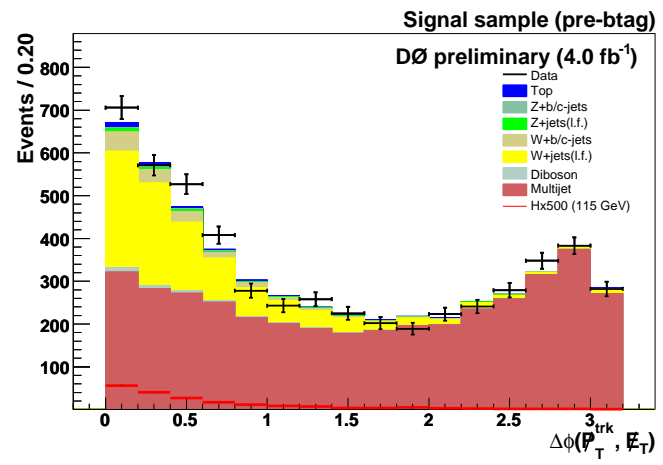

(d) $\Delta \phi\left(\not P_{\mathrm{T}}, \notin_{\mathrm{T}}\right)$

Figure 5.8: (a) Di-jet mass, (b) $\tau$ transverse momentum, (c) missing transverse energy significance and (d) $\Delta \phi\left(\not P_{\mathrm{T}}, \not_{\mathrm{T}}\right)$ in the analysis sample at the preselection stage for type 1 tau candidate events.

The central region $\left(\left|\eta_{d}\right|<1.1\right)$ of the tau detector eta distribution (Figure $5.9(\mathrm{a}))$ is not perfectly modelled at this stage. The slight central excess is consistent with that observed in the multi-jet control sample after reweightings are applied. The transverse $\mathrm{W}$ mass distribution (Figure 5.9(b)) shows similar shape discrepancies as the type 2 taus at this stage. It is again consistent with a multi-jet modelling issue, as the tail of the distribution (dominated by MC) is well modelled. Additional kinematic distributions for type 1 tau candidate events at the preselection stage are shown in Figure A.5. 


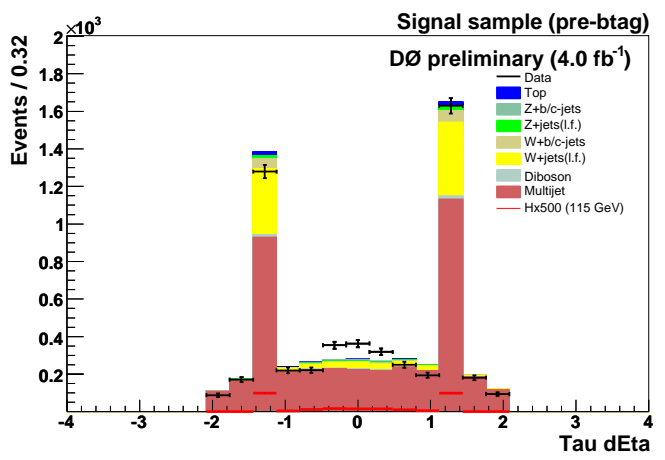

(a) $\eta(\tau)$

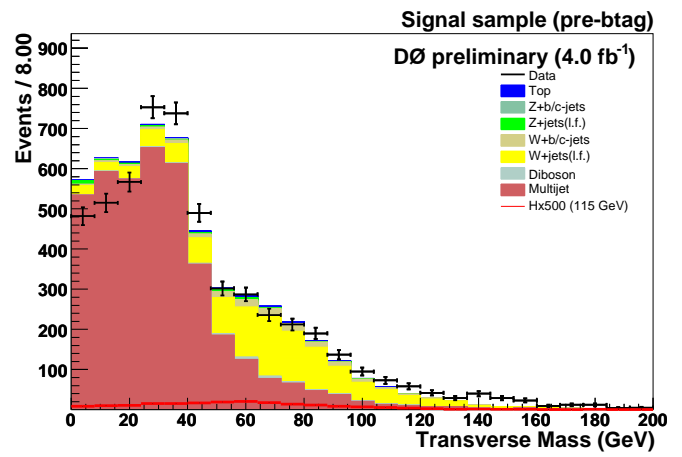

(b) $m_{T}(W)$

Figure 5.9: (a) $\tau$ detector eta and (b) transverse $W$ mass in the analysis sample at the preselection stage for type 1 tau candidate events.

\subsubsection{Selection}

To improve the signal to background ratio in the sample and remove much of the dominant multi-jet background at this stage, further cuts are implemented as follows:

- $\Delta \phi\left(\not P_{\mathrm{T}}, \not_{\mathrm{T}}\right)<2.0$

- $E_{\mathrm{T}_{\mathrm{SIG}}}>4.5$

These cuts are motivated by the preselection stage distributions (Figures $5.6(\mathrm{c}, \mathrm{d})$ and $5.8(\mathrm{c}, \mathrm{d}))$ and are around $90 \%$ efficient on the signal sample, yet remove over $80 \%$ of the multi-jet background. After these cuts the resulting samples are dominated by $W+$ jets events (where the jets are dominated by light flavour $u, d, s$ quarks).

The agreement in type 2 tau candidate events after the selection cuts is good across many variables (full set of variables shown in Figure A.6). The modelling of variables, such as the transverse momentum of the tau (Figure 5.10(a)), is particularly informative, as the double peak structure shows the hadronic tau decays at lower values of transverse momentum decrease and then the higher 


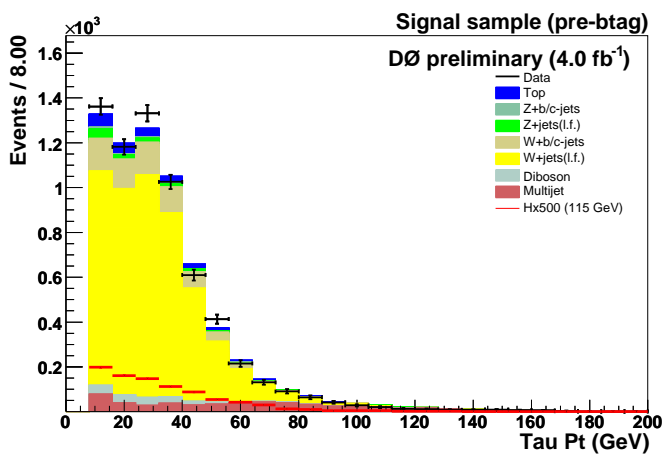

(a) $p_{T}(\tau)$

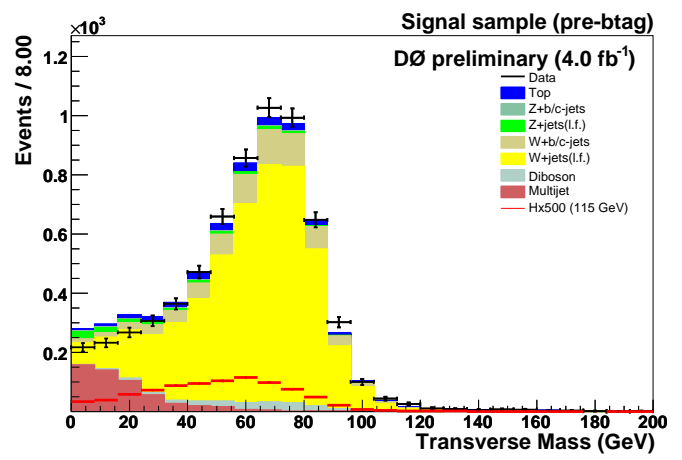

(b) $m_{T}(W)$

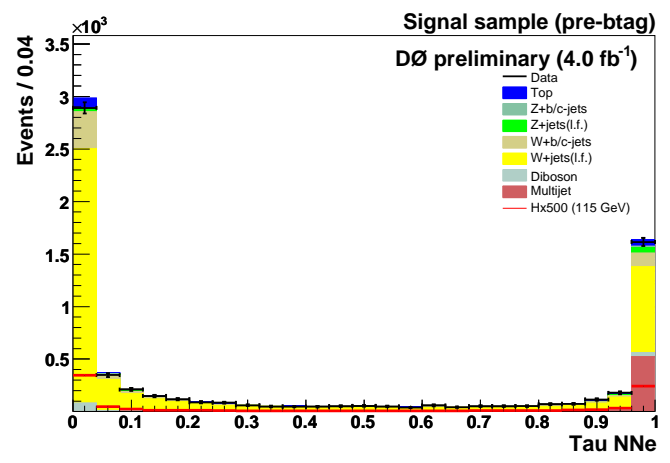

(c) $\tau_{n n_{\text {elec }}}$

Figure 5.10: (a) $\tau$ transverse momentum, (b) transverse $\mathrm{W}$ mass and (c) $\tau_{n n_{\text {elec }}}$ in the analysis sample at the selection stage for type 2 tau candidate events.

transverse momentum electrons peak around $30 \mathrm{GeV}$. Another distribution that is sensitive to the difference between electron fakes and hadronic tau decays in the background is the transverse $\mathrm{W}$ mass distribution (Figure 5.10(b)). The electrons faking hadronic tau decays in the background peak around $80 \mathrm{GeV}$ (the $W$ mass), whereas the hadronic tau decays peak at lower values (around $50 \mathrm{GeV}$ ) and are smeared across a wider range. This occurs due to the additional neutrinos lost in the hadronic tau decay, so not all of the $\mathrm{W}$ decay products enter into the reconstruction of the transverse mass. Although the type 2 tau sample consists of both electrons and hadronic tau decays, it is possible to separate them for the purpose of understanding the individual backgrounds via a dedicated neural network $\left(\tau_{n n_{\text {elec }}}\right)$. The $\tau_{n n_{\text {elec }}}$ distribution peaks at zero for electron-like tau 
candidates and at one for hadronic tau-like candidates. The $\tau_{n n_{\text {elec }}}$ distribution for type 2 tau candidates at the selection stage is shown in Figure 5.10(c) and shows that the sample consists of around two thirds electrons and one third hadronic tau decays. The multi-jet background is peaked towards one, as hadronic tau decays are faked by jets more readily than electrons are faked by jets.

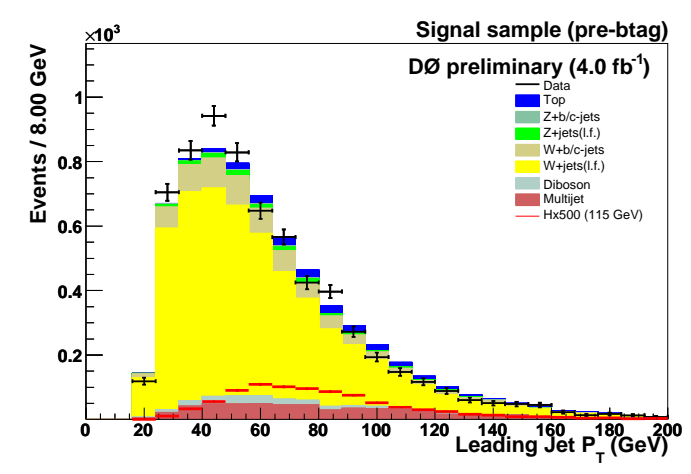

(a) $p_{T}(j e t 1)$

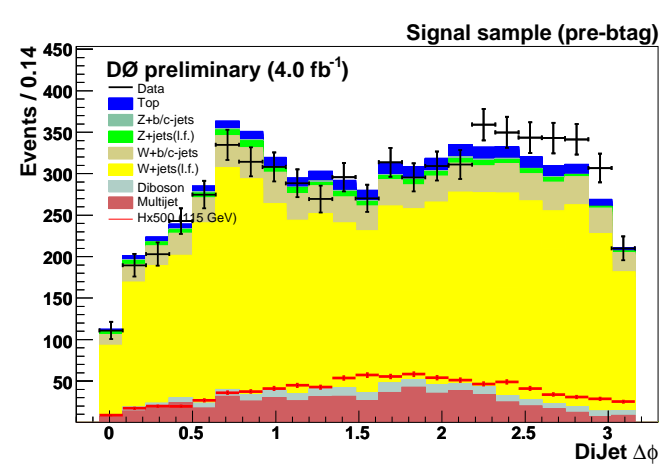

(b) $\Delta \phi($ jet 1, jet 2$)$

Figure 5.11: (a) Leading jet transverse momentum and (b) $\Delta \phi($ jet 1 , jet2) in the analysis sample at the selection stage for type 2 tau candidate events.

The slight excess of data events at low values of the leading jet $p_{T}$ can still be observed after the selection cuts (Figure 5.11(a)). This feature of the leading jet $p_{T}$ distribution also affects the angle between the two leading jets in the event (Figure 5.11(b)) and manifests itself as an excess of back-to-back jet events.

The agreement between data and predicted background in type 1 tau candidate events after the selection is generally good (full set of variables shown in Figure A.7). The tau detector $\eta$ distribution (Figure 5.12(a)) is improved as most of the multi-jet background has been removed by the selection. The transverse $\mathrm{W}$ mass distribution (Figure 5.12(b)) shows some discrimination between the electroweak events and the remaining multi-jet events. The electrons faking hadronic tau decays in the ICD region of the detector (Figure 5.12(a)) reconstruct the real $W$ mass, but the hadronic tau decays peak at lower values and are faked by jets far more frequently than the electrons, which explains why the remaining 


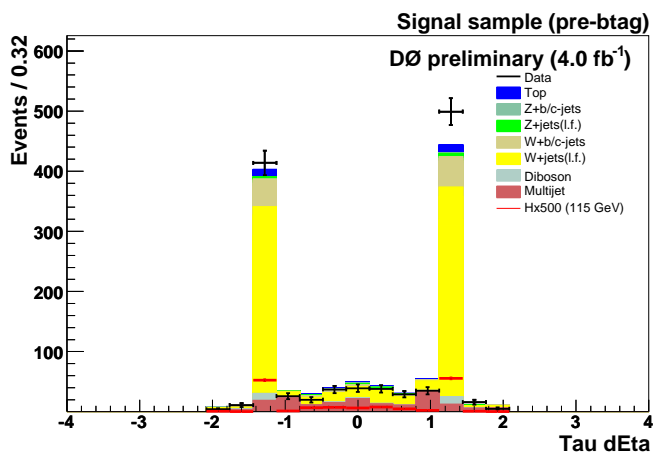

(a) $\eta(\tau)$

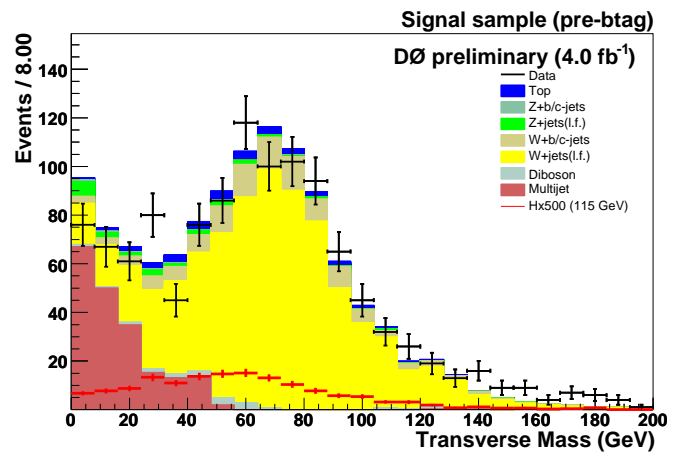

(b) $m_{T}(W)$

Figure 5.12: (a) $\tau$ detector eta and (b) transverse $W$ mass in the analysis sample at the selection stage for type 2 tau candidate events.

multi-jet events are found at low values of the transverse mass.

The fraction of multi-jet events in the background is higher for type 1 taus than their type 2 counterparts. This is due to the additional EM sub-cluster requirement in type 2 tau candidates, which increases the EM fraction of the tau candidates and removes many of the jet fakes. Typically type 1 taus have an EM fraction below 0.3 , which leads to a higher number of jet fakes.

\subsubsection{One b-tag selection}

Although the best sensitivity to the Higgs signal is expected in a two b-tag configuration, it is still possible to gain sensitivity by studying a sample where one of the jets fails the $L 3$ b-tagging criteria. This sample is formed where one of the two leading transverse momentum jets in the event passes the VeryTight operating point and the other fails the L3 operating point conditions. It is therefore orthogonal to the sample where both of the leading jets are required to be tagged with at least the $L 3$ operating point.

Another benefit of the one b-tag sample is that there are greater statistics than in the two b-tag sample which allows for better constraints on the systematic 
uncertainties when the fitting is performed later in the analysis (See Section 6.3). It also offers greater statistics to check whether the modelling of a $W / Z+h e a v y$ flavour sample is as good as the $W / Z+$ light flavour samples at the selection stage.

As the limits are to be set in exclusive jet multiplicity bins, the distributions after b-tagging are shown here exclusively for two and three jet events.

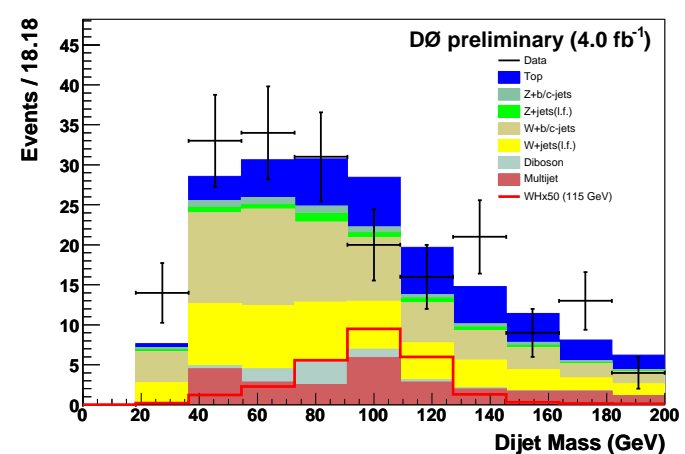

(a) Di-jet mass, 2 jets

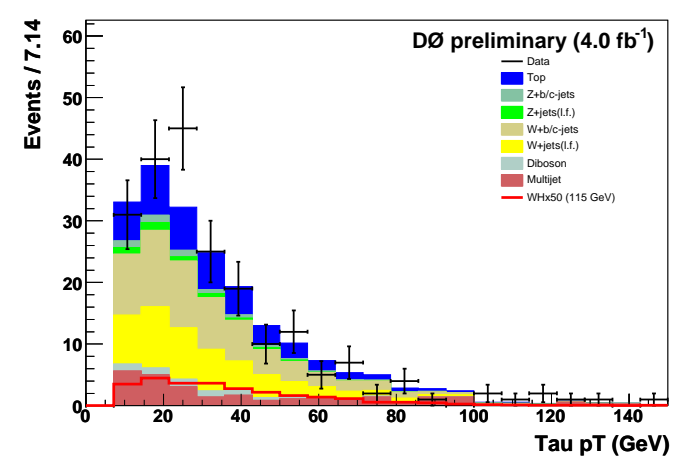

(c) $p_{T}(\tau), 2$ jets

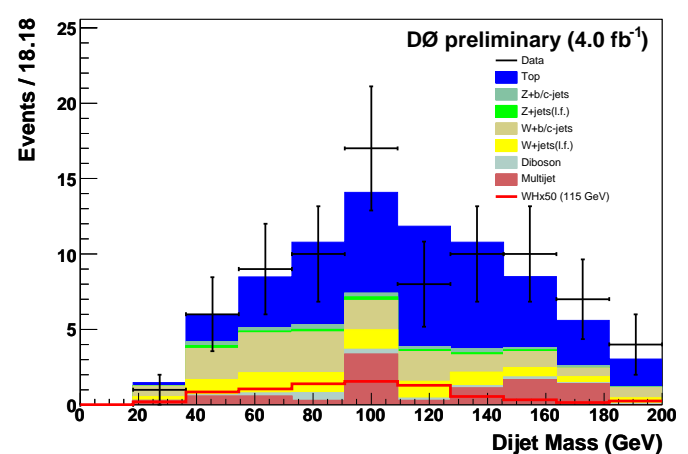

(b) Di-jet mass, 3 jets

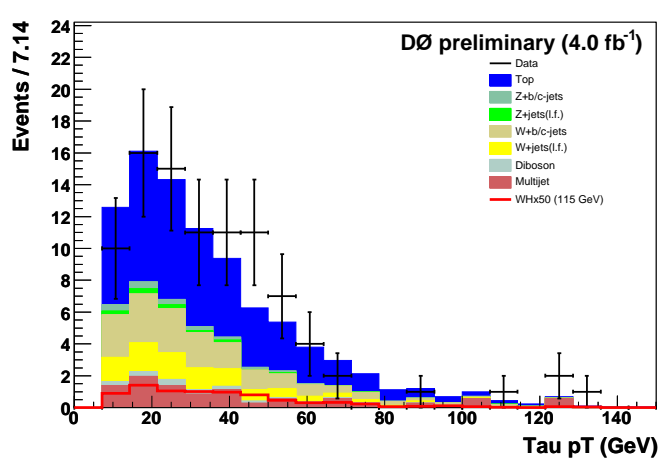

(d) $p_{T}(\tau), 3$ jets

Figure 5.13: Di-jet mass and $\tau$ transverse momentum in the analysis sample at the one b-tag selection stage for type 2 tau candidate events with (a,c) 2 associated jets and (b,d) 3 associated jets.

The background composition changes dramatically when a b-jet is required in the final state. Figure 5.13 shows the di-jet mass for type 2 tau candidate events and the tau transverse momentum for events with two associated jets and three associated jets. The tau transverse momentum distribution is shown to verify that the shape agrees after the b-tag selection in tau based variables, and not just jet 
variables. Although the two jet samples are still dominated by $W+$ jets, the heavy flavour content of the associated jets is significantly increased. Additionally, $t \bar{t}$ events now make up a significant portion of the background. When three jets are required in the sample, the background is dominated by $t \bar{t}$ events, as these events have a high jet multiplicity. The signal is now scaled by a factor of fifty (rather than five hundred), which indicates how much tagging a b-jet has increased the sensitivity to a Higgs boson signal.

The agreement between data and predicted background in type 2 tau candidate events with two associated jets, is generally very good in both the integrated number of events and the shape of variables (Figure A.8). The agreement in type 2 tau events with three associated jets is similarly good and the event yields are summarised in Table 5.2.

Figure 5.14 shows the di-jet mass for type 1 tau candidate events and the tau transverse momentum for events with two associated jets and three associated jets. As the statistics in type 1 tau candidate events are much lower than for type 2 tau events it is difficult to draw conclusions about shape agreement, but there is no obvious sign of disagreement in these distributions, except a slight data deficit in the three jet events. The full range of kinematic variables after requiring one b-tagged jet are shown in Figures A.8 and A.9 for type 2 candidate events and in Figures A.10 and A.11 for type 1 candidate events.

\section{One b-tag Event Yields}

Table 5.2 shows the data and background yields after the one b-tag selection for type 1 and type 2 tau candidate events with two and three associated jets. The agreement between the predicted background and the observed data is good across all channels. Only statistical uncertainties are considered here. The dominant background contribution comes from $W+$ jets, with a large fraction being heavy 


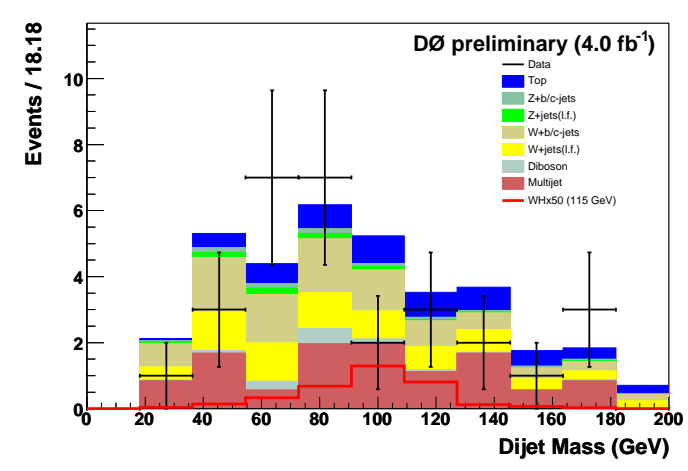

(a) Di-jet mass, 2 jets

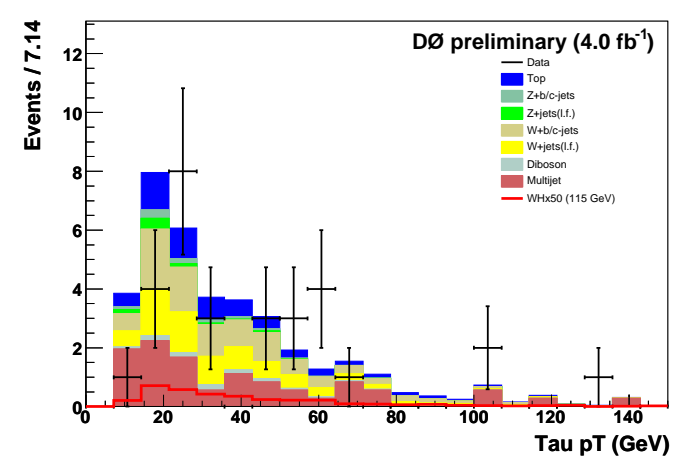

(c) $p_{T}(\tau), 2$ jets

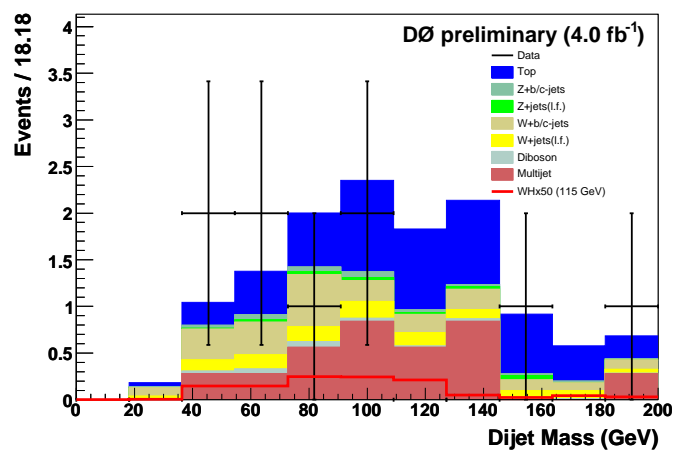

(b) Di-jet mass, 3 jets

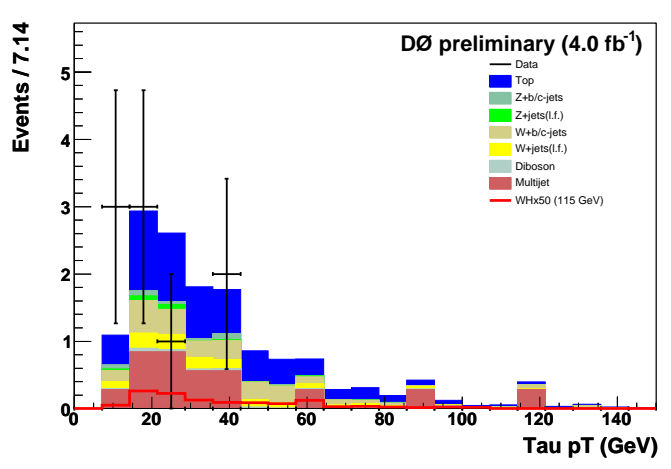

(d) $p_{T}(\tau), 3$ jets

Figure 5.14: Di-jet mass and $\tau$ transverse momentum in the analysis sample at the one b-tag selection stage for type 1 tau candidate events with (a,c) 2 associated jets and (b,d) 3 associated jets.

flavour jets which is the irreducible background to this search. A significant number of $t \bar{t}$ events comprise the background when b-tagging selections are made, this background increases as the jet multiplicity in the event increases.

\subsubsection{Two b-tag selection}

The two b-tag sample is most sensitive to the signal as the Higgs decays to $b \bar{b}$. Instead of requiring two high quality b-jets with a tight operating point, two fairly loose b-tagged jets are required. It is beneficial to retain as much signal as possible for the training of the boosted decision tree, where background can be further rejected. By requiring two b-jets in the event the sample is further 


\begin{tabular}{|c|c|c|c|c|}
\hline Sample & Type 1, 2 jet & Type 2, 2 jet & Type 1, 3 jet & Type 2, 3 jet \\
\hline Signal & $0.072 \pm 0.001$ & $0.543 \pm 0.001$ & $0.024 \pm 0.001$ & $0.164 \pm 0.001$ \\
Diboson & $1.12 \pm 0.03$ & $6.77 \pm 0.08$ & $0.30 \pm 0.01$ & $1.88 \pm 0.02$ \\
Multi-jet & $12.3 \pm 1.5$ & $26.9 \pm 2.8$ & $4.02 \pm 0.95$ & $10.41 \pm 1.43$ \\
Top & $5.73 \pm 0.05$ & $42.55 \pm 0.15$ & $5.75 \pm 0.05$ & $46.32 \pm 0.17$ \\
Wbb/cc & $9.07 \pm 0.20$ & $63.31 \pm 0.57$ & $2.20 \pm 0.02$ & $16.85 \pm 0.15$ \\
Wjj & $7.74 \pm 0.19$ & $50.23 \pm 0.50$ & $1.42 \pm 0.03$ & $10.31 \pm 0.10$ \\
Zbb & $0.85 \pm 0.06$ & $5.56 \pm 0.14$ & $0.35 \pm 0.02$ & $2.09 \pm 0.05$ \\
Zjj & $0.83 \pm 0.10$ & $4.84 \pm 0.18$ & $0.21 \pm 0.03$ & $1.27 \pm 0.05$ \\
\hline Sum Bkgd & $37.64 \pm 1.55$ & $200.2 \pm 2.94$ & $14.25 \pm 0.95$ & $89.13 \pm 1.45$ \\
Data & 31 & 210 & 9 & 93 \\
\hline s/ $\sqrt{b}$ & 0.012 & 0.038 & 0.0064 & 0.0174 \\
\hline
\end{tabular}

Table 5.2: One b-tagged samples event yield table. The uncertainties given are statistical only and are rounded up for the errors on the signal sample.

purged of multi-jet events, and the heavy flavour contribution is increased over the single b-tag sample (shown by Tables 5.2 and 5.3).

Figure 5.15 shows the di-jet mass distribution for events with two then three associated jets. It also shows the tau transverse momentum for both samples. Both of these important kinematic variables are well modelled by the background for events with two associated jets. When three associated jets are required in the type 2 tau sample, $t \bar{t}$ events dominate the background. There appears to be a systematic deficit in the data across a few consecutive bins in the di-jet mass distribution (Figure 5.15(b)). This deficit does not appear in any other obvious region in any other variable (see Figure A.13), so it is assumed to be a statistical effect. The di-jet mass distribution yields good discrimination between the signal and electroweak backgrounds. This was used in the previous version of this analysis as the final discriminant [50].

Other variables such as $H_{T}$ (Figure 5.16(a)) also exhibit some discriminating power between signal and background. This is most important for two jet events where the $t \bar{t}$ contribution is small (these $t \bar{t}$ events normally have high $H_{T}$ due to higher transverse momentum jets in the final state). This adds to the motivation 


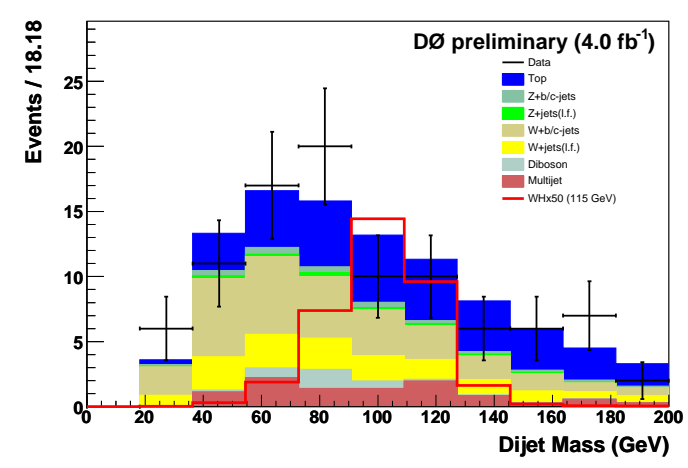

(a) Di-jet mass, 2 jets

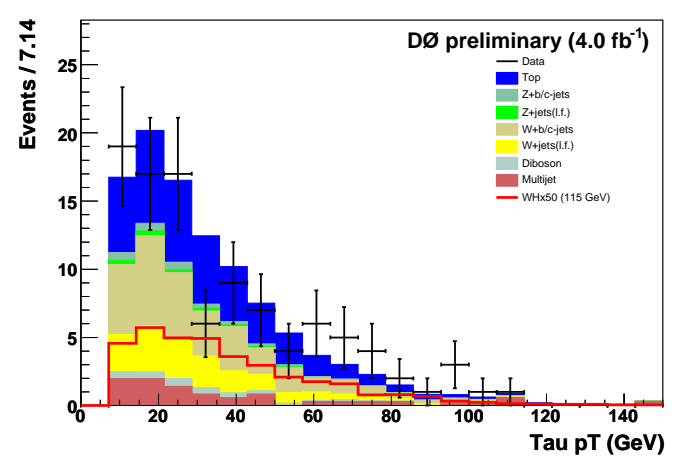

(c) $p_{T}(\tau), 2$ jets

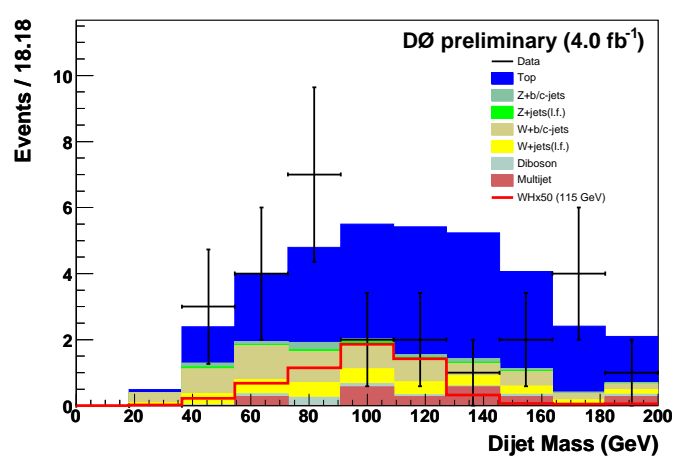

(b) Di-jet mass, 3 jets

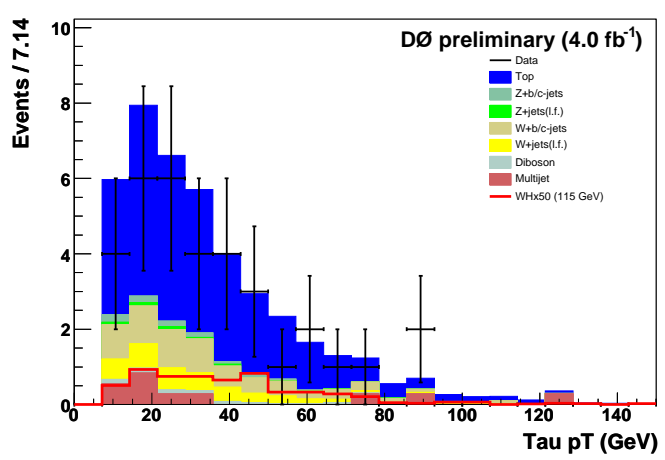

(d) $p_{T}(\tau), 3$ jets

Figure 5.15: Di-jet mass and $\tau$ transverse momentum in the analysis sample at the two b-tag selection stage for type 2 tau candidate events with (a,c) 2 associated jets and (b,d) 3 associated jets.

for the use of a multivariate over a single variable technique for final discrimination in the analysis.

Figure 5.17 shows the tau transverse momentum distribution for type 1 tau candidate events with two then three associated jets. The agreement in these samples is generally good in the integrated number of events, but the statistics are becoming too small to conclude much about the shape agreement within uncertainties. There is still a significant fraction of multi-jet events in the background (Table 5.3), but less than in the single b-tag sample (Table 5.2).

This can be observed in the $\eta_{d}(\tau)$ distribution (Figure 5.18) where the fraction of multi-jet events in the ICD region is reduced. When three associated jets are 


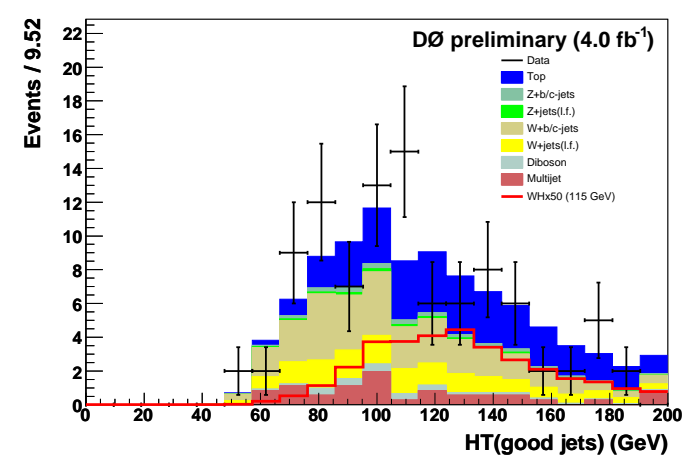

(a) $H_{T}, 2$ jets

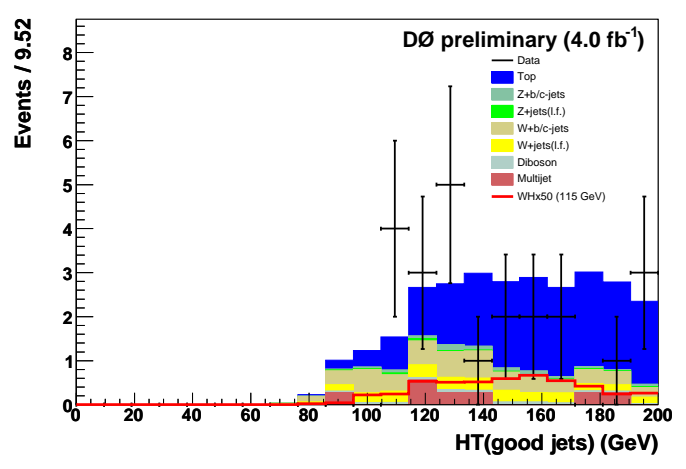

(b) $H_{T}, 3$ jets

Figure 5.16: Vector sum of jet energies in the analysis sample at the two b-tag selection stage for type 2 tau candidate events with (a) 2 associated jets and (b) 3 associated jets.

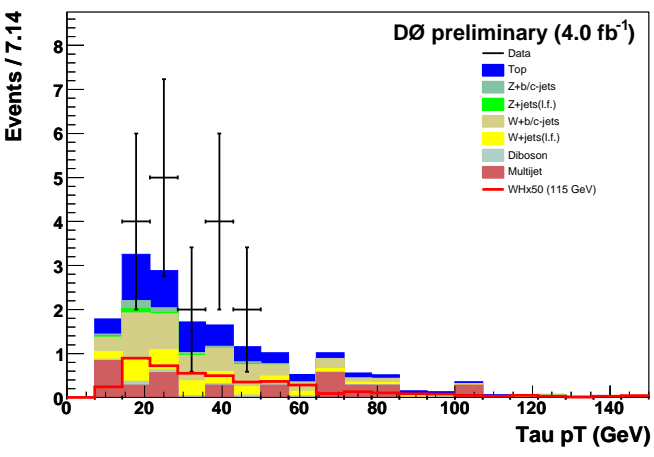

(a) $p_{T}(\tau), 2$ jets

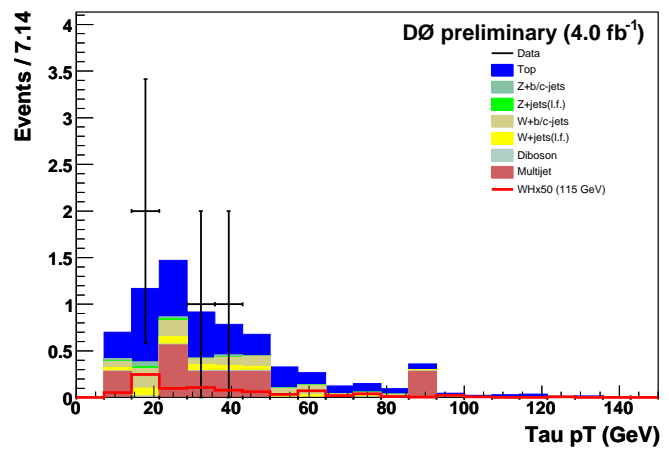

(b) $p_{T}(\tau), 3$ jets

Figure 5.17: Tau transverse momentum in the analysis sample at the two b-tag selection stage for type 1 tau candidate events with (a) 2 associated jets (b) 3 associated jets.

required in the type 1 tau sample, similar features are observed as for type 2 tau events. However, some problems with the multi-jet estimate are encountered, as the statistics in the sample becomes low, and a "spiky" structure to the multi-jet background is observed. The multi-jet shape is not considered for the training of the analysis multivariate technique, to avoid training on these statistical fluctuations. Additional kinematic distributions after requiring two b-tagged jets are shown in Figures A.12 and A.13 for type 2 candidate events and in Figures A.14 and A.15 for type 1 candidate events. 


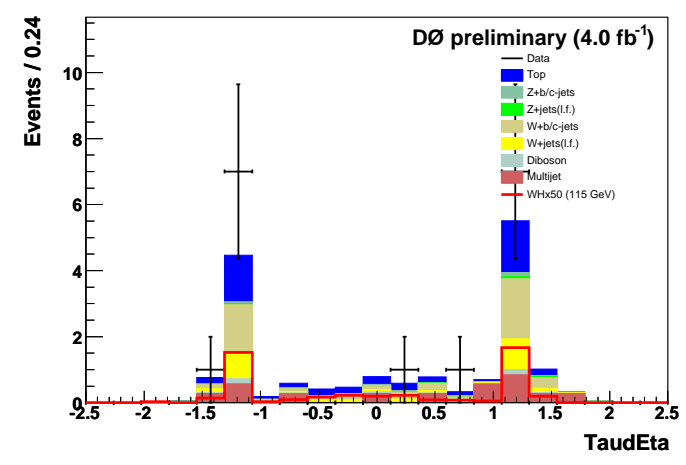

(a) $\eta_{d}(\tau), 2$ jets

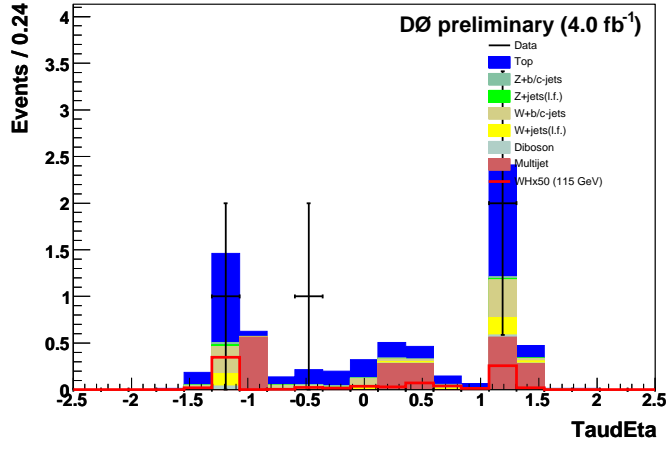

(b) $\eta_{d}(\tau), 3$ jets

Figure 5.18: $\tau$ detector eta in the analysis sample at the two b-tag selection stage for type 1 tau candidate events with (a) 2 associated jets and (b) 3 associated jets.

\section{Two b-tag Event Yields}

Table 5.3 shows the data and background yields for the two b-tag selection for type 1 and type 2 tau candidate events with two and three associated jets. The agreement between the data and predicted background is again reasonably good and the background composition is very similar to the one b-tag selection. The fraction of $t \bar{t}$ events in the background has increased relative to $W+$ heavy flavour jets, as the likelihood of a $W+c \bar{c}$ event containing two b-tagged jets is lower than the probability of only one charm jet being b-tagged $(t \bar{t}$ events contain two b-quarks in the final state). 


\begin{tabular}{|c|c|c|c|c|}
\hline Sample & Type 1, 2 jet & Type 2,2 jet & Type 1, 3 jet & Type 2, 3 jet \\
\hline Signal & $0.091 \pm 0.001$ & $0.716 \pm 0.001$ & $0.017 \pm 0.001$ & $0.116 \pm 0.001$ \\
Diboson & $0.47 \pm 0.01$ & $3.23 \pm 0.04$ & $0.086 \pm 0.003$ & $0.73 \pm 0.04$ \\
Multi-jet & $3.74 \pm 1.02$ & $10.86 \pm 1.74$ & $2.03 \pm 0.75$ & $3.41 \pm 0.97$ \\
Top & $4.75 \pm 0.04$ & $38.01 \pm 0.11$ & $3.51 \pm 0.03$ & $27.46 \pm 0.08$ \\
Wbb/cc & $4.76 \pm 0.17$ & $30.84 \pm 0.43$ & $0.94 \pm 0.03$ & $6.17 \pm 0.08$ \\
Wjj & $2.58 \pm 0.04$ & $16.82 \pm 0.16$ & $0.52 \pm 0.01$ & $3.64 \pm 0.04$ \\
Zbb & $0.50 \pm 0.05$ & $2.87 \pm 0.09$ & $0.133 \pm 0.001$ & $0.93 \pm 0.03$ \\
Zjj & $0.24 \pm 0.02$ & $1.46 \pm 0.05$ & $0.065 \pm 0.007$ & $0.37 \pm 0.01$ \\
\hline Sum Bkgd & $17.04 \pm 1.04$ & $104.1 \pm 1.81$ & $7.28 \pm 0.75$ & $42.7 \pm 0.98$ \\
Data & 17 & 103 & 4 & 35 \\
\hline s/ $\sqrt{b}$ & 0.22 & 0.07 & 0.0063 & 0.0178 \\
\hline
\end{tabular}

Table 5.3: Two b-tagged samples event yield table. The uncertainties given are statistical only and are rounded up for the errors on the signal sample. 


\section{Chapter 6}

\section{Search for Higgs boson in the $\tau \nu b \bar{b}$ Channel}

\subsection{Multivariate Analysis}

To increase the signal to background ratio in the analysis sample, a boosted decision tree (BDT) is trained using the samples after both one and two b-tag selections.

\subsubsection{Decision Trees}

A decision tree is a type of machine learning technique which constructs a series of cuts to maximise the signal to background ratio in a sample. An example is shown in Figure 6.1 [51]. In this figure the input variable cuts known as nodes are shown in blue and the terminal input nodes or leaves are shown in green. After each cut the purity $P$ of the sample is considered,

$$
P=\frac{N_{s}}{N_{s}+N_{b}}
$$




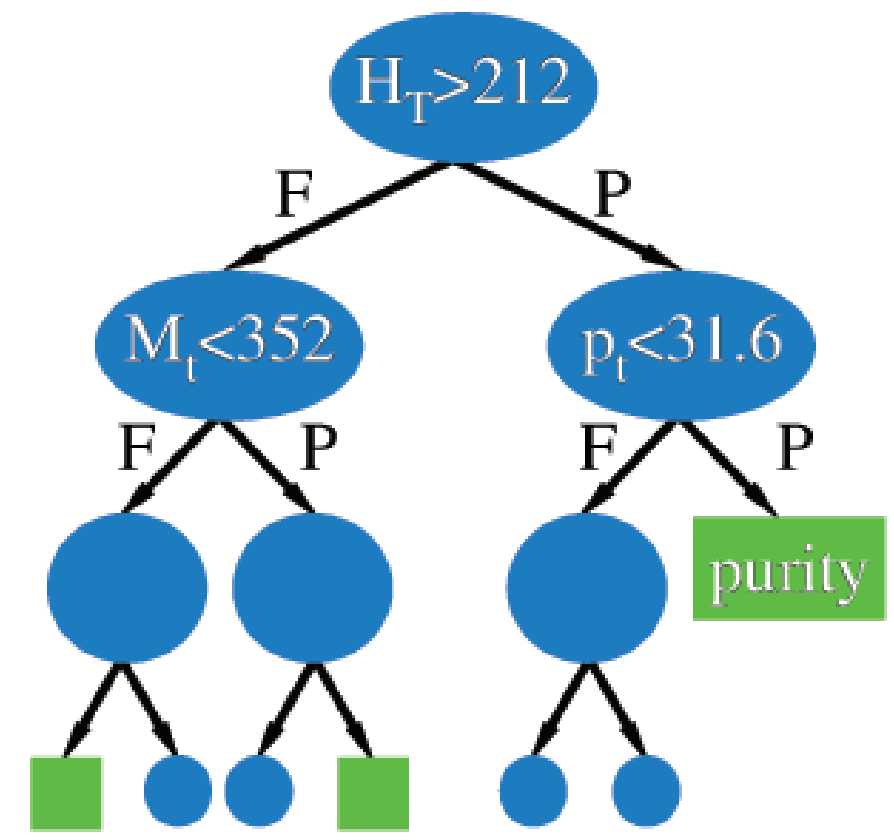

Figure 6.1: Schematic example of a decision tree. Nodes are shown in blue and terminal nodes (leaves) in green [51].

where $N_{s}\left(N_{b}\right)$ is the number of signal (background) events in the sample. The value of the cut is adjusted until the best purity is obtained, then another variable is considered and the procedure continues. This procedure continues iteratively to minimise the Gini factor, $G$ for the sum of passed and failed sides of a given cut, where the Gini Factor, $\mathrm{G}$ is defined as:

$$
G=P(1-P)
$$

A minimum value for this sum is set so that the sample is not over-trained. Over-training occurs when the decision tree becomes sensitive to the statistical fluctuations in the sample rather than the kinematics of the event. For a given tree this procedure continues until either a required number of leaves have been created or until each leaf is pure signal or pure background or too few events remain for further training.

Decision trees are improved by applying a boosting technique. Boosting has 
been shown to yield better results than neural networks when the number of input variables is high $(\sim 20)[52]$. This technique gives a higher weight to those events which are mis-classified, so they take higher priority in future iterations. For example, if a signal event fails the di-jet mass requirement in the first decision tree, it will gain a higher weight in the second decision tree. Here, due to the higher weight of this signal event, other variables may offer better discrimination and so a new tree structure will be created.

New trees are then created based upon the training sample with new weights derived from the previous tree, and the new decision tree construction begins. For this analysis 20 boosted trees are created as this is a good balance between a smooth BDT output distribution and computing time. A rating is assigned to variables based upon how often that variable appeared in each of the decision trees created, and also it's impact as a discriminating variable. This impact is assessed as the amount that the Gini Factor changed from the previous cut $\Delta G$. The variable rating shown in Table 6.1 represents the average over these trees.

\subsubsection{BDT Input Distributions}

The BDT is trained on the $W H \rightarrow l \nu b \bar{b}$ signal, exclusively for type 1 and type 2 tau candidate events in two and three jet bins for the one b-tag and two b-tag samples against all backgrounds (except the multi-jet background, because the low statistics here do not reflect the true shape of the sample). The training results are listed in Table 6.1, where a higher number indicates a more important variable in the BDT training. This table indicates that the di-jet mass offers the best discrimination between signal and background events as expected. However, other variables offer significant discrimination to improve the separation between signal and background over the di-jet mass distribution alone. This is particularly important for the events with three associated jets, as the background composi- 
tion is dominated by $t \bar{t}$ events, so variables like the transverse $W$ mass become more important than when trying to distinguish the signal from $W+$ jets events in two jet events.

\begin{tabular}{|c|c|c|c|c|c|c|c|c|}
\hline Variable & $1 \mathrm{bt} 1 \mathrm{j} 2$ & $1 \mathrm{bt} 1 \mathrm{j} 3$ & $1 \mathrm{bt} 2 \mathrm{j} 2$ & $1 \mathrm{bt} 2 \mathrm{j} 3$ & $2 \mathrm{bt} 1 \mathrm{j} 2$ & $2 \mathrm{bt} 1 \mathrm{j} 3$ & $2 \mathrm{bt} 2 \mathrm{j} 2$ & $2 \mathrm{bt} 2 \mathrm{j} 3$ \\
\hline$m_{j j}$ & 28.01 & 13.73 & 20.16 & 8.37 & 47.36 & 21.36 & 43.93 & 28.99 \\
\hline$H_{T}($ goodjets $)$ & 4.98 & 4.99 & 4.85 & 3.67 & 3.31 & 5.09 & 3.48 & 5.15 \\
\hline$\Delta \phi(j 1, j 2)$ & 1.50 & 9.69 & 3.16 & 5.21 & 2.16 & 4.64 & 4.84 & 9.24 \\
\hline$p_{T}(j 1)$ & 1.63 & 4.73 & 2.37 & 4.30 & 2.40 & 0.44 & 3.11 & 8.40 \\
\hline$p_{T}(j 2)$ & 2.70 & 9.77 & 2.86 & 6.59 & 1.82 & 14.07 & 3.93 & 7.33 \\
\hline$\eta(j 1)$ & 1.54 & 10.53 & 3.56 & 8.59 & 2.45 & 6.51 & 3.52 & 7.12 \\
\hline$\eta(j 2)$ & 1.71 & 9.06 & 3.21 & 7.50 & 1.16 & 6.45 & 3.45 & 8.93 \\
\hline$\left(H_{T^{-}} H_{\mathrm{T}}\right) /\left(H_{T}+H_{\mathrm{T}}\right)$ & 2.41 & 10.86 & 5.52 & 9.76 & 2.71 & 5.89 & 7.90 & 13.11 \\
\hline$H_{\mathrm{T}}$ & 2.30 & 10.33 & 2.29 & 5.43 & 2.61 & 3.19 & 2.82 & 6.92 \\
\hline$p_{T_{\text {trk }}}$ & 2.92 & 8.20 & 3.46 & 7.50 & 3.24 & 4.76 & 4.78 & 10.50 \\
\hline$p_{T}(\tau)$ & 2.46 & 7.61 & 3.61 & 7.30 & 3.25 & 7.20 & 3.11 & 8.21 \\
\hline$\Delta \phi\left(\tau, H_{\mathrm{T}}\right)$ & 4.02 & 4.06 & 4.21 & 5.59 & 4.68 & 5.34 & 4.85 & 7.08 \\
\hline$m_{T}(W)$ & 2.12 & 4.76 & 2.16 & 7.21 & 2.05 & 2.85 & 3.59 & 13.62 \\
\hline$H_{\mathrm{T}}(\mathrm{jets}+\mathrm{tau})$ & 3.86 & 6.72 & 2.50 & 6.19 & 2.85 & 2.04 & 3.01 & 8.20 \\
\hline$H_{T}(\mathrm{jets}+\mathrm{tau})$ & 3.12 & 3.86 & 3.62 & 4.49 & 2.48 & 0.95 & 5.27 & 6.08 \\
\hline
\end{tabular}

Table 6.1: Relative importance of different variables in the BDT averaged over all trees.

\subsubsection{BDT Output Distributions}

The BDT training uses one third of the total MC statistics, the remaining two thirds of the sample is used for the limit setting procedure. The BDT outputs for the eight input channels for a Higgs mass of $115 \mathrm{GeV}$ are shown in Figure 6.2. The agreement in the BDT output between background expectation and data is generally good in these distributions. The statistics are rather low in the type 1 tau event samples, especially with three associated jets. However, most sensitivity to the signal is in the type 2 tau events with two associated jets. The signal peaks towards high values of the BDT output, and the background towards lower values near zero. No significant excess is seen in the BDT output distributions, so limits on SM Higgs production are set. 


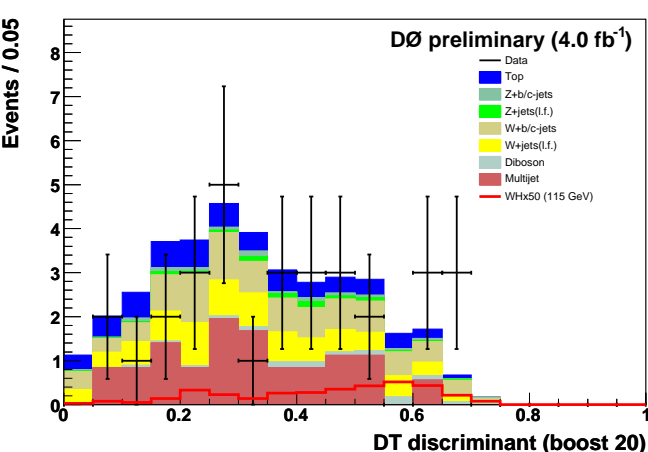

(a) BDT output, 1 btag, type 1, 2 jets

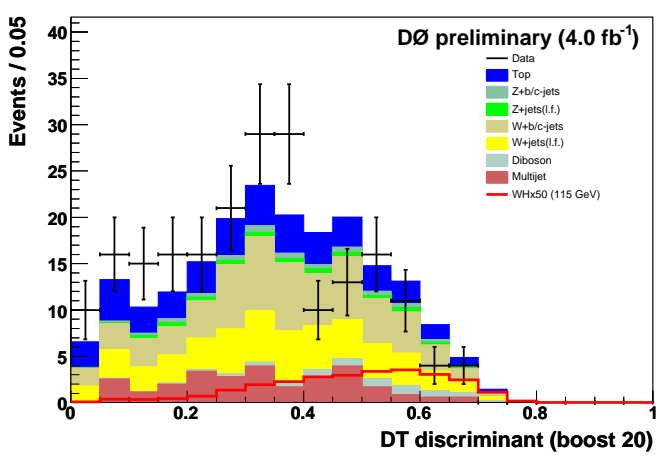

(c) BDT output, 1 btag, type 2, 2 jets

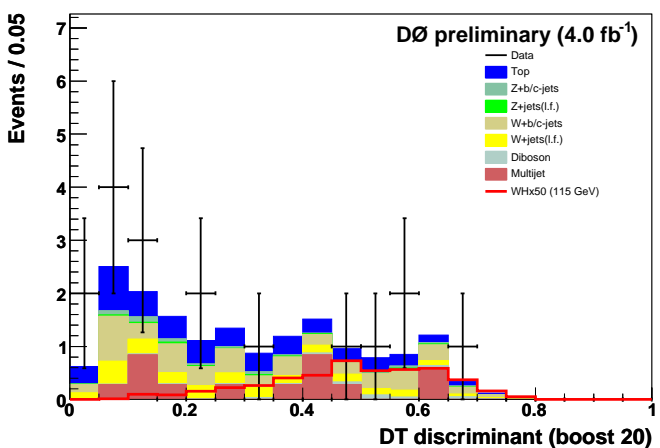

(e) BDT output, 2 btag, type 1, 2 jets

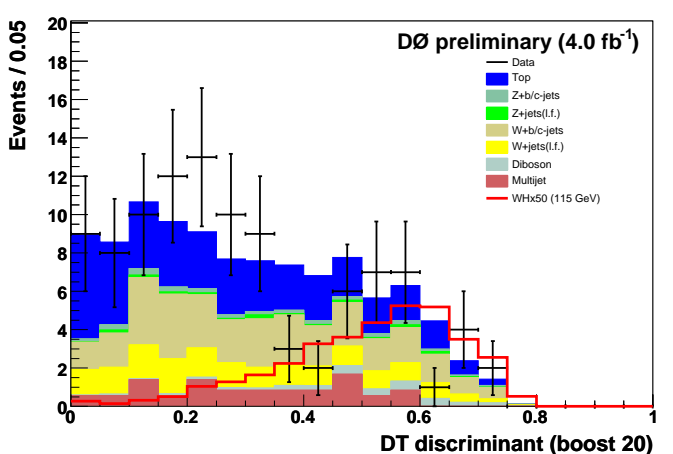

(g) BDT output, 2 btag, type 2, 2 jets

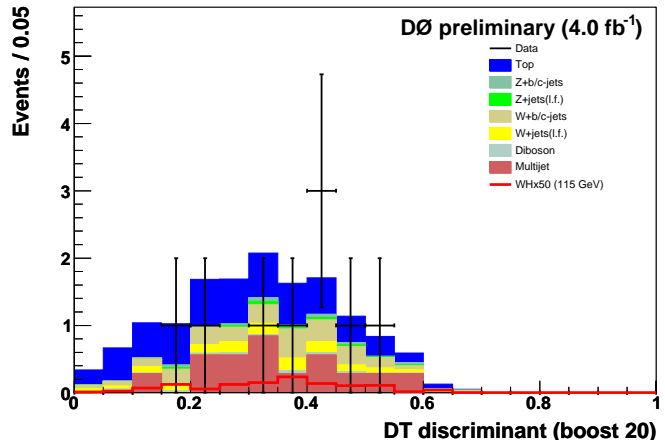

(b) BDT output, 1 btag, type 1, 3 jets

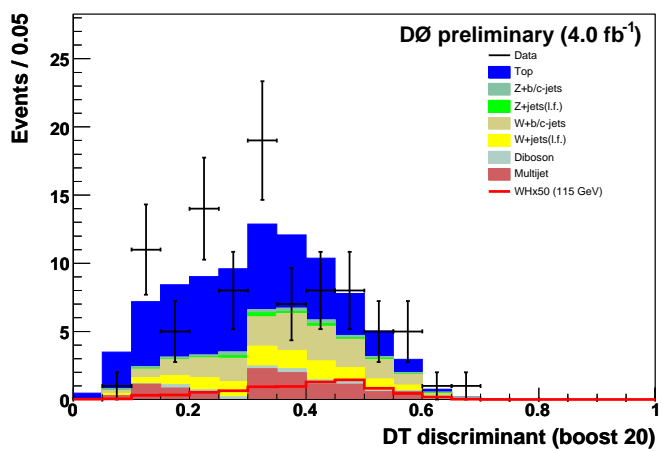

(d) BDT output, 1 btag, type 2, 3 jets

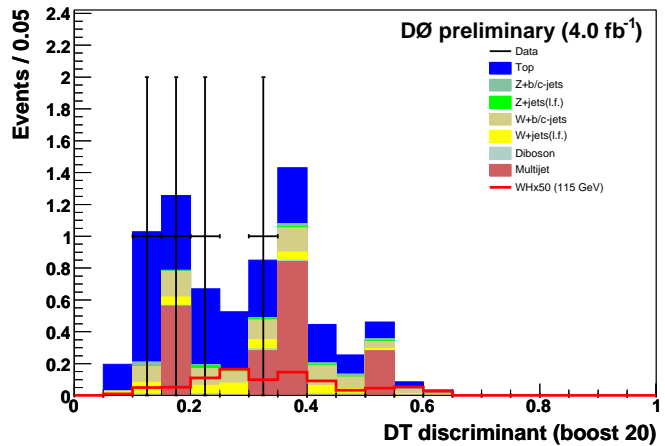

(f) BDT output, 2 btag, type 1, 3 jets

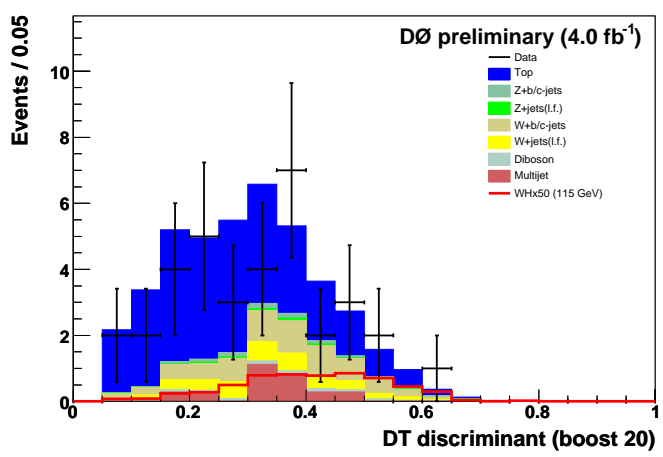

(h) BDT output, 2 btag, type 2, 3 jets

Figure 6.2: BDT outputs for each of the input channels to the limit setting procedure. Data are compared to the sum of expected backgrounds. 


\subsection{Systematic Uncertainties}

Various sources of systematic uncertainty affect the signal efficiency and the background estimations. It is crucial to understand the systematic uncertainties as they have a degrading effect on the analysis sensitivity.

Two types of systematic uncertainty are considered for this analysis. Systematic uncertainties which affect only the normalisation of the signal and/or background are known as Flat systematics. Systematic uncertainties which affect the boosted decision tree output in a differential manner are known as Shape systematics. The following sections describe individually the flat and shape systematics used in this analysis.

\subsubsection{Flat Systematics}

- The tau lepton identification uncertainty was calculated in a $Z \rightarrow \tau \tau$ sample and includes the uncertainty on the tau neural network and tau track efficiency. The uncertainty in efficiency for tau leptons was found to be $11 \%$ for type 1 taus and $4.5 \%$ for type 2 taus [43].

- The uncertainty on the taggability scale factors were derived by fluctuating the $\eta_{d}$ dependent uncertainty on the taggability and measuring the change in normalisation to the data [48]. The change was found to be $4.6 \%$.

- The uncertainty on the vertex confirmation scale factors is taken from the uncertainty of the fit for the scale factors in the appropriate $\eta_{d}$ range for jets in this analysis [47] and found to be $2 \%$.

- The uncertainty on the multi-jet background is estimated by considering the integrated number of events after all selections when applying the multi-jet reweighting and when the multi-jet reweighting is not applied (see Sec- 
tion 5.2.1). The uncertainty is found to be $12 \%$ for type 1 tau events and $7 \%$ for type 2 tau events. This is a small effect given the statistical uncertainty on the multi-jet estimate after all selections.

- The uncertainty on the diboson cross-section is $7 \%$ and is taken from the uncertainty on NLO QCD computations [53].

- The uncertainty on the single top cross-section is $10 \%$ [54].

- The uncertainty on the top pair cross-section is $10 \%$ and is taken from the following source on heavy quark pair production at two loops in QCD [55].

- The uncertainty on the $\mathrm{W} / \mathrm{Z}+$ light jets cross-section is $6 \%$ as recommended by the DØ Higgs group [56].

- The uncertainty on the heavy flavour scale factor applied to set the $W / Z+b \bar{b}$ and $W / Z+c \bar{c}$ contributions relative to $W / Z+j \bar{j}$ in the $W / Z+j e t s$ sample and taken from MCFM, is $+25 \% /-19 \%$ [40]. However, a value of $20 \%$ is taken in this analysis as an error on the light flavour cross-section (which is included in the uncertainty quoted from MCFM) is included separately.

- The uncertainty on the integrated luminosity is $6.1 \%$ [57].

- The uncertainty on the trigger parameterisation is taken from the $Z H \rightarrow$ $\nu \nu b \bar{b}$ study [49] and has a value of $5 \%$. However, an additional $2 \%$ uncertainty is added in quadrature to account for the assumption that the $p_{T}$ dependent turn on for taus is the same as the for jets in the parameterisation derived for this analysis. This yields a total uncertainty of $5.5 \%$ on all MC samples.

- The uncertainty on the parton distribution functions is considered by running each of the 20 pairs of reweightings associated with the incoming par- 
tons and taking the largest shape uncertainty to be the uncertainty on this quantity. Although a shape dependent uncertainty, the cumulative size of this uncertainty is found to be no greater than $3 \%$ for the signal MC samples [49] and so a flat uncertainty of this size is assigned.

\subsubsection{Shape Systematics}

Uncertainties on jet energy scale, jet energy resolution, jet identifcation efficiency and b-tagging scale factors and PDFs are all predicted to have an effect on the shape of the BDT output. Uncertainties such as the ALPGEN MLM and scale uncertainty only have a shape dependence (i.e. they preserve normalisation). The ALPGEN uncertainties are defined as follows:

- ALPGEN MLM/Scale uncertainty - The ALPGEN matching procedure allows the combination of matrix element calculations and parton showering without double counting. The uncertainty assigned here takes into account the differences in choice of renormalisation and factorisation $\alpha_{s}$ scales [59].

To derive the effect of shape dependent uncertainties the analysis was run again for each of the algorithms with \pm 1 standard deviation (s.d.) fluctuations and then the BDT output distribution is re-calculated. As the uncertainty on these quantities varies differentially with BDT output it is not possible to quote a single value, instead the differential uncertainty histograms are included in the limit setting procedure. An example of the differential uncertainty on $W+$ jets events arising from fluctuating the ALPGEN MLM uncertainty by +1 s.d. is shown in Figure 6.3. This distribution shows that assigning a flat systematic uncertainty to such a quantity would be incorrect. 


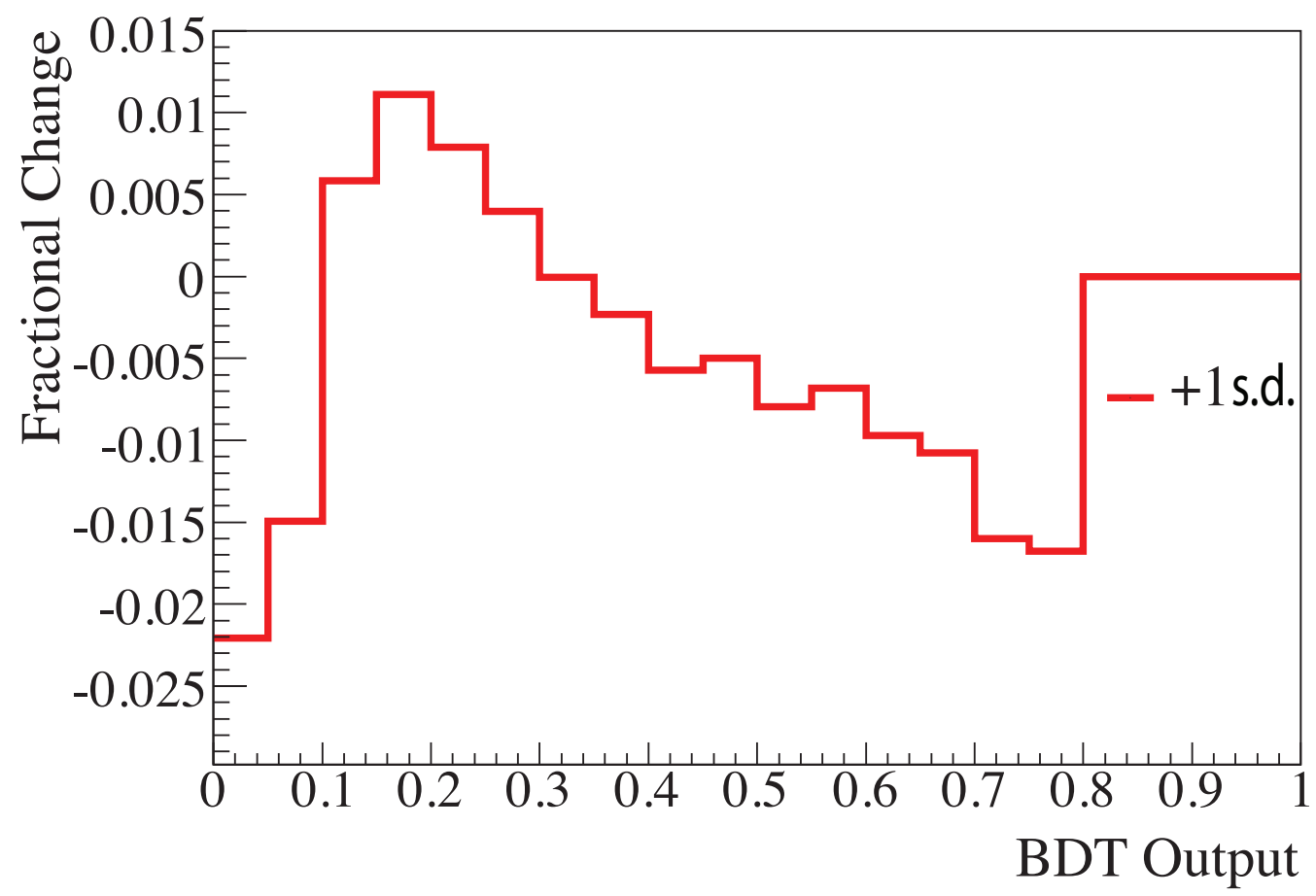

Figure 6.3: Differential uncertainty on $W+$ jet events from the ALPGEN MLM uncertainty with a +1 s.d. fluctuation. The fractional difference between the shifted result and the nominal result is plotted on the ordinate and the BDT output on the abscissa. 


\subsection{Limit Setting}

As no significant excess was observed in this analysis, limits are set on the production cross-section of a Higgs boson in the $W H \rightarrow \tau \nu b \bar{b}$ decay mode. This section describes the procedure used to set the limits, the treatment of the systematic uncertainties derived in the previous section and the results.

\subsubsection{Limit Setting Technique}

To quantify the sensitivity of this analysis to the Higgs signal, 95\% confidence level limits are set on the production cross-section for associated Higgs boson production. The method used is based on the "LEP $C L_{s}$ method" [60, 61]. This method uses modified Frequentist confidence levels and a log-likelihood ratio (LLR) test statistic. Two hypotheses are considered for limit setting: The background only and the signal plus background hypotheses. The background only hypothesis is based upon the predicted background only. The signal plus background hypothesis is based upon the sum of the predicted background and the expected Higgs boson signal contribution.

The LLR is defined as:

$$
L L R=-2 \sum s_{i}-n_{i} \ln \left(1+\frac{s_{i}}{b_{i}}\right)
$$

where $i$ indicates the $i^{\text {th }}$ bin in the discriminating distribution used to set the limits, and $s_{i}, b_{i}$ and $n_{i}$ are the number of signal, background and data entries in the $i^{\text {th }}$ bin respectively. The ratio of the LLRs in the signal plus background and background only hypotheses gives best results for searches with small statistics [62], which is the case after applying b-tagging cuts in this analysis. The expectations for the LLR are calculated over a large number of pseudo-experiments. The pseudo-data are generated by picking a random number from a Poisson dis- 
tribution, where the probability of obtaining $x$ events given an expectation of $p$ events is given by:

$$
P(x ; p)=\frac{p^{x} e^{-p}}{x !}
$$

The consistency of the observed data with the background and signal plus background hypotheses yields an observed limit. The sensitivity to the signal is defined by assuming the observation is exactly that predicted by the background model; this is the expected limit.

The confidence level in the signal plus background hypothesis is determined by the probability that the LLR in the signal plus background hypothesis, $\mathrm{LLR}_{s+b}$ is less than the LLR of the data, $\mathrm{LLR}_{d}$. The confidence level in the background only hypothesis is determined from the probability that the LLR in the background only hypothesis is less than $\mathrm{LLR}_{d}$. From these two confidence levels the confidence level in the signal, $\mathrm{CL}_{s}$ can be calculated:

$$
C L_{s}=\frac{C L_{s+b}}{C L_{b}}=\frac{P_{s+b}\left(L L R \leq L L R_{d}\right)}{P_{b}\left(L L R \leq L L R_{d}\right)}
$$

where $P_{s+b}\left(P_{b}\right)$ is the probability of obtaining such a LLR in the signal plus background (background) hypothesis. A 95\% confidence level exclusion is set when $1-C L_{s}>0.95$.

Systematic uncertainties have a degrading effect on the limits as they create a larger range of values for the pseudo-data, which widens the LLR distribution. These uncertainties are taken into account by varying each pseudo-experiments expectation by a Gaussian of mean zero and width equal to one. Quantitatively, the pseudo-experiments prediction is varied in the following way:

$$
p_{i}^{\prime}=p_{i}^{0}\left(1+\sum_{k} \sigma_{k}^{i} S_{k}\right)
$$


where $p_{i}^{\prime}\left(p_{i}^{0}\right)$ represent the new (nominal) prediction, $\sigma_{k}^{i}$ represents the $k^{\text {th }}$ systematic uncertainty in the $i^{t h}$ bin and $S_{k}$ represents the Gaussian random number for that particular systematic uncertainty. This procedure will result in a widening of the LLR distribution. More details on the limit setting procedure can be found in [62].

\subsubsection{Fitting Uncertainties}

The degrading effect of systematic uncertainties can be minimised by performing a fit to the data and background model prediction. This procedure varies the default hypothesis such that the probability distribution functions are maximised over all possible values of the uncertainties. A fit calculates the optimum central value for a given systematic uncertainty, while retaining a term to sum the square deviation of the systematic in the following way:

$$
\chi^{2}=2 \sum\left(\left(p_{i}^{\prime}-d_{i}\right)-d_{i} \ln \frac{p_{i}^{\prime}}{d_{i}}\right)+\sum_{k} S_{k}^{2},
$$

where $p_{i}^{\prime}=\Pi_{k}\left(1+S_{k}\right)$. $p_{i}$ is the sum of signal plus background in the $i^{t h}$ bin for the signal plus background hypothesis and is the background in the $i^{\text {th }}$ bin for the background only hypothesis. New values for the signal and background expectations in each bin are then calculated as follows:

$$
b_{i}^{\prime}=b_{i} \Pi_{k}\left(1+S_{k}\right), s_{i}^{\prime}=s_{i} \Pi_{k}\left(1+S_{k}\right),
$$

where $b_{i}^{\prime}\left(s_{i}^{\prime}\right)$ are the new background(signal) expectations in the $i^{\text {th }}$ bin. These new expectations are used in the calculation of a redefined LLR as follows:

$$
L L R=-\log \left(\frac{\chi_{\min }^{2}(\text { background only })}{\chi_{\min }^{2}(\text { signal plus background })}\right) .
$$


This procedure can significantly change the width of the LLR distribution for an analysis and so the amount that the central value of each systematic uncertainty is varied by under fitting is checked to make sure sensible values are observed. More details on the fitting procedure can be found in [63]. Figure 6.4 shows the variation of the central value of systematic uncertainties in this analysis after fitting is performed. No systematic uncertainty is found to fluctuate more than \pm 0.6 s.d.

\section{Best Fit to Data Parameters}

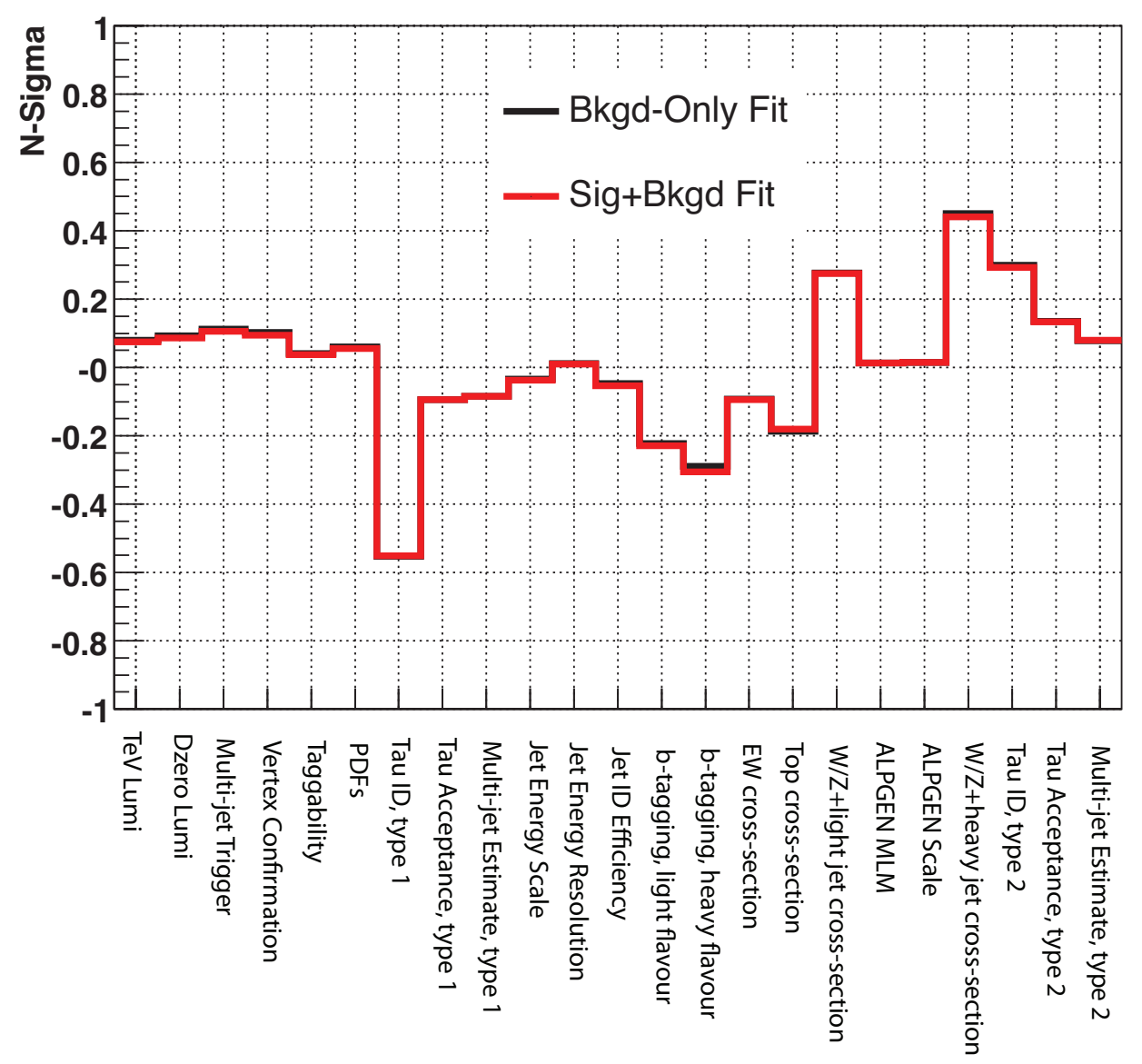

Figure 6.4: Variation in standard deviations of each systematic uncertainty after fitting the background only (black) and signal plus background (red) hypotheses.

The range of values achieved across the pseudo-experiements for the total uncertainty on the signal and background is more constrained than before the 
fitting procedure. Distributions of the no-fit, background only fit and signal plus background fits are shown for the signal and background in Figure 6.5. These distributions show the value of the total systematic uncertainty for each pseudo-experiment, where a value of one is the nominal uncertainty before the Gaussian smearing effects are applied. Comparing the signal and background uncertainty distributions after fitting shows that the background uncertainty is more constrained by the fitting than the signal uncertainty. This is expected as the total background systematic is larger than that of the signal due to the heavy flavour uncertainty for example.

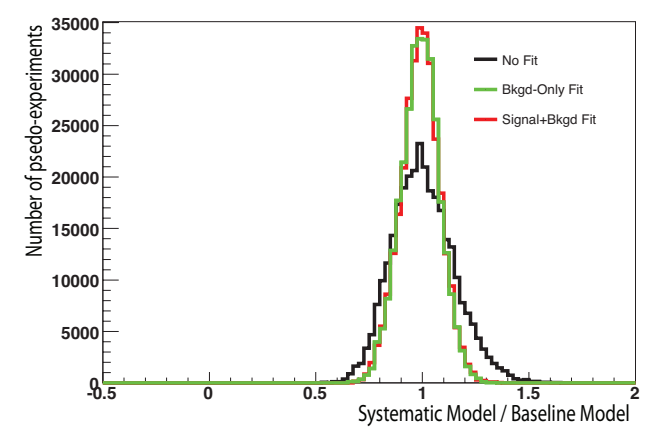

(a) Signal

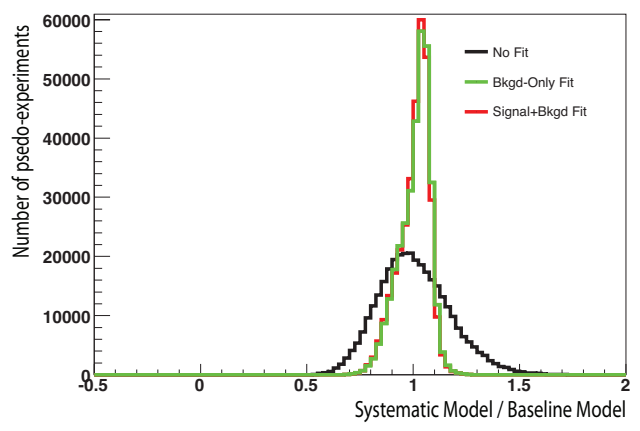

(b) Background

Figure 6.5: Fitted and Gaussian (no-fit) (a) signal and (b) background systematic distributions for the signal plus background and background only hypotheses.

\subsubsection{Results}

As the integrated number of events observed in data agrees within uncertainties with the expected background, limits for the cross-section times branching ratio for the Higgs signal are set. The signal processes $W H \rightarrow l \nu b \bar{b}$ and $Z H \rightarrow l \bar{l} b \bar{b}$ are added and limits are set using the collie package v00-03-17 [64]. Initially, limits are set with Gaussian systematic uncertainties, then limits are derived using the fitted systematic uncertainties as described in Section 6.3.2. Expected 
limits are also extracted using no systematic uncertainties in order to understand the degrading effect of the systematics.

For the limit setting eight input channels are used: type 1 and type 2 tau candidate events with both two and three jets in the event with one or two btagged jets. The BDT output distribution is used as the input statistic for the limit setting procedure.

The limits obtained for the $W H \rightarrow \ell \nu b \bar{b}$ and $Z H \rightarrow \ell \bar{\ell} b \bar{b}$ processes relative to the SM at the $95 \%$ confidence level are shown in Table 6.2 and Figure 6.6 at mass points from $100 \mathrm{GeV}$ to $150 \mathrm{GeV}$ in $5 \mathrm{GeV}$ steps. The log likelihood ratio is plotted against the Higgs mass with \pm 1 s.d. bands in Figure 6.7.

The observed limits are better than the expected limits, which is consistent with the fact that a slight data deficit is observed compared to the predicted background, most notably in the di-jet mass distribution (which is the most significant variable in the BDT - see Table 6.1) between 100-150 GeV in the 3-jet channels (see Figure A.13(a)). The limits using the fitted systematics approach

\begin{tabular}{|c|c|c|c|}
\hline & Exp & $\operatorname{Exp}($ Obs $)$ & $\operatorname{Exp}($ Obs $)$ \\
\hline$m_{H}(\mathrm{GeV})$ & No Systematics & Gaussian Systematics & Fitted Systematics \\
\hline 100 & 10.7 & $24.9(22.7)$ & $13.2(9.1)$ \\
\hline 105 & 11.7 & $27.2(24.8)$ & $14.8(9.7)$ \\
\hline 110 & 13.7 & $33.2(30.5)$ & $17.7(11.9)$ \\
\hline 115 & 16.8 & $42.4(40.4)$ & $22.4(14.1)$ \\
\hline 120 & 20.8 & $56.0(52.0)$ & $29.6(17.8)$ \\
\hline 125 & 27.2 & $27.3(24.5)$ & $42.8(24.6)$ \\
\hline 130 & 36.7 & $110(113)$ & $61.2(40.6)$ \\
\hline 135 & 52.8 & $170(176)$ & $105(69.1)$ \\
\hline 140 & 76.6 & $247(276)$ & $161(140)$ \\
\hline 145 & 114 & $370(436)$ & $247(290)$ \\
\hline 150 & 184 & $608(716)$ & $406(557)$ \\
\hline
\end{tabular}

Table 6.2: Expected and observed limits for no systematics, Gaussian systematics and fitted systematics methods.

are shown in Figure 6.6. 


\section{$D \varnothing$ preliminary, $4.0 \mathrm{fb}^{-1}$}

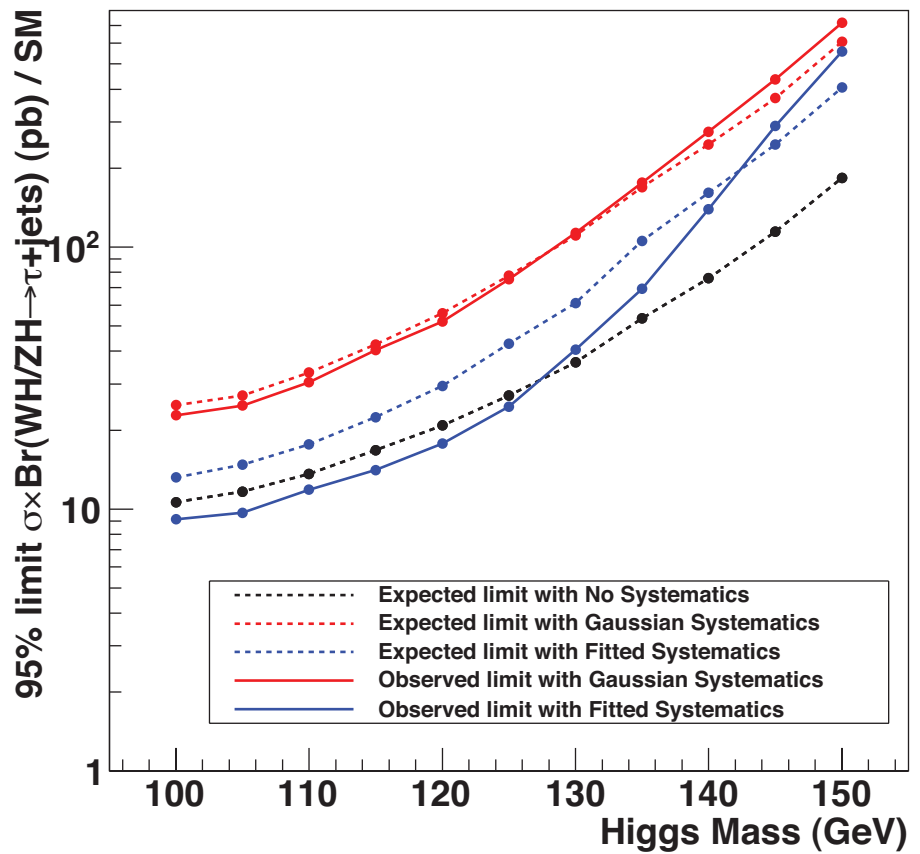

Figure 6.6: Limits obtained for the $W H / Z H$ process relative to the $\mathrm{SM}$ cross-section at the 95\% confidence level as a function of the Higgs mass. The dashed lines indicate the expected limits and the solid lines indicate the observed limits. The black line assumes no systematic uncertainties, the red lines assume Gaussian systematics and the blue lines assume fitted systematic uncertainties.

The LLR distribution associated with the fitted systematics limits is shown in Figure 6.7 for the background only hypothesis and the signal plus background hypothesis. The separation of these two LLR values relative to the \pm 1 s.d. and \pm 2 s.d. banded regions show the sensitivity of the experiment to the signal. These bands show the the width of the LLR distribution in the background only case, including the effect of systematic uncertainties and indicate how sensitive the analysis is to a fluctuation in the data. This analysis sets limits on cross-sections an order of magnitude larger than those predicted by the SM for associated Higgs boson production and the separation of the LLR values is therefore smaller than the bands. The difference between the LLR in the background only and signal plus background hypotheses spans around 0.1 at $100 \mathrm{GeV}$, yet the \pm 1 s.d. band 
spans almost 0.8 . The observed LLR for data is also shown. A value below zero would indicate a signal-like fluctuation of the data.

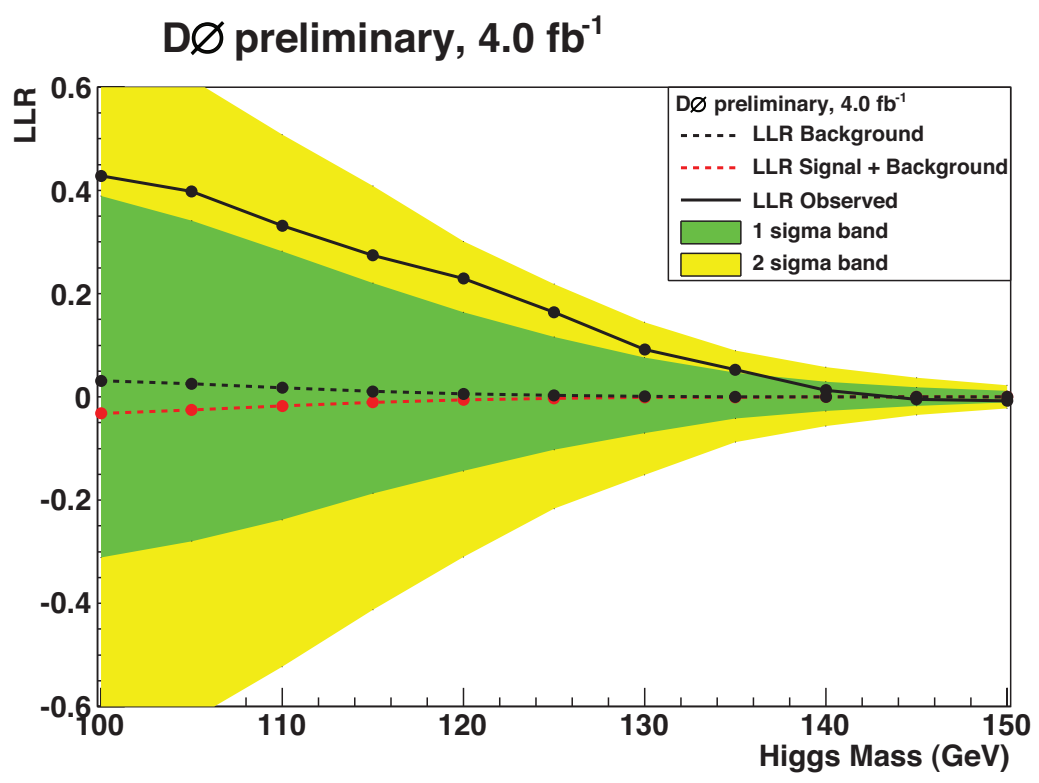

Figure 6.7: Log Likelihood Ratio as a function of Higgs boson mass using the fitted systematics method. 


\section{Chapter 7}

\section{Discussion of Results}

\subsection{Comparison with other channels}

The aim of this section is to put the results described in the previous section into context by comparing them to other channels in the latest $\mathrm{D} \varnothing$ combination. Table 7.1 shows the important input channels at low mass for the latest $D \varnothing$ combination, for which the $4 \mathrm{fb}^{-1} W H \rightarrow \tau \nu b \bar{b}$ result was not available and so the $1 \mathrm{fb}^{-1}$ result is still included.

\begin{tabular}{|c||c||c|c||c|}
\hline Channel & Luminosity & Observed Limit & Expected Limit & Expected Limit Scaled to $5.1 \mathrm{fb}^{-1}$ \\
\hline$W H \rightarrow(e / \mu) \nu b b$ & $5.1 \mathrm{fb}^{-1}$ & 6.9 & 5.1 & 5.1 \\
\hline$W H \rightarrow \tau \nu b b$ & $0.9 \mathrm{fb}^{-1}$ & 35 & 42 & 17.6 \\
\hline$(W / Z) H \rightarrow \tau \tau q \bar{q}$ & $4.9 \mathrm{fb}^{-1}$ & 27.0 & 15.9 & 15.6 \\
\hline$Z H \rightarrow \nu \nu b b$ & $2.1 \mathrm{fb}^{-1}$ & 7.5 & 8.4 & 5.4 \\
\hline$Z H \rightarrow l l b b$ & $4.1 \mathrm{fb}^{-1}$ & 9.1 & 8.0 & 7.2 \\
\hline$H \rightarrow \gamma \gamma$ & $4.2 \mathrm{fb}^{-1}$ & 24.3 & 33.0 & 30.0 \\
\hline$t \bar{t} H$ & $2.1 \mathrm{fb}^{-1}$ & 45 & 64 & 41.1 \\
\hline \hline$W H \rightarrow \tau \nu b b$ & $4.0 \mathrm{fb}^{-1}$ & 14.1 & 22.4 & 19.6 \\
\hline
\end{tabular}

Table 7.1: Important channels at low mass for the $\mathrm{D} \varnothing$ combination. Limits are shown for a Higgs mass of $115 \mathrm{GeV}[65]$.

A direct comparison of the limits does not give a fair reflection of the sensitivity of the individual channels without taking into account the luminosity of the data sample used. As the limits improve statistically with the square root 
of the integrated luminosity increase, it is possible to scale each of these results to compare each of the channels on an equal footing. After a scaling assuming no improvement in the systematic uncertainties, the results for the $W H \rightarrow \ell \nu b \bar{b}$ and $Z H \rightarrow \nu \nu b \bar{b}$ channels are shown to be the most powerful search channels for a low mass Higgs boson at D $\varnothing$.

Although the $W H \rightarrow(e / \mu) \nu b \bar{b}$ channel has the best sensitivity currently, the $Z H \rightarrow \nu \nu b \bar{b}$ channel is also very sensitive. This is mainly due to gaining additional sensitivity from the $W H \rightarrow l \nu b \bar{b}$ signal, where the lepton is not reconstructed and the large $Z \rightarrow \nu \nu$ branching fraction. As the production crosssection for the $W H$ signal is higher than that of the $Z H$ signal (Figure 2.3), this additional signal makes up almost half of the total signal in the $Z H \rightarrow \nu \nu b \bar{b}$ search.

Direct comparison of the $4 \mathrm{fb}^{-1} W H \rightarrow \tau \nu b \bar{b}$ result to the $5.1 \mathrm{fb}^{-1} W H \rightarrow$ $(e / \mu) \nu b \bar{b}$ shows that the electron and muon channels are more sensitive than the hadronic tau channel. This is expected as the efficiency for reconstructing electrons and muons is significantly higher than the efficiency for reconstructing a hadronic tau decay. Additionally, the $W H \rightarrow(e / \mu) \nu b \bar{b}$ channel gains sensitivity where the associated $W$ decays to $\tau \nu$ and the $\tau$ decays leptonically. However, the limits for the $W H \rightarrow \tau \nu b \bar{b}$ are only around a factor of three less than the $W H \rightarrow(e / \mu) \nu b \bar{b}$. This is equivalent to an increase in integrated luminosity for the combination of these two channels of around $10 \%$.

As a comparison to the $(W / Z) H \rightarrow \tau \tau q \bar{q}$ analysis, the expected limits for the $W H \rightarrow \tau \nu b \bar{b}$ are better, but the observed limits are similar, predominantly due to a data deficit observed in the final sample in $(W / Z) H \rightarrow \tau \tau q \bar{q}$. However, both of these analyses have shown that additional sensitivity to Higgs boson searches at $\mathrm{D} \varnothing$ and the Tevatron can be gained via the use of hadronic tau decay channels.

One of the benefits of performing a combination of results, either at $\mathrm{D} \varnothing$ or as 
part of the Tevatron combination, is that many of the systematic uncertainties, which have a degrading effect on the limit, (see Section 6.3.1) can be correlated. Many of the analyses searching for a low mass Higgs boson at the Tevatron will be dominated by similar uncertainties to the analysis presented in this thesis, such as the heavy flavour uncertainty and cross-section uncertainties. By correlating these uncertainties between channels in the fitting (see Section 6.3.2) further constraints are placed on the total systematic uncertainty, so the final limit is degraded less. Table 7.2 [65] shows the systematic uncertainties for the low mass channels in the DØ SM Higgs combination, uncertainties with the same name are correlated, even if their value differs between analyses.

Figure 7.1 [65] shows the summer 2009 D $\varnothing$ SM Higgs combination which includes the channels whose systematic uncertainties are described in Table 7.2 [65], including the $1 \mathrm{fb}^{-1} W H \rightarrow \tau \nu b \bar{b}$ result. This figure shows that the $\mathrm{D} \varnothing$ experiment is less than a factor of two away from excluding a Higgs boson of mass $m_{H}=$ $\sim 165 \mathrm{GeV}$ and around a factor of 2.5 away from excluding a Higgs boson of mass $m_{H}=115 \mathrm{GeV}$. Only by including all possible search channels like $W H \rightarrow \tau \nu b \bar{b}$ will exclusion of a low mass Higgs boson be possible before the end of running.

\subsection{Projections}

A number of channels in the current Tevatron combination are not using the full dataset and other channels are being studied for inclusion before the end of Tevatron running. Projections have been made by D $\varnothing$ and CDF showing how the limits on Higgs boson production will look with increasing integrated luminosity.

Figure $7.2[66]$ shows a projection by the CDF collaboration which assumes $\mathrm{CDF}$ and $\mathrm{D} \varnothing$ achieve the same sensitivity to a SM Higgs boson of mass $m_{H}=$ $115 \mathrm{GeV}$. The lines indicate the statistical extrapolation of the results achieved 


\begin{tabular}{|c|c|c|c|c|}
\hline Source & $W H \rightarrow e \nu b \bar{b}$ & $W H \rightarrow \mu \nu b \bar{b}$ & $W H \rightarrow \tau \nu b \bar{b}\left(1 \mathrm{fb}^{-1}\right)$ & $W H \rightarrow W W^{+} W^{-}$ \\
\hline Luminosity/Normalisation & 6.1 & 6.1 & 6.1 & 6.1 \\
\hline Jet Energy Scale & 3.0 & 3.0 & 3.0 & - \\
\hline Jet ID & 5.0 & 5.0 & 4.0 & - \\
\hline Jet Triggers & - & - & 5.5 & - \\
\hline Tau Energy Scale/ID & - & - & 7.0 & - \\
\hline Electron ID/Trigger & 4.0 & - & - & 11 \\
\hline Muon ID/Trigger & - & 5.0 & - & 11 \\
\hline$b$-Jet Tagging & $3-6$ & $3-6$ & $4-6$ & - \\
\hline Background $\sigma$ & $6-20$ & $6-20$ & $6-18$ & $6-18$ \\
\hline Multijet & 14 & 14 & 25 & $30-50$ \\
\hline Shape Dependent Bkgd Modelling & $2-10$ & $2-10$ & $5-20$ & - \\
\hline Source & $Z H \rightarrow \nu \nu b \bar{b}$ & $Z H \rightarrow e^{+} e^{-} b \bar{b}$ & $Z H \rightarrow \mu^{+} \mu^{-}$ & \\
\hline Luminosity/Normalisation & 6.1 & 6.1 & 6.1 & \\
\hline Jet Energy Scale & 3.0 & 2.0 & 2.0 & \\
\hline Jet ID & 2.0 & 5.0 & 5.0 & \\
\hline Jet Triggers & 5.5 & - & - & \\
\hline Electron ID/Triggers & - & 4.0 & - & \\
\hline Muon ID/Triggers & - & - & 4.0 & \\
\hline$b$-Jet Tagging & 6.0 & $3.0-7.5$ & $3.0-7.5$ & \\
\hline Background $\sigma$ & $6-16$ & $10-30$ & $10-30$ & \\
\hline Multijet & 50 & $41-50$ & 50 & \\
\hline Shape Dependent Bkgd Modelling & - & $5-10$ & $5-10$ & \\
\hline Source & $H \rightarrow W^{+} W^{-}$ & $t \bar{t} H \rightarrow t \bar{t} b \bar{b}$ & $H \rightarrow \gamma \gamma$ & $H+X \rightarrow \tau \tau b \bar{b} / q \bar{q} \tau \tau$ \\
\hline Luminosity/Normalisation & $4-6$ & 6.1 & 6.1 & 6.1 \\
\hline Jet Energy Scale & 3.0 & - & - & 7.5 \\
\hline Jet ID & $1-2$ & - & - & 6 \\
\hline Tau Energy Scale/ID & - & - & - & 9 \\
\hline Electron ID/Trigger & $3-10$ & 2.5 & 3 & - \\
\hline Muon ID/Trigger & $7.7-10$ & 2 & - & 7 \\
\hline$b$-Jet Tagging & - & - & - & - \\
\hline Background $\sigma$ & $6-20$ & $10-15$ & 6 & $6-20$ \\
\hline Signal $\sigma$ & 10 & - & 10 & 10 \\
\hline Multijet & $5-20$ & $1-5$ & 1 & $5-40$ \\
\hline Shape Dependent Bkgd Modelling & $5-20$ & - & $5-7$ & - \\
\hline
\end{tabular}

Table 7.2: Systematic uncertainties for channels used in the $\mathrm{D} \varnothing$ combination. Uncertainties with the same name are correlated across channels [65].

at the dates given. The band shows the range of possible sensitivities based upon predicted improvements. These improvements include the addition of extra channels, improvement in object identification, triggering and analysis technique amongst others. This figure shows that exclusion of a Higgs boson of mass $m_{H}=$ $115 \mathrm{GeV}$ should be achievable when both experiments have analysed between 5.5 $-11 \mathrm{fb}^{-1}$ of data in all channels.

Figure 7.3 [67] shows the predicted sensitivity of the ATLAS experiment [68] at the LHC [69]. The ordinate on the left side shows the integrated luminosity and on the right side the scale indicates the confidence level for excluding a Higgs 


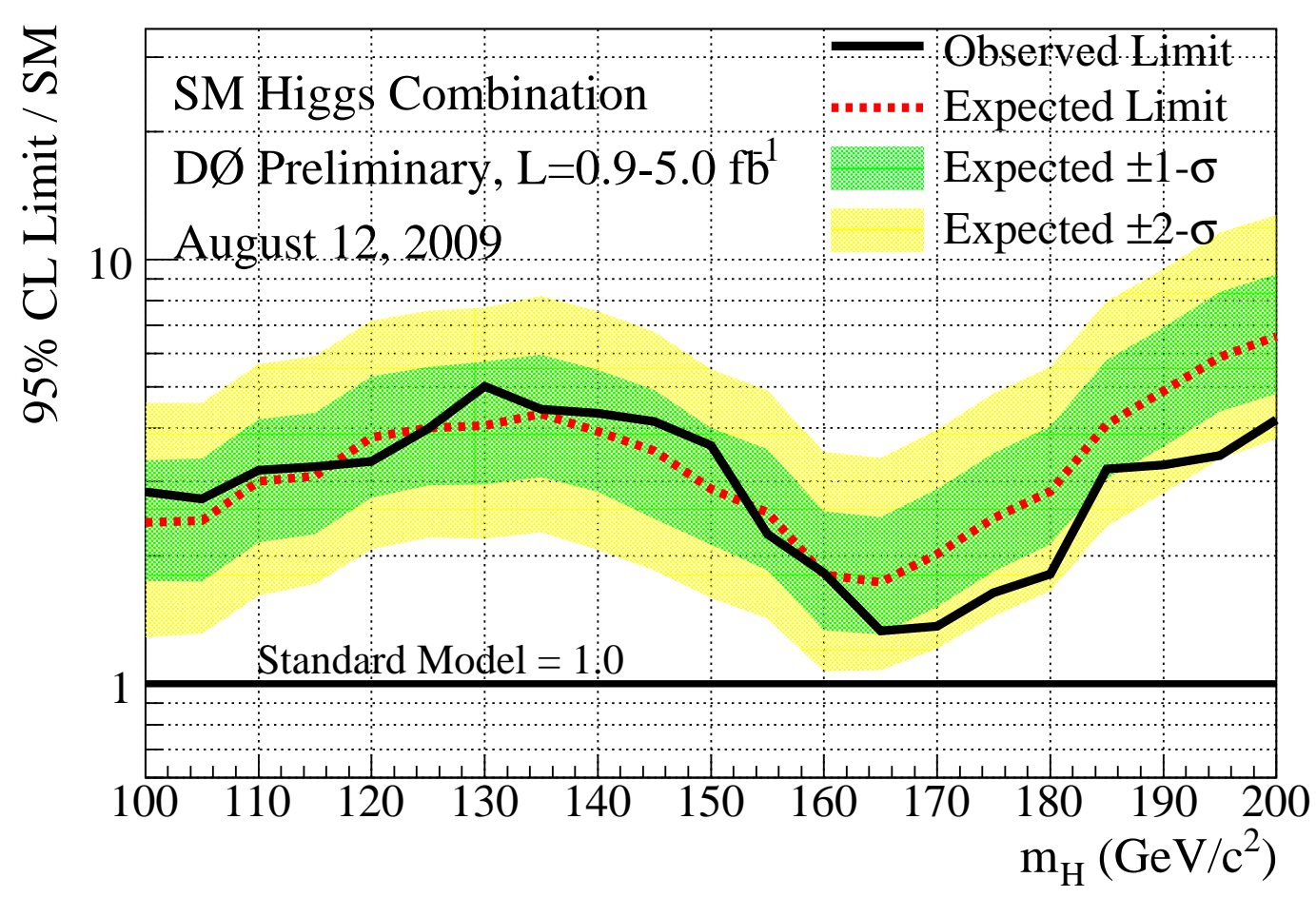

Figure 7.1: Summer 2009 DØ SM Higgs combination limits [65], this includes the $1 \mathrm{fb}^{-1}$ version of the $W H \rightarrow \tau \nu b \bar{b}$ analysis.

boson, whose mass is on the abscissa. ATLAS requires at least $2 \mathrm{fb}^{-1}$ of data to exclude a Higgs boson of mass $m_{H}=115 \mathrm{GeV}$. This study is made assuming $p p$ collisions at the centre-of-mass energy $\sqrt{s}=14 \mathrm{TeV}$. However, at the time of writing the LHC is likely to have collisions at around $7 \mathrm{TeV}[70]$ which reduces the sensitivity to a low mass Higgs boson significantly. This, coupled with the fact that ATLAS is expected to collect less than $1 \mathrm{fb}^{-1}$ by the end of 2010 , means that the Tevatron will be at the frontier of SM Higgs searches for at least another few years.

\subsection{Comparison with the Previous Analysis}

This section compares the result of this thesis to the previous version of the analysis, also performed by the author using $1 \mathrm{fb}^{-1}$ of data. A brief review of the 


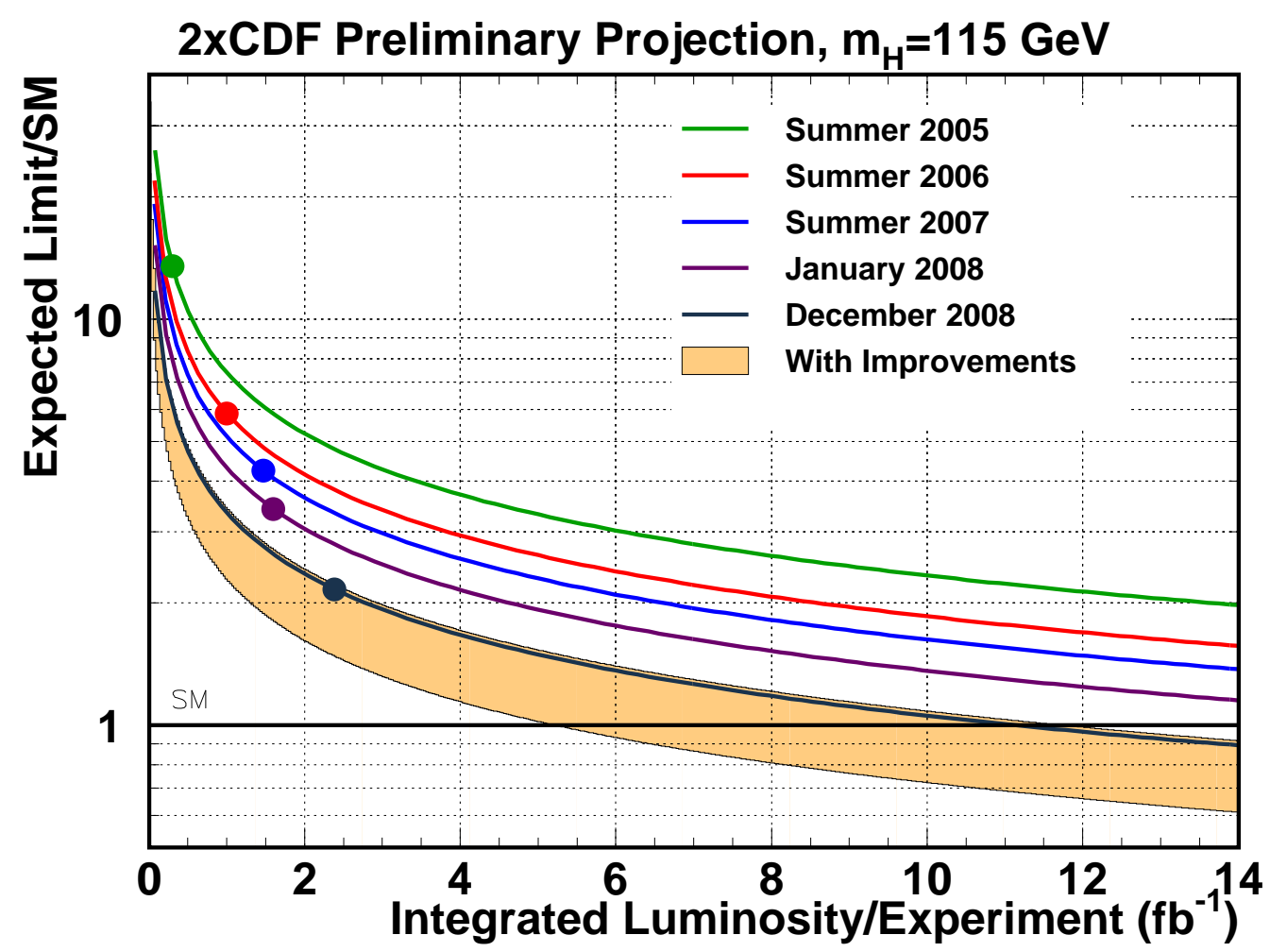

Figure 7.2: Projection for SM Higgs searches at the Tevatron for a Higgs boson of mass $m_{H}=$ $115 \mathrm{GeV}[66]$.

previous result, followed by the improvements made in the latest result and then an outlook of future versions of the $W H \rightarrow \tau \nu b \bar{b}$ analysis is presented.

\subsubsection{Summary of $1 \mathrm{fb}^{-1}$ Result}

The main differences between the $1 \mathrm{fb}^{-1}$ analysis and the result presented in this thesis are: the way the multi-jet background was removed and the final classifier used for the limit setting. As the di-jet mass distribution was used to set limits in the $1 \mathrm{fb}^{-1}$ result, it was optimal to obtain the purest possible sample with cuts. The $\mathbb{E}_{\mathrm{T}}$ requirement at the preselection stage was higher; $\mathbb{E}_{\mathrm{T}}>30 \mathrm{GeV}$ for the $1 \mathrm{fb}^{-1}$ version compared to $\mathscr{E}_{\mathrm{T}}>15 \mathrm{GeV}$ for the result presented in 


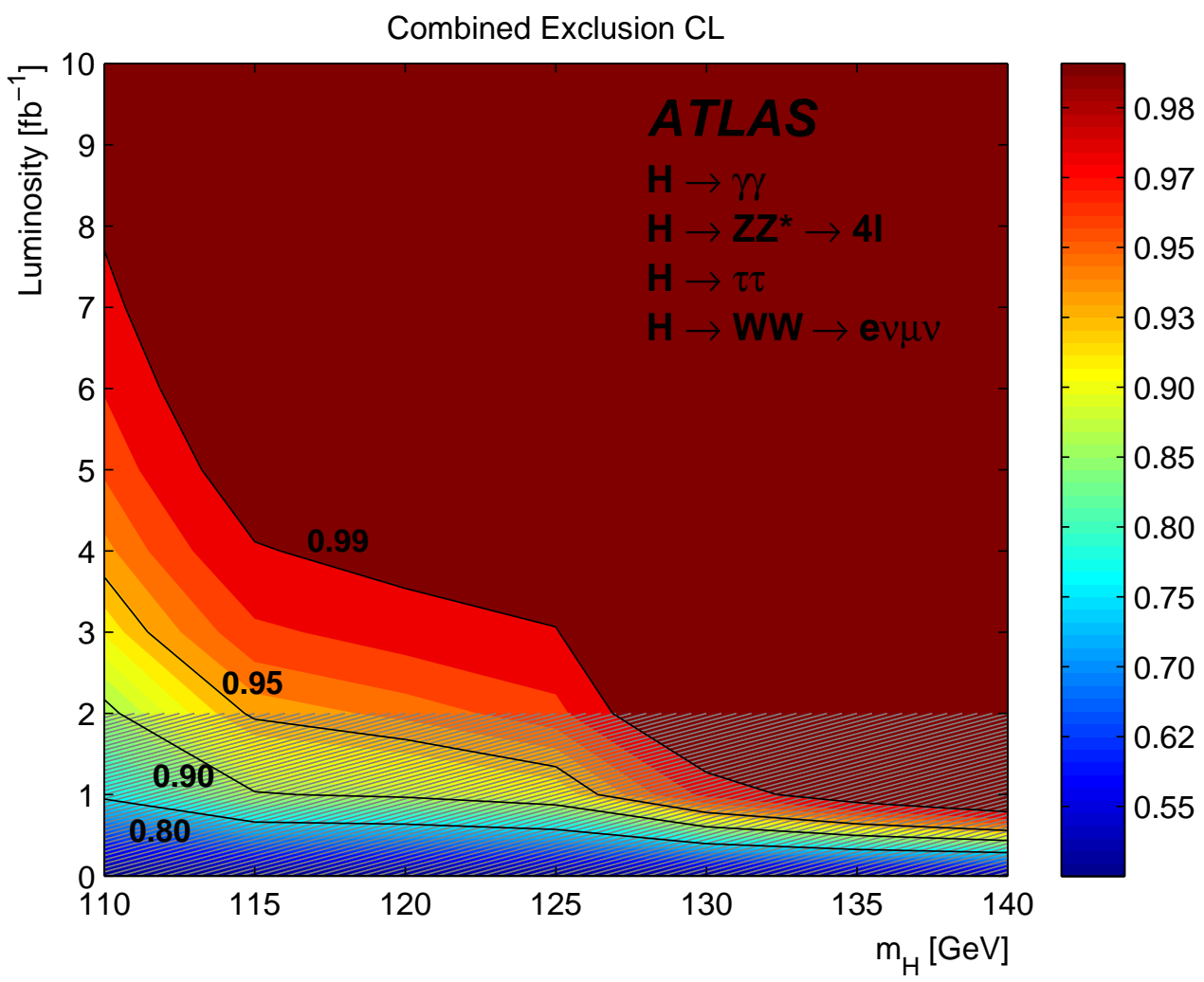

Figure 7.3: Predicted sensitivity to a SM Higgs boson at the ATLAS experiment [67].

this thesis. The majority of the multi-jet background was removed by a twodimensional triangle cut on the azimuth between the tau candidate and the $\mathbb{E}_{\mathrm{T}}$ and the $E_{\mathrm{T}}$ itself. Figure 7.4 shows the distribution of signal events and multi-jet events in this variable and the location of the cut, optimised to remove most of the multi-jet background and preserve most of the signal.

After the triangle cut, the sample was dominated by $W+$ jets events. The transverse momentum of the tau candidate and the transverse W mass are shown in Figure 7.5 to demonstrate the good agreement between the data and the predicted background.

Another difference between the $1 \mathrm{fb}^{-1}$ analysis and the result presented in this thesis is in the b-tagging configuration. The $1 \mathrm{fb}^{-1}$ result employs asymmetric b-tagging where only one b-tagging sample is used. In this sample one jet tagged 


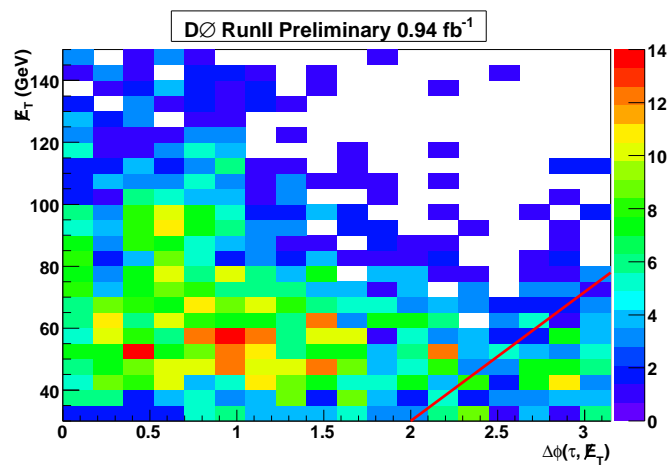

(a) signal

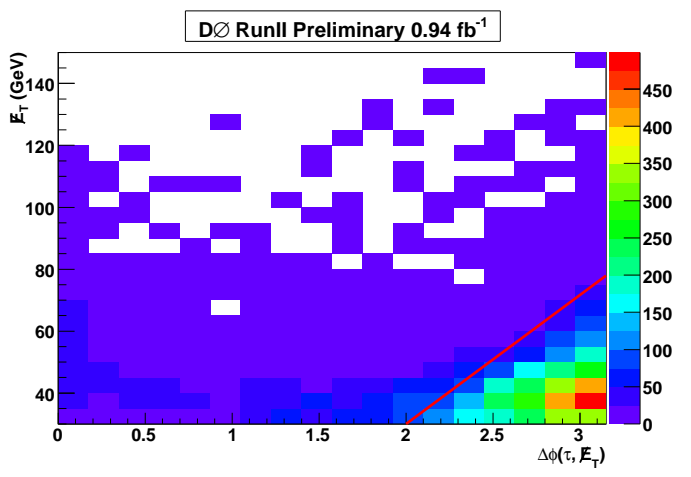

(b) multi-jet

Figure 7.4: $\Delta \phi\left(\tau, E_{\mathrm{T}}\right)$ vs $E_{\mathrm{T}}$ distributions for (a) signal and (b) multi-jet samples for the $1 \mathrm{fb}^{-1}$ result. The red line indicates where a cut is placed to remove a large amount of the multi-jet background. [50].

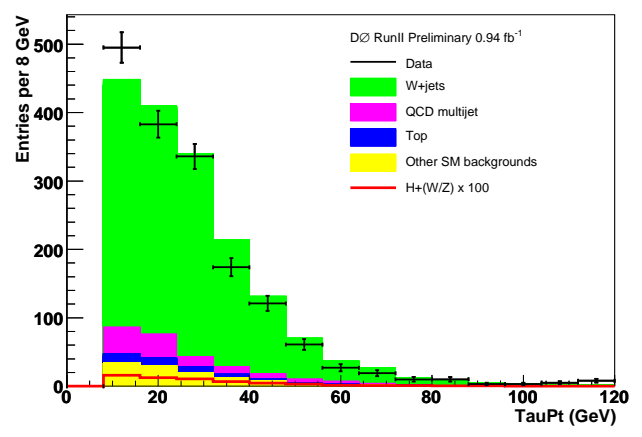

(a) $\tau$ transverse momentum

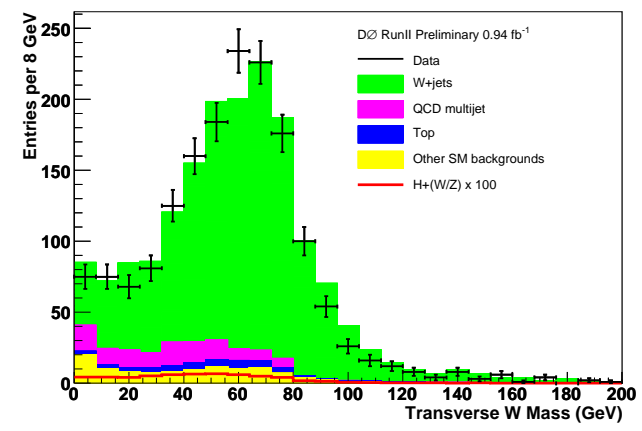

(b) transverse $\mathrm{W}$ mass

Figure 7.5: (a) $\tau$ transverse momentum and (b) transverse $\mathrm{W}$ mass distributions for the selected candidate events in the $1 \mathrm{fb}^{-1}$ analysis. Type 1 and $2 \tau$ candidate events have been added [50].

with the VeryTight operating point and one jet tagged with the L3 operating point are required. The di-jet mass distributions before and after this asymmetric btagging selection are shown in Figure 7.6. The final sample is comprised of mainly $W+$ heavy flavour jets and $t \bar{t}$ events.

The $1 \mathrm{fb}^{-1} W H \rightarrow \tau \nu b \bar{b}$ result was combined with the $1 \mathrm{fb}^{-1}(Z / W) H \rightarrow \tau \tau q \bar{q}$ search; this result was the first published SM Higgs search in tau final states [10]. The benefit of correlating systematics between the channels allowed the limits to improve more than if the channels were kept completely independent. The 


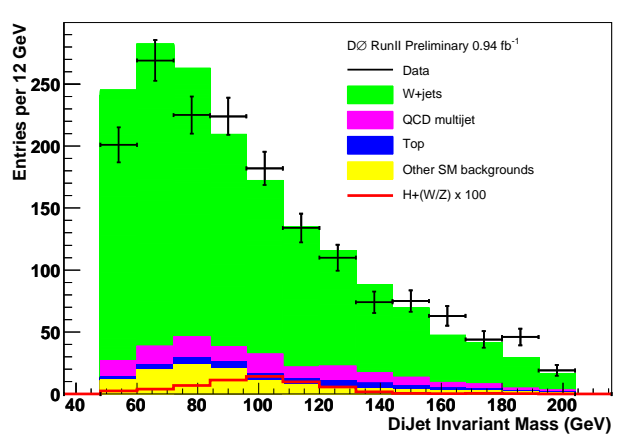

(a) Di-jet mass before b-tagging

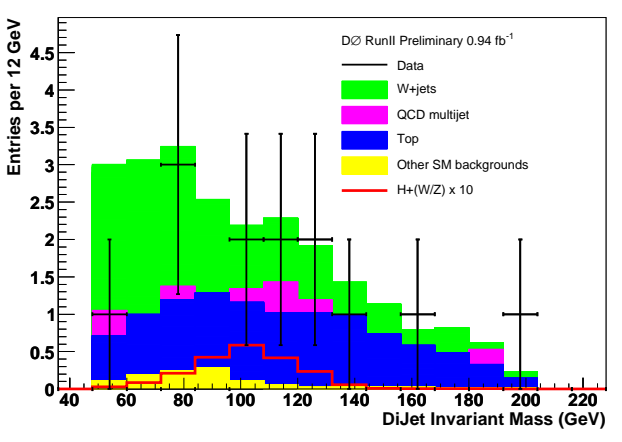

(b) Di-jet mass after b-tagging

Figure 7.6: Di-jet mass distributions (a) before b-tagging and (b) after b-tagging the sum of type 1 and 2 tau candidate events for the $1 \mathrm{fb}^{-1}$ result [50].

individual channels and combined limits are shown in Table 7.3 [10] across the range of Higgs masses tested.

\begin{tabular}{|c|cc|cc|cc|}
\hline & \multicolumn{2}{|c|}{$\tau \nu$ analysis } & \multicolumn{2}{|c|}{$\tau \tau$ analysis } & \multicolumn{2}{c|}{ Combined } \\
\hline$m_{H}(\mathrm{GeV})$ & exp. & obs. & exp. & obs. & exp. & obs. \\
\hline 105 & 33 & 27 & 39 & 36 & 24 & 20 \\
\hline 115 & 42 & 35 & 43 & 47 & 28 & 29 \\
\hline 125 & 62 & 60 & 60 & 65 & 40 & 44 \\
\hline 135 & 105 & 106 & 87 & 61 & 63 & 50 \\
\hline 145 & 226 & 211 & 158 & 95 & 120 & 82 \\
\hline
\end{tabular}

Table 7.3: Individual and combined limits for the ratio of excluded cross-sections over the SM cross-section for the Higgs in tau final states search channels.

\subsubsection{Improvements over the $1 \mathrm{fb}^{-1}$ Result}

In extending the $W H \rightarrow \tau \nu b \bar{b}$ search to the $4 \mathrm{fb}^{-1}$ data set a number of degrading effects had to be overcome. Due to the instantaneous luminosity increase between the RunIIa and RunIIb portion of the data, losses were noticed in tau-lepton identification efficiency $(\sim 5 \%)$ and trigger efficiency for the signal $(\sim 5-10 \%)$. However, the improvement in the limits moving from $1 \mathrm{fb}^{-1}$ to $4 \mathrm{fb}^{-1}$ is still approximately a factor of two. This is mainly due to the improvements in the 
analysis technique listed below:

- The use of a boosted decision tree allowed a looser selection and signal efficiency to be increased.

- The boosted decision tree utilises other variables, in addition to the di-jet mass to gain signal to background discrimination.

- The use of two b-tagging samples has increased the signal acceptance and allowed more statistics for the fitting of systematics uncertainties, allowing better constraints on the total systematic.

Although not directly related to an improvement in the analysis limits, the improved understanding of the multi-jet background by reweighting certain tau variables has yielded a better modelling of the background at the preselection stage of the analysis.

\subsubsection{Future Improvements to the $W H \rightarrow \tau \nu b \bar{b}$ Search}

For future iterations of the $W H \rightarrow \tau \nu b \bar{b}$ search improvements are most likely to be found in obtaining higher trigger efficiency for the signal and improved tau-lepton identification efficiency.

For the most recent data new tau + jets triggers are online benefiting from new L1 calorimeter tau trigger terms. These terms were studied by the author and are very similar to the L1 calorimeter jet terms with the addition of an isolation criteria on the calorimeter energy deposit. By requiring that the ratio of energy deposited in a $2 \times 2$ region of calorimeter towers $\left(E_{2 \times 2}\right)$ divided by the energy deposited in a $4 \times 4$ region $\left(E_{4 \times 4}\right)$ be greater than some threshold (nominally 0.6 ), more collimated jets are accepted. Most hadronic tau decays in the detector have a ratio $\frac{E_{2 \times 2}}{E_{4 \times 4}}>0.7$. By rejecting a large amount of the jet fakes, the rate at which 
data is written to storage is reduced. It is then possible to reduce the transverse momentum cut at the trigger level, optimising the rate again and increasing the signal acceptance.

Improvements in tau-lepton identification efficiency are more difficult. Studies are being performed to look at new variables which could be added to the tau neural network to further improve signal to background discrimination. Additionally, other tau signal samples are being trained to determine whether the topology of the tau event can offer more discrimination, but these studies are still a work in progress. 


\section{Chapter 8}

\section{Conclusions}

The first search for a Standard Model Higgs boson in the $\tau \nu b \bar{b}$ channel at the D $\varnothing$ experiment is presented. The analysis uses data corresponding to an integrated luminosity of $4 \mathrm{fb}^{-1}$, collected between 2002 and 2009. No significant excess is observed in the data and limits are set on the SM Higgs production cross-section. A cross-section 14.1 times the predicted SM cross-section for associated Higgs boson production is excluded at the $95 \%$ confidence level for a Higgs boson mass $m_{H}$ of $115 \mathrm{GeV}$.

Events are triggered using a suite of multi-jet triggers which require a certain number of jets in the event. Additionally, requirements are made on the angle between jets, missing transverse energy and other topological variables such as the scalar and vector sum of jet energies. The $\tau \nu b \bar{b}$ final state is selected in data by requiring the missing transverse energy in the event to be greater than $15 \mathrm{GeV}$ to identify the neutrino. A reconstructed tau candidate with $p_{T}>15,10 \mathrm{GeV}$ dependent on tau type is required. Taus are identified using a neural network trained to increase the hadronic tau decay purity against jet fakes. A requirement of $\tau_{n n_{h}}>0.9$ is placed on this output. The events must have at least two jets with $p_{T}>15 \mathrm{GeV}$ and be well separated from the tau candidate. Sensitivity to 
the $W H \rightarrow e \nu b \bar{b}$ signal is gained from electrons that are reconstructed as taus and selected in this analysis. To avoid double counting of events across analyses, a veto is imposed on events selected in the $W H \rightarrow(e / \mu) \nu b \bar{b}$ analysis.

The dominant background at this stage of the analysis arises from multi-jet events. This contribution is estimated from a sideband region in data where the tau candidate has a neural network output between 0.3 and 0.7 . This region is dominated by jets faking taus. Multi-jet events are normalised in a control region that has no overlap with the final analysis sample. A kinematic reweighting is applied to the events to account for mis-modelling of tau kinematic variables in the control region.

The most discriminating variable between signal events and the multi-jet background is the missing transverse energy significance $\left(E_{\mathrm{T}_{\mathrm{SIG}}}\right)$. The angle $\Delta \phi\left(\not P_{\mathrm{T}}, E_{\mathrm{T}}\right)$ offers additional discrimination between signal events and the multijet background. The analysis requires $\mathbb{E}_{\mathrm{T}_{\mathrm{SIG}}}>4.5$ and $\Delta \phi\left(\not_{\mathrm{T}}, \mathbb{E}_{\mathrm{T}}\right)<2$, which removes around $80 \%$ of the multi-jet contribution.

After this selection, the analysis sample is dominated by $W$ events with two associated jets, which mainly originate from light quarks. These events are modelled by MC simulation and scaled to the appropriate theoretical cross-sections. A MC control sample is defined by requiring $\mathbb{F}_{\mathrm{T}}>80 \mathrm{GeV}$ to study the agreement between the simulation and data; the description is found to be good. To increase the sensitivity to the signal, b-tagging requirements are imposed on the jets in the events. One sample requires that the event contains one tight operating point b-tagged jet, the other sample requires that the event contains two loose operating point b-tagged jets.

To further increase the signal to background ratio in the final analysis sample a boosted decision tree (BDT) is trained. This allows other variables to contribute to the signal - background separation rather than using the di-jet mass 
alone. $W H \rightarrow \tau \nu b \bar{b}$ events are used as signal, and are trained against all simulated backgrounds (not multi-jet events). Eight separate samples are used in the BDT representing each of the tau type, jet multiplicity and b-tagging configurations. Separating the channels this way improves the sensitivity, especially in the channels containing two associated jets, as most of the $t \bar{t}$ events fall in the three jet sample.

Uncertainties are classified to be Flat or Shape systematics depending on their effect on the BDT output. The degrading effect of systematic uncertainties is reduced by a minimisation fit procedure. However, even with the fitting procedure, systematic uncertainties still degrade the expected limit by around $30 \%$.

The analysis presented in this thesis is an update to the previous version of the analysis using $1 \mathrm{fb}^{-1}$, also performed by the author, which was the first analysis of the $W H \rightarrow \tau \nu b \bar{b}$ channel [50]. Additionally, a combination was performed by the author with the $(Z / W) H \rightarrow \tau \tau q \bar{q}$ analysis, which was the first published SM Higgs search in a tau decay mode [10]. The improvement in the expected limit presented in this thesis over the $1 \mathrm{fb}^{-1}$ result is a factor of 1.88. A factor of two would be predicted by luminosity gains alone. The increase in instantaneous luminosity has reduced the efficiency of tau-identifaction and reduced the trigger efficiency. Improvements in the analysis technique, such as the loosening of cuts to increase signal acceptance and the use of a boosted decision tree to improve signal - background separation, regained some of the sensitivity from these losses.

The $W H \rightarrow \tau \nu b \bar{b}$ channel has added sensitivity to the D $\varnothing$ and Tevatron SM Higgs searches. The additional sensitivity gained from this channel is equivalent to around a $10 \%$ integrated luminosity increase in the $W H \rightarrow(e / \mu) \nu b \bar{b}$ analyses. Only with the addition of channels like $W H \rightarrow \tau \nu b \bar{b}$ will sensitivity to a low mass Higgs at the Tevatron be possible before the end of running and such analyses will help keep the Tevatron at the frontier of Higgs searches. 


\section{Appendix A}

\section{Kinematic Distributions}

This section shows a larger range of kinematic distributions that were not included in the main text. Many of these variables are used in the training of the boosted decision tree (see Section 6.1.2). A short description of the variables shown here is given below:

- Di-jet mass is the invariant mass using the two leading (highest $p_{T}$ ) jets in the event.

- Jet multiplicity is the number of jets in the event.

- $p_{T}($ jet 1$)$ is the transverse momentum of the leading jet in the event.

- $p_{T}($ jet 2$)$ is the transverse momentum of the next-to-leading jet in the event.

- $\eta_{d}($ jet 1$)$ is the detector $\eta$ of the leading jet in the event.

- $\eta_{d}($ jet 2$)$ is the detector $\eta$ of the next-to-leading jet in the event.

- $\Delta \phi($ jet 1, jet 2$)$ is the angle $\phi$ between the two leading jets in the event.

- $\Delta R($ jet 1, jet 2$)$ is the distance $R$ between the two leading jets in the event. 
- $\Delta R(\tau, j e t s)$ is the distance $R$ between the tau candidate and any jets in the event.

- $p_{T}(\tau)$ is the transverse momentum of the tau candidate.

- $\eta_{d}(\tau)$ is the detector $\eta$ of the tau candidate.

- $\mathbb{E}_{\mathrm{T}_{\mathrm{SIG}}}$ is the missing transverse energy significance of the event.

- $m_{T}(W)$ is the transverse $\mathrm{W}$ mass constructed from the $\tau$ and $\mathbb{E}_{\mathrm{T}}$ in the event.

- $\mathbb{E}_{\mathrm{T}}$ is the missing transverse energy in the event.

- $\Delta \phi\left(P_{\mathrm{T}}, \mathbb{F}_{\mathrm{T}}\right)$ is the angle $\phi$ between the missing transverse energy, $\mathbb{F}_{\mathrm{T}}$ and the missing transverse energy from tracks alone, $\not_{\mathrm{T}}$. 


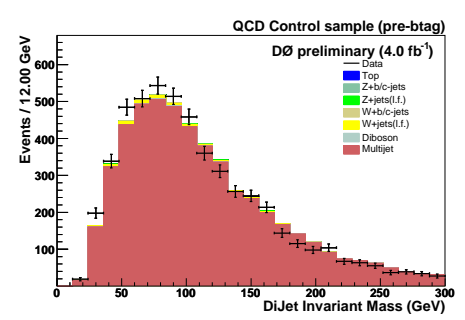

(a) Di-jet Mass

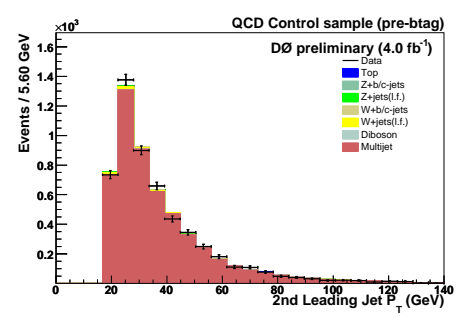

(d) $p_{T}($ jet 2$)$

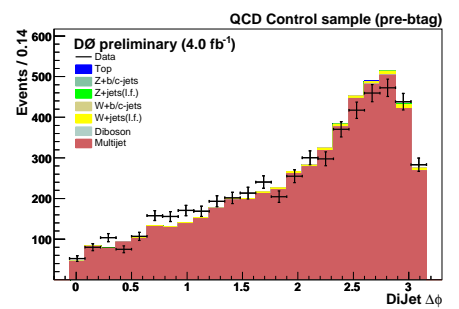

(g) $\Delta \phi($ jet1,jet2)

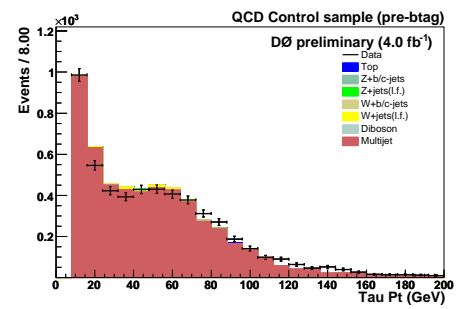

(j) $p_{T}(\tau)$

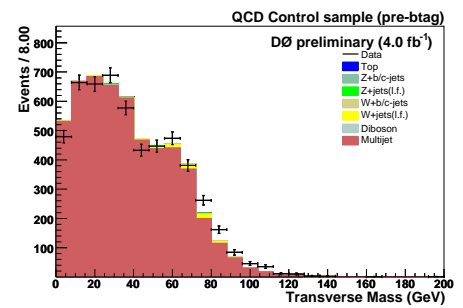

(m) $m_{T}(W)$

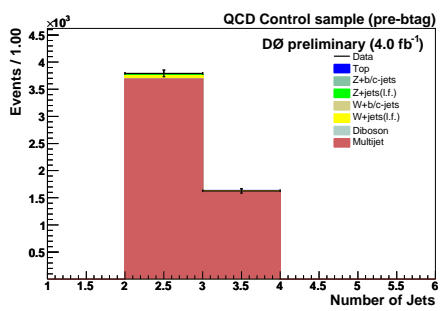

(b) Jet Multiplicity

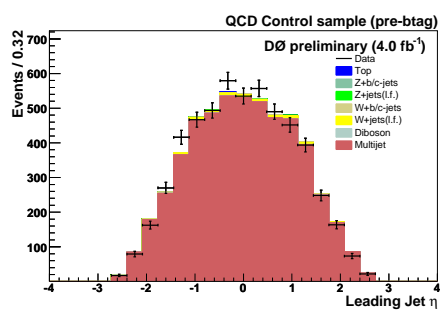

(e) $\eta_{d}(j e t 1)$

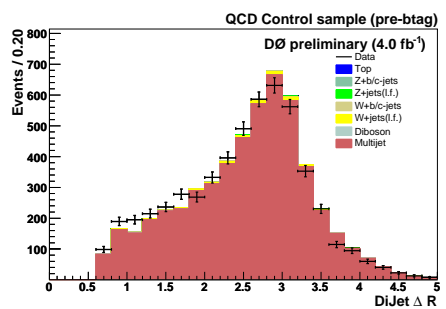

(h) $\Delta \mathrm{R}($ jet1,jet2)

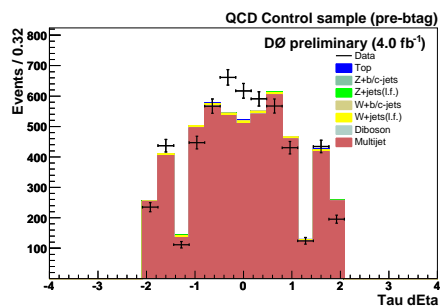

(k) $\eta_{d}(\tau)$

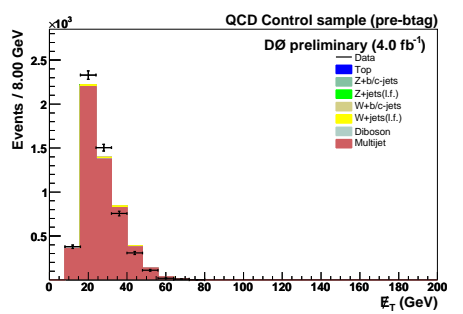

(n) $\not_{\mathrm{T}}$

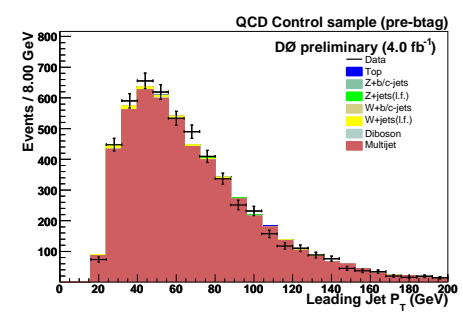

(c) $p_{T}(j e t 1)$

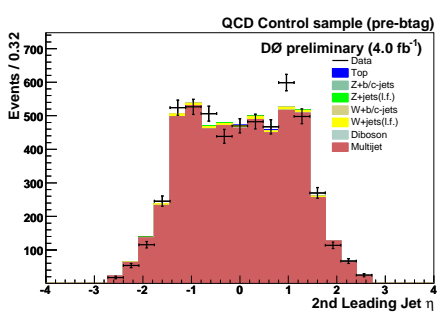

(f) $\eta_{d}(j e t 2)$

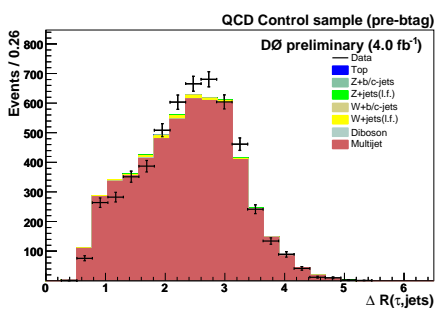

(i) $\Delta \mathrm{R}($ tau,jets $)$

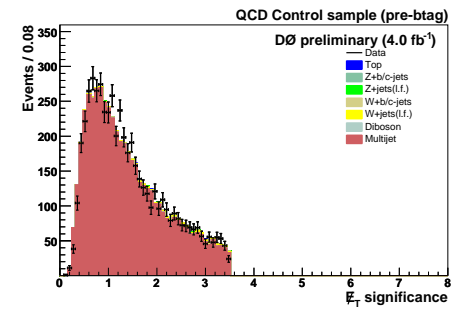

(l) $\not_{\mathrm{T}_{\mathrm{SIG}}}$

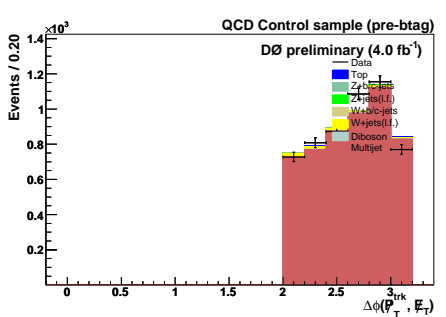

(o) $\Delta \phi\left(\not P_{\mathrm{T}}, \not_{\mathrm{T}}\right)$

Figure A.1: Data - Background comparisons in the multi-jet control sample with a $p_{T}(\tau)$ and $\eta_{d}(\tau)$ dependent normalisation factor for type 2 tau candidate events. 


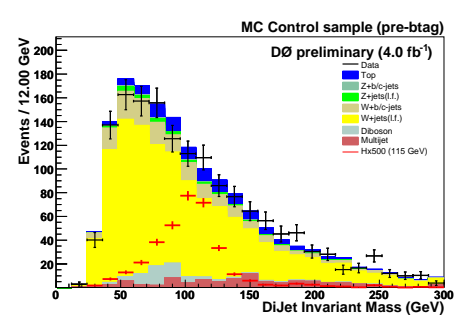

(a) Di-jet Mass

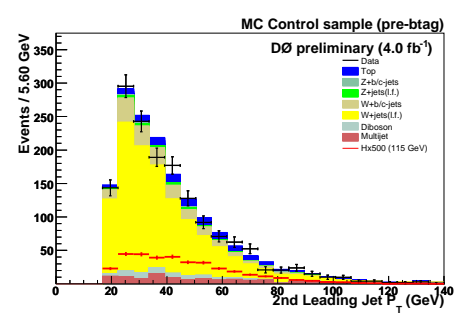

(d) $p_{T}(j e t 2)$

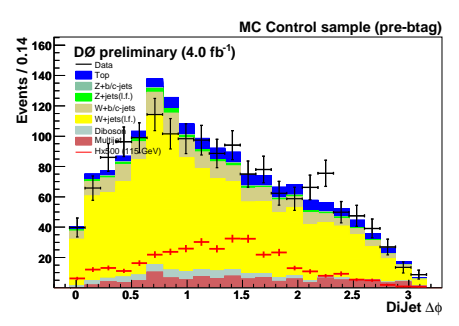

(g) $\Delta \phi($ jet1,jet2)

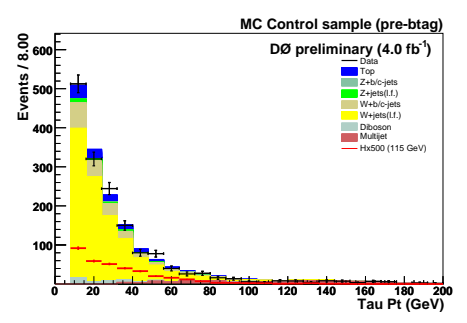

(j) $p_{T}(\tau)$

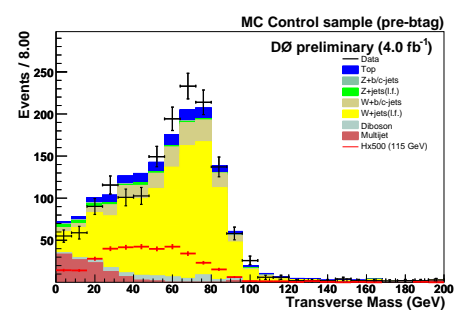

(m) $m_{T}(W)$

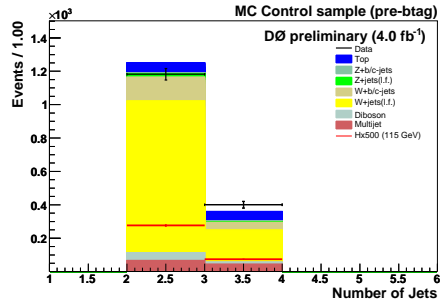

(b) Jet Multiplicity

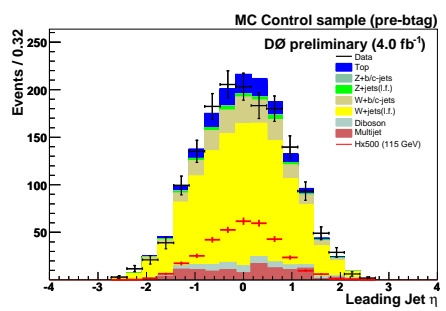

(e) $\eta_{d}(j e t 1)$

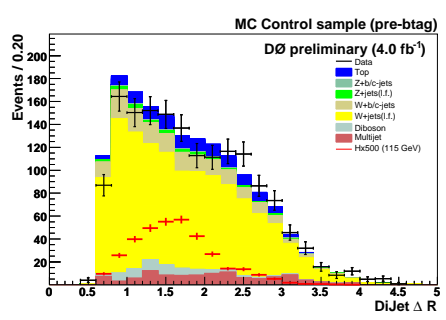

(h) $\Delta R($ jet1,jet2)

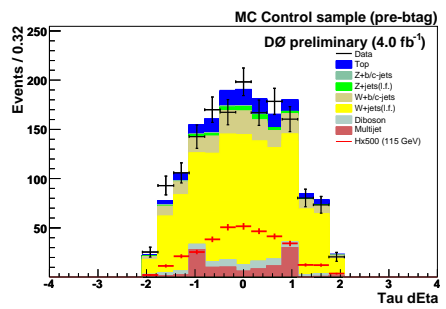

(k) $\eta_{d}(\tau)$

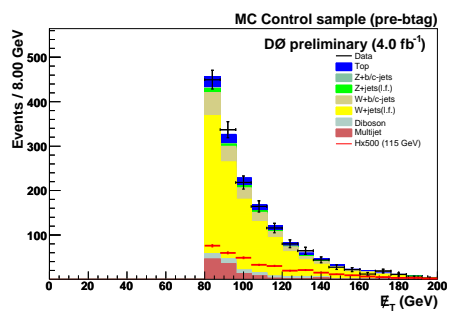

(n) $\mathbb{E}_{\mathrm{T}}$

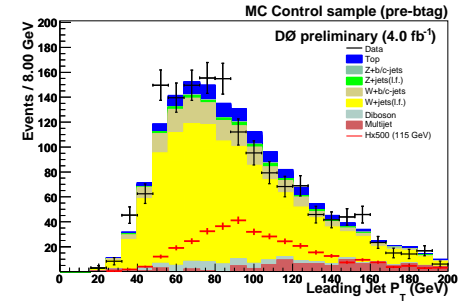

(c) $p_{T}($ jet 1$)$

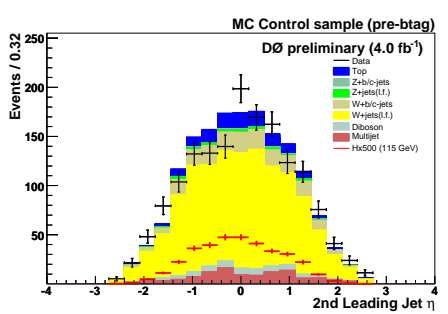

(f) $\eta_{d}(j e t 2)$

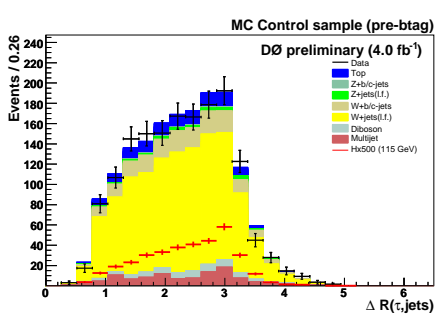

(i) $\Delta \mathrm{R}($ tau,jets $)$

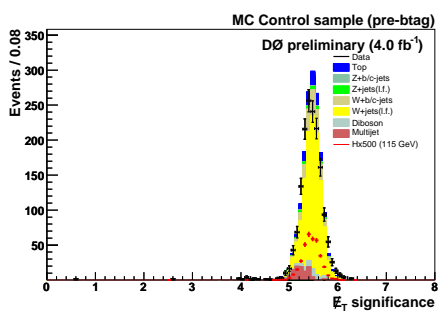

(l) $\#_{T_{\text {SIG }}}$

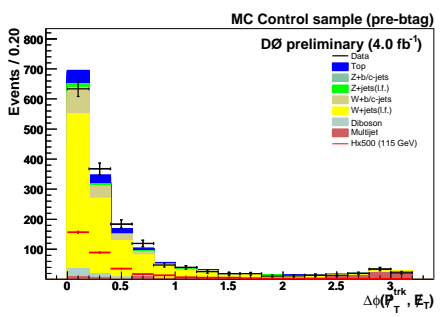

(o) $\Delta \phi\left(\not P_{\mathrm{T}}, \not_{\mathrm{T}}\right)$

Figure A.2: Data - Background comparisons in the MC control sample for type 2 tau candidate events. 


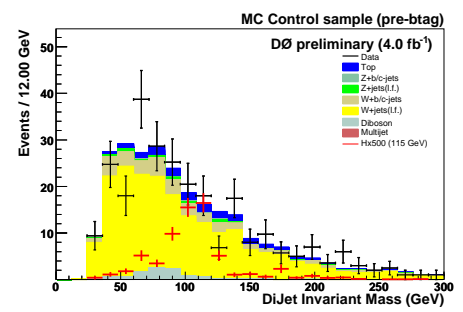

(a) Di-jet Mass

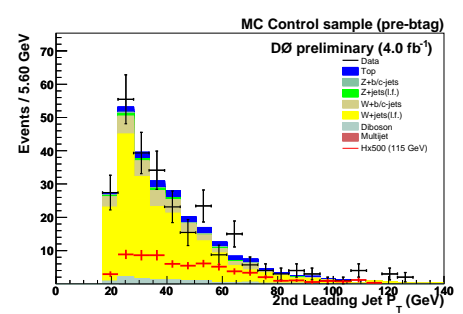

(d) $p_{T}($ jet 2$)$

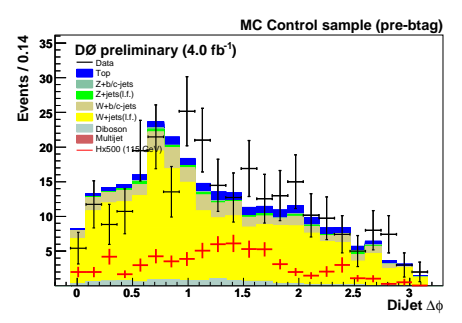

(g) $\Delta \phi($ jet1,jet2)

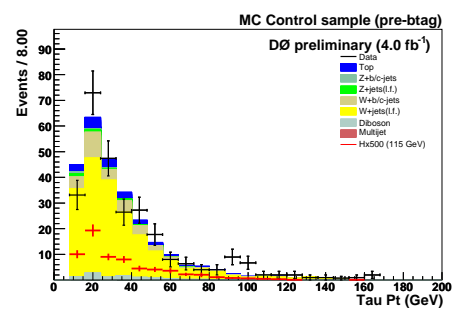

(j) $p_{T}(\tau)$

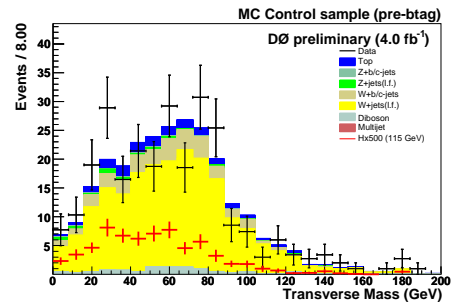

(m) $m_{T}(W)$

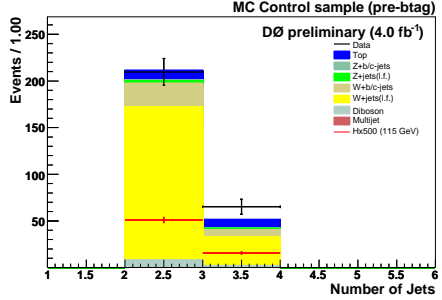

(b) Jet Multiplicity

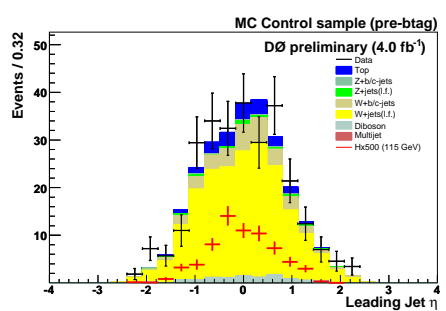

(e) $\eta_{d}($ jet 1$)$

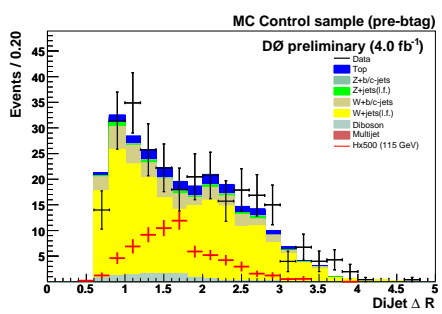

(h) $\Delta \mathrm{R}($ jet1,jet2)

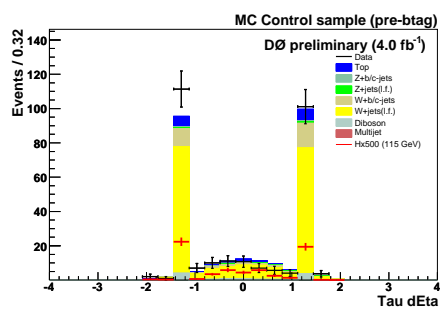

(k) $\eta_{d}(\tau)$

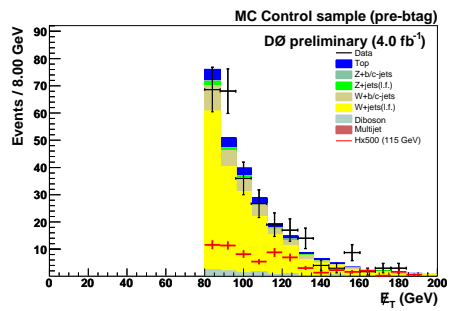

(n) $E_{\mathrm{T}}$

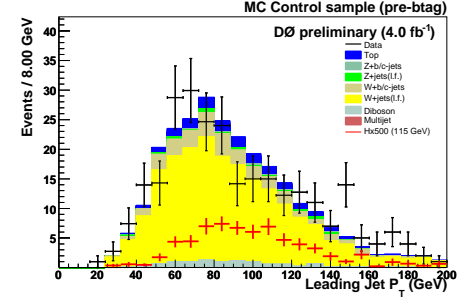

(c) $p_{T}($ jet 1$)$

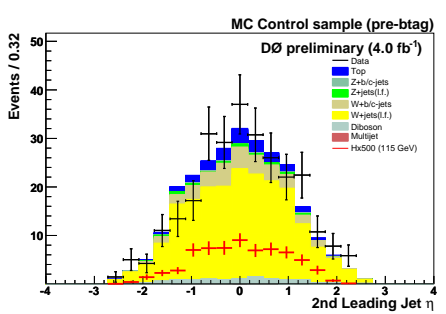

(f) $\eta_{d}($ jet 2$)$

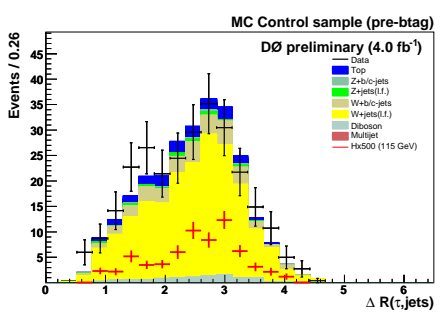

(i) $\Delta \mathrm{R}$ (tau,jets)

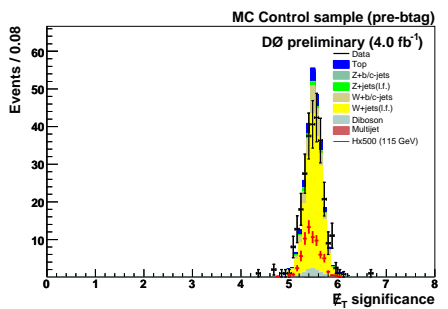

(l) $\not_{T_{\text {SIG }}}$

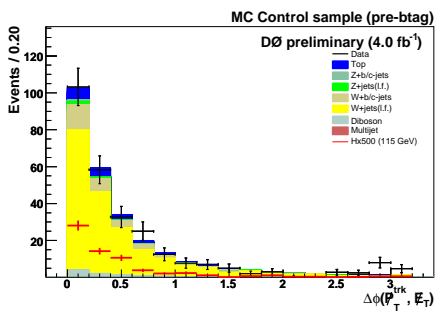

(o) $\Delta \phi\left(\not P_{\mathrm{T}}, E_{\mathrm{T}}\right)$

Figure A.3: Data - Background comparisons in the MC control sample for type 1 tau candidate events. 


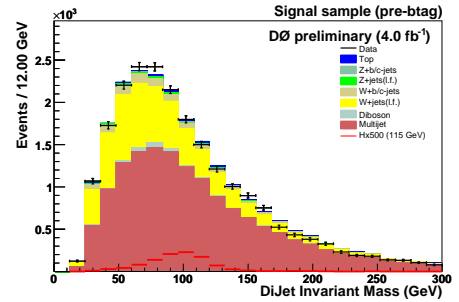

(a) Di-jet Mass

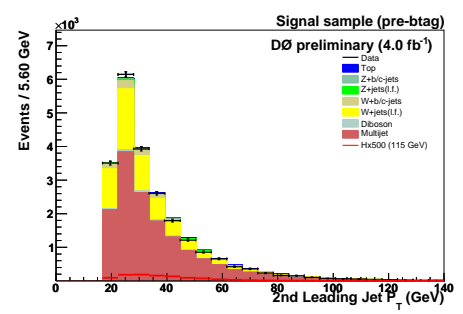

(d) $p_{T}($ jet 2$)$

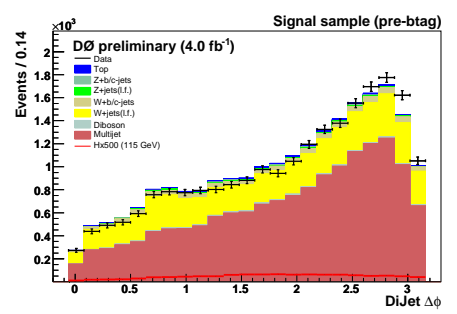

(g) $\Delta \phi($ jet 1, jet2 $)$

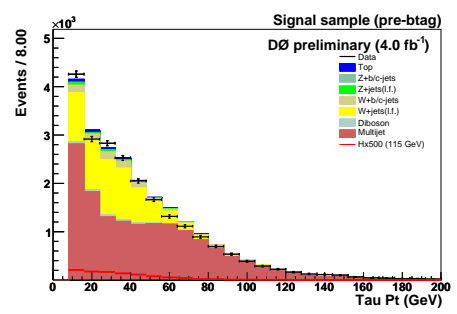

(j) $p_{T}(\tau)$

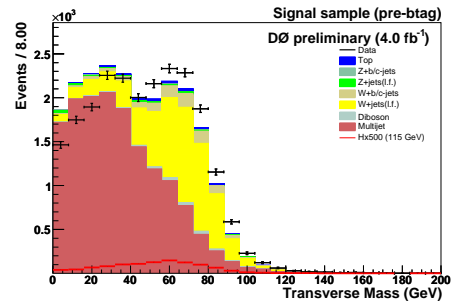

(m) $m_{T}(W)$

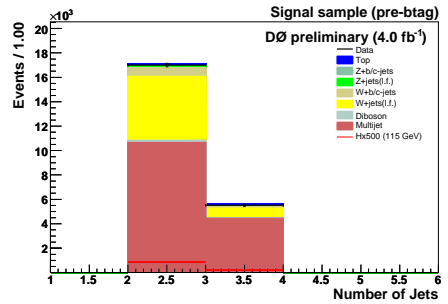

(b) Jet Multiplicity

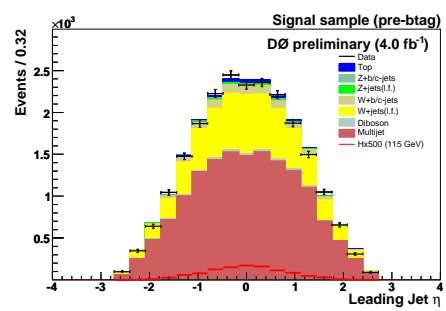

(e) $\eta_{d}($ jet 1$)$

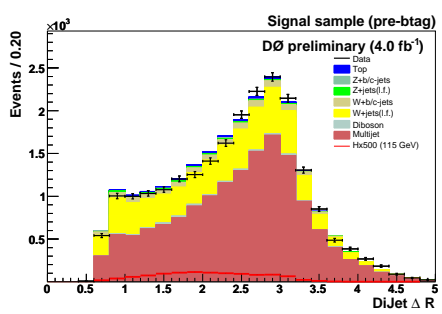

(h) $\Delta \mathrm{R}($ jet1,jet2)

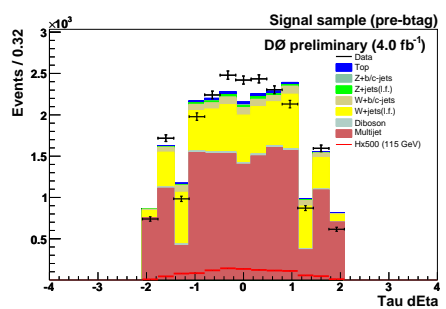

(k) $\eta_{d}(\tau)$

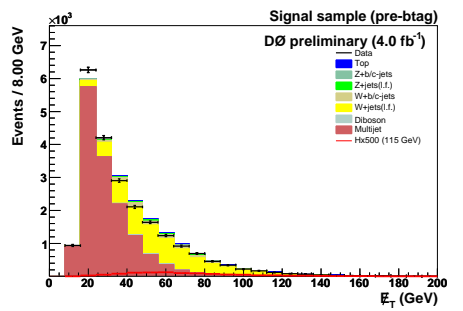

(n) $E_{\mathrm{T}}$

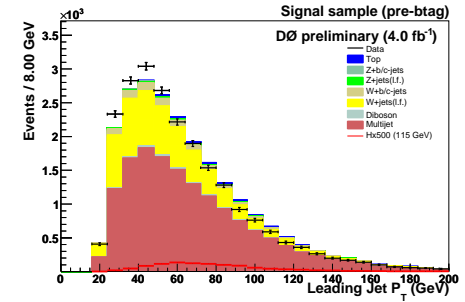

(c) $p_{T}($ jet 1$)$

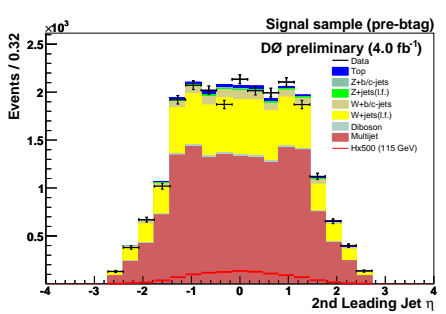

(f) $\eta_{d}($ jet 2$)$

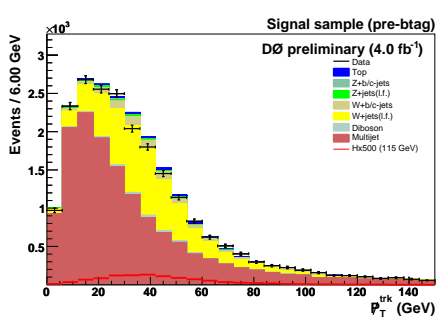

(i) $\not P_{\mathrm{T}}$

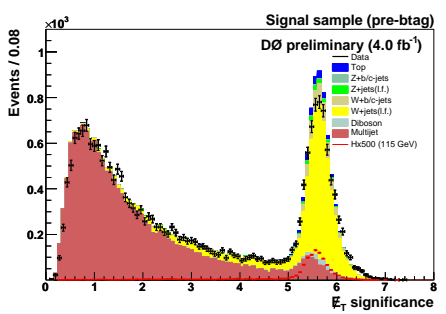

(l) $\mathscr{H}_{T_{\text {SIG }}}$

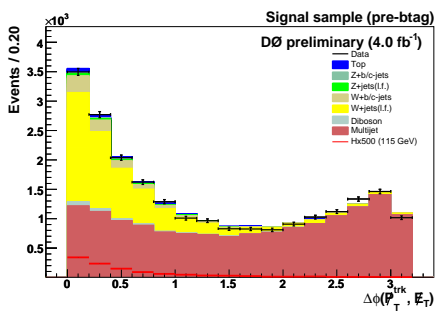

(o) $\Delta \phi\left(\not P_{\mathrm{T}}, E_{\mathrm{T}}\right)$

Figure A.4: Data - Background comparisons in the analysis sample after preselection for type 2 tau candidate events. 


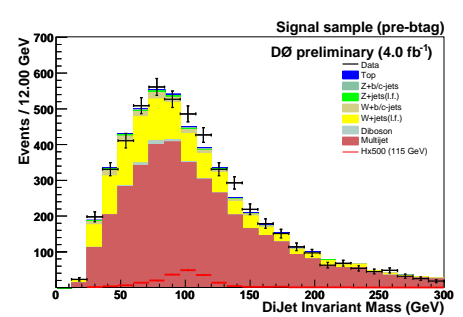

(a) Di-jet Mass

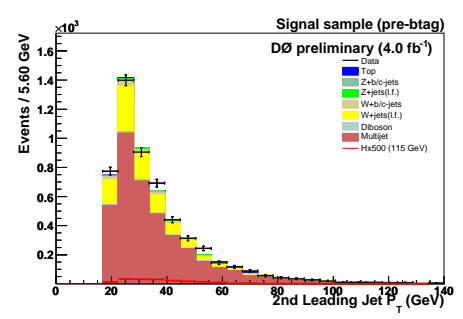

(d) $p_{T}(j e t 2)$

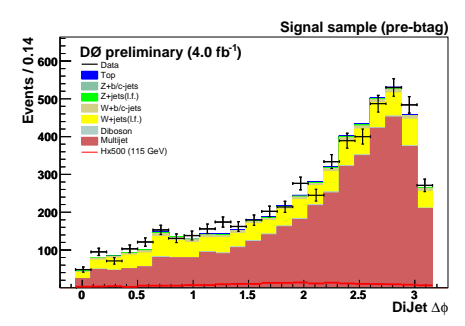

(g) $\Delta \phi($ jet1,jet2)

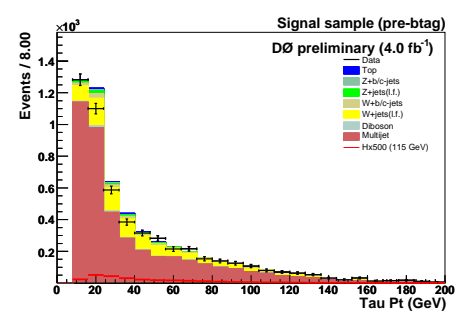

(j) $p_{T}(\tau)$

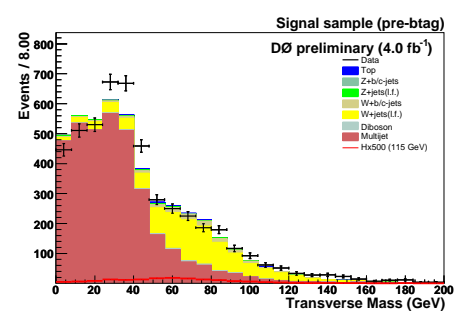

(m) $m_{T}(W)$

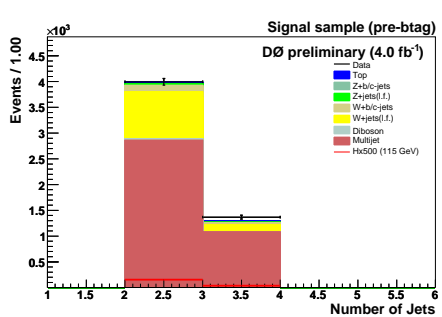

(b) Jet Multiplicity

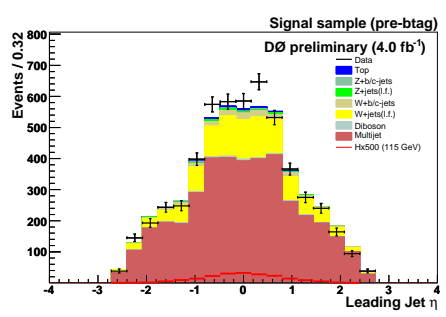

(e) $\eta_{d}(j e t 1)$

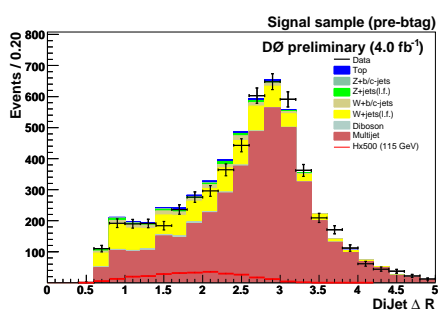

(h) $\Delta \mathrm{R}($ jet1,jet2)

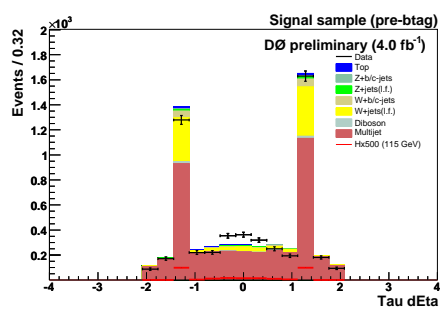

(k) $\eta_{d}(\tau)$

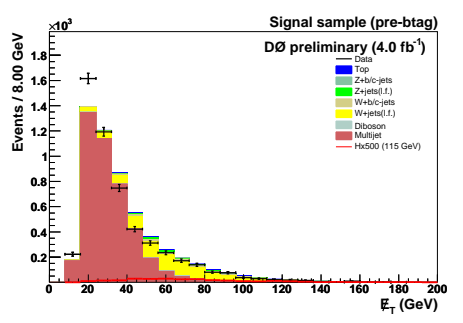

(n) $\not_{\mathrm{T}}$

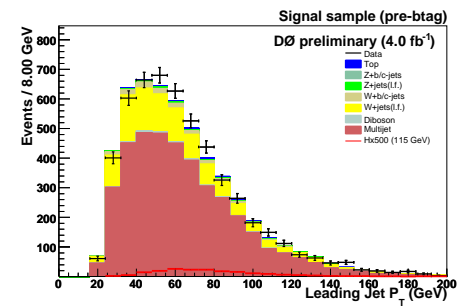

(c) $p_{T}(j e t 1)$

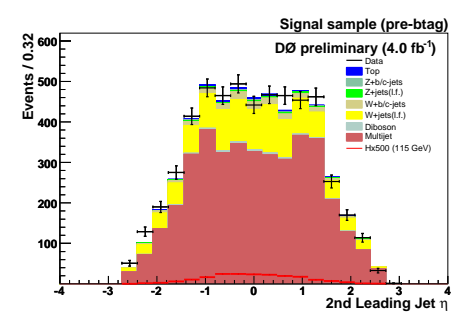

(f) $\eta_{d}(j e t 2)$

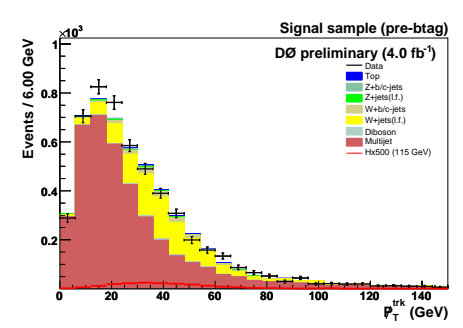

(i) $\not P_{\mathrm{T}}$

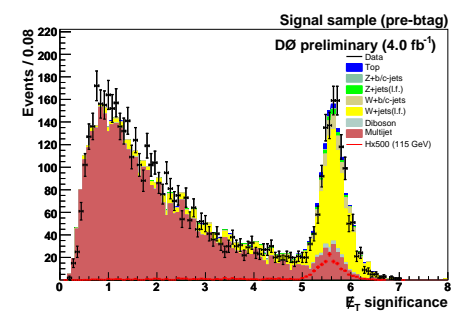

(l) $\#_{T_{\text {SIG }}}$

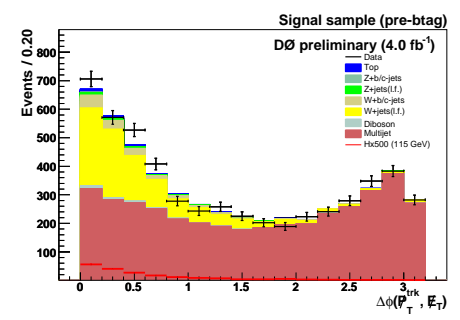

(o) $\Delta \phi\left(\not P_{\mathrm{T}}, \not_{\mathrm{T}}\right)$

Figure A.5: Data - Background comparisons in the analysis sample after preselection for type 1 tau candidate events. 


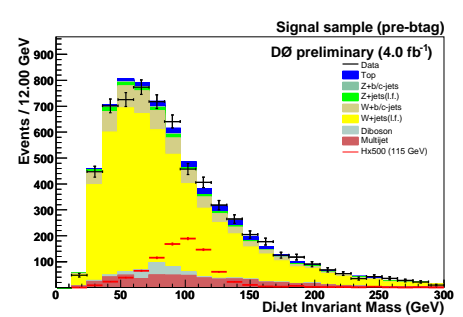

(a) Di-jet Mass

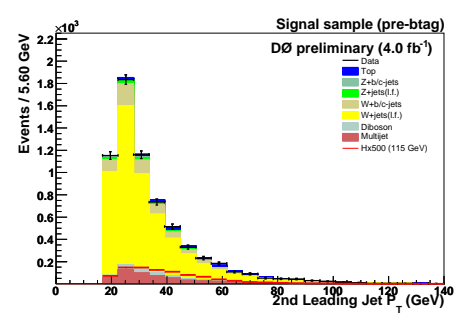

(d) $p_{T}($ jet 2$)$

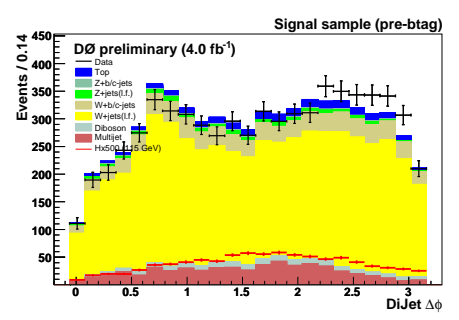

(g) $\Delta \phi($ jet1,jet2)

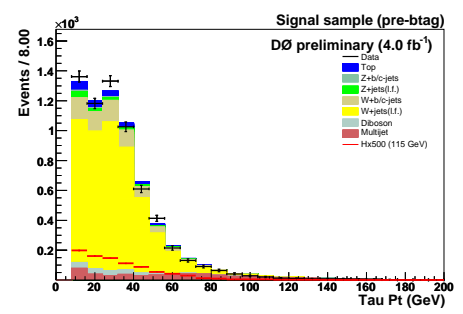

(j) $p_{T}(\tau)$

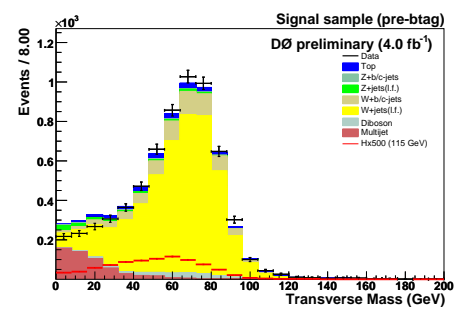

(m) $m_{T}(W)$

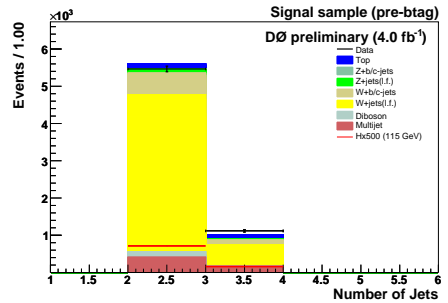

(b) Jet Multiplicity

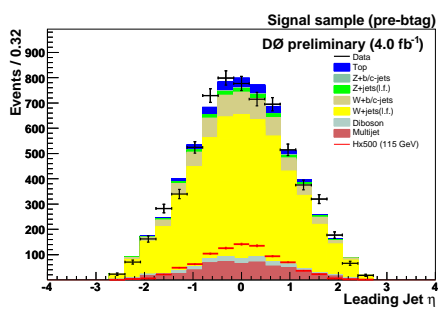

(e) $\eta_{d}($ jet 1$)$

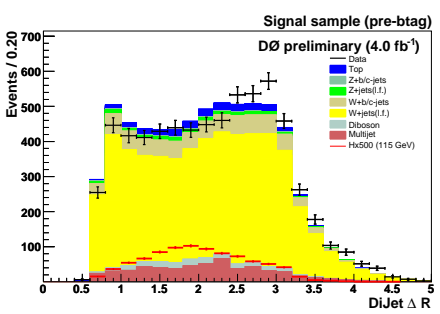

(h) $\Delta \mathrm{R}($ jet1,jet2)

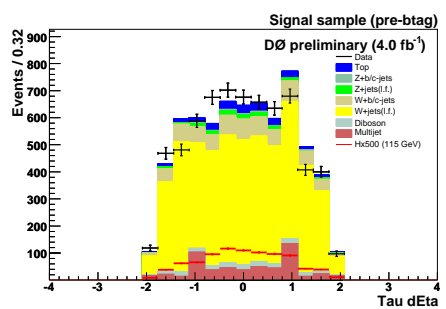

(k) $\eta_{d}(\tau)$

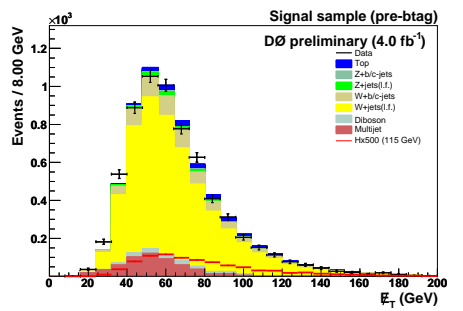

(n) $E_{\mathrm{T}}$

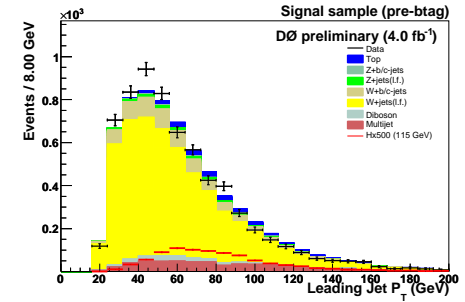

(c) $p_{T}($ jet 1$)$

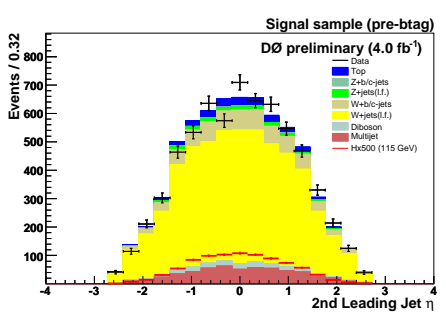

(f) $\eta_{d}($ jet 2$)$

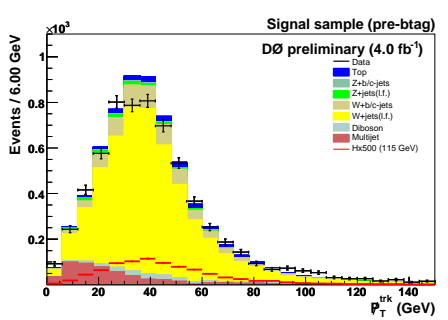

(i) $\not P_{\mathrm{T}}$

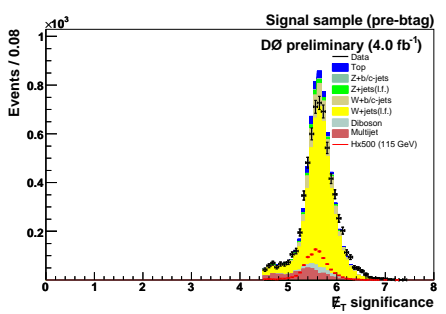

(l) $\mathscr{E}_{T_{\text {SIG }}}$

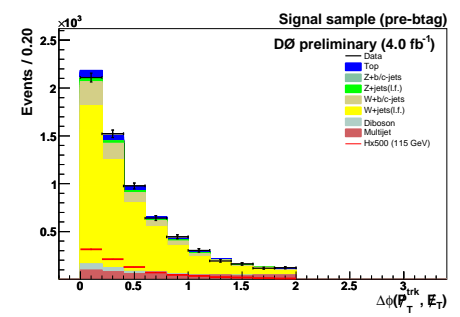

(o) $\Delta \phi\left(\not P_{\mathrm{T}}, E_{\mathrm{T}}\right)$

Figure A.6: Data - Background comparisons in the analysis sample after selection for type 2 tau candidate events. 


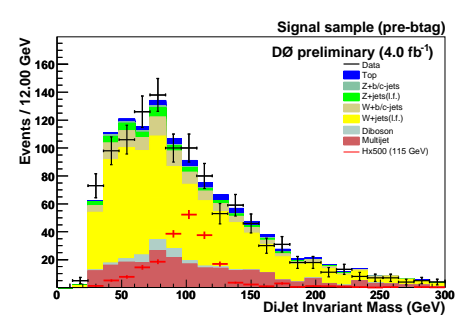

(a) Di-jet Mass

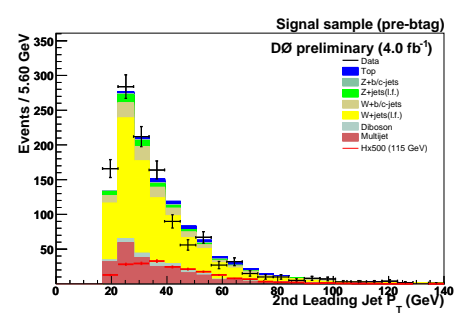

(d) $p_{T}($ jet 2$)$

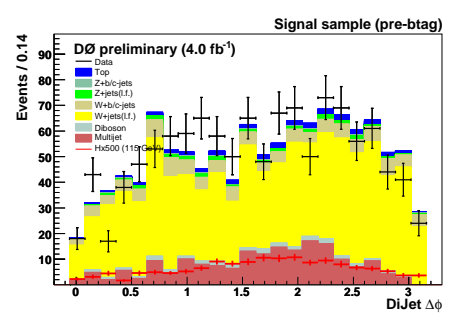

(g) $\Delta \phi($ jet1,jet2)

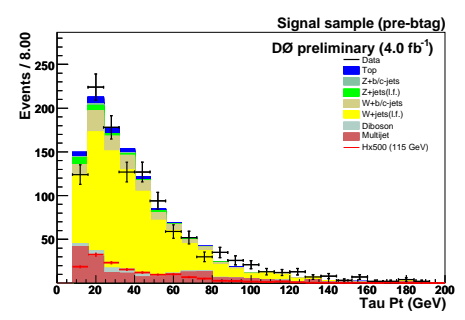

(j) $p_{T}(\tau)$

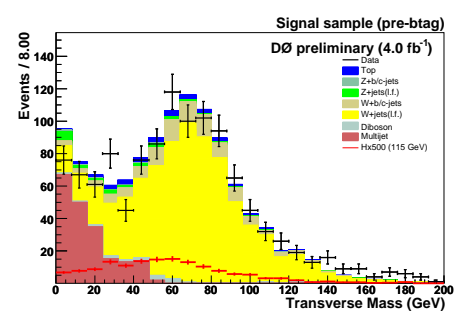

(m) $m_{T}(W)$

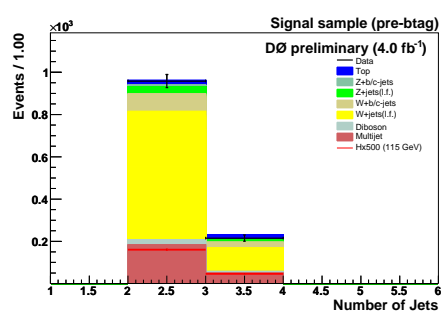

(b) Jet Multiplicity

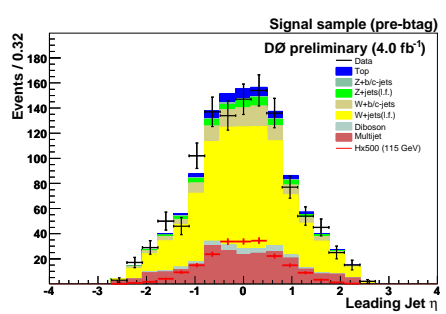

(e) $\eta_{d}($ jet 1$)$

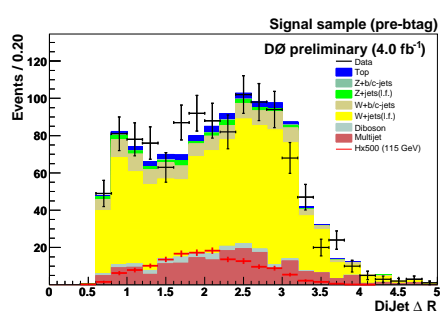

(h) $\Delta \mathrm{R}($ jet1,jet2)

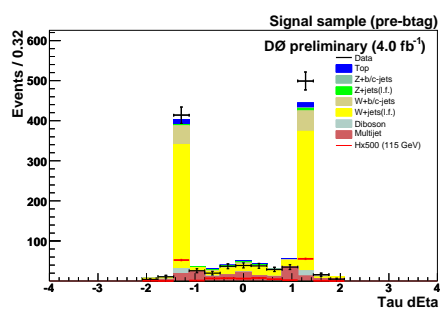

(k) $\eta_{d}(\tau)$

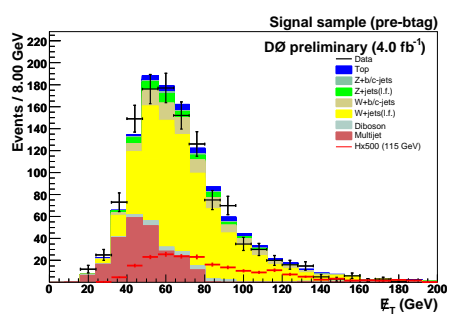

(n) $\ddot{E}_{\mathrm{T}}$

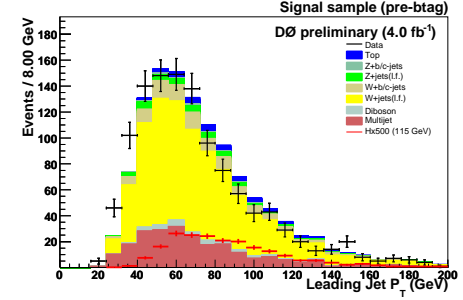

(c) $p_{T}($ jet 1$)$

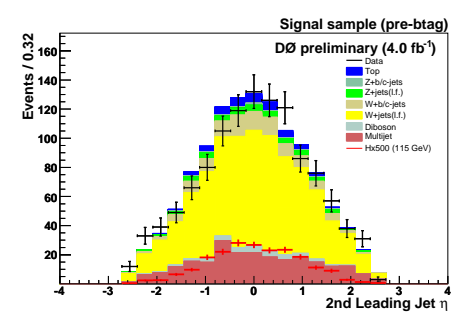

(f) $\eta_{d}($ jet2)

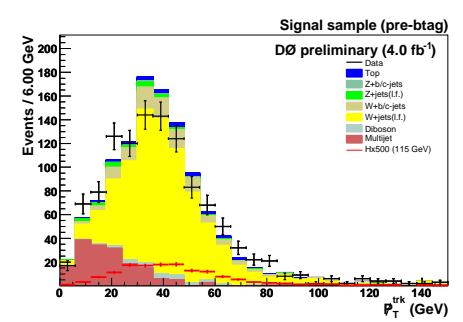

(i) $\not P_{\mathrm{T}}$

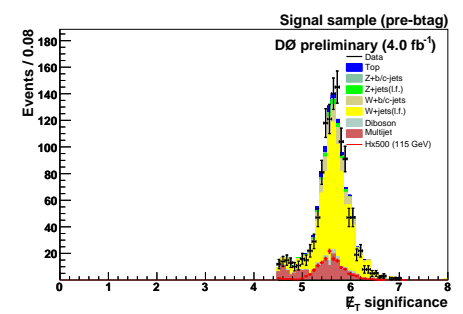

(l) $\mathscr{E}_{T_{\text {SIG }}}$

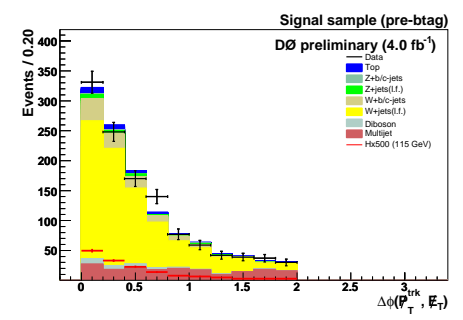

(о) $\Delta \phi\left(P_{\mathrm{T}}, E_{\mathrm{T}}\right)$

Figure A.7: Data - Background comparisons in the analysis sample after selection for type 1 tau candidate events. 


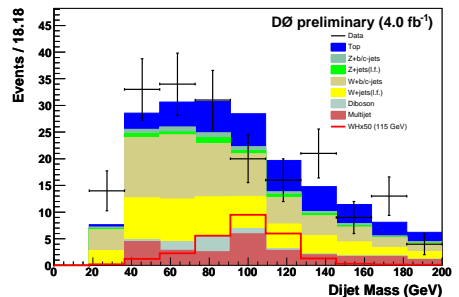

(a) Di-jet Mass

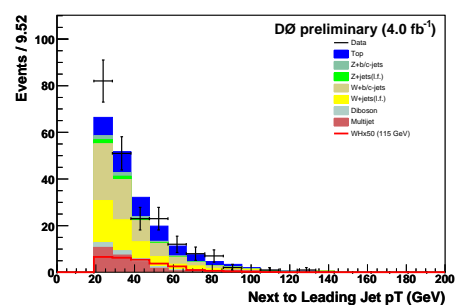

(d) $p_{T}($ jet 2$)$

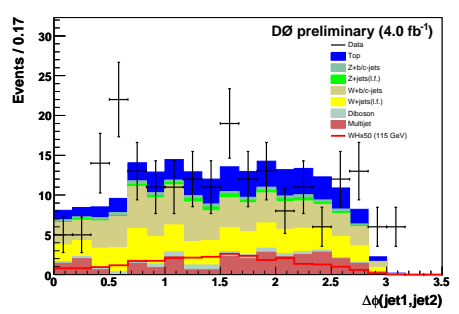

(g) $\Delta \phi($ jet1,jet2)

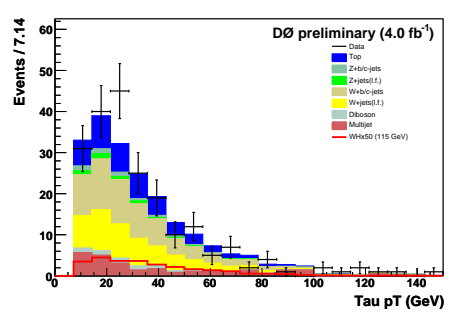

(j) $p_{T}(\tau)$

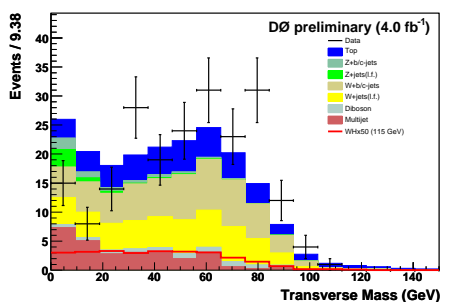

(m) $m_{T}(W)$

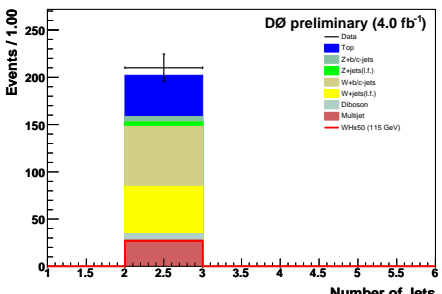

(b) Jet Multiplicity

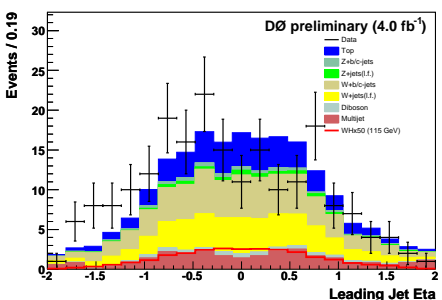

(e) $\eta_{d}($ jet 1$)$

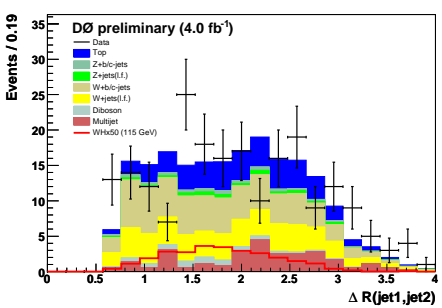

(h) $\Delta \mathrm{R}($ jet1,jet2)

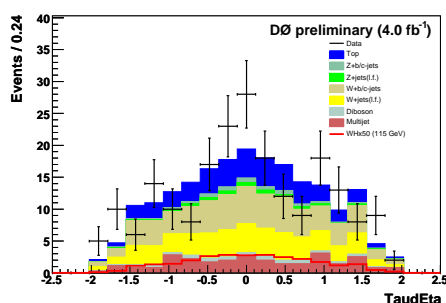

(k) $\eta_{d}(\tau)$

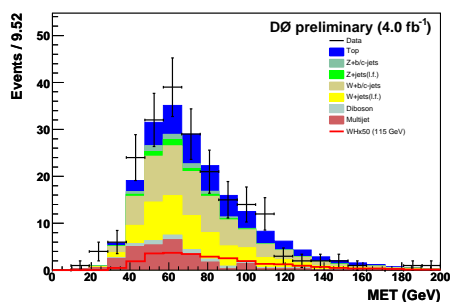

(n) $E_{\mathrm{T}}$

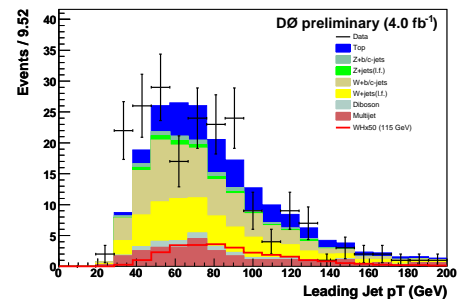

(c) $p_{T}(j e t 1)$

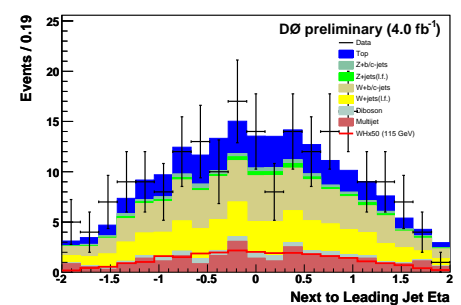

(f) $\eta_{d}(j e t 2)$

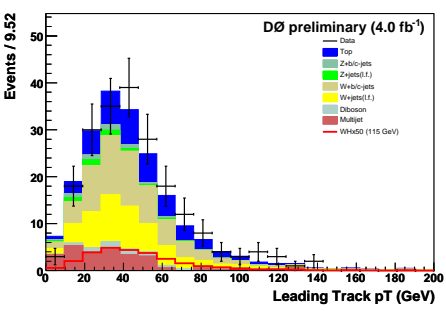

(i) $p_{T}(\operatorname{trk} 1)$

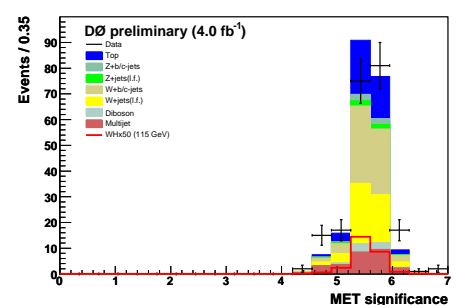

(1) $\ddot{E}_{\mathrm{T}_{\mathrm{SIC}}}$

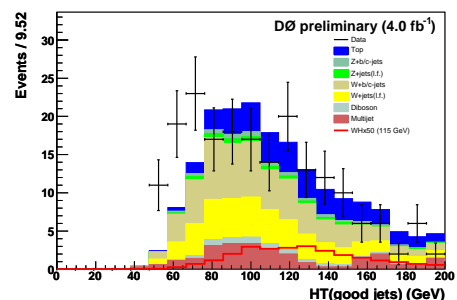

(o) $H_{T}$ (good jets)

Figure A.8: Data - Background comparisons in the analysis sample after one tight (VeryTight) b-tag selection for type 2 tau candidate events with 2 jets. 


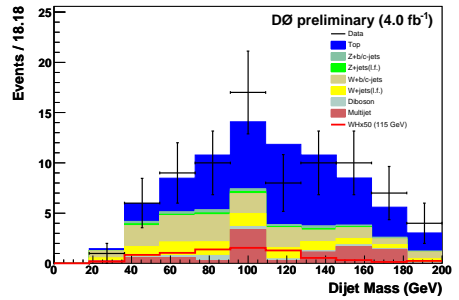

(a) Di-jet Mass

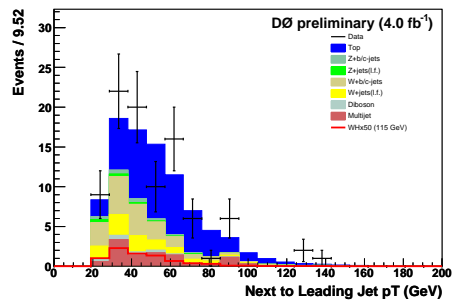

(d) $p_{T}(j e t 2)$

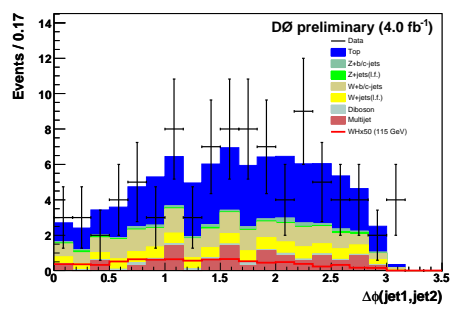

(g) $\Delta \phi($ jet1,jet2)

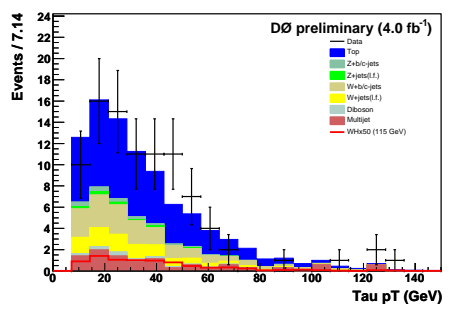

(j) $p_{T}(\tau)$

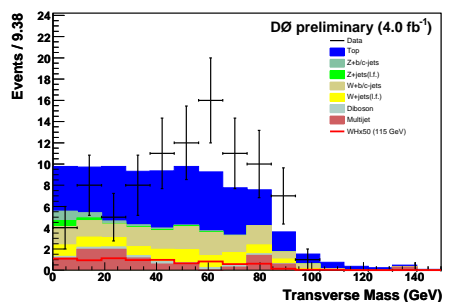

(m) $m_{T}(W)$

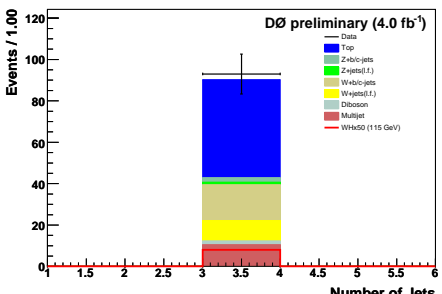

(b) Jet Multiplicity

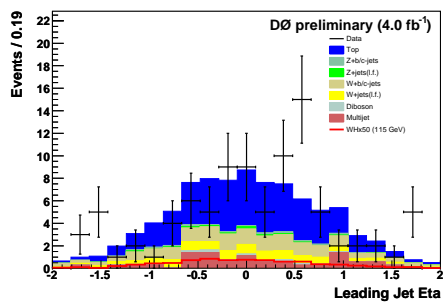

(e) $\eta_{d}(j e t 1)$

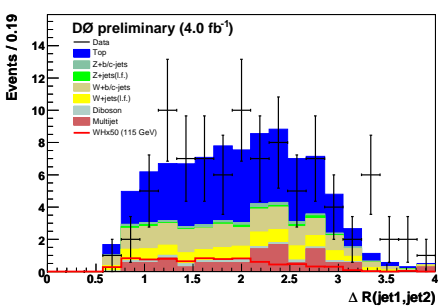

(h) $\Delta \mathrm{R}($ jet1,jet2)

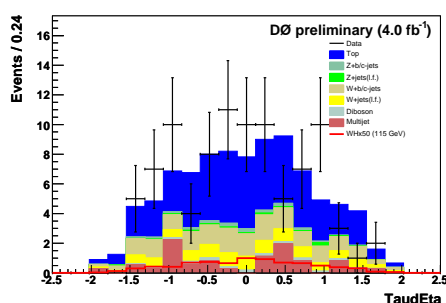

(k) $\eta_{d}(\tau)$

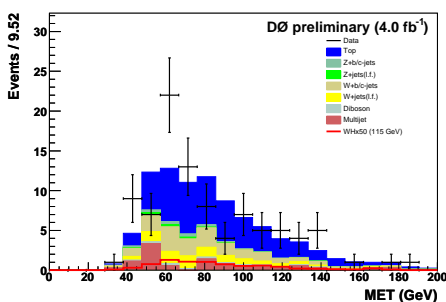

(n) $E_{\mathrm{T}}$

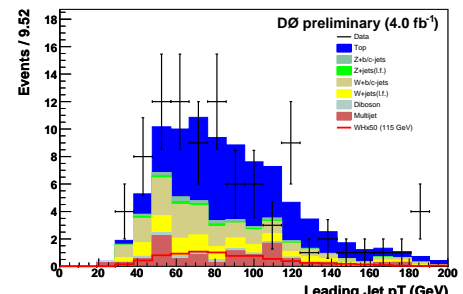

(c) $p_{T}($ jet 1$)$

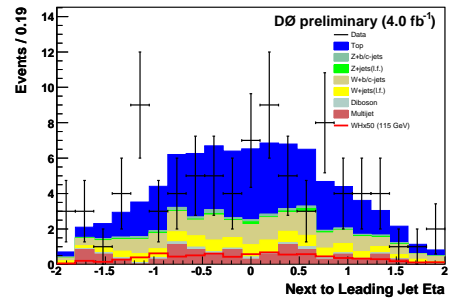

(f) $\eta_{d}($ jet 2$)$

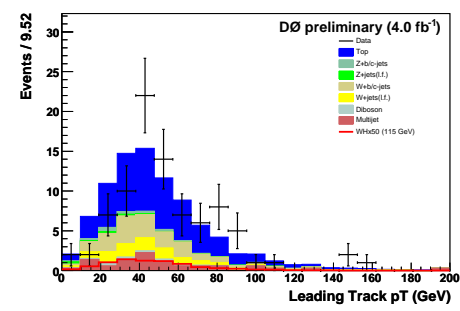

(i) $p_{T}(\operatorname{trk} 1)$

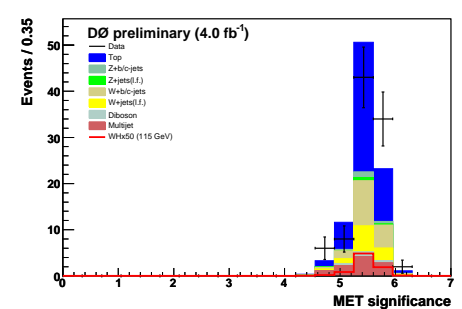

(1) $\ddot{E}_{\mathrm{T}}{ }_{\mathrm{SIC}}$

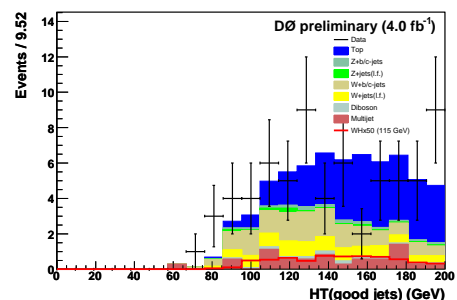

(o) $H_{T}$ (good jets)

Figure A.9: Data - Background comparisons in the analysis sample after one tight (VeryTight) b-tag selection for type 2 tau candidate events with 3 jets. 


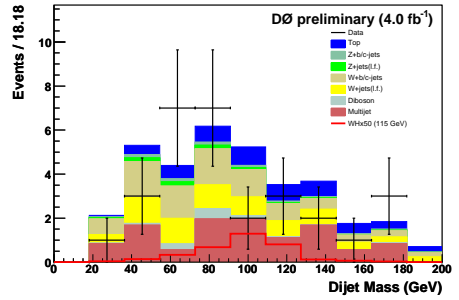

(a) Di-jet Mass

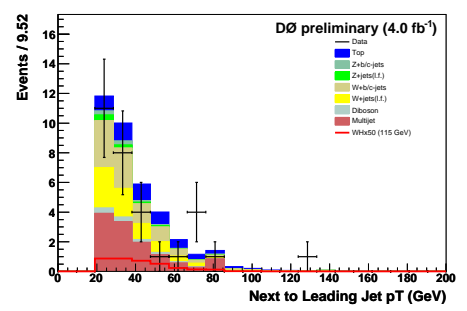

(d) $p_{T}(j e t 2)$

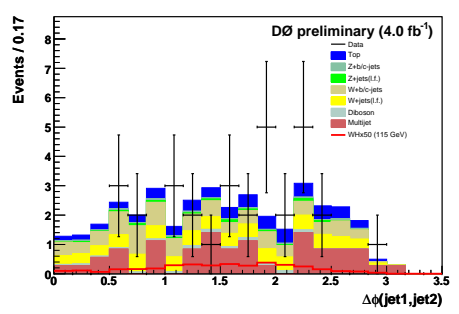

(g) $\Delta \phi($ jet1,jet2)

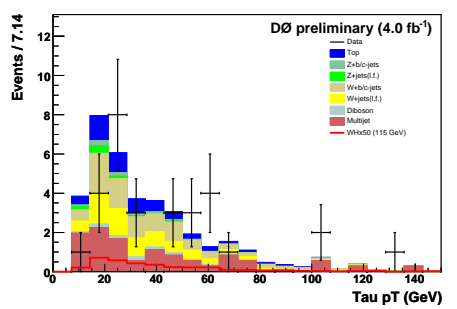

(j) $p_{T}(\tau)$

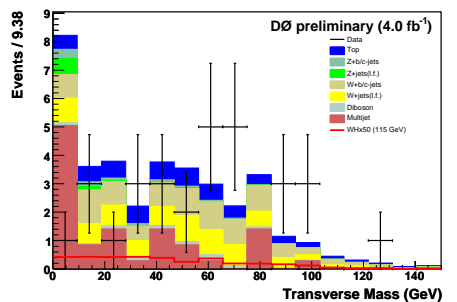

(m) $m_{T}(W)$

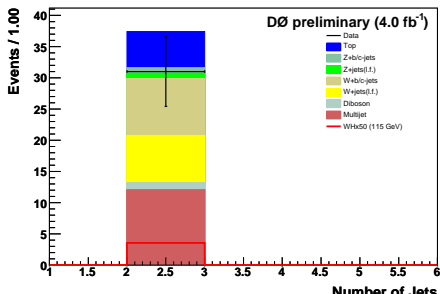

(b) Jet Multiplicity

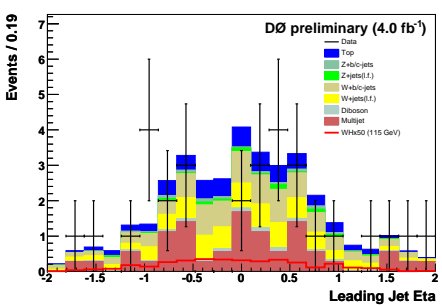

(e) $\eta_{d}(j e t 1)$

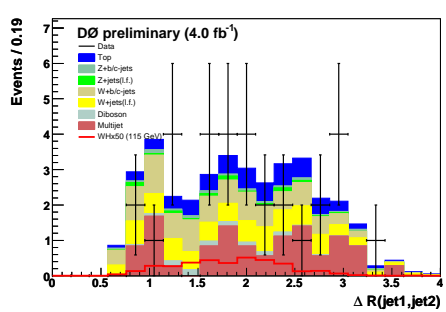

(h) $\Delta \mathrm{R}($ jet1,jet2)

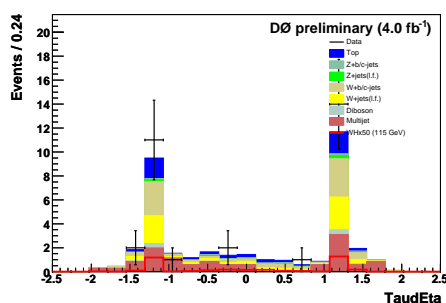

(k) $\eta_{d}(\tau)$

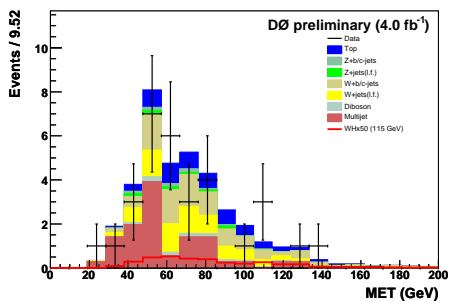

(n) $\ddot{E}_{\mathrm{T}}$

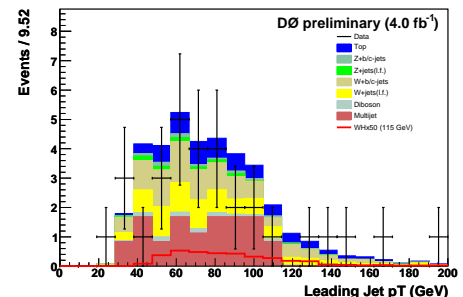

(c) $p_{T}(j e t 1)$

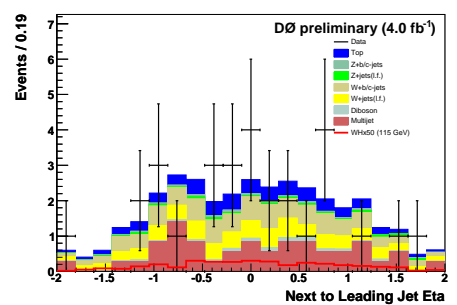

(f) $\eta_{d}(j e t 2)$

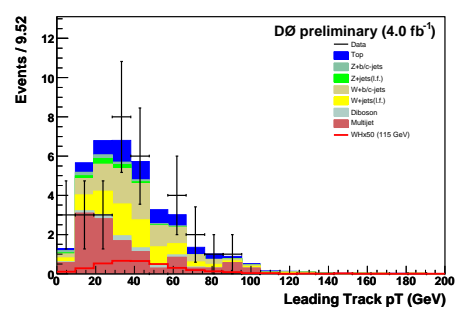

(i) $p_{T}(\operatorname{trk} 1)$

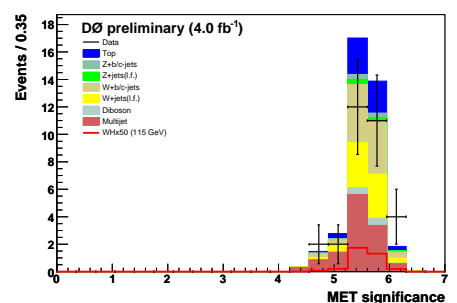

(1) $\not_{\mathrm{T}_{\mathrm{SIG}}}$

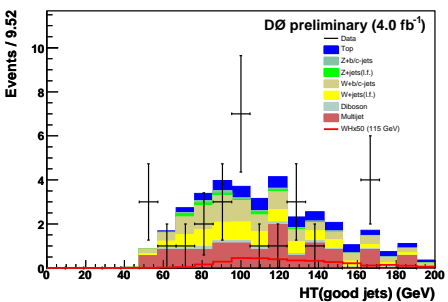

(o) $H_{T}$ (good jets)

Figure A.10: Data - Background comparisons in the analysis sample after one tight (VeryTight) b-tag selection for type 1 tau candidate events with 2 jets. 


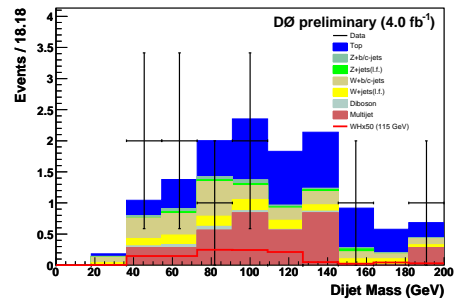

(a) Di-jet Mass

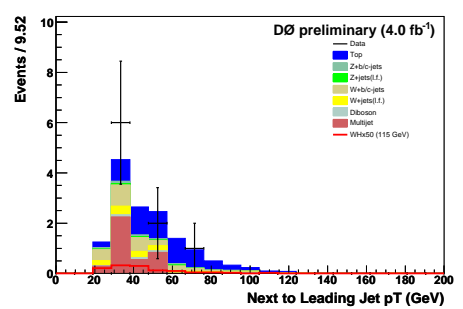

(d) $p_{T}(j e t 2)$

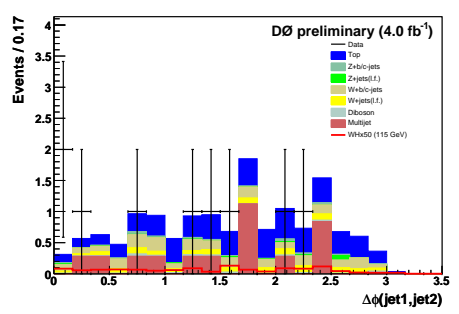

(g) $\Delta \phi($ jet1,jet2)

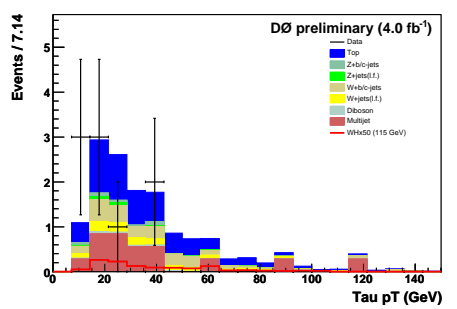

(j) $p_{T}(\tau)$

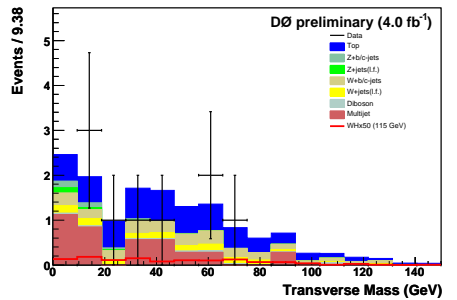

(m) $m_{T}(W)$

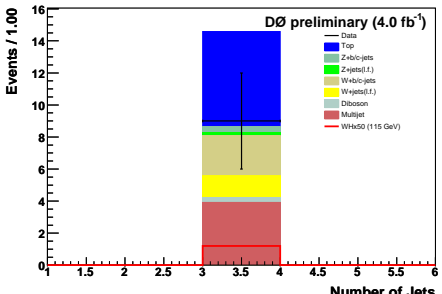

(b) Jet Multiplicity

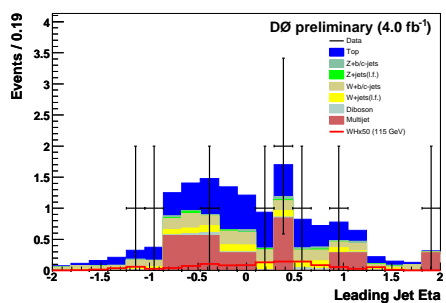

(e) $\eta_{d}(j e t 1)$

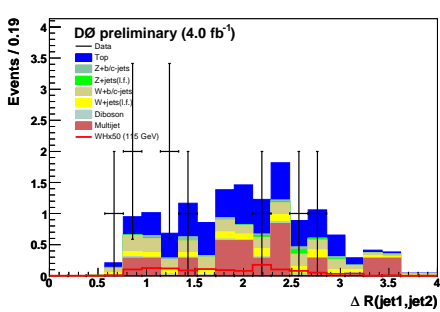

(h) $\Delta \mathrm{R}($ jet1,jet2)

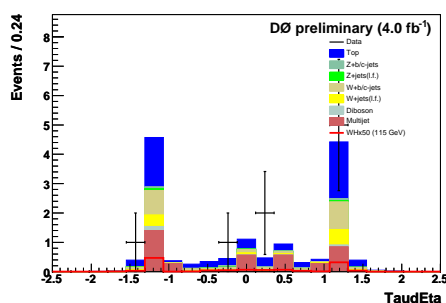

(k) $\eta_{d}(\tau)$

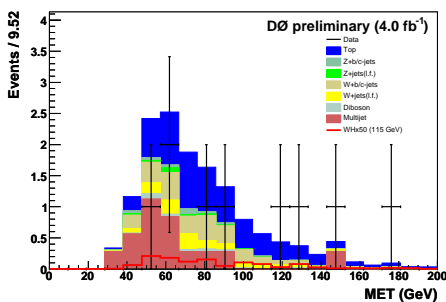

(n) $\ddot{E}_{\mathrm{T}}$

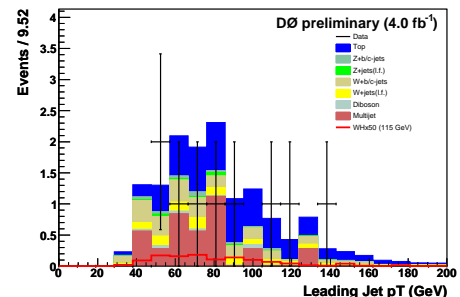

(c) $p_{T}(j e t 1)$

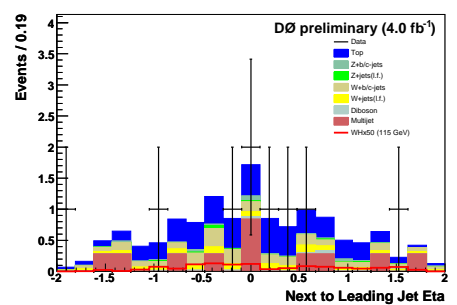

(f) $\eta_{d}(j e t 2)$

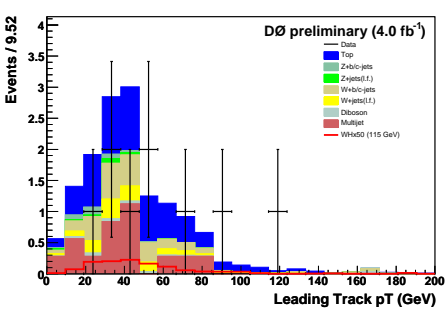

(i) $p_{T}(\operatorname{trk} 1)$

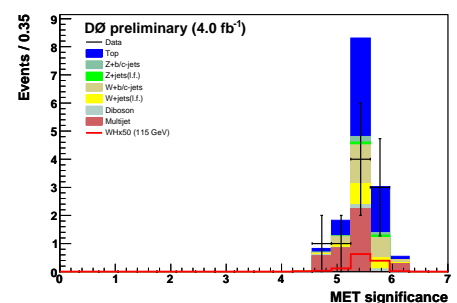

(1) $\not_{\mathrm{T}_{\mathrm{SIG}}}$

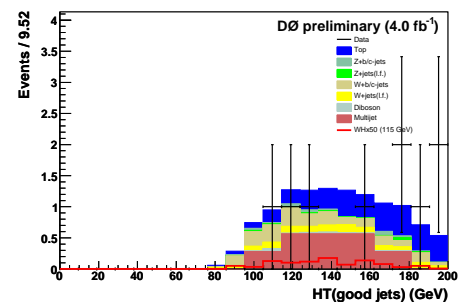

(o) $H_{T}$ (good jets)

Figure A.11: Data - Background comparisons in the analysis sample after one tight (VeryTight) b-tag selection for type 1 tau candidate events with 3 jets. 


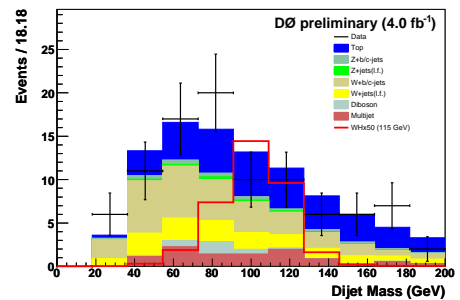

(a) Di-jet Mass

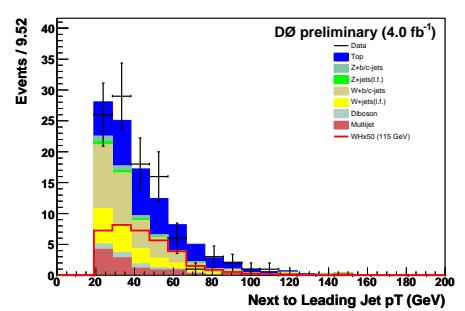

(d) $p_{T}($ jet 2$)$

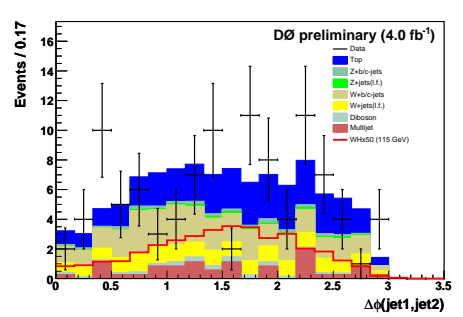

(g) $\Delta \phi($ jet1,jet2)

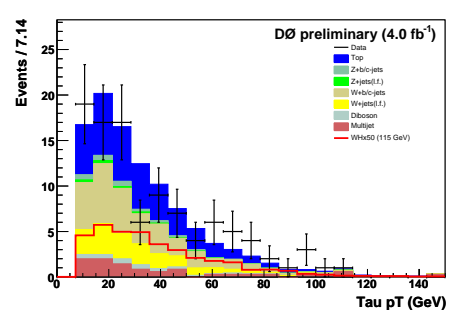

(j) $p_{T}(\tau)$

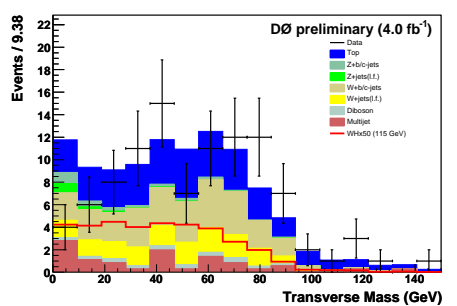

(m) $m_{T}(W)$

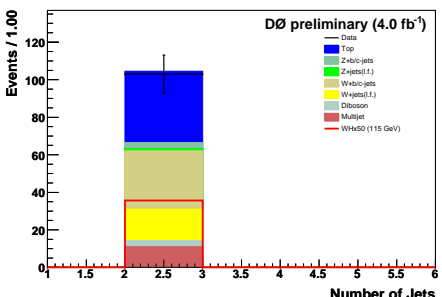

(b) Jet Multiplicity

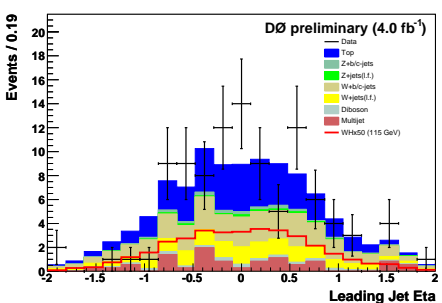

(e) $\eta_{d}(j e t 1)$

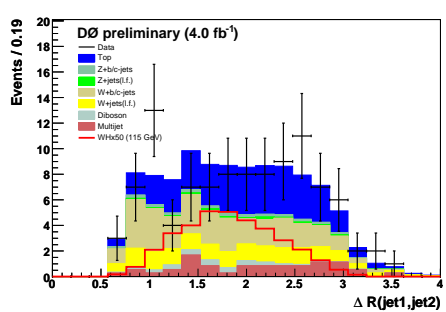

(h) $\Delta \mathrm{R}($ jet1,jet2)

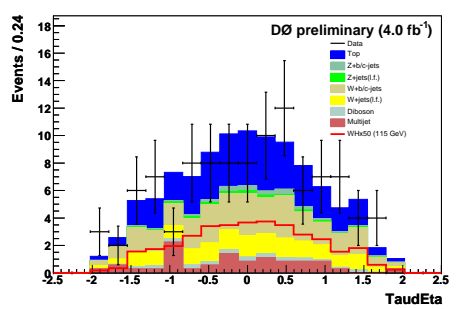

(k) $\eta_{d}(\tau)$

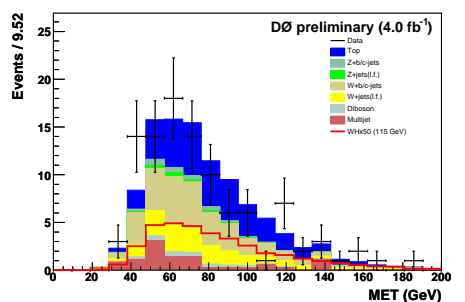

(n) $\ddot{E}_{\mathrm{T}}$

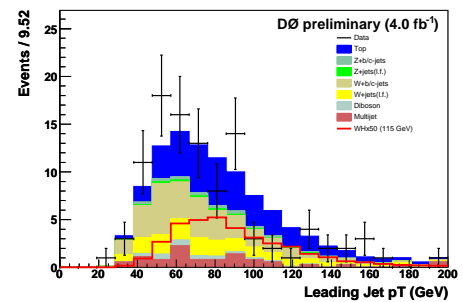

(c) $p_{T}($ jet 1$)$

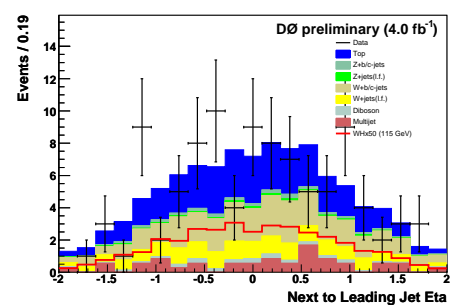

(f) $\eta_{d}(j e t 2)$

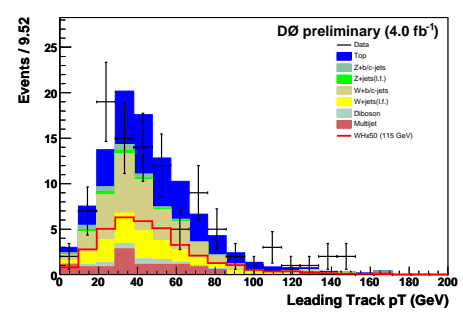

(i) $p_{T}(\operatorname{trk} 1)$

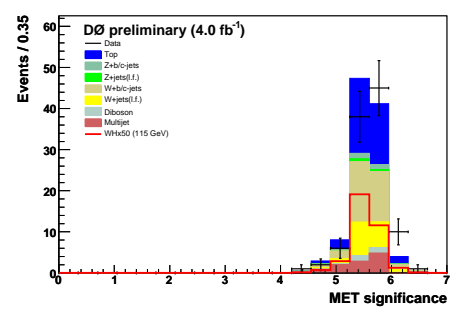

(l) $\not_{\mathrm{T}_{\mathrm{SIG}}}$

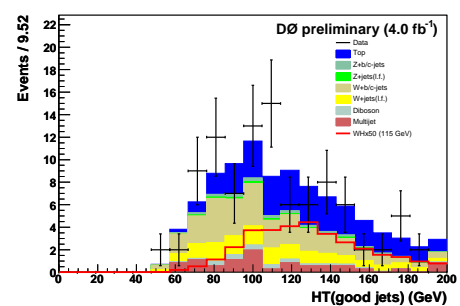

(o) $H_{T}($ good jets $)$

Figure A.12: Data - Background comparisons in the analysis sample after two loose (L3) b-tag selection for type 2 tau candidate events with 2 jets. 


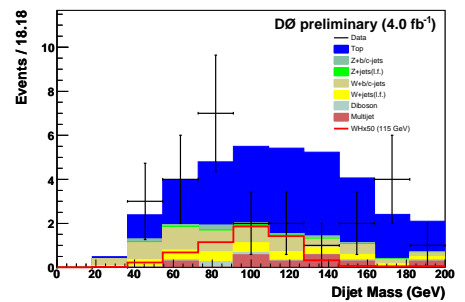

(a) Di-jet Mass

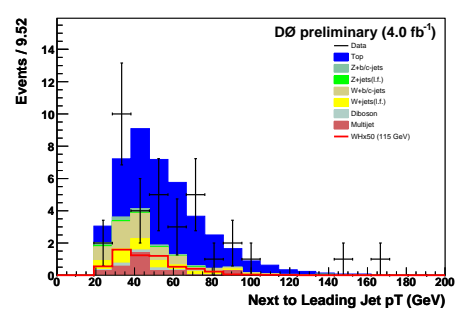

(d) $p_{T}($ jet 2$)$

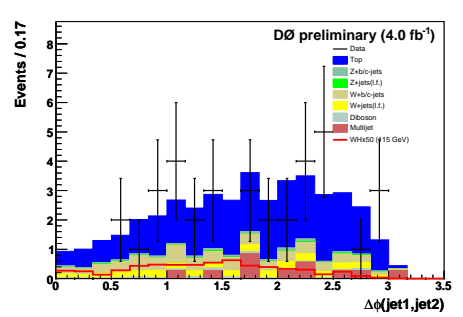

(g) $\Delta \phi($ jet1,jet2)

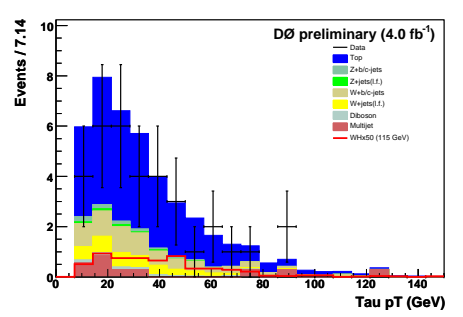

(j) $p_{T}(\tau)$

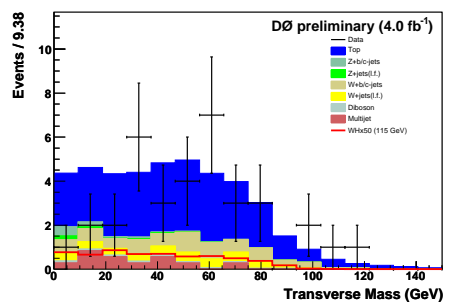

(m) $m_{T}(W)$

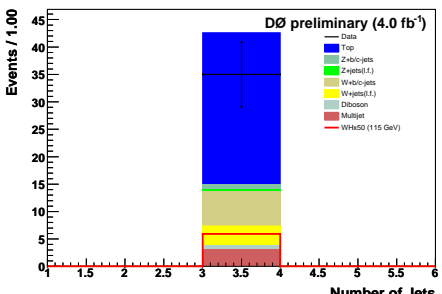

(b) Jet Multiplicity

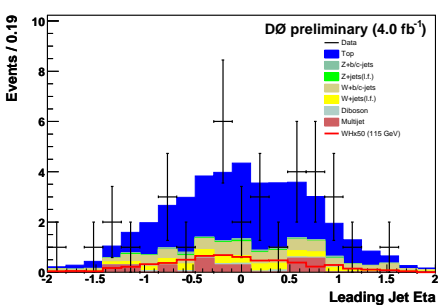

(e) $\eta_{d}(j e t 1)$

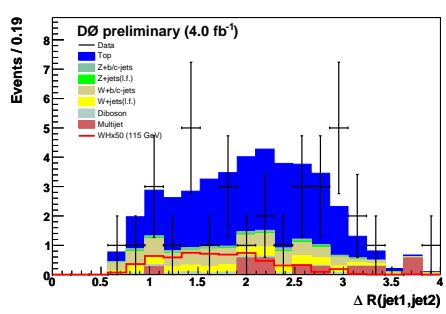

(h) $\Delta \mathrm{R}($ jet1,jet2)

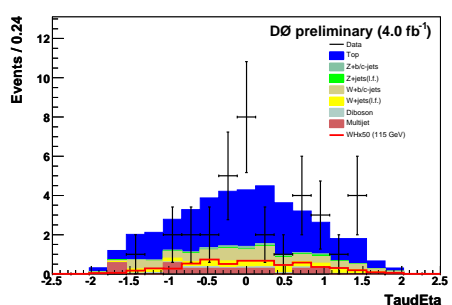

(k) $\eta_{d}(\tau)$

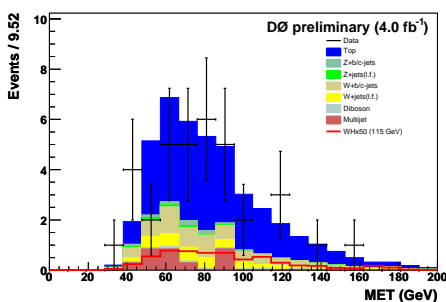

(n) $\ddot{E}_{\mathrm{T}}$

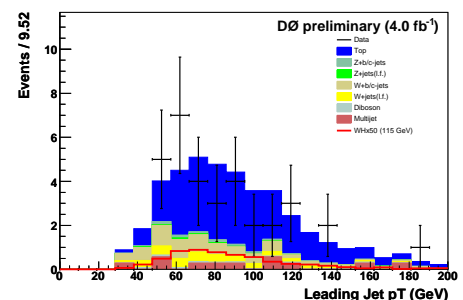

(c) $p_{T}($ jet 1$)$

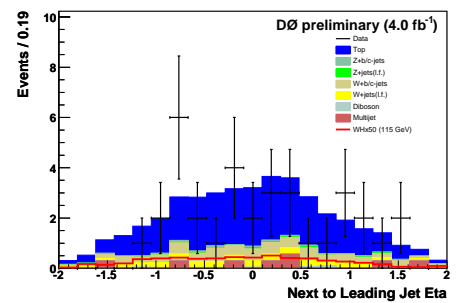

(f) $\eta_{d}(j e t 2)$

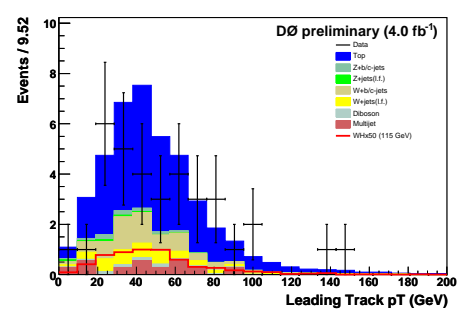

(i) $p_{T}(\operatorname{trk} 1)$

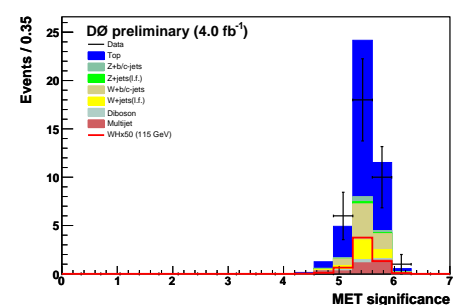

(l) $\not_{\mathrm{T}_{\mathrm{SIG}}}$

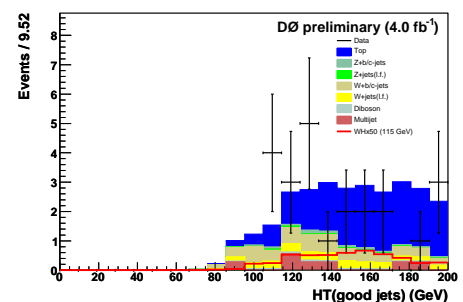

(o) $H_{T}($ good jets $)$

Figure A.13: Data - Background comparisons in the analysis sample after two loose (L3) b-tag selection for type 2 tau candidate events with 3 jets. 


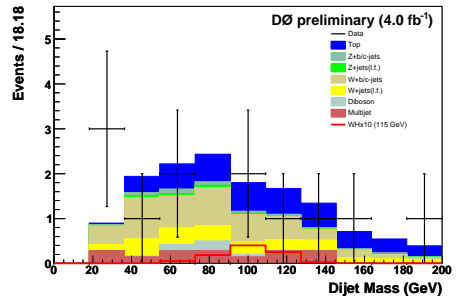

(a) Di-jet Mass

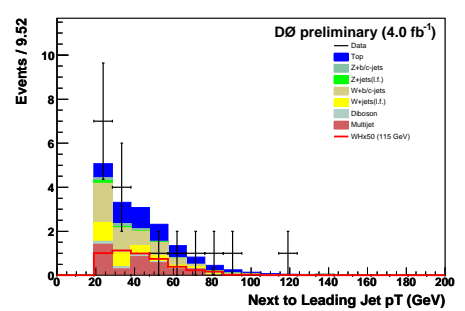

(d) $p_{T}($ jet 2$)$

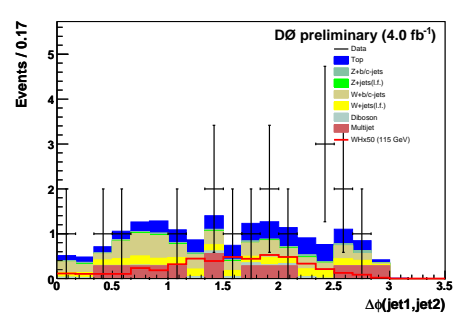

(g) $\Delta \phi($ jet1,jet2)

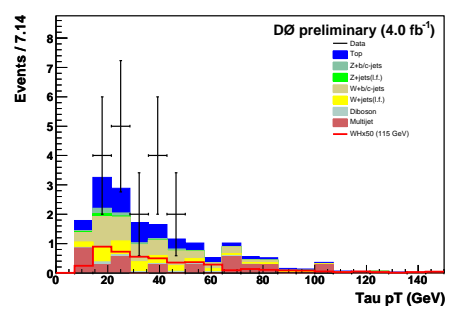

(j) $p_{T}(\tau)$

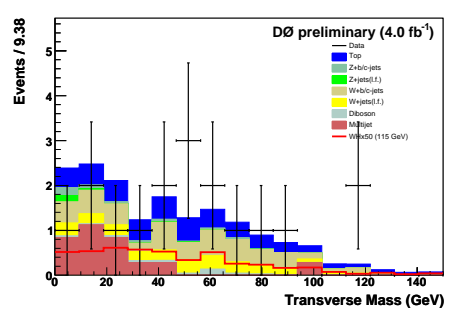

(m) $m_{T}(W)$

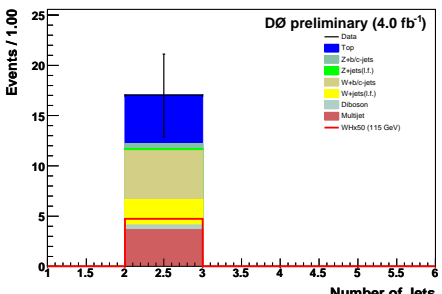

(b) Jet Multiplicity

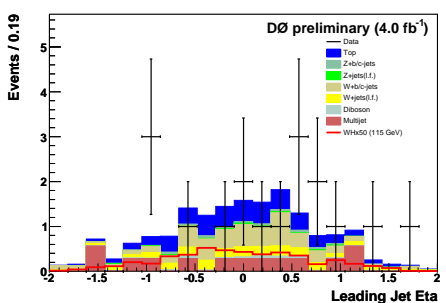

(e) $\eta_{d}(j e t 1)$

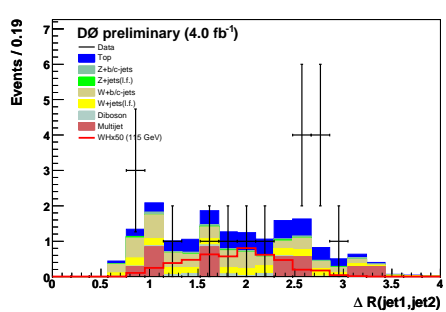

(h) $\Delta \mathrm{R}($ jet1,jet2)

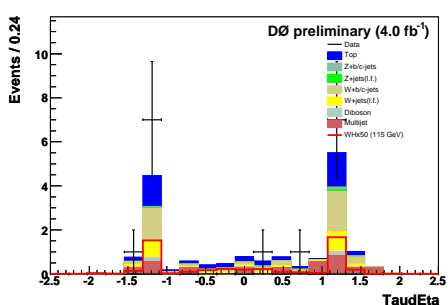

(k) $\eta_{d}(\tau)$

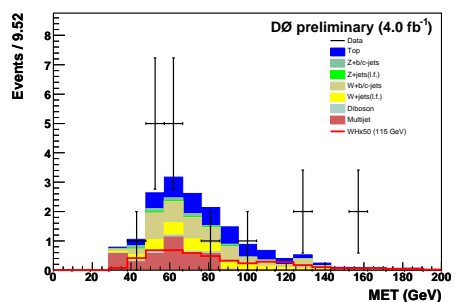

(n) $\ddot{E}_{\mathrm{T}}$

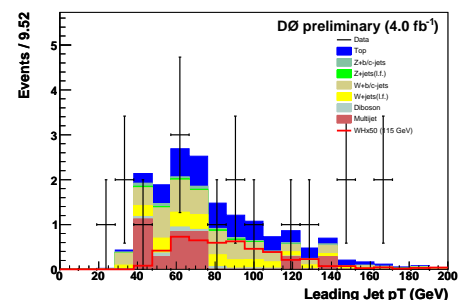

(c) $p_{T}($ jet 1$)$

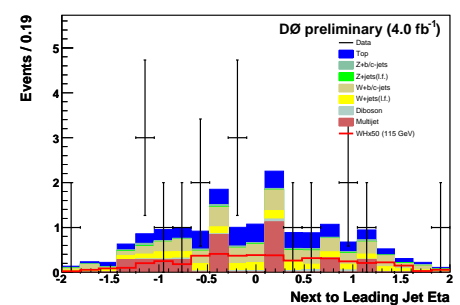

(f) $\eta_{d}(j e t 2)$

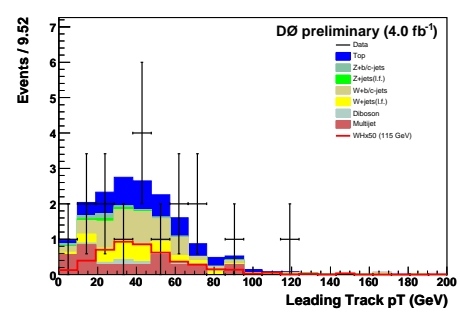

(i) $p_{T}(\operatorname{trk} 1)$

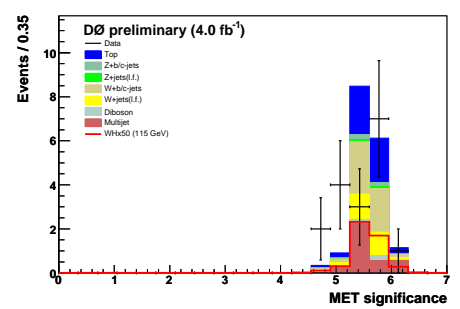

(1) $\ddot{E}_{\mathrm{T}_{\mathrm{SIC}}}$

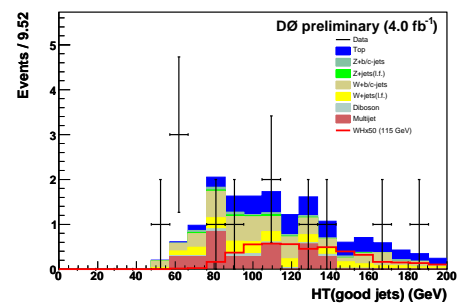

(o) $H_{T}($ good jets $)$

Figure A.14: Data - Background comparisons in the analysis sample after two loose (L3) b-tag selection for type 1 tau candidate events with 2 jets. 


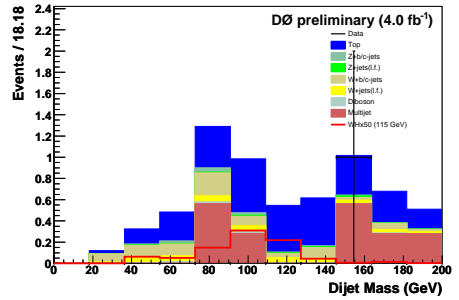

(a) Di-jet Mass

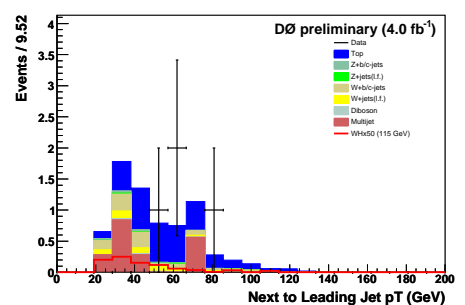

(d) $p_{T}($ jet 2$)$

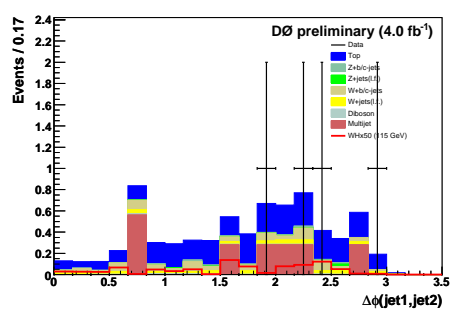

(g) $\Delta \phi($ jet1,jet2)

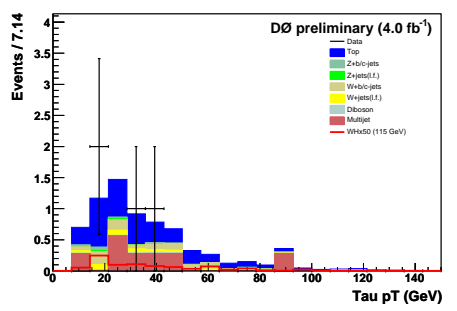

(j) $p_{T}(\tau)$

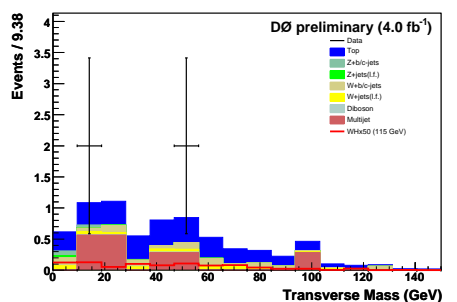

(m) $m_{T}(W)$

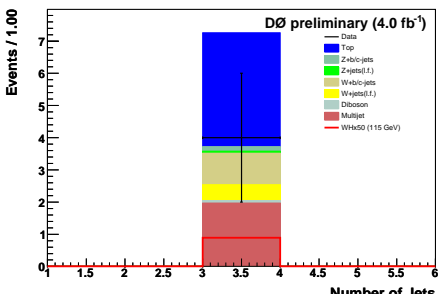

(b) Jet Multiplicity

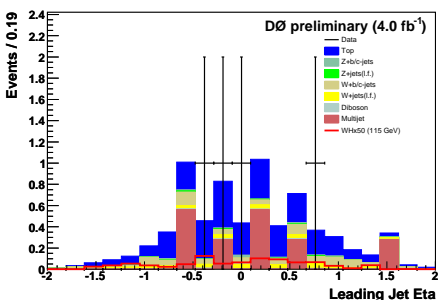

(e) $\eta_{d}(j e t 1)$

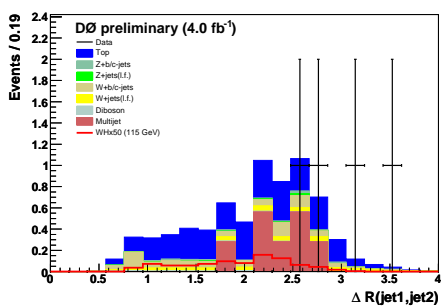

(h) $\Delta \mathrm{R}($ jet1,jet2)

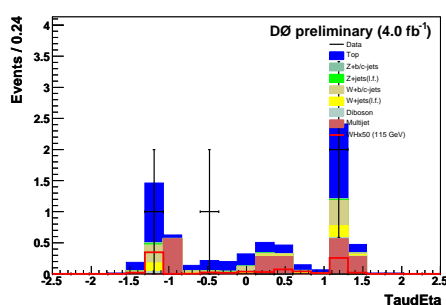

(k) $\eta_{d}(\tau)$

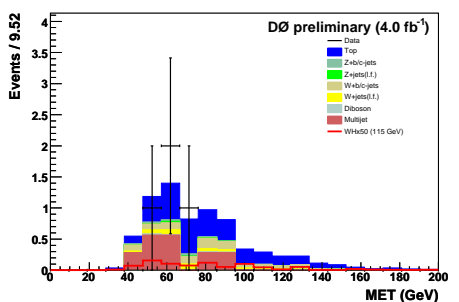

(n) $E_{\mathrm{T}}$

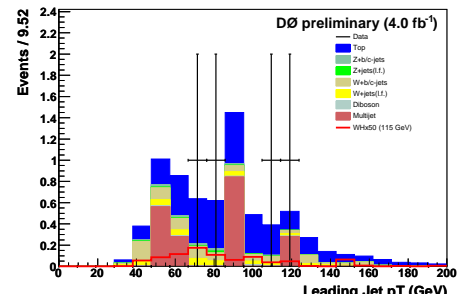

(c) $p_{T}(j e t 1)$

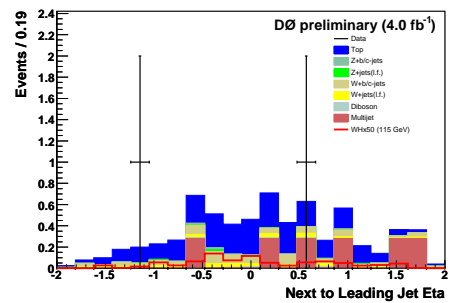

(f) $\eta_{d}(j e t 2)$

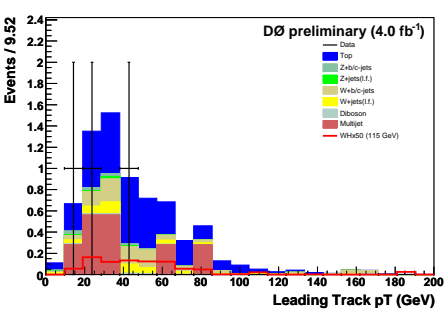

(i) $p_{T}(\operatorname{trk} 1)$

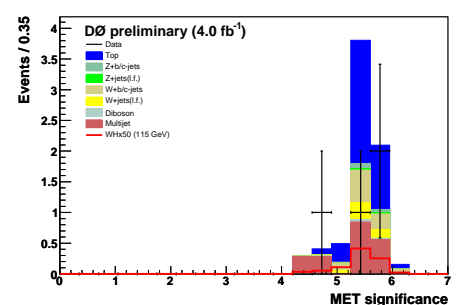

(1) $\not_{\mathrm{T}_{\mathrm{SIG}}}$

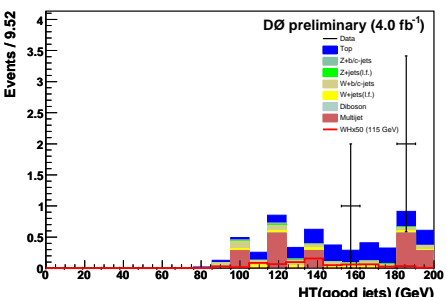

(o) $H_{T}$ (good jets)

Figure A.15: Data - Background comparisons in the analysis sample after two loose (L3) b-tag selection for type 1 tau candidate events with 3 jets. 


\section{References}

[1] J. Alcaraz et al., [ALEPH, DELPHI, L3 and OPAL Collaborations, LEP Electroweak Working Group], (2007), arxiv:0712.0929, updated for 2009 summer conferences, http://lepewwg.web.cern.ch/LEPEWWG/ .

[2] C. Amsler et al. (Particle Data Group), Phys. Lett. B667, 1 (2008) and 2009 partial update for the 2010 edition.

[3] Figure taken from: http://en.wikipedia.org/wiki/ File:Mecanismo_de_Higgs_PH.png

[4] T. Hahn, (2006), hep-ph/0607308.

[5] A. Djouadi, Comput. Phys. Commun. 108, 56 (1998), hep-ph/9704448.

[6] G. Abbiendi et al., [ALEPH, DELPHI, L3, OPAL and SLD Collaborations, LEP electroweak working group, SLD Electroweak and Heavy Flavour Groups], Phys. Rept. 427, 257 (2006).

[7] R. Barate et al., [ALEPH, DELPHI, L3 and OPAL Collaborations, LEP Working Group for Higgs Boson Searches], Phys. Lett. B565, 61 (2003).

[8] The TEVNPH Working Group, FERMILAB-PUB-09-060-E (2009).

[9] P. Rich. Nucl. Phys. A827 544-546 (2009). 
[10] V. M. Abazov et al., Phys. .Rev. Lett. 102, 251801 (2009).

[11] J. L. Rosner, J. Phys. G: Nucl. Part. Phys. 18 1575-1592 (1992).

[12] M. Artuso et al., PMC Physics A 33 (2009).

[13] J. Marriner, Nucl.Instrum.Meth. A532 11-18 (2004).

[14] J. Thompson, FERMILAB-TM 1909 (1994).

[15] V. M. Abazov et al., Nucl. Instrum. Meth. A565 463-537 (2006).

[16] J. Kim et al. Appl. Phys. Lett. 747 (1999).

[17] D. Smirnov, Nucl.Instrum.Meth. A598 94-97 (2008).

[18] S. Abachi et al., Nucl.Instrum.Meth. A324 53-76 (1993).

[19] DØ run coordination webpage. http://www-d0.fnal.gov/runcoor/

[20] O. Atramentov et al., DØ Note 5761 (2008).

[21] T. Gadfort et al., DØ Note 5157 (2006).

[22] D. Chakraborty et al., DØ Note 4210 (2003).

[23] S. Protopopescu and P. Svoisky, DØ Note 5094 (2006).

[24] V. M. Abazov et al., Phys. Lett. B670 292 (2009).

[25] V. M. Abazov et al., Phys. Lett. 101, 071804 (2008).

[26] J. L. Agram et al., DØ Note 4720 (2005).

[27] A. Schwartzman, DØ Note 4254 (2003).

[28] D. Bloch and B. Clément, DØ Note 4824 (2005). 
[29] D. Boline et al., DØ Note 4796 (2005).

[30] R. Demina et al., DØ Note 4049 (2002).

[31] T. Gadfort et al., DØ Note 5554 (2008).

[32] S. Lammers and G. Pawloski, DØ Note 4663 (2004).

[33] D. Stump et al., JHEP 10, 046 (2003).

[34] A. D. Martin et al., Phys. Lett. B604, 61 (2004).

[35] Y. Fisyak and J. Womersley, DØ Note 3191 (1997).

[36] T. Sjostrand, et al., Comput. Phys. Commun. 135238 (2001).

[37] M. L. Mangano et al., JHEP 07, 001 (2003).

[38] E. E. Boos et al., (1994), hep-ph/9503280.

[39] T. Nunneman, http://www-cluedo.fnal.gov/ nunne/ cross-sections/mcfm_cross-sections.html.

[40] S. Muanza, DØ Note 5300 (2006).

[41] M. Sanders, http://www-d0.fnal.gov/ msanders/Higgs/CSTimesBr.html

[42] T. Nunneman, DØ Note 4476 (2004).

[43] F. Couderc, http://www-d0.hef .kun.nl//askArchive.php? base=agenda\&categ=a09917\&id=a09917s1t1/transparencies (2009).

[44] N. Makovec and J-F. Grivaz, DØ Note 4914 (2005).

[45] M. Shamim and T. Bolton, DØ Note 5565 (2008).

[46] G. Hesketh, DØ Note 5786 (2008). 
[47] M. Rangel, http://www-d0.hef .kun.nl//askArchive.php? base=agenda\&categ=a09320\&id=a09320s1t1/transparencies (2009).

[48] S. Calvet, DØ Note 5944 (2009).

[49] S. Calvet et al., DØ Note 5857 (2009).

[50] K. Peters et al., DØ Note 5669 (2008).

[51] http://www-d0.fnal.gov/Run2Physics/top/public/fall06/singletop /DT_public_webpage/DecisionTrees.html

[52] B. P. Roe et al., Nucl.Instrum.Meth. A543 577-584 (2005).

[53] S. Frixione, B. R. Webber, hep-ph/0204244 (2002).

[54] N. Kidonakis, Phys. Rev. D. 74114012 (2006).

[55] S. Moch, P. Uwer, arXiv:0807.2794.

[56] G. Davies et al., General Higgs Meeting News 090709, http://www-d0.hef .kun.nl///fullAgenda.php?ida=a091096\#2009-07-09.

[57] T. Andeen et al., DØ Note 5398 (2007).

[58] W. Fisher, http://www-cluedo.fnal.gov/ wfisher/d0_private /AlpDocs/alpgenSysts.h.

[59] The DØ Collaboration, DØ Note 5972-CONF (2009).

[60] A. Read, J. Phys. G: Nucl. Part. Phys. 28 2693, (2002).

[61] T. Junk, Nucl. Instrum. Meth A434 435, (1999).

[62] W. Fisher, DØ Note 4975, (2006).

[63] W. Fisher, DØ Note 5309, (2006). 
[64] W. Fisher, DØ Note 5595, (2008).

[65] The DØ Collaboration, DØ Note 5984-CONF (2009).

[66] The CDF Collaboration, CDF Note 9674 (2009).

[67] ATLAS Collaboration, Expected Performance of the ATLAS Experiment, Detector, Trigger and Physics, CERN-OPEN-2008-020, Geneva, 2008.

[68] The ATLAS Experiment. http://atlas.ch.

[69] The Large Hadron Collider. http://lhc.web.cern.ch/lhc/.

[70] H. Burkhardt for the LHC team.

"Status of the LHC and commissioning plans", Talk at Lepton-Photon 2009. http://indico.desy.de/conferenceOtherViews.py? view=standard\&conf $\mathrm{Id}=1761$. 